\title{
Modeling anthracycline-induced cardiotoxicity with patient-specific iPSCs
}

\author{
Dissertation \\ for the award of the degree \\ “Doctor rerum naturalium” (Dr. rer. nat.) \\ of the Georg-August-Universität Göttingen \\ within the doctoral program Biology \\ of the Georg-August University School of Science (GAUSS)
}

submitted by

Luis Peter Haupt

from Braunschweig

Göttingen, 2018 
Thesis Committee

Prof. Dr. rer. nat. Sigrid Hoyer-Fender, Department of Developmental Biology, JohannFriedrich-Blumenbach Institute of Zoology and Anthropology Göttingen

Prof. Dr. rer. nat. Susanne Lutz, Institute of Pharmacology and Toxicology, University Medical Center Göttingen

Dr. rer. nat. Katrin Streckfuß-Bömeke, Department of Cardiology and Pneumology, University Medical Center Göttingen

Members of the Examination Board:

Reviewer: Prof. Dr. rer. nat. Sigrid Hoyer-Fender, Department of Developmental Biology, Johann-Friedrich-Blumenbach Institute of Zoology and Anthropology Göttingen

Second Reviewer: Prof. Dr. rer. nat. Susanne Lutz, Institute of Pharmacology and Toxicology, University Medical Center Göttingen

Further members of the Examination Board:

Dr. rer. nat. Katrin Streckfuß-Bömeke, Department of Cardiology and Pneumology, University Medical Center Göttingen

Prof. Dr. med. Dörthe Katschinski, Department of Cardiovascular Physiology, University Medical Center Göttingen

Prof. Dr. rer. nat. Ernst A. Wimmer, Department of Developmental Biology, Johann-FriedrichBlumenbach Institute of Zoology and Anthropology Göttingen

Prof. Dr. rer. nat. Rüdiger Behr, German Primate Center, Leibniz Institute for Primate Research

Date of the oral examination: February $20^{\text {th }}, 2018$ 


\section{Table of contents}

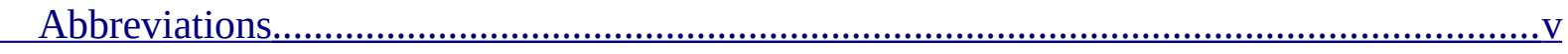

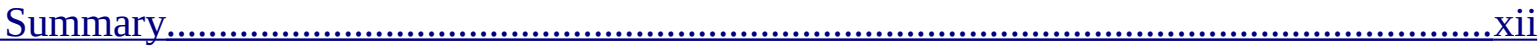

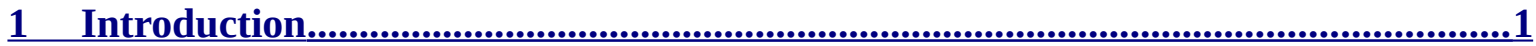

1.1 Anthracycline-induced cardiotoxicity ...........................................................

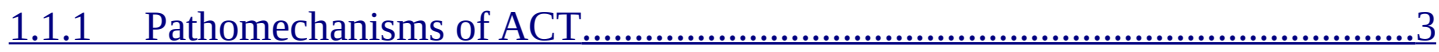

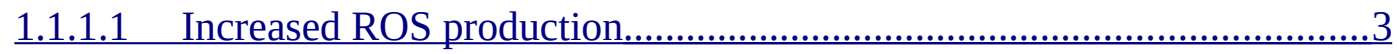

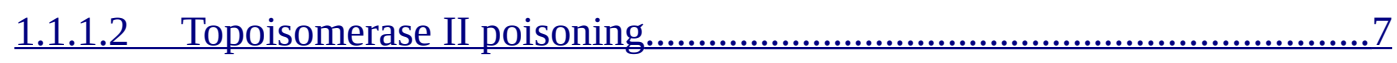

1.1.1.3 Disturbance of Calcium signaling............................................

1.1.1.4 Disarray of sarcomeric cytoskeleton, NRG1/ErbB2 signaling and the

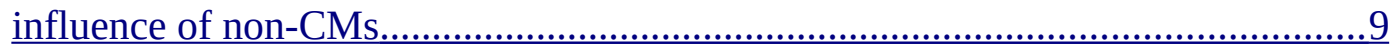

1.1.2 Treatment and prophylactic therapy of ACT .........................................10

1.1.3 Pharmacogenetics of ACT................................................................ 11

1.2 Induced pluripotent stem cells in cardiovascular medicine..............................13

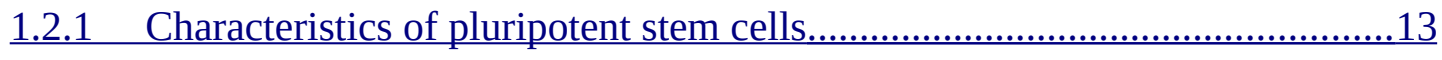

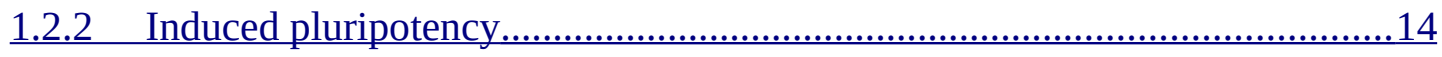

1.2.3 Cardiac differentiation of hiPSCs...................................................... 17

1.2.4 Cardiac applications of hiPSCs.........................................................19

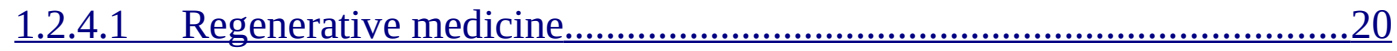

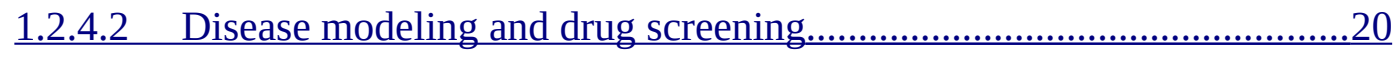

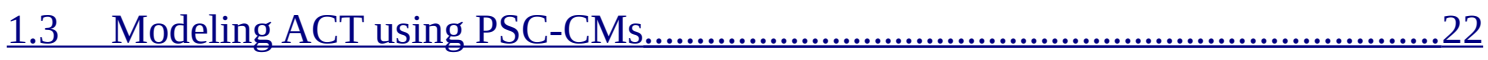

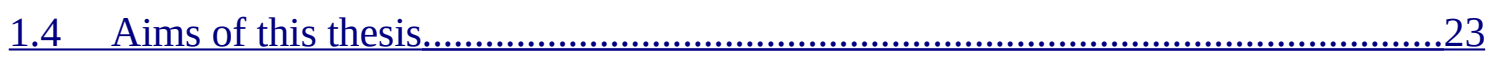

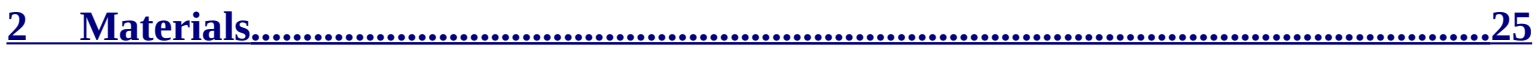

2.1 Chemicals, reagents, basal media and enzymes............................................25

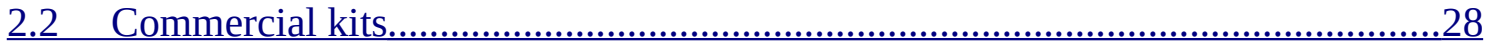

$\underline{2.3}$ Buffers and solutions for molecular biological analyses...............................28

2.4 Media supplements, factors and solutions for cell culture.................................32

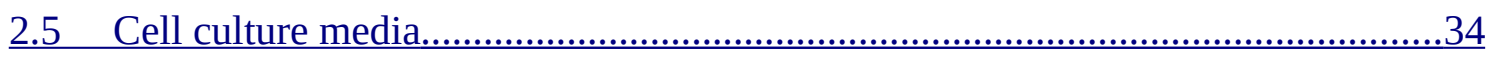

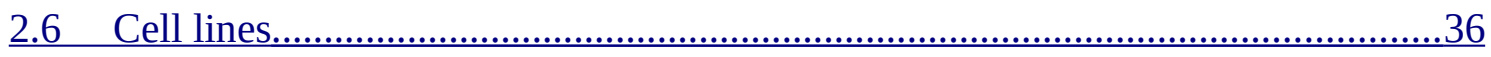

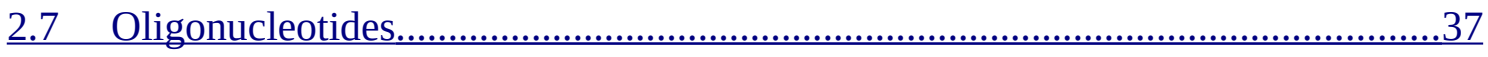

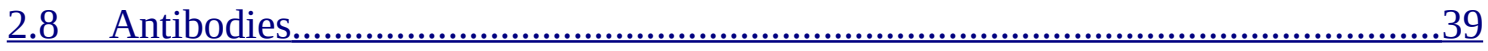


2.8.1 Primary antibodies........................................................................................39

2.8.2 Secondary antibodies and fluorophore-conjugated probes............................41

2.9 DNA plasmids.............................................................................................41

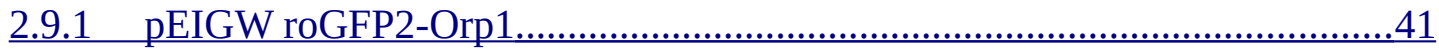

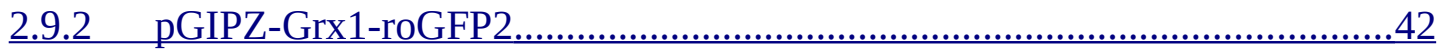

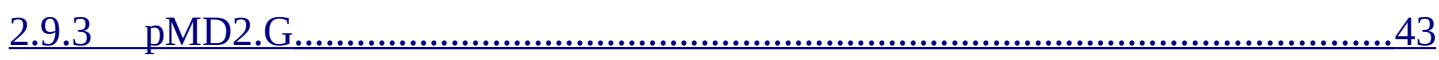

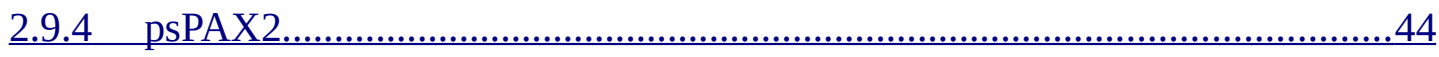

2.10 Disposable items.....................................................................................45

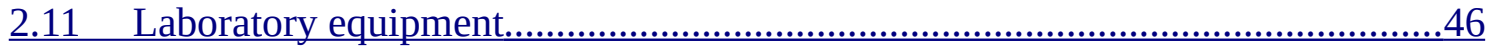

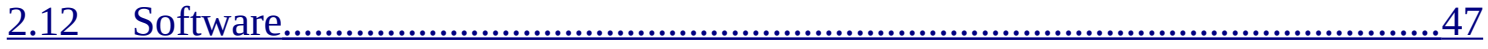

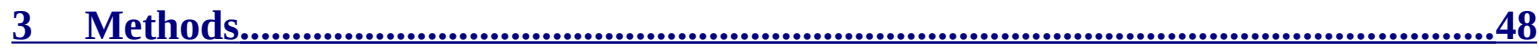

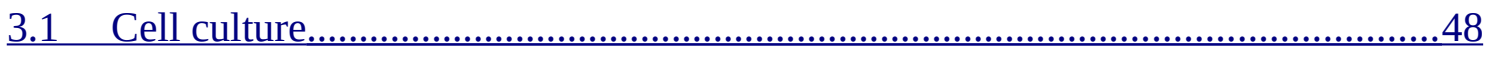

3.1.1 Isolation and cultivation of human primary dermal fibroblasts......................48

3.1.2 Isolation and cultivation of mouse embryonic fibroblasts..............................48

3.1.3 Generation of hiPSC lines.........................................................................48

3.1.4 Cultivation of hiPSCs on MEFs or geltrex...…………………………….....49

3.1.5 Freezing of dermal fibroblasts and iPSCs on MEFs and geltrex...................49

3.1.6 Thawing of HEK cells and iPSCs on MEFs or geltrex................................50

3.1.7 Spontaneous in vitro differentiation........................................................50

3.1.8 Cardiac differentiation of hiPSCs and metabolic selection............................51

3.1.9 Preparation and preservation of cell samples for analyses.............................51

3.1.10 Cultivation of HEK-293T cells...........................................................51

3.1.11 Lentivirus production...............................................................................52

3.1.12 Lentiviral transduction of iPSC-CMs.......................................................52

3.1.13 Engineered heart muscle ……………………………………………….....52

3.2 Molecular biology techniques.................................................................55

3.2.1 Isolation of genomic DNA and sequencing of SNPs...................................53

3.2.2 Alkaline phosphatase staining.......................................................................53

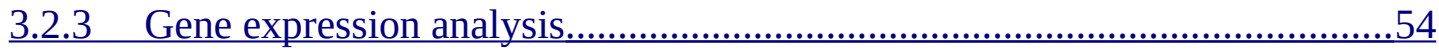

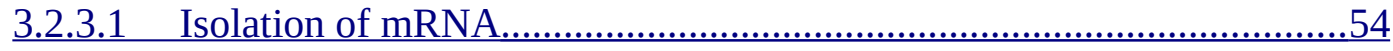

3.2.3.2 Reverse transcription...........................................................................54

3.2.3.3 Polymerase chain reaction (PCR) analysis.............................................55 
3.2.3.4 Quantitative real-time polymerase chain reaction (qRT-PCR) analysis.56

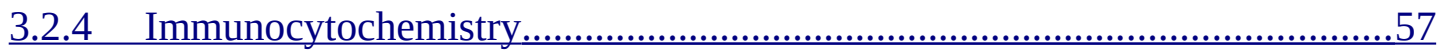

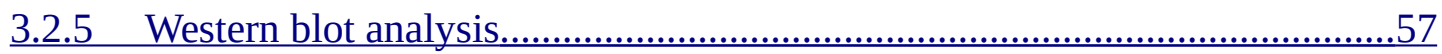

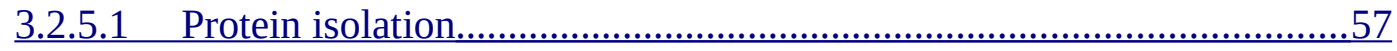

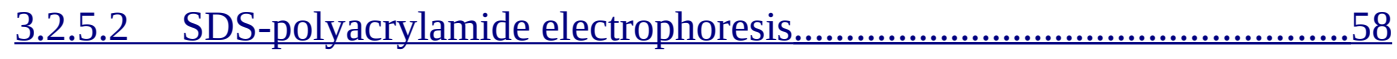

3.2.5.3 Protein transfer and detection...............................................58

3.2.6 Amplex Red Hydrogen Peroxide Assay.....................................................58

3.2.7 Plasmid isolation from E. coli culture...................................................59

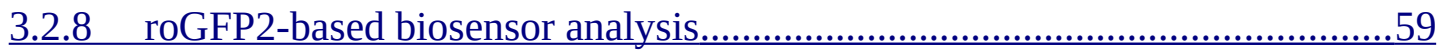

3.2.9 Annexin $\mathrm{V}$ affinity assay and flow cytometry..........................................60

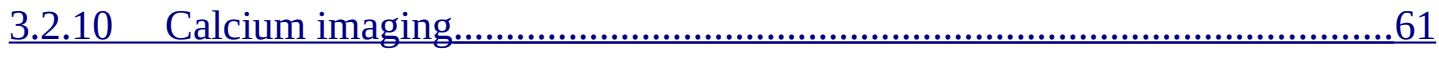

3.3 In vivo teratoma formation and histological analysis....................................61

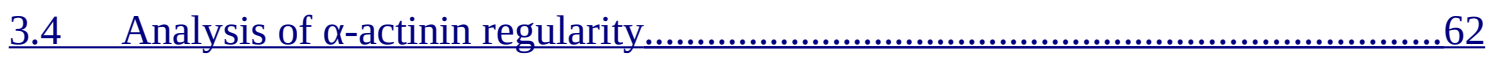

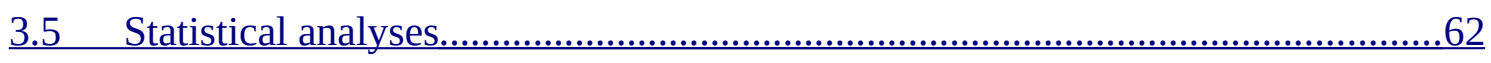

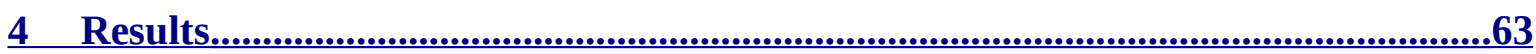

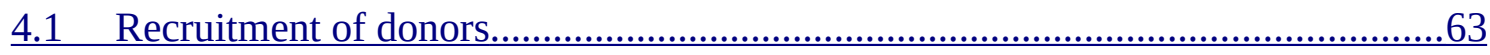

4.2 Generation and characterization of hiPSCs.................................................66

4.2.1 Generation of hiPSCs..................................................................66

4.2.2 Expression of pluripotency-related genes................................................66

4.2.3 Differentiation potential in vitro and in vivo.........................................68

$\underline{4.3}$ Generation of iPSC-derived cardiomyocytes........................................ 72

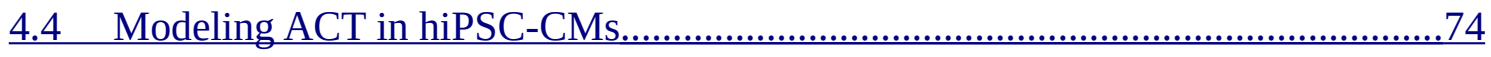

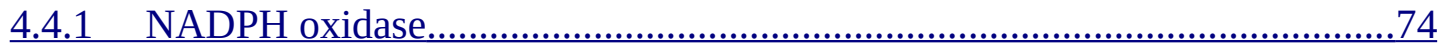

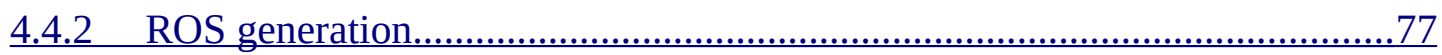

4.4.2.1 Amplex Red Hydrogen Peroxide Assay...........................................77

4.4.2.2 roGFP2-based biosensor analyses..........................................

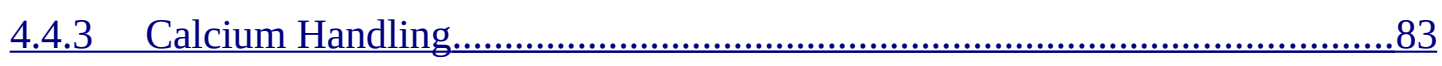

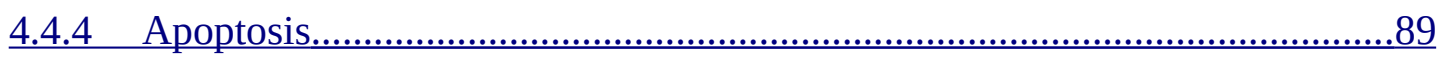

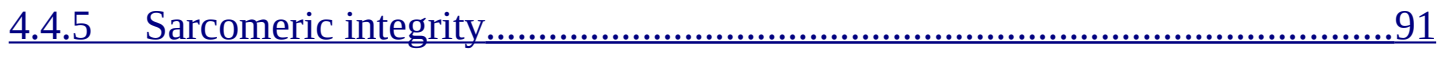

4.4.6 Mechanical functionality...........................................................93

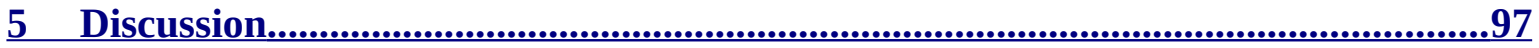

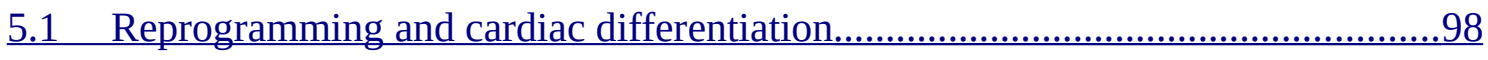


5.1.1 Generation of hiPSCs from ACT patients and controls............................98

5.1.2 Generation of hiPSC-CMs from ACT patients and controls......................99

5.2 Modeling ACT with hiPSC-CMs from ACT patients and controls....................100

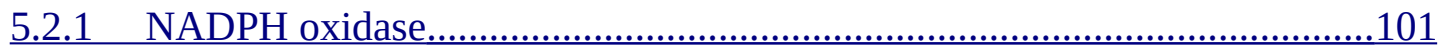

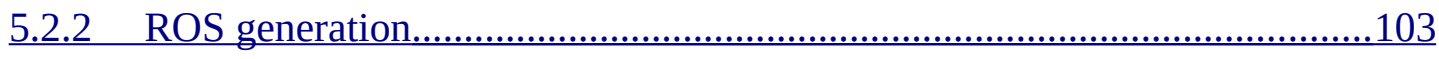

5.2.2.1 Alternative approaches for ROS analysis.................................108

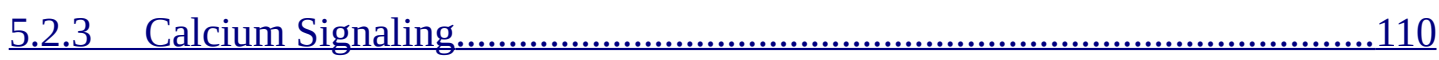

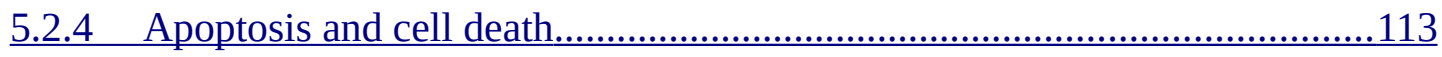

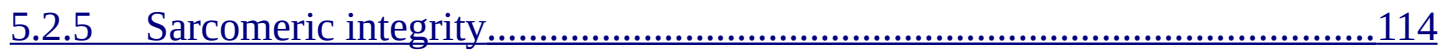

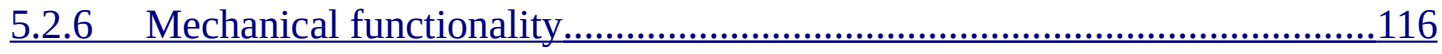

5.2.7 Screening for protectants against ACT using the iPSC-CM model.............118

5.2.8 Limitations of this work..................................................................119

5.2.9 Conclusions and proposed model................................................120

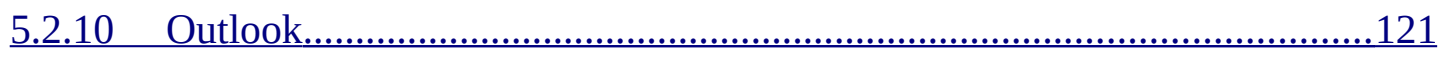

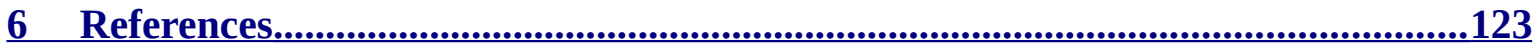

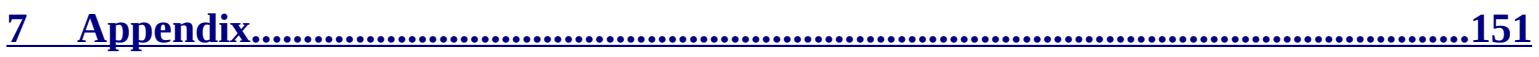

Acknowledgments....................................................................................................

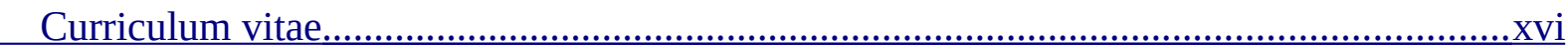

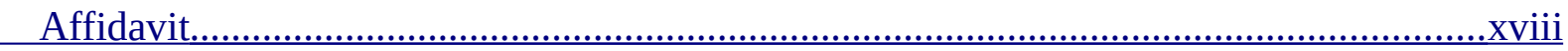




\section{Abbreviations}

$\cdot \mathrm{O}_{2}{ }^{-}$

$\cdot \mathrm{OH}$

${ }^{\circ} \mathrm{C}$

A

ABCC

ACT

AFP

ALB

ANOVA

APS

ATP

BMP

bp

BSA

C

c-myc

$\mathrm{Ca}^{2+}$

$\mathrm{CaCl}_{2}$

$\mathrm{CaM}$

CaMKII

CamKII

cAMP

Cas9

CASQ2

cDNA

$\mathrm{CHF}$

CIU

$\mathrm{CM}$

$\mathrm{CO}_{2}$

CPVT

CRISPR

CSA
Superoxide radical

Hydroxyl radical

Degree Celsius

Adenine

ATP Binding Cassettes Subfamily C Member 2

Anthracycline-induced cardiotoxicity

Alpha fetoprotein

Albumin

Analysis of variance

Ammonium persulfate

Adenosine triphosphate

Bone morphogenic protein

Base pair

Bovine serum albumin

Cytosine

V-myc myelocytomatosis viral oncogene homolog

Calcium ion

Calcium chloride

Calmodulin

$\mathrm{Ca}^{2+} /$ calmodulin-dependent protein kinase II

Ca2+/calmodulin-dependent protein kinase II

Cyclic adenosine monophosphate

CRISPR-associated-9

Calsequestrin 2

Complementary DNA

Congestive heart failure

Collective infectious unit

Cardiomyocyte

Carbon dioxide

Catecholaminergic polymorphic ventricular tachycardia

Clustered Regularly Interspaced Short Palindromic Repeats

Cross-sectional area 


\begin{tabular}{|c|c|}
\hline CT & Cycle threshold \\
\hline cTNT & Cardiac troponin $\mathrm{T}$ \\
\hline Ctrl & Control \\
\hline Cy & Amplification cycles of PCR \\
\hline CYBA & Cytochrome B-245 alpha chain \\
\hline $\mathrm{d}$ & Day \\
\hline DAPI & 4',6-Diamidino-2-phenylindole \\
\hline DEX & Dexrazoxane \\
\hline DMEM & Dulbecco's modified eagle medium \\
\hline DMSO & Dimethyl sulfoxide \\
\hline DNA & Deoxyribonucleic acid \\
\hline dNTPs & Deoxyribonucleotide \\
\hline DOX & Doxorubicin \\
\hline DPBS & Dulbecco's phosphate buffered saline \\
\hline $\mathrm{DR} 4 / 5$ & Death receptor $4 / 5$ \\
\hline DTT & Dithiothreitol \\
\hline $\mathrm{E}$ & Redoxpotential \\
\hline E8 & Essential 8 \\
\hline EBs & Embryoid bodies \\
\hline ECCs & Embryonic carcinoma cells \\
\hline EDTA & Ethylenediaminetetra-acetic acid \\
\hline EGCs & Embryonic germ cells \\
\hline EHM & Engineered heart muscle \\
\hline EpiSCs & Epiblast-derived stem cells \\
\hline ErbB2/4 & Epidermal growth factor receptor \\
\hline ESCs & Embryonic stem cells \\
\hline FBS & Fetal bovine serum \\
\hline FDA & United States Food and Drug Administration \\
\hline $\mathrm{Fe}$ & Iron \\
\hline FGF & Fibroblast growth factor \\
\hline FGF2 & Fibroblast growth factor-2 \\
\hline For & Forward primer \\
\hline Fp & Flavoprotein \\
\hline FS & Fractional shortening \\
\hline
\end{tabular}




\begin{tabular}{|c|c|}
\hline g & Gram \\
\hline G & Guanine \\
\hline$g$ & Gravitational force \\
\hline GAPDH & Glyceraldehyde-3-phosphate dehydrogenase \\
\hline Gata4 & GATA binding protein 4 \\
\hline GDF3 & Growth differentiation factor-3 \\
\hline GFP & Green fluorescent protein \\
\hline Grx1 & glutaredoxin-1 \\
\hline GSH & Reduced glutathione \\
\hline GSK3 & Glycogen synthase kinase 3 \\
\hline GSSG & Oxidized glutathione \\
\hline h & Hour \\
\hline $\mathrm{H}+\mathrm{E}$ & Haematoxylin eosin staining \\
\hline $\mathrm{H}_{2} \mathrm{O}$ & Water \\
\hline $\mathrm{H}_{2} \mathrm{O}_{2}$ & Hydrogen peroxide \\
\hline hbFGF & Human basic fibroblast growth factor \\
\hline HEPES & 4-(2-hydroxyethyl)-1-piperazineethanesulfonic acid \\
\hline hES-medium & Human embryonic stem cell medium \\
\hline Hey2 & Hairy/enhancer-of-split related with YRPW motif protein 2 \\
\hline HFB-medium & Human fibroblast medium \\
\hline HPRT & Hypoxanthine-guanine phosphoribosyltransferase \\
\hline IgG & Immunoglobulin G \\
\hline $\operatorname{IgM}$ & Immunoglobulin $\mathrm{M}$ \\
\hline IMDM & Iscove's modified Dulbecco's medium \\
\hline iPSC-CMs & Induced pluripotent stem cell-derived cardiomyocytes \\
\hline iPSCs & Induced pluripotent stem cells \\
\hline ISO & Isoprenaline hydrochloride \\
\hline $\mathrm{kb}$ & Kilobase \\
\hline $\mathrm{KCl}$ & Potassium chloride \\
\hline KLF4 & Krüppel-like factor \\
\hline $\mathrm{L}$ & Liter \\
\hline LIF & Leukemia inhibitory factor \\
\hline LIN28 & Cell lineage abnormal 28 \\
\hline LTCC & L-type calcium channel \\
\hline
\end{tabular}




\begin{tabular}{|c|c|}
\hline LVEF & Left ventricular ejection fraction \\
\hline M & Mol per liter \\
\hline $\mathrm{Mb}$ & Megabase \\
\hline MDa & Megadalton \\
\hline MEF & Mouse embryonic fibroblast \\
\hline Mef2c & Myocyte enhancer factor 2C \\
\hline $\mathrm{mg}$ & Milligram \\
\hline $\mathrm{MgCl}_{2}$ & Magnesium chloride \\
\hline mGSCs & Multipotent germline stem cells \\
\hline $\min$ & Minute \\
\hline $\mathrm{mL}$ & Milliliter \\
\hline MLC2a & Myosin regulatory light chain 2 atrial isoform \\
\hline MLC2v & Myosin regulatory light chain 2 ventricular isoform \\
\hline $\mathrm{mm}$ & Millimeter \\
\hline MMC & Mitomycin \\
\hline $\mathrm{MnCl}_{2}$ & Manganese chloride \\
\hline MOI & Multiplicity of infection \\
\hline mRNA & Messenger RNA \\
\hline $\mathrm{ms}$ & Milliseconds \\
\hline MTG & 1-Thioglycerol \\
\hline $\mathrm{mV}$ & Millivolt \\
\hline MYH6/7 & Myosin heavy chain isoform 6/7 \\
\hline N2B & Small titin isoform \\
\hline N2BA & Large titin isoform \\
\hline $\mathrm{Na}^{+}$ & Sodium ion \\
\hline $\mathrm{Na}_{3} \mathrm{VO}_{4}$ & Sodium orthovanadate \\
\hline $\mathrm{NaCl}$ & Sodium chloride \\
\hline NADPH & Nicotinamide adenine dinucleotide phosphate \\
\hline $\mathrm{NaF}$ & Sodium fluoride \\
\hline $\mathrm{NaOH}$ & Sodium hydroxide \\
\hline NCF & Neutrophil oxidase factor \\
\hline NCX & $\mathrm{Na}+/ \mathrm{Ca} 2+$ exchanger \\
\hline NEAA & Non-essential amino acid \\
\hline
\end{tabular}


NFATC4 Nuclear factor of activated T-cells, cytoplasmic, calcineurin-dependent 4

ng Nanogram

nm Nanometer

NOX1-5 NADPH oxidase isoform 1-5

NRG1 Neuregulin-1

$\mathrm{O}_{2} \quad$ Molecular oxygen

OCT4 Octamer binding transcription factor 4

Orp1 Oxidant receptor protein-1

OxD Degree of oxidation

P/S Penicillin / streptomycin

PCR Polymerase chain reaction

PFA Paraformaldehyde

$\mathrm{pH} \quad$ Potential of hydrogen

Phox Phagocyte oxidase

PI Propidium iodide

PKA Protein kinase A

PLN Phospholamban

ps Patient-specific

PSC Pluripotent stem cell

PTPN11 Tyrosine-protein phosphatase non-receptor type 11

$\mathrm{R} \quad$ Arginine

RAC1/2 Ras-related C3 botulinum toxin substrate 1/2

RAN Ranolazine

redox Reduction-oxidation

Rev Reverse primer

RICOVER60 Rituximab with CHOP over age 60 years

RNA Ribonucleic acid

roGFP2 Reduction-oxidation-sensitive GFP

ROS Reactive oxygen species

rpm Rounds per minute 
rS

RT-PCR

RYR2

S16

S2808

S2814

SCN5

SDS

sec

SEM

SERCA

SNP

SOD

SOX2

SR

SRY

SSEA-4

Stat3

$\mathrm{T}$

T-X-100

$\mathrm{T} / \mathrm{E}$

$\mathrm{T}_{\mathrm{A}}$

TB-buffer

TBST

Tbx5

TEMED

TGF $\beta$

$\mathrm{TH}$

Thr17

TNFR1
Reference SNP ID number

Reverse transcriptase polymerase chain reaction

Ryanodine receptor isoform 2

Serine at position 16 of PLN aa sequence

Serine at position 2808 of RYR2 aa sequence

Serine at position 2814 of RYR2 aa sequence

Sodium voltage-gated channel alpha subunit 5

Sodium dodecyl sulfate

Seconds

Standard error of mean

Sarco/endoplasmic reticulum Ca2+-ATPase

Single nucleotide polymorphism

Superoxide dismutase

SRY-box2

Sarcoplasmic reticulum

Sex-determining region of $\mathrm{Y}$

Stage specific embryonic antigen 4

Signal transducer and activator of transcription 3

Thymine

Triton-X-100

Trypsin/EDTA

Annealing temperature

Tris/boride buffer

Tris-buffered saline with Tween20

T-box transcription factor-5

Tetramethylethylenediamine

Transforming growth factor type $\beta$

Tyrosine hydroxylase

Threonine at position 17 of PLN aa sequence

Tumor necrosis factor receptor 1 


$\begin{array}{ll}\text { TOP2 } \alpha / \beta & \text { Topoisomerase II isoform alpha/beta } \\ \text { TRA-1-60 } & \text { Tumor rejection antigen 1-60 } \\ \text { TZV } & \text { Thiazovivin } \\ \text { U } & \text { Unit } \\ \text { UV } & \text { Ultraviolet } \\ \text { V } & \text { Volt } \\ \alpha-\text { MHC } & \alpha \text { myosin heavy chain } \\ \alpha-S M A & \alpha \text { smooth muscle actin } \\ \beta \text {-III-Tub } & \beta \text {-III-Tubulin } \\ \beta-\text { ME } & \beta \text {-mercaptoethanol } \\ \mu \mathrm{g} & \text { Microgram } \\ \mu \mathrm{L} & \text { Microliter } \\ \mu \mathrm{m} & \text { Micrometer }\end{array}$




\section{Summary}

The anthracycline doxorubicin (DOX), one of the most effective chemotherapeutic drugs for the treatment of various cancers, is limited in its clinical applications due to cumulative dosedependent cardiotoxicity. The mechanisms of anthracycline-induced cardiotoxicity (ACT) and potential risk factors are still not fully understood. There is good evidence that the pathophysiology of ACT is multifactorial. Increased production of reactive oxygen species (ROS), topoisomerase II poisoning, disturbances in calcium signaling and sarcomere disarray are discussed as key pathomechanisms of ACT in cardiomyocytes. Recent studies suggest that single nucleotide polymorphisms (SNPs) in genes encoding for NADPH oxidase subunits are associated with the risk to develop ACT. In this study, we aimed to establish a human model of ACT. For this purpose, we used human induced pluripotent stem cells (hiPSCs) as a powerful means to analyze the cardiac phenotype of ACT and to investigate potential genetic risk factors.

Integration-free hiPSCs were generated from five patients who were treated with DOX as part of chemotherapy. Three patients with SNPs in the NADPH oxidase subunits RAC2 and p22phox developed ACT. Two patients without these SNPs did not develop ACT and were used as controls (Ctrl). The generated hiPSCs met criteria for pluripotency and were directly differentiated into cardiomyocytes (iPSC-CMs) with high purity. We analyzed the expression of NADPH oxidase subunits, generation of ROS, calcium homeostasis, apoptosis, sarcomeric integrity and mechanical functionality in Ctrl- and ACT-iPSC-CMs on the basal level and upon DOX application. We found a dose-dependent increase of oxidative stress upon DOX treatment in Ctrl- and ACT-iPSC-CMs using Amplex Red and genetically encoded sensors Grx1-roGFP2 and roGFP2-Orp1. The amount of ROS was significantly higher in ACT-iPSC-CMs upon treatment with $0.5 \mu \mathrm{M}$ DOX. Furthermore, DOX application caused disturbances in iPSC-CM calcium transients in both groups. Low and high DOX concentrations had contradicting effects on calcium transients indicating a biphasic mechanism. DOX application resulted in a significantly higher increase of apoptosis in ACT-iPSC-CMs compared to CtrliPSC-CMs. Furthermore, the sarcomeric integrity was significantly decreased in ACT-iPSC-CMs but not in Ctrl-iPSC-CMs upon treatment with clinically relevant DOX concentrations. Engineered heart muscles (EHMs) were generated from Ctrl- and ACT-iPSC-CMs to analyze mechanical functionality. DOX application caused an increase in EHM beating frequency and arrhythmia in both groups. Importantly, the maximal force of contraction decreased more in ACT-EHMs than in Ctrl-EHMs upon DOX application.

Taken together, we established a human iPSC-CM-based model of ACT that recapitulates critical pathomechanisms. Our findings indicate that the genetic background of chemotherapy patients determines the risk to develop ACT. In the future, the generated 
iPSC-CM ACT model may be used to analyze the mechanisms of ACT in a human cardiac context, to screen and develop cardioprotectants and to find new biomarkers of ACT. 


\section{Introduction}

\subsection{Anthracycline-induced cardiotoxicity}

Anthracyclines are a class of antineoplastic drugs, which are commonly used in chemotherapy. Doxorubicin (DOX) is one of the most prominent anthracyclines and was first isolated in the 1960s from Streptomyces peucetius (Arcamone et al., 1969; Di Marco et al., 1981). It is very effective against a broad range of solid and haematopoietic cancers and therefore has been a part of chemotherapy for over five decades. Thereby, it contributed to the overall increase in 5-year survival rate from $35 \%$ in the 1950 s to $70 \%$ in $2006-2012$ (Magdy et al., 2016).

Despite its widespread application, the precise mechanism of action of DOX in cancer cells is still not fully understood, but it is evident that the topoisomerase II (TOP2) is a main target. Further distinct pathways are also discussed which all result in apoptosis and cell death. The great effectivity of DOX against cancer cells may be attributed to this multifactorial mode of action. DOX binds to the alpha and beta isoform of TOP2, an enzyme that is located in the cell nucleus and untangles the genomic DNA during replication and translation. By binding to TOP2, DOX stabilizes an intermediate reaction complex of covalently linked TOP2 and cleaved DNA strands. Thereby, resealing of DNA strands is prevented, ultimately leading to DNA double-strand breaks and subsequent growth arrest and apoptosis (Chen, 2012; Hong et al., 1990; Tewey et al., 1984). Because of this mechanism, DOX is also classified as TOP2 poison, contrary to TOP2 inhibitors, which inhibit ATPase activity or trap the enzyme and thereby interrupt DNA binding. Cancer cells are especially affected by this mechanism of action, since the alpha isoform (TOP2 $\alpha$ ) is highly expressed in proliferative cells but not in quiescent cells.

DOX further intercalates into double-stranded DNA, forms DNA crosslinks and alkylates DNA, causing inhibition of DNA replication and protein biosynthesis. The formation of reactive oxygen species (ROS) may be another mechanism of DOX activity in tumor cells (Minotti et al., 2004). Metabolism of DOX and the formation of a complex with intracellular molecular iron results in the formation of ROS and will be discussed in detail in chapter 1.1.1.1. A high amount of ROS can cause direct cellular damage such as DNA damage and lipid peroxidation of organelle membranes. A milder increase of ROS also causes impairments in cell functionality since tightly controlled redox signaling pathways can get disturbed. The direct involvement of ROS in antitumor activity has been doubted because respective in vitro experiments were performed with DOX concentrations that were considerably higher than clinical concentrations. Additionally, the antitumor activity of DOX is partly mediated by the S26 proteasome. DOX binds to the allosteric site of the proteasomal S20 core particle and 
acts as a reversible noncompetitive inhibitor causing ubiquitinated proteins to accumulate (Minotti et al., 2004).

In spite of being highly effective in the treatment of cancer, the clinical use of DOX is limited by severe adverse drug events. Of these, anthracycline-induced cardiotoxicity (ACT) is the most prominent one and was first described in 1971 (Middleman et al., 1971). No clear definition of ACT exists because clinical studies vary broadly in cancer types, DOX application, detection methods and follow-up procedures. It may be defined "as subclinical or clinical, causing manifestations that include disturbance in ventricular de-/repolarization and QT interval, arrhythmia, bradycardia, tachycardia, decrease in left ventricular ejection fraction (LVEF) and fractional shortening (FS) and irreversible congestive heart failure (CHF), all of which lead to increased morbidity and mortality" (Magdy et al., 2016). Commonly, ACT is described as a type 1 cardiotoxicity that is dose-dependent, irreversible and caused by cardiomyocyte (CM) death. Furthermore, three types of ACT can be distinguished according to the time of occurrence and severity, namely the acute form, the early-onset chronic progressive and the late-onset chronic progressive form. The acute form of ACT is characterized by mostly mild symptoms such as transient rhythm disturbances or low blood pressure. Acute ACT occurs during or immediately after DOX application and disappears after treatment with antiarrhythmic drugs (Steinberg et al., 1987). The chronic form of ACT, however, is clinically relevant, characterized by the aforementioned symptoms and can be lethal. It can have an early onset directly after chemotherapy or a late onset years to even decades later (Steinherz et al., 1991). It is accompanied by ultrastructural changes like myofibril loss, sarcoplasmic reticulum (SR) dilation, cytoplasmic vacuolization, swelling of mitochondria and increase in lysosome number (Minotti et al., 2004). More recently, the paradigm that an acute form and chronic irreversible forms of ACT exist has been challenged. A potential for reversibility has been proposed in a prospective study with 2625 patients (Cardinale et al., 2015; Groarke and Nohria, 2015). With advances in detection of cardiotoxic effects, it becomes clear that ACT is not separable into three independent diseases. It may rather be a single progressing phenomenon, which previously has been detected in different stages. The earlier assumption might has been a result of the fact that most studies are retrospective, meaning most of the cardiac cases were found on random evaluations or when clinical symptoms already developed. When diagnosed and treated early, ACT might be reversible.

The estimation of ACT prevalence is hampered by the unclear definition, varying detection methods and differences in cancer type, DOX application and follow-up time used in clinical studies. The incidence is especially influenced by the cumulative DOX dose, which was already found in early studies. In 399 cancer patients, CHF was diagnosed in $4 \%, 18 \%$ or $36 \%$ of patients who received cumulative DOX doses of 501-550, 551-600 or over $600 \mathrm{mg} / \mathrm{m}^{2}$, 
respectively. No patient that received less than $501 \mathrm{mg} / \mathrm{m}^{2}$ DOX developed CHF (Lefrak et al., 1973). Further studies with 630 and 3941 patients report an amount of DOX-induced CHF of 3 and $5 \%, 7$ and $26 \%$ and 18 and $48 \%$ after cumulative doses of 400,550 and $700 \mathrm{mg} / \mathrm{m}^{2}$, respectively (Swain et al., 1997; Von Hoff et al., 1979). As a result, the life-long cumulative DOX dose is now set to $500 \mathrm{mg} / \mathrm{m}^{2}$, which unfortunately also limits neoplasticity. However, cardiotoxicity is still present at low doses. For example, $5.1 \%$ of patients in a retrospective study showed evidences of CHF (Swain et al., 2003) and in a more recent study the incidence of ACT was 9\% (Cardinale et al., 2015). A meta-analysis including 18 studies and 49,017 patients reported $6 \%$ clinical and $18 \%$ subclinical cardiotoxicity after a median follow-up of nine years (Lotrionte et al., 2013).

\subsubsection{Pathomechanisms of ACT}

Similar to the anticancer activity of DOX, the pathophysiology of ACT is multifactorial. As a result of an unclear definition of $\mathrm{ACT}$, the use of different in vitro and in vivo models and the varying methodology and experimental designs, the current state of knowledge is confusing and at times contradictory. However, a consistent result of most of the suggested pathomechanisms is loss of CMs by apoptosis, which cannot be compensated by the heart. Additionally, pathomechanisms that disturb the overall integrity of CMs have also been suggested. Below, the most commonly accepted pathomechanisms will be described.

\subsubsection{Increased ROS production}

The term ROS loosely describes a variety of reactive molecules or free-radicals derived from oxygen. The one electron reduction product of molecular oxygen, superoxide radical $\left(\cdot \mathrm{O}_{2}{ }^{-}\right)$, is one of the most important ROS in the cell as it serves as a progenitor for the majority of ROS. For example, dismutation of $\cdot \mathrm{O}_{2}^{-}$produces hydrogen peroxide $\left(\mathrm{H}_{2} \mathrm{O}_{2}\right)$, which in turn can generate the hydroxyl radical $(\cdot \mathrm{OH})$. Highly reactive ROS such as $\cdot \mathrm{OH}$ and peroxynitrite are usually short-lived and contribute to the overall oxidative stress by irreversible modifications of molecules. $\mathrm{O}_{2}{ }^{-}$and $\mathrm{H}_{2} \mathrm{O}_{2}$ are also involved in pathological oxidative stress. However, they are less reactive and therefore involved in reversible, physiological signaling processes. Contrary to highly reactive ROS, $\mathrm{O}_{2}^{-}$and $\mathrm{H}_{2} \mathrm{O}_{2}$ are generated enzymatically, are highly compartmentalized and modulate many cell signaling pathways by oxidation. This complex process is termed redox signaling and it is essential for normal cardiovascular physiology. Compartmentalization is partially achieved by specific localization of proteins, which remove ROS, such as superoxide dismutase (SOD) and catalases. Localization of ROS-generating enzymes to organelles or microdomains contribute to ROS compartmentalization. In the physiological context of CMs, ROS contribute to the excitation-contraction coupling by targeting key proteins in $\mathrm{Ca}^{2+}$ handling, such as the protein kinase A (PKA), Ca2+/calmodulin- 
dependent protein kinase II (CamKII), ryanodine receptor 2 (RYR2), the sarco/endoplasmic reticulum Ca2+-ATPase (SERCA) and phospholamban (PLN) (Burgoyne et al., 2012). Furthermore, ROS are involved in the proliferation and differentiation of CMs (Buggisch et al., 2007; Hom et al., 2011). Hence, dysregulation of redox signaling has been linked to various cardiac heart conditions, such as contractile dysfunction, hypertrophy, CM death, alterations in gene expression, arrhythmia and chamber dilation (Burgoyne et al., 2012; Kwon et al., 2003; Sabri et al., 2003; Siwik et al., 1999).

The production of ROS combined with resulting oxidative stress and cellular damage is one of the most widely accepted hypothesis for ACT (Angsutararux et al., 2015). On one hand, highly reactive ROS such as $\cdot \mathrm{OH}$ may cause ACT by directly damaging DNA, RNA, proteins and lipids. However, the aforementioned pathological consequences of more subtle dysregulation of redox cycling have also been implicated in ACT. As in cancer cells, DOX can increase ROS in CMs in two ways (Figure 1). First, it can be reduced to a semiquinone, which in turn auto-oxidates and forms $\cdot \mathrm{O}_{2}^{-}$. This redox cycling is catalyzed by flavoproteins, which transfer electrons from nicotinamide adenine dinucleotide phosphate (NADPH) to DOX, such as NADPH oxidases, NADPH-P450 reductase, xanthine oxidase, NO synthase and complex I of the mitochondrial electron chain (Davies and Doroshow, 1986; Doroshow, 1983). Dismutation of $\cdot \mathrm{O}_{2}^{-}$produces $\mathrm{H}_{2} \mathrm{O}_{2}$, which in turn can generate toxic $\cdot \mathrm{OH}$, a reaction catalyzed by iron (Štěrba et al., 2013). In the second mechanism, DOX directly forms a complex with molecular iron causing an iron cycling between $\mathrm{Fe}(\mathrm{II})$ and $\mathrm{Fe}(\mathrm{III})$ and $\cdot \mathrm{O}_{2}^{-}$production. This reaction is catalyzed by NADH cytochrome P450 reductases or thiols (Xu et al., 2005). The DOX-Fe(II) complex can also form $\cdot \mathrm{OH}$ by reduction of $\mathrm{H}_{2} \mathrm{O}_{2}$ and it can auto-oxidate into a free-radical, which in turn reacts with $\mathrm{O}_{2}$ to $\cdot \mathrm{O}_{2}^{-}$(Štěrba et al., 2013). 


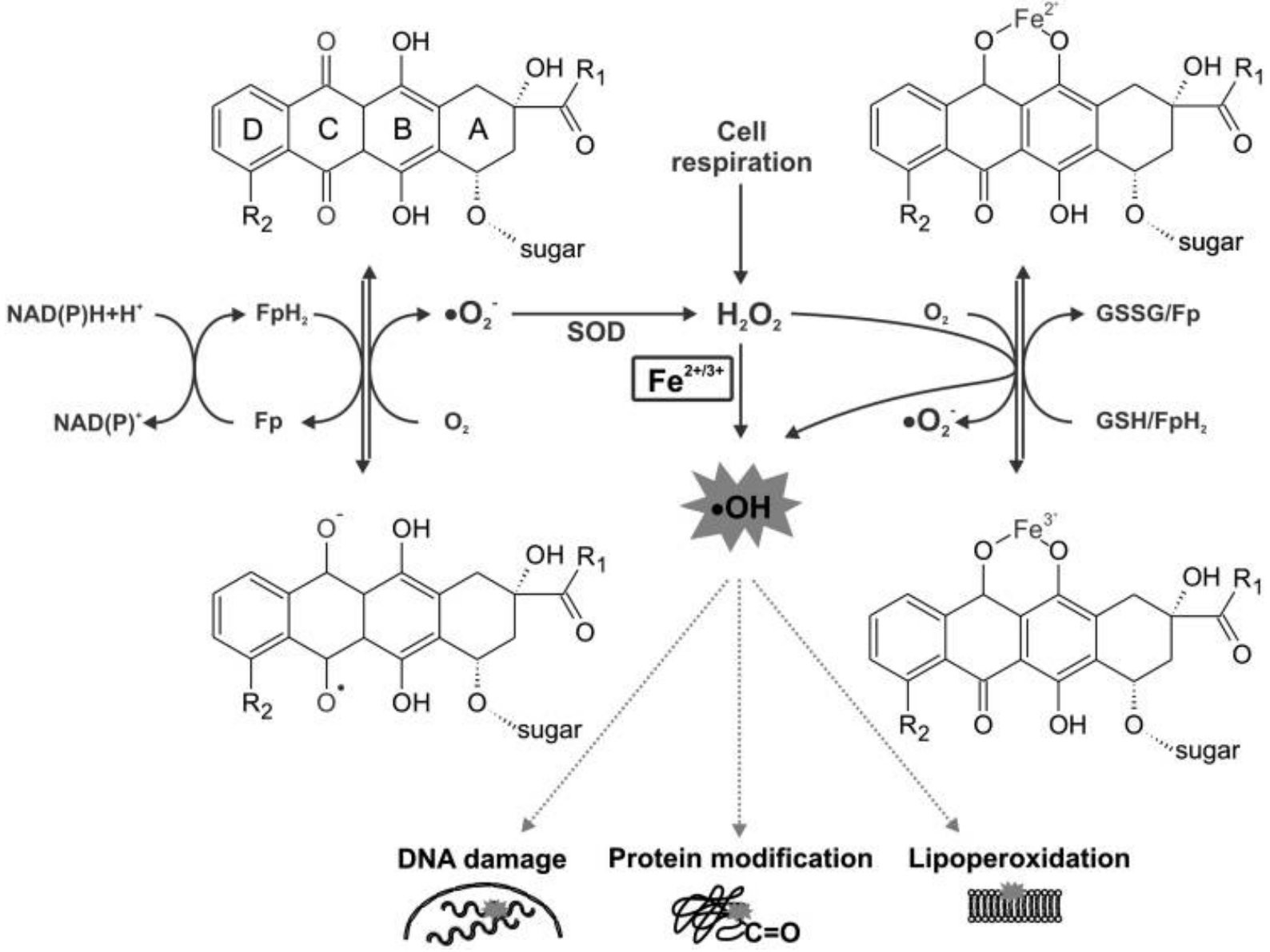

Figure 1: Schematic illustration of anthracycline-dependent ROS production. The top left molecular structural formula depicts the basic structure of anthracyclines. The left side shows the redox cycling of the quinone and semiquinone form of anthracyclines, which is catalyzed by flavoproteins (Fp) and generates $\mathrm{O}_{2}{ }_{2}$. The right side illustrates iron-cycling of anthracycline-iron complexes. Flavoproteins and thiol-containing compounds such as glutathione (GSSG/GSH) catalyze this $\cdot \mathrm{O}_{2}$ ' generating process. SOD generates $\mathrm{H}_{2} \mathrm{O}_{2}$ from $\cdot \mathrm{O}_{2}^{-}$. Subsequently, highly reactive $\cdot \mathrm{OH}$ can be formed during the HaberWeiss reaction of $\mathrm{H}_{2} \mathrm{O}_{2}$, which can be catalyzed by molecular iron. From Štěrba et al., 2013.

As stated above, NADPH oxidases take part in ROS-producing redox cycling of DOX. There is accumulating evidence to support a particular important role of NADPH oxidases in ACT (Gilleron et al., 2009; McLaughlin et al., 2017; Wojnowski et al., 2005; Zhao et al., 2010). NADPH oxidases are multi-subunit enzymes, which sole purpose is the production of ROS (Brandes et al., 2014). Thereby, they contribute to the tightly regulated redox signaling. Seven isoforms are reported and two are expressed in the heart, namely isoform 2 and 4 (Figure 2). In both isoforms, the transmembrane proteins NOX and p22phox form a heterodimer. The isoform-specific NOX is the catalytic subunit and p22phox is a scaffold protein. NOX2 and NOX4 share about $60 \%$ of their sequence, resulting in significant differences in activity and localization. NADPH oxidase 2 activation relies on RAC1 or RAC2 and a complex of p40phox, p47phox and p67phox, which are all cytosolic proteins. NADPH oxidase 2 produces $\cdot \mathrm{O}_{2}{ }^{-}$and is localized mainly in the cell membrane (Lassègue et al., 2012). NADPH oxidase 4 , on the other hand, produces $\mathrm{H}_{2} \mathrm{O}_{2}$ and is constitutively active, meaning 
that it is only regulated by its biosynthesis. It has been reported to be localized in intracellular membranes of organelles such as mitochondria, the endoplasmic reticulum and the nucleus (Anilkumar et al., 2013; Block et al., 2009; Lassègue et al., 2012). Two research groups could show that NOX2 depletion in mice resulted in attenuated DOX-induced damage (Wojnowski et al., 2005; Zhao et al., 2010), which was further validated in vitro (Gilleron et al., 2009; Ma et al., 2013).

NADPH oxidase 2

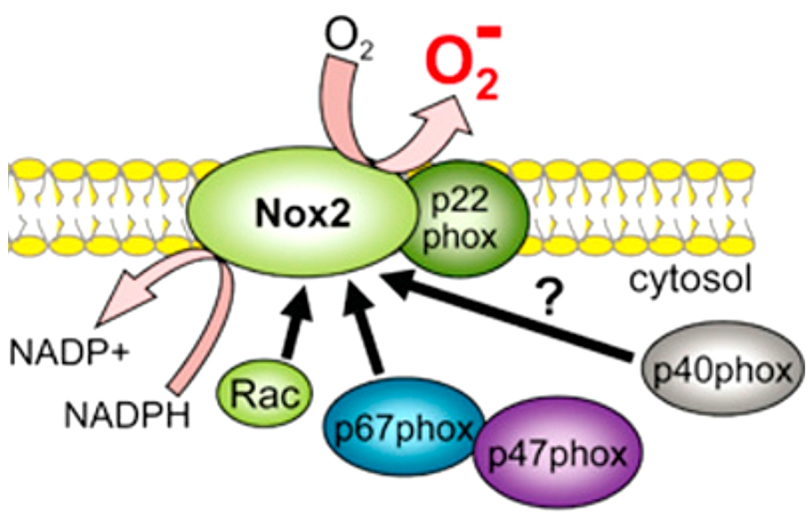

NADPH oxidase 4

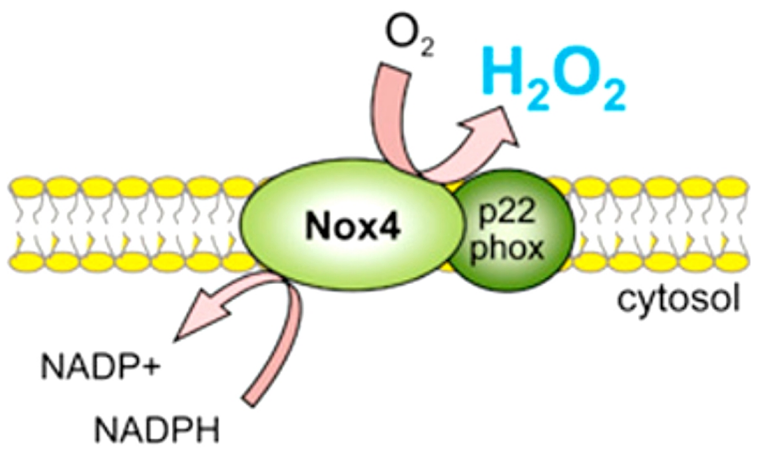

Figure 2: Schematic illustration of multi-subunit complexes NADPH oxidase 2 and 4 . In both isoforms, NOX and p22phox form a heterodimer, which is membrane-located. NADPH oxidase 2 is regulated by RAC1/2, p40phox, p47phox and p67phox. The influence of p40phox is still controversial, as it likely activates and inhibits the enzyme depending on the situation (illustrated by a question mark). NADPH oxidase 4 is constitutively active. From Brandes et al., 2014.

Mitochondria are especially involved in DOX-induced ROS production and are also one of their main targets (Berthiaume and Wallace, 2007; Gratia et al., 2012; Štěrba et al., 2011; Wallace, 2003). DOX accumulates in these organelles due to a high affinity for cardiolipin, which is part of the inner mitochondrial membrane (Goormaghtigh et al., 1990). Thereby, it gets in close proximity to complex I of the electron transport chain and to NADPH oxidase 4. Furthermore, DOX also causes molecular iron to accumulate in mitochondria, further increasing ROS production (Ichikawa et al., 2014). By the partial diversion of electrons from the electron chain, DOX may increase the basal respiration state (Gosalvez et al., 1974). Further impairments of the respiratory chain have been reported, but it remains unclear, if they are directly induced by DOX or a consequence of previous damage (Ohkura et al., 2003; Oliveira and Wallace, 2006; Yen et al., 1999). Mitochondria contain their own genome, which is damaged by DOX-induced oxidation (Serrano et al., 1999).

Although DOX-induced ROS production is also discussed as a mechanism of action in cancer cells and should happen in other cell types as well, CMs are suggested to be especially vulnerable to oxidative stress. This can be attributed to their dependency on mitochondria, 
which are highly dense in CMs and seem to be at the same time the main source and target of DOX-induced ROS. It was shown that cardiac mitochondrial DNA is more impaired by DOX than liver mitochondrial DNA (Serrano et al., 1999). Furthermore, it is claimed that the endogenous antioxidant state in CMs is lower in comparison to other cell types (Doroshow et al., 1980), which would be a reason for their vulnerability, but little convincing evidence actually seems to exist. Interestingly, it was shown that parts of the oxidative stress defense system are inhibited upon DOX application (Li et al., 2002; Siveski-Iliskovic et al., 1995).

Experimental studies show conflicting results regarding the oxidative stress hypothesis, since treatment with antioxidants decreased DOX-induced cell damage in some cases (Doroshow et al., 1981; Ichihara et al., 2007; Myers et al., 1977), but also had no protective effect in other cases (Berthiaume et al., 2005; Herman et al., 2000). In clinical trials, antioxidants such as vitamin $\mathrm{E}, \mathrm{N}$-acetylcysteine and 7-monohydroxyethylrutoside could not protect against ACT (Bruynzeel et al., 2007; Dresdale et al., 1982; Legha et al., 1982).

\subsubsection{Topoisomerase II poisoning}

Contrary to TOP $2 \alpha$, the beta isoform (TOP2 $\beta$ ) is ubiquitously expressed in quiescent cells such as CMs (Capranico et al., 1992). Since no significant clinical translation of the oxidative stress hypothesis could yet be achieved, TOP $2 \beta$ poisoning of DOX is discussed as alternative explanation for ACT. The best experimental evidence was reported by Zhang et al. who showed that CM-specific depletion of Top $2 b$ protected mice from developing ACT (Zhang et al., 2012). Furthermore, Top $2 b$ depletion in CMs resulted in less DNA double-strand breaks upon DOX application and reduced changes in expression of genes, responsible for mitochondrial functionality. Importantly, DOX-induced mitochondrial damage and ROS production was also decreased in Top $2 b$ depleted CMs, suggesting that these are secondary mechanisms and not the primary cause of ACT. This theory is supported by the findings that dexrazoxane (DEX), which is an iron chelator and TOP2 inhibitor, protects against ACT (see 1.1.2) but other more efficient metal chelators do not (Martin et al., 2009). Isoform-specific inhibition of TOP $2 \beta$ seems to be a promising way to protect against ACT without hampering antineoplastic effects. Clinical application and conformation of the theory is still missing.

\subsubsection{Disturbance of Calcium signaling}

Calcium ion $\left(\mathrm{Ca}^{2+}\right)$ homeostasis is critical for $\mathrm{CM}$ functionality since it determines contraction and relaxation, electrophysiological properties and gene expression. The excitationcontraction coupling in $\mathrm{CMs}$ is based on $\mathrm{Ca}^{2+}$-induced $\mathrm{Ca}^{2+}$ release (Figure 3 ). In this mechanism, an action potential activates voltage-dependent L-type $\mathrm{Ca}^{2+}$ channels in the transverse T-tubular membrane. This causes small amounts of extracellular $\mathrm{Ca}^{2+}$ to enter the cytosol and to bind and activate the SR-located RYR2, which is a $\mathrm{Ca}^{2+}$ release channel. Large 
amounts of $\mathrm{Ca}^{2+}$ are subsequently released from the SR into the cytoplasm, which ultimately leads to muscle contraction. Muscle relaxation is achieved by the recycling of $\mathrm{Ca}^{2+}$ into the $\mathrm{SR}$ by SERCA or by the transport out of the cell by the $\mathrm{Na}^{+} / \mathrm{Ca}^{2+}$ exchanger (NCX) (Eisner et al., 2017).

RYR2 is located in the SR in close proximity to T-tubules, which are part of the cell membrane and rich in voltage-dependent $\mathrm{Ca}^{2+}$ channels like the L-type calcium channel. It is part of a multiprotein complex that governs its channel open probability according to the amount of SR-located $\mathrm{Ca}^{2+}$ (Bers, 2004; Zalk et al., 2007). Two sites have been described, which activate RYR2 upon phosphorylation, namely S2808 and S2814. According to common opinion, S2808 is a target of the PKA (Lundby et al., 2013; Marx et al., 2000). Thereby, RYR2 activity is directly linked to $\beta$-adrenergic signaling as part of the fight-or-flight response. S2814, on the other hand, is mainly phosphorylated by CamKII (Ai et al., 2005). Since CamKII is $\mathrm{Ca}^{2+}$ dependent and targets RYR2 subsequently to the PKA, S2814 phosphorylation is thought of as a positive feedback loop. Additionally, PKA and CamKII can be activated in a CAMP and $\mathrm{Ca}^{2+}$ independent manner by oxidation (Burgoyne et al., 2012; Erickson et al., 2008; Santos et al., 2016). RYR2 activity may be directly regulated by oxidation of thiol residues (Burgoyne et al., 2012; Santos et al., 2016). Thereby RYR2 activation is coupled directly to redox cycling.

SERCA is located at the SR membrane and it is directly activated by high concentrations of cytosolic $\mathrm{Ca}^{2+}$ (Hove-Madsen and Bers, 1993). Additionally, SERCA Ca ${ }^{2+}$ affinity and thereby its activity is reduced by PLN. PLN, on the other hand, is inhibited by phosphorylation of the sites S16 and Thr17 by PKA and CamKII, respectively. As a result, SERCA activity is enhanced. Similar to RYR2 phosphorylation, PLN targeting by PKA and CamKII links SERCA activity to $\beta$-adrenergic-, $\mathrm{Ca}^{2+}-$ and redox signaling. Furthermore, SERCA activity may be directly regulated by oxidation of thiol residues (Burgoyne et al., 2012; Santos et al., 2016). NCX function and regulation is more complex. It is located in the cell membrane and exchanges three molecules of sodium $\left(\mathrm{Na}^{+}\right)$for one molecule of $\mathrm{Ca}^{2+}$. The direction of exchange is determined by the electrochemical gradients of $\mathrm{Na}^{+}$and $\mathrm{Ca}^{2+}$ and predominately $\mathrm{Ca}^{2+}$ is excluded out of the cell (Bers et al., 2003). $\mathrm{Ca}^{2+}$ efflux by NCX is inhibited by high cytosolic $\mathrm{Na}^{+}$ concentrations. Additionally NCX is negatively regulated by phospholemman upon phosphorylation of the later by PKA and CamKII (Cheung et al., 2013; Wang et al., 2011). 


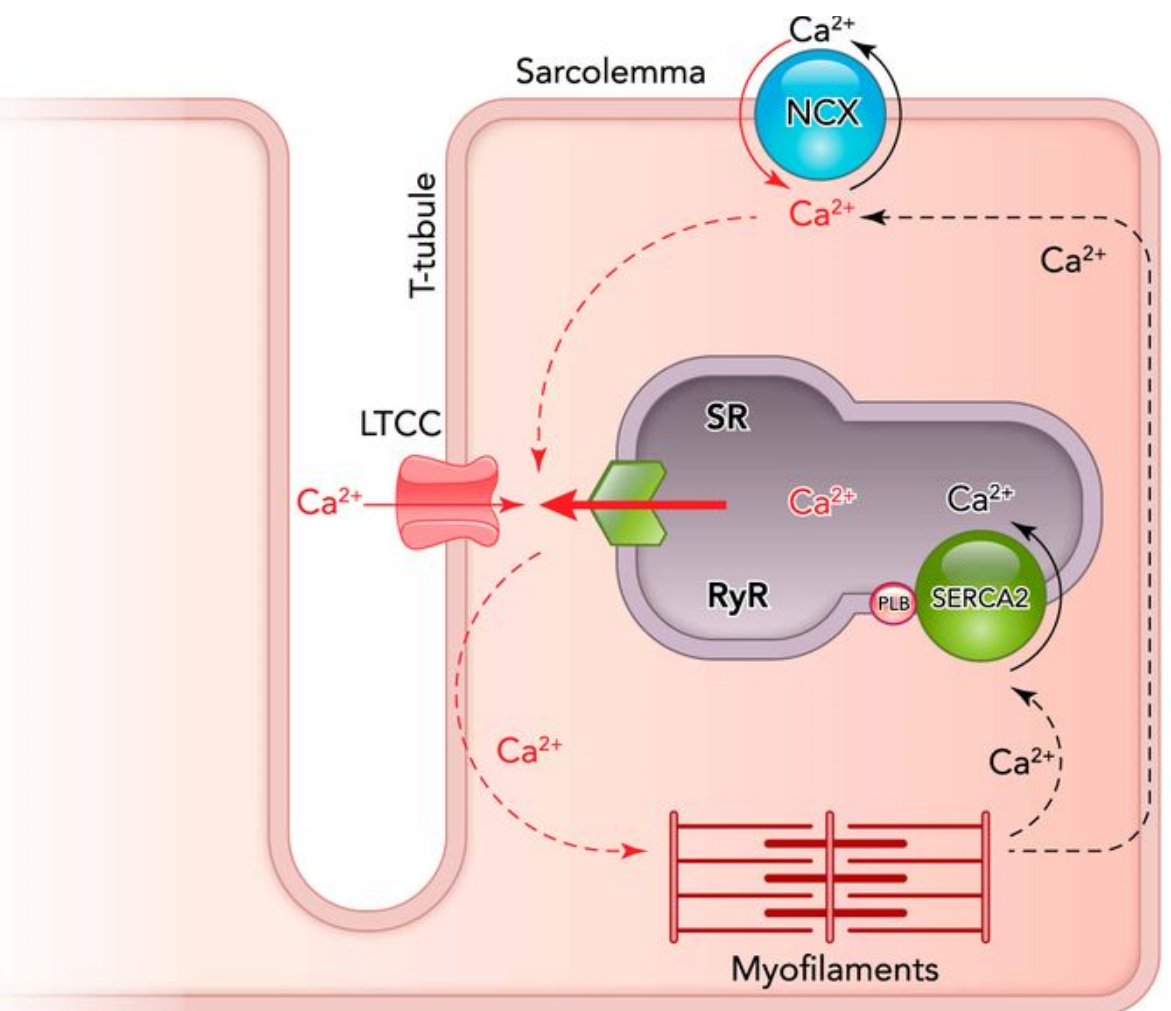

Figure 3: Schematic illustration of $\mathrm{Ca}^{2+}$ signaling in $\mathrm{CMs}$. Voltage dependent L-type $\mathrm{Ca}^{2+}$ channels (LTCC) in the transverse T-tubular membrane are activated by an action potential and a small amount of $\mathrm{Ca}^{2+}$ enters the cytoplasm. This activates the RYR2, which is located in the membrane of the SR and a large amount of $\mathrm{Ca}^{2+}$ enters the cytoplasm, causing contraction of myofilaments (depicted by red arrows). Subsequently, $\mathrm{Ca}^{2+}$ is transported into the SR or out of the cell by SERCA and NCX, respectively (depicted by black arrows). Phospholamban (PLB) regulates SERCA activity. From Shiels and Galli, 2014.

DOX application causes an increase of cytosolic $\mathrm{Ca}^{2+}$ in CMs (Kim et al., 2006; Sag et al., 2011), which is attributed to altered activity of $\mathrm{Ca}^{2+}$-channels. DOX binds to RYR2 and causes activation by increasing the channel open probability (Hanna et al., 2014; Kim et al., 1989; Ondrias et al., 1990; Zorzato et al., 1986) thereby causing more $\mathrm{Ca}^{2+}$ to enter the cytoplasm. SERCA activity, on the other hand, is reduced by DOX or its metabolites which is further aggravated by downregulation of SERCA gene expression (Hanna et al., 2014; Zhang et al., 2014). As a result, $\mathrm{Ca}^{2+}$ transport into the $\mathrm{SR}$ is decelerated and $\mathrm{Ca}^{2+}$ accumulates in the cytoplasm. Ultimately, increased cytosolic $\mathrm{Ca}^{2+}$ causes a stiffening of $\mathrm{CMs}$, since sarcomeres cannot fully relax anymore, which leads to a reduction of the hearts contractile force. Furthermore, changes in RYR2 and SERCA activity might cause arrhythmia, which could contribute to heart failure (Hanna et al., 2014).

\subsubsection{Disarray of sarcomeric cytoskeleton, NRG1/ErbB2 signaling and the influence of non-CMs}

It should be noted that additional pathomechanisms have been suggested, further complicating the situation. In most cases, it is not clear, if these are actual causes for ACT 
that are directly induced by DOX or if they are merely secondary results and thereby part of the accumulating cellular impairment. One of such is the disarray of the sarcomeric cytoskeleton, which is an early sign of ACT and can be observed in heart biopsies. The giant protein titin is of major importance for sarcomeric integrity, since it acts as a scaffold for sarcomere assembly and as a molecular spring, which contributes to diastolic function. It was shown that titin is actively degraded by calpain upon DOX application, causing myofibrillar disarray and necrosis of CMs (Lim et al., 2004). Because calpain is regulated by $\mathrm{Ca}^{2+}$, titin degradation is most likely a result of the disturbances of $\mathrm{Ca}^{2+}$ homeostasis, namely increased cytosolic $\mathrm{Ca}^{2+}$ concentration. Additionally, DOX-induced decrease in expression of sarcomeric genes have also been made responsible for loss of sarcomeric integrity. $\alpha$-actin, troponin I and myosin light chain 2 expression has been shown to be decreased upon DOX application in vitro and in vivo in mice, as well as the expression of CARP and GATA4, which are regulators of sarcomeric gene expression (Ito et al., 1990; Chen et al., 2012).

The growth factor neuregulin-1 (NRG1) and its tyrosine kinase receptors epidermal growth factor receptor 2 and 4 (ErbB2/4) are among the most important pro-survival signaling cascades in CMs and have been suggested to be involved in ACT. Depletion of ErbB2 or NRG1 in mice resulted in stronger ACT-associated symptoms upon DOX treatment in vitro and in vivo (Crone et al., 2002; Liu et al., 2005). Overexpression of ErbB2 or NRG1 treatment was beneficial in mice in vivo and in vitro (Belmonte et al., 2015; Bian et al., 2009). Interestingly, ErbB4 is downregulated via microRNA in hearts of mice after DOX treatment (Horie et al., 2010).

The majority of studies, which aim to reveal ACT pathomechanisms, focus on CMs. Yet, cardiac biology involves additional cell types, namely cardiac fibroblasts, endothelial cells, vascular smooth muscle cells and cardiac progenitor cells, all of them being involved in cardiac homeostasis. More recently, it was suggested that direct adverse effects of DOX on non-CMs contribute to ACT. For example, development of fibrosis may be amplified by cardiac fibroblasts upon DOX application (Cappetta et al., 2016) and inhibition of cardiac progenitor cells causes an impaired CM turn-over (De Angelis et al., 2010).

\subsubsection{Treatment and prophylactic therapy of ACT}

Despite their adverse drug events, anthracyclines still play a major role in common chemotherapy, with $32 \%$ of breast cancer patients (Giordano et al., 2012), 57 to $70 \%$ of elderly lymphoma patients (Chihara et al., 2016; Nabhan et al., 2015) and 50 to $60 \%$ of childhood cancer patients (Smith et al., 2010) receiving them. Few studies with sufficiently large amount of cases exists that report the mortality of ACT, however, the prognosis of patients who developed ACT is usually poor with 50-80\% (Lefrak et al., 1973; Von Hoff et al., 1979). No official guidelines or recommendations for the treatment of cardiac dysfunction 
specifically resulting from ACT are available (Volkova and Russell, 2011; Zamorano et al., 2016). Instead, usual therapy for heart failure unrelated to cardiotoxicity is applied such as ACE (angiotensin-converting enzyme)-inhibitor or $\beta$-blocker therapy (Yancy et al., 2013). Furthermore, permanent therapies such as heart transplantation or application of mechanical circulatory devices are often necessary.

Current strategies to reduce cardiotoxicity of DOX include prolongation of intravenous injection time and application of liposome-encapsulated DOX (Airoldi et al., 2011; van Dalen et al., 2009, 2010) but convincing long-term studies regarding to their effectiveness are missing. Much effort has been made to improve on prophylactic therapy of ACT, which aims to find drugs that could be applied before or during DOX treatment and act as protectant against ACT. Thereby, the anticancer activity of DOX would remain, whereas cardiotoxicity would decrease. So far, only one drug, DEX, has been approved as cardioprotective (van Dalen et al., 2008; Lipshultz et al., 2004, 2010). Data on other candidates such as statins, ACE-inhibitors, $\beta$-blockers, amifostine, acetylcysteine, vitamin $D$, calcium channel blockers, coenzyme Q10 and L-carnitine has been inconclusive (van Dalen et al., 2008; Smith et al., 2010). DEX is a derivative of EDTA and was first thought to convey cardioprotection against DOX by chelating metals such as iron, since iron dependent DOX-induced ROS production is thought of as a main pathomechanism of ACT (see chapter 1.1.1.1). However, only DEX metabolites, but not DEX itself, show high affinity for iron and are not cardioprotective by themselves (Hasinoff et al., 1998, 2003; Kaiserová et al., 2006). It is more likely that DEX binds TOP2 which is also a target of DOX. Whereas DOX stabilizes TOP2 after cleaving the DNA and thereby induces double-strand breaks, DEX binds TOP2 before DNA cleavage and blocks DOX binding. TOP 2 binding by DOX is also a main way of action in cancer cells. Due to this fact and two studies, which were already challenged, the United States Food and Drug Administration (FDA) restricted DEX to adult patients, who already received $300 \mathrm{mg} / \mathrm{m}^{2} \mathrm{DOX}$, because DEX potentially decreases anti-cancer activity of DOX (FDA, 2011). Recent in vitro experiments suggest further drugs to be potentially cardioprotective against DOX, including Ranolazin (RAN). The late sodium current inhibitor RAN is used to treat angina and has experimentally been reported to improve recovery after heart failure (Hwang et al., 2009). In mice and rat models, RAN decreased cardiotoxicity of DOX which was accompanied by reduction of $\mathrm{H}_{2} \mathrm{O}_{2}$ (Cappetta et al., 2017; Tocchetti et al., 2014).

\subsubsection{Pharmacogenetics of ACT}

As mentioned before (chapter 1.1), the risk to develop ACT depends on the cumulative dose. Apart from that, further risk factors for the development of ACT have been reported, such as female gender, African-American ethnicity, very young or old age or previous cardiac diseases (Lipshultz et al., 1995; Lotrionte et al., 2013). Importantly, these clinical and demographic risk 
factors still cannot explain the high interindividual variation of cardiotoxicity severity when similar DOX treatment schedules are applied. It seems that some individuals can survive high doses of DOX without cardiac conditions, whereas others develop ACT after application of low doses. Hence, a genetic predisposition for ACT has been postulated and studied intensely in the current decade.

A recent systematic review until May 2016 identified 28 independent studies which report on the association of genetic variants with ACT (Leong et al., 2017). According to the review, 84 genes and 147 single nucleotide polymorphisms (SNPs) have been investigated and 87 SNPs have been reported by at least one study to be significantly associated with ACT. The majority of this studies investigated candidate genes that are involved in anthracycline metabolism and handling, oxidative stress or general cardiac function. The authors were able to perform a meta-analysis of SNPs with those studies that provided sufficient data. Interestingly, only three SNPs significantly increased odds of cardiotoxicity in individuals treated with anthracyclines, namely $A B C C 2$ (ATP Binding Cassettes Subfamily C Member 2) rs8187710, CYBA (Cytochrome B-245 alpha chain) rs4673 and RAC2 rs13058338. ABCC2 belongs to a superfamily of transmembrane proteins, which transport substrates, including DOX, across the cell membrane using ATP. The rs8187710 SNP results in an amino acid change which has been shown to reduce ATPase activity and causes a reduced efflux activity (Elens et al., 2011). While a theoretical rationale is thereby given, no experimental data exists that shows a reduction of ACT by rs 8187710 . Both p22phox (encoded by CYBA) and RAC2 are part of NADPH oxidases (see chapter 1.1.1.1), with p22phox being an essential subunit of most isoforms and RAC2 being an activator of certain isoforms, such as NADPH oxidase 2. CYBA rs4673 causes histidine at position 72 of p22phox to be replaced by tyrosine. The molecular consequence of rs4673 remains elusive, since the p22phox histidine is not involved in binding of heme, which is part of the complex between p22phox and NOX (Biberstine-Kinkade et al., 2001). Furthermore, substitution of p22phox histidine with tyrosine does not influence NADPH oxidase functionality or complex stability (BiberstineKinkade et al., 2002). In neutrophils $\cdot \mathrm{O}_{2}^{-}$production was higher upon stimulation in the presence of the ACT-associated allele of rs4673 (Shimo-Nakanishi et al., 2004). On the other hand, vascular $\mathrm{O}_{2}^{-}$production has been reported to be reduced by the same allele of rs4673 (Guzik et al., 2000). rs8187710 of RAC2 is located in an intron and no direct link to the protein functionality has been reported. However, the gene expression of RAC2 and NCF4 (neutrophil oxidase factor4; encoding for the NADPH oxidase subunit p40phox) was increased in the presence of the ACT-associated allele of rs8187710 (Schirmer et al., 2007). Similar to $A B C C 2$ rs8187710, no experimental data regarding cardiotoxicity was reported for rs4673 and rs13058338. Both SNPs are located on independent genes that encode subunits of one enzyme complex. Additionally, NADPH oxidases are implicated in DOX-induced ROS 
production. For this reasons rs4673 and rs13058338 are particularly promising candidates for prognostic biomarkers of ACT outcome. In conclusion, various SNPs could be shown to be statistically associated with ACT, but no satisfying experimental data has yet been reported.

\subsection{Induced pluripotent stem cells in cardiovascular medicine}

\subsubsection{Characteristics of pluripotent stem cells}

Stem cells are characterized by the ability to self-renew through cell division and to differentiate into specialized cell types (National Institutes of Health, 2016). They can be classified according to their differentiation potential or to their origin (Jaenisch and Young, 2008). A totipotent cell is capable of producing all differentiated cells in an organism, including extraembryonic tissues. Only the zygote and the first cleavage blastomeres are totipotent (Kelly, 1977). Pluripotency is defined by the capacity to give rise to somatic cells of all three germ layers and the germ line. In vivo, pluripotent cells make up the inner cell mass of the blastocyst and later the epiblast. Several pluripotent cells of different origins have been successfully cultured, with embryonic stem cells (ESCs) being the most prominent ones. Stem cells that are found in adult stages, are multipotent or unipotent. They are necessary in the adult organism for tissue homeostasis and tissue regeneration after injury. Multipotent stem cells have the capacity to give rise to several differentiated cell types that belong to one cell lineage, for example hematopoietic stem cells, which are the origin of all blood cells. Spermatogonial stem cells and epidermal stem cells are examples for unipotent stem cells because they produce exclusively one cell type, sperm cells and keratinocytes, respectively. The differentiation potential of stem cells is entirely lost upon terminal differentiation.

Since pluripotent stem cells (PSCs) have the greatest developmental and therapeutic potential, they have been intensely investigated for five decades. The first pluripotent cell lines were established from teratocarcinomas, germ cell tumors, and were called embryonic carcinoma cells (ECCs) (Kleinsmith and Pierce, 1964; Stevens and Little, 1954). Although ECCs were used as a model for early mouse development, they are aneuploid and contribute only poorly to adult somatic tissue upon injection into blastocysts and transplantation into foster females (Brinster, 1974). In 1981 ESCs were first derived from the inner cell mass of mouse blastocysts (Evans and Kaufman, 1981; Martin, 1981) and subsequently from human embryos (Thomson et al., 1998). Both mouse and human ESCs are capable of efficiently differentiating into tissues of all germ layers and the germ line (Nagy et al., 1990), can be expanded in vitro without limitations and maintain a normal karyotype. Furthermore, ESCs are characterized by the expression of OCT4 (Octamer binding transcription factor 4), SOX2 (SRY-box2; SRY: Sex-determining region of Y) and NANOG, which are key pluripotency genes, 
and a high telomerase and alkaline phosphatase activity (Hanna et al., 2010b). Despite these similarities, there are major differences between human and mouse ESCs, for example, in the morphology. Mouse ESCs grow in domed and compacted colonies and human ESCs in flat colonies. Furthermore, the culture conditions that keep the ESCs in an undifferentiated state differ. Mouse ESCs depend on signaling through the Stat3 pathway, activated by leukemia inhibitory factor (LIF), while human ESCs depend on fibroblast growth factor-2 (FGF2)/Activin signaling (Hanna et al., 2010b). These differences reflect the fact that pluripotency itself can be classified into various grades of stringency, distinguishable by different in vivo functional assays. The naive state of ESCs corresponds to the inner cell mass of the primordial embryo, whereas the primed state resembles the postimplantation epiblast (Nichols and Smith, 2009; Theunissen et al., 2016). Traditionally, mESCs and miPSCs are described as naive, whereas hESCs and hiPSCs are primed, however, several groups have already reported the generation of naive hESCs (Gafni et al., 2013; Hanna et al., 2010a; Takashima et al., 2014; Theunissen et al., 2014; Ware et al., 2014). For this reasons, it can be difficult to determine, whether differences are based on variations between species or stages of pluripotency.

Pluripotent cell lines have been derived from other embryonic and adult tissues, such as epiblast-derived stem cells (EpiSCs) from post implantation embryos (Brons et al., 2007), embryonic germ cells (EGCs) from primordial germ cells (Matsui et al., 1992) and multipotent germline stem cells (mGSCs) from neonatal (Kanatsu-Shinohara et al., 2004) and adult mouse spermatogonial cells (Guan et al., 2006). PSCs provide a powerful tool to study early human development and hold great potential for clinical applications. However, major concerns about the use of hESCs are also discussed. The embryonic origin and the associated destruction of human embryos cause ethical and political problems. Adult stem cells on the other hand are difficult to isolate and to culture. Furthermore, potential tumorigenicity of PSCs and the immune rejection of stem cell-derived tissue grafts are issues that still need to be solved.

\subsubsection{Induced pluripotency}

The transfer of nuclei from somatic cells into oocytes (Briggs and King, 1952) and the fusion of somatic cells with embryonic stem cells (ESCs) (Tada et al., 2001) showed that terminally differentiated cells can be reprogrammed by factors contained by oocytes and ESCs. Furthermore, lineage conversion experiments were performed in which the ectopic expression of cell type-specific transcription factors switched one cell type into another (Xie et al., 2004). These findings motivated attempts to generate PSCs from somatic cells by reprogramming, using factors that are specifically expressed in ESCs or are important for the maintenance of their pluripotency. In 2006, PSCs were generated for the first time from mouse fibroblasts and named induced pluripotent stem cells (iPSCs) (Takahashi and 
Yamanaka, 2006). The fibroblasts were reprogrammed by retroviral transduction with the four transcription factors OCT3/4, SOX2, C-MYC and Krüppel-like factor 4 (KLF4). Subsequently, human iPSCs were generated from fibroblasts using the same combination of transcription factors (Takahashi et al., 2007) or different combinations like OCT4, SOX2, NANOG and LIN28 (Yu et al., 2007). IPSCs resemble ESCs in defining features like morphology, expression of pluripotency markers, self-renewal, teratoma formation and chimera development. In the past decade since first-generation iPSCs were reported, rapid progress was made concerning the reprogramming, culture conditions, safety and applications.

The molecular mechanisms behind pluripotency and reprogramming of somatic cells into iPSCs are complex and not fully understood. The transcription factor NANOG belongs to the key players of pluripotency and its disruption causes loss of pluripotency (Chambers et al., 2003; Mitsui et al., 2003). SOX2 and OCT4 are additional transcription factors that form a heterodimer (Masui et al., 2007; Nichols et al., 1998). Together with NANOG, these three transcription factors are thought to be the major regulators in a complex transcriptional network that maintains the pluripotency of a cell. They act both as activators of pluripotency genes and inhibitors of differentiation-related genes and often co-occupy their target genes. Furthermore, they activate their own gene expression in an autoregulatory loop (Boyer et al., 2005; Chen et al., 2008; Kim et al., 2008). LIN28 is among the activated target genes of NANOG, OCT4 and SOX2. It encodes for an RNA binding protein that inhibits the activity of let7 microRNA and thereby suppresses differentiation (Heo et al., 2009; Melton et al., 2010). Another transcription factor, which is in close functional relationship with the core transcription factors, is KLF4. It is involved in the self-renewal and maintenance of ESCs and regulates the expression of NANOG (Zhang et al., 2010). Furthermore, MYC was found to be involved in self-renewal and pluripotency (Cartwright et al., 2005). Additional markers for undifferentiated cells are the glycosphingolipid SSEA-4 and the Tra-1-60 antigen, which are located at the cell membrane. However, their function in pluripotency is not known (Brimble et al., 2007; Schopperle and DeWolf, 2007). Alkaline phosphatase is an enzyme that dephosphorylates nucleotides, proteins and alkaloids. Since its activity is high in undifferentiated cells, it can be used as a stem cell marker (O'Connor et al., 2008).

While the exact mechanisms remain elusive, reprogramming is thought of as a stochastic and deterministic mechanism with various events occurring sequentially or in parallel (Takahashi and Yamanaka, 2016). In the early phase of reprogramming, C-MYC binds to genomic loci with methylated histone 3 lysine 4 (H3K4) and thereby inhibits expression of somatic genes. Furthermore, the four reprogramming factors bind to enhancers and promoters of pluripotency-related genes and induce their expression (Soufi et al., 2012). In the second phase, the reprogramming factors activate further pluripotency-related genes, which were not accessible in the beginning (Buganim et al., 2012). As a result of the changes in gene 
expression, mesenchymal-to-epithelial transition and glycolysis-based metabolism is activated (Panopoulos et al., 2012; Samavarchi-Tehrani et al., 2010).

Various different approaches for the generation of iPSCs have been described. In the beginning, reprogramming factors were stably integrated into the genome of somatic cells by retroviral or lentiviral transduction. While being sufficient for iPSC generation, random integration of transgenes into the genome can lead to insertional mutagenesis and disrupt the functionality of neighboring genes. Furthermore, silencing of retroviral and lentiviral vectors occurs during reprogramming (Hotta and Ellis, 2008). Incomplete or untimely silencing may cause partial reprogramming with continued dependence on exogenous pluripotency genes, disturbance in the developmental potential and tumor formation in chimeras. To avoid these problems, inducible vector systems have been designed, which allow the control of transgene expression (Brambrink et al., 2008). An alternative approach is the use of integrating vectors with high reprogramming efficiencies that can be excluded from the genome subsequently to reprogramming. More recently, integration-free methods have been developed that rely on transient expression of reprogramming factors using adenoviral vectors (Stadtfeld et al., 2008) or sendai virus (Fusaki et al., 2009; Nishimura et al., 2011). The sendai virus system is based on single-strand RNA vectors, which replicate in the cytoplasm and have a low cytotoxicity. Furthermore, transgene expression decreases with cell division, making it one of the most commonly used means of reprogramming. The generation of integration-free iPSCs has also been described using non-viral approaches, such as transfection of DNA plasmids (Okita et al., 2008) or RNA and even the introduction of purified recombinant proteins (Zhou et al., 2009). Often, these approaches have a low efficiency, however. Finally, the use of chemical compounds for reprogramming has been described that do not require the introduction of transcription factors, allowing for stable conditions and outcome. Despite these advances, the mechanisms behind reprogramming remain mostly elusive.

Simultaneously to the advances in reprogramming, culture conditions of iPSCs were also improved. First-generation iPSCs depend on the cocultivation with mouse embryonic fibroblasts (MEFs) and were cultured in serum-supplemented medium. To reduce animal components and thereby making iPSCs more useful for future clinical applications, chemically defined media are currently used, such as Essential 8 (E8) (Chen et al., 2011). Furthermore, xeno-free conditions were developed, which rely on the use of recombinant matrix proteins such as matrigel, geltrex, vitronectin or laminin. Additionally to being xenofree, the improved culture conditions also lead to higher consistency during prolonged cultivation. 


\subsubsection{Cardiac differentiation of hiPSCs}

Currently, three approaches were described to obtain CMs from somatic cells, namely the derivation from iPSCs, the direct transdifferentiation without an intermediate pluripotent status and the combination of both (Kolanowski et al., 2017). During direct transdifferentiation, cardiac transcription factors such as GATA Binding Protein 4 (Gata4), myocyte enhancer factor 2C (Mef2c) and T-box transcription factor-5 (Tbx5) are overexpressed in somatic cells like primary cardiac fibroblasts to induce reprogramming into CMs (Mohamed et al., 2016). This seems especially promising for regenerative medicine since the risk of tumor development is small. Recently chemical-induced CM-like cells were generated by transiently inducing a pluripotent state followed by immediate cardiac differentiation. Importantly, the cells were not genetically modified since only chemical cocktails were used (Fu et al., 2015). The CM yield was increased compared to the direct transdifferentiation, whereas risk for teratoma formation remained small. Induction of a stable pluripotent state and subsequent derivation of $\mathrm{CMs}$ is the most common approach. This approach offers great advantages for basic research, since iPSCs renew themselves and great yields of CMs can be generated for long periods of time.

In early studies CMs were derived from PSCs by using the embryoid body method. Therefor, the PSCs are cultured in suspension in small aggregates, called embryoid bodies (EBs), which causes spontaneous differentiation into cells of all three germ layers. Often, EBs display spontaneous contraction and CMs with ventricular features can be isolated. However, the efficiency of cardiac differentiation is low and the yield of CMs is too small for regenerative applications. For this reason, approaches are developed to directly differentiate PSCs into cardiac cells with high efficiency and purity, by mimicking the embryonic heart development in vitro.

Heart development is a complex process and starts early in embryogenesis during gastrulation. First cardiac precursor cells occur in the heart-forming fields of the anterior lateral plate mesoderm. The formation of the mesoderm and the specification of cardiac precursor cells are tightly regulated by families of signaling molecules such as transforming growth factor type $\beta$ (TGF $\beta$ ), bone morphogenic protein (BMP), fibroblast growth factor (FGF) and WNT. It has been shown that especially canonical WNT signaling inhibits early specification of cardiac cells and that WNT antagonists are located at the heart-forming fields. BMP signaling, on the other hand, induces cardiac specification. During later development, the cardiac precursor cells migrate towards the midline, fuse and thereby form the heart tube which will subsequently loop and form the heart chambers.

In vitro, addition of cytokines that are involved in embryonic heart development such as Activin A, FGF2 or bone morphogenetic protein 4 (BMP4) increases the efficiency of cardiac 
differentiation in EBs (Laflamme et al., 2007; Yang et al., 2008). These principles have also been successfully applied in 2D differentiation systems of iPSCs to obtain CMs (iPSC-CMs). The modulation of the canonical WNT pathway has proven to be particularly effective and can be easily accomplished with small molecules (Gonzalez et al., 2011; Lian et al., 2012; Ren et al., 2011). By activating the canonical WNT pathway via inhibition of GSK3, the cells are directed to a mesodermal fate, which can be observed by upregulation of the protein brachyury. These multipotent mesodermal progenitor cells can be further differentiated into CMs by inactivation of canonical WNT signaling. Yields of up to $90 \%$ iPSC-CMs have been reported using a simple three-step protocol with chemically defined media and recombinant matrix proteins (Burridge et al., 2014). Analytical and medical applications require a high purity of hiPSC-CMs. To accomplish this, the use of antibodies against vascular cell adhesion molecule 1 (VCAM-1) and signal-regulatory protein alpha (SIRPA) has been described for sorting of hiPSC-CMs (Dubois et al., 2011; Uosaki et al., 2011). However, the use of metabolic selection via glucose depletion and lactate supplementation has proven to be highly efficient and is now most commonly used (Tohyama et al., 2013). HiPSC-CMs that are generated in the aforementioned way are a mixture of ventricular-, atrial- and nodal-like cells. They depict distinct electrophysiological properties. Effective protocols to attain homologous populations are still in development (Blazeski et al., 2012; Ma et al., 2011). The majority of iPSC-CMs, generated with common protocols, are ventricular-like cells and their production may further be facilitated by cultivation at normoxia $\left(5 \% \mathrm{O}_{2}\right)$ (Blazeski et al., 2012; Weng et al., 2014). A bigger yield of atrial- and nodal-like cells may be achieved by manipulation of BMP signaling or retinoic acid application at the right differentiation stages (Devalla et al., 2015; Protze et al., 2017). By now, standardized hESC-CMs and hiPSC-CMs are commercially available, for example iCELL cardiomyocytes (Cellular Dynamics) and Cor.4U cardiomyocytes (Axio Genesis). These cells enable quick analyses of hPSC-CMs without previous cultivation and differentiation of hPSCs.

HiPSC-CMs and hESC-CMs, like many other PSC derived cell types, depict an immature phenotype. This may be a result of the rejuvenation that takes place during reprogramming of somatic into pluripotent cells (Mora et al., 2017). In vivo, maturation of CMs is accompanied by changes in the transcriptome such as increased expression of the calcium handling proteins RYR2, SERCA and NCX or subtype-specific proteins like Hairy/enhancer-ofsplit related with YRPW motif protein 2 (Hey2) and ventricular myosin regulatory light chain 2 (Mlc2v) or Hey1 and Mlc2a (atrial). Also, the isoform ratio of certain proteins like titin N2BA and $\mathrm{N} 2 \mathrm{~B}$ changes. Expression of myosin heavy chain isoform 6 (MYH6) decreases during maturation in human CMs whereas MYH7 is expressed in a similar level (Yang et al., 2014a). While most genes are expressed in iPSC-CMs, the expression levels often differ when compared to adult CMs. This also leads to structural and electrophysiological differences. 
Adult CMs undergo hypertrophic growth after birth and depict a typical rod shape, whereas iPSC-CMs are smaller and often circular or multi-angled. Furthermore, adult CMs have a higher organization of the sarcomeric cytoskeleton and the SR and possess specialized structures like T-tubules in the membrane. As a result on the functional level, iPSC-CMs depict a slower conduction velocity across cells and weaker force of contraction. Different approaches have been followed to promote maturation of hiPSC with mild success, including chemical, physical tissue engineering or prolonged cultivation. In conclusion, hiPSC-CMs currently resemble embryonic to neonatal CMs.

\subsubsection{Cardiac applications of hiPSCs}

IPSCs are a powerful tool to study developmental processes as well as mechanisms of reprogramming. However, the greatest potential of the iPSC technology lies in medical applications such as regenerative medicine, disease modeling and patient-specific drug screening (Figure 4). Contrary to hESCs, the use of hiPSCs does not cause ethical problems because the destruction of embryos is not involved. Importantly, hiPSCs retain the donorspecific genetic background, thereby making them disease- and patient-specific (ps-hiPSCs). This feature is a major advantage of hiPSCs over hESCs and opens new possibilities which could not be achieved with hESCs.

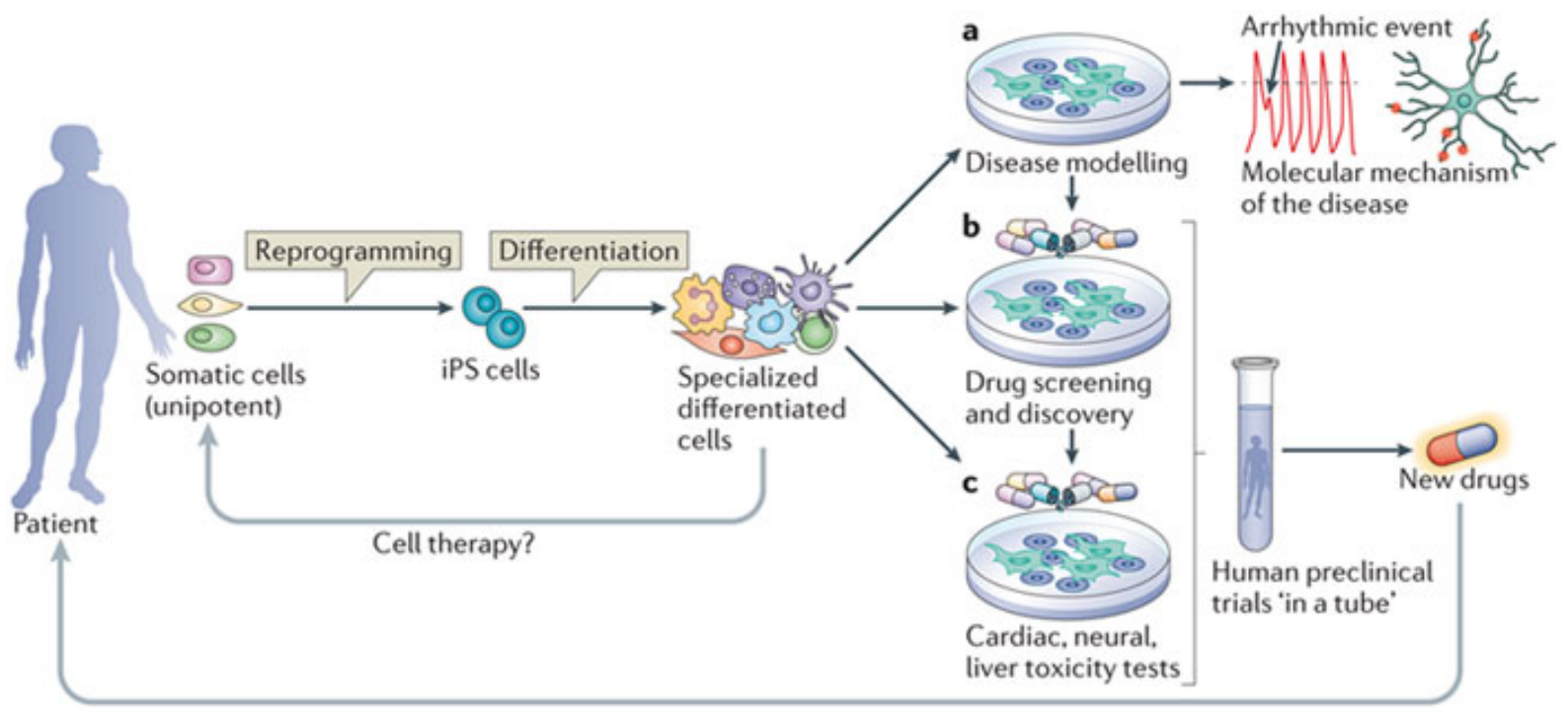

Nature Reviews | Molecular Cell Biology

Figure 4: Applications of ps-hiPSCs. Somatic cells can be isolated from a patient, reprogrammed into iPSCs and derived into specialized differentiated cells such as iPSC-CMs. These cells can be used for disease modeling, drug screening or regenerative medicine. From Bellin et al., 2012. 


\subsubsection{Regenerative medicine}

While regenerative medicine is influenced by and overlaps with many other medical fields and is thereby hard to define, according to Manson and Dunnill it "replaces or regenerates human cells, tissue or organs, to restore or establish normal function" (Mason and Dunnill, 2008). Immune rejection is a major problem in organ transplantation and cell therapy. By transplanting hiPSC-derived differentiated cells or tissue grafts, the patient-specificity of hiPSCs might be used to circumvent this hurdle. On the other hand, the use of hiPSCs in regenerative medicine also involves risks, as residual undifferentiated hiPSCs may form teratoma. Advances have been made to detect these cells (Choudhary and Whiting, 2016; Kuroda et al., 2012) or to eliminate them by high differentiation efficiency and purifying differentiated cells (Hattori et al., 2010; Tohyama et al., 2016). Xenotransplantation experiments with hiPSC-derived cells have already been performed in the fields of Parkinson's disease, spinal cord injury, corneal disease, liver dysfunction and platelet transfusion-refractory. In 2013, the first transplantation in a human was performed with hiPSC-derived retinal-pigmented epithelium (Aoi, 2016).

Regenerative medicine is of special interest for the cardiovascular field, since cardiovascular disease is globally the leading cause of death. However, medical treatment of severe diseases such as heart failure is often limited to the improvement of symptoms or prevention of disease progression. One reason for this is the deficiency of the heart to regenerate muscle tissue after injury. Furthermore, human CMs are hard to acquire and cannot be cultured or expanded in an amount necessary for transplantation or disease research. HiPSCs are a promising source for patient-specific CMs. Research of different approaches using hiPSCs and hESCs has been reported ranging from transplantation of single PSC-CMs, over transplantation of in vitro engineered patches, sheets or tissues, to even the in vitro generation of entire hearts by repopulation of extracellular matrix scaffolds (Anderson et al., 2017). Transplantations of single hPSC-CMs into animal models have often been ineffective in regenerating injured myocardium due to low survival and integration rates of transplanted PSC-CMs (Caspi et al., 2007; van Laake et al., 2007; Laflamme et al., 2007). However, the successful transplantation of hPSC-CMs has been described in a monkey model (Chong et al., 2014). Engineered grafts showed a better survival and improved cardiac function in small animal models and also a pig model. In 2015, hESC-derived cardiac progenitors were transplanted for the first time in a human (Menasché et al., 2015a, 2015b).

\subsubsection{Disease modeling and drug screening}

Disease modeling is another application of hiPSCs and is based on the principle to recapitulate a disease in vitro by differentiating ps-hiPSCs into the affected cell type. These cells can subsequently be used to study the development, progression and mechanisms of 
the disease in a cell type-specific way. Furthermore, a hiPSC disease model may be used to efficiently screen for and develop new drugs, thereby contributing to a patient-specific therapy. Traditionally, human diseases are modeled by using various animals in which the disease phenotype is mimicked by genetic alterations or other means. While animal models played a crucial part in the understanding of numerous diseases, they have several shortcomings, which often cause results to not be reproducible in humans. Modeling diseases with hiPSCs provides distinct advantages. Although the genome of higher animal models and humans is often very similar, crucial differences in development and physiology still exist. For example, the electrophysiology of mouse and human CMs differs with mice action potentials being shorter. Furthermore, mice CMs beat faster and are more often binucleated than human CMs. In vivo, disease phenotypes may result from distinct pathomechanisms. Compensatory effects between cell types can be observed, which makes identification of separate pathomechanisms difficult. Contrary to animal models, ps-hiPSCs possess the disease-specific genetic background, which may contribute to the identification of disease-associated mutations or SNPs. Combination of ps-hiPSCs with the recent clustered regularly interspaced short palindromic repeats (CRISPR)/CRISPR Associated-9 (Cas9) technology creates even further possibilities for disease modeling since isogenic cell lines may be produced with only a single altered basepair. It should also be mentioned that the use of hiPSCs may contribute to the reduction and replacement of animals in scientific research. Various hiPSCs disease models have already been generated in different fields, which recapitulate disease-specific abnormalities in vitro and contributed to the finding of new pathomechanisms.

Long QT syndrome, which can be separated into three forms, is one of the first cardiac arrhythmia diseases that has been modeled with ps-hiPSCs. It is characterized by prolongation of the depolarization of the action potential, is potentially lethal and is caused by mutations in KCNQ1 (potassium voltage-gated channel subfamily KQT member 1), KCNH2 (potassium voltage-gated channel subfamily $\mathrm{H}$ member 2 ) or SCN5A (sodium voltage-gated channel alpha subunit 5) coding for potassium and sodium channels (Tester and Ackerman, 2014). Ps-hiPSC-CMs recapitulate disease-associated phenotypes such as prolonged action potential durations, decreased rectifier potassium currents and increased frequency of early afterdepolarizations and triggered activity (Yoshida and Yamanaka, 2017). Another potentially lethal disease characterized by cardiac arrhythmias and modeled with hiPSCs is catecholaminergic polymorphic ventricular tachycardia (CPVT). Mutations in either RYR2 or CASQ2 cause CPVT. Ps-iPSC-CMs show an increase of intracellular calcium concentration and calcium spark frequency upon stimulation. Importantly, the beneficial effects of the drugs dantrolene, thapsigargin, S107, propranolol and flecanide were validated in these CPVT models (Jung et al., 2012; Sasaki et al., 2016; Yoshida and Yamanaka, 2017). These findings 
demonstrate the potential of ps-hiPSC-CMs to model cardiac arrhythmia and their usefulness in drug screening. Despite cardiac arrhythmias, cardiomyopathies have also been modeled such as the LEOPARD syndrome, which results from mutations in the PTPN11 (tyrosineprotein phosphatase non-receptor type 11) gene. Ps-iPSC-CMs showed phenotypes associated with hypertrophy such as increased size and nuclear translocation of nuclear factor of activated T-cells, cytoplasmic, calcineurin-dependent 4 (NFATC4) (Carvajal-Vergara et al., 2010). Phenotypes regarding the sarcomeric integrity, contractility, calcium handling, nuclear senescence, $\beta$-adrenergic signaling and apoptosis were recapitulated in ps-hiPSCCMs-based models of hypertrophic cardiomyopathy, dilated cardiomyopathy, lamin A/C (LMNA)-related cardiomyopathy and takotsubo syndrome (Borchert et al., 2017; StreckfussBömeke et al., 2017; Yoshida and Yamanaka, 2017). In conclusion, despite the immature state of hiPSC-CMs, important cardiac diseases could be modeled and a broad range of phenotypes were analyzed covering various important CM-specific mechanisms.

\subsection{Modeling ACT using PSC-CMs}

Since robust differentiation protocols became available, several groups analyzed the effects of DOX and other chemotherapeutic agents on hPSC-CMs. Most of them used commercially available hPSC-CMs, which are not ACT patient-specific, to test applicability. Upon DOX treatment, hiPSC-CMs depicted morphological changes, increased beating frequency, arrhythmic beating, cytotoxicity, gene expression changes, apoptosis, increased amount of ROS and reduced contractility (Burridge et al., 2016; Chaudhari et al., 2015, 2016, 2017; Eldridge et al., 2014; Grimm et al., 2015; Zhao and Zhang, 2017). These data demonstrate that hiPSC-CMs recapitulate key features of DOX cardiotoxicity and highlight their potential for modeling ACT. Similar data has also been reported in studies with hESC-CM (Holmgren et al., 2015; Maillet et al., 2016). Importantly, novel biomarkers, pathways and pathomechanisms have been suggested in several hiPSC-CM based studies. Using the same DOX application protocol in three separate studies, Chaudhari et al. showed the DOX-induced deregulation of 35 genes, 14 microRNAs and pyruvate, acetate and formate signatures (Chaudhari et al., 2015, 2016, 2017). The authors propose the clinical use of these changes as early biomarkers of DOX-induced cardiotoxicity. Investigating signaling and cellular response pathways in hiPSC-CMs, NRG/ErbB signaling was validated to be involved in DOX-induced cardiotoxicity (Eldridge et al., 2014). When NRG/ErbB signaling was activated, the DOXinduced damage of iPSC-CMs was reduced, whereas inhibition of ErbB caused the cells to be more sensitive towards DOX. Recently, a novel pathomechanism of ACT has been reported in hiPSC-CMs, namely the DOX-induced gene expression upregulation of the death receptors tumor necrosis factor receptor 1 (TNFR1), Fas-receptor (FasR) and death receptor 4 and 5 (DR4/5) (Zhao and Zhang, 2017). This upregulation would cause an increased susceptibility of 
CMs to TNF-related cytokines and lead to spontaneous apoptosis. This study suggests extrinsic apoptosis as a part of ACT and thereby expands established intrinsic apoptosis models. On the basis of their data, the authors further suggest the use of elevated TNFrelated cytokine serum levels as means to identify ACT risk groups before chemotherapy.

Currently, there is only one report of ps-iPSC-CMs being used to model ACT (Burridge et al., 2016). Here, iPSC-CMs were generated from eight breast cancer patients, four of whom developed ACT after chemotherapy and four of whom did not. Four healthy donors were used as controls. The authors show DOX-induced cytotoxicity, mitochondrial and metabolic impairment, defects in calcium handling, decrease of antioxidants and increase of ROS in iPSC-CMs. Importantly, the phenotypes were more severe in iPSC-CMs from ACT patients compared to non-ACT chemotherapy patients and healthy donors. These data showed for the first time, that ACT can be modeled with ps-iPSC-CMs and suggest, that the genetic background may convey a susceptibility to the development of ACT after chemotherapy (Burridge et al., 2016).

\subsection{Aims of this thesis}

This work aimed to establish a human model system of ACT by using the iPSC technology. Furthermore, functional consequences of ACT-associated SNPs in subunits of the NADPH oxidase were to be investigated in a cardiac background. A human model system of ACT is highly needed because pathomechanisms still remain elusive. Additionally, results that were obtained in animal models are often not reproducible in human studies. A human ACT model could contribute to determine risk factors and biomarkers, discover protective drugs or to develop patient-specific therapies. For this purpose, three patients who suffer from ACT and two controls without cardiac disease after chemotherapy were recruited. The patients had ACT-associated alleles of SNPs in the genes RAC2 and CYBA, whereas the controls did not. The main objectives were:

1. Generation of integration-free ps-iPSCs and subsequent characterization of pluripotency state and developmental potential.

2. Generation of ps-iPSC-CMs with high purity.

3. Analyses of NADPH oxidase subunit expression on mRNA and protein level and of NADPH oxidase activity.

4. Comparison of DOX-induced changes between iPSC-CMs from ACT patients and controls in ROS production, gene expression, calcium handling, apoptosis, sarcomeric integrity and functionality. 
Following hypotheses were made:

1. Untreated iPSC-CMs from ACT patients and controls do not differ in functionality, since no cardiac phenotype was reported in either group before chemotherapy.

2. DOX treatment causes ACT-associated abnormalities in iPSC-CMs.

3. iPSC-CMs from ACT patients react more sensitive to DOX treatment than control iPSCCMs.

4. Differences between iPSC-CMs from ACT patients and controls in DOX-induced phenotypes are associated with SNPs in NADPH oxidase subunits. 


\section{Materials}

\subsection{Chemicals, reagents, basal media and enzymes}

\begin{tabular}{|c|c|}
\hline Name & Provider and product number \\
\hline $0.25 \%$ Trypsin-EDTA & Thermo Fisher Scientific \#25200056 \\
\hline $\begin{array}{l}\text { 4', 6-Diamidino-2-phenylindole dihydrochloride } \\
\text { (DAPI) }\end{array}$ & Sigma-Aldrich \#D9542 \\
\hline Agar & Serva Electrophoresis \#200201 \\
\hline Albumin, human recombinant & Sigma-Aldrich \#A0237/A9731 \\
\hline 6-Aminohexanoic acid & $\begin{array}{l}\text { PanReac AppliChem ITW Reagents } \\
\text { \#A2266,0500 }\end{array}$ \\
\hline Ammonium persulfate (APS) & Roth \#9178 \\
\hline B-27 serum free supplement (50x) & Thermo Fisher Scientific \#17504044 \\
\hline B-27 Supplement, minus insulin & Thermo Fisher Scientific \#A1895601 \\
\hline Boric acid & Sigma-Aldrich \#15663 \\
\hline Bovine albumin fraction V solution (BSA, 7.5\%) & Thermo Fisher Scientific \#15260037 \\
\hline Bromophenol blue sodium salt & Roth \#A512.2 \\
\hline Calcium chloride dihydrate $\left(\mathrm{CaCl}_{2}\right)$ & Roth \#HN04 \\
\hline CHIR99021 & Merck Millipore \#361559 \\
\hline Collagenase IV & Worthington Biochemical \#CLS-4 \\
\hline cOmplete (protease inhibitor cocktail tablets) & Roche \#04693132001 \\
\hline $\begin{array}{l}\text { Di-Sodium hydrogen phosphate dihydrate } \\
\left(\mathrm{Na}_{2} \mathrm{HPO}_{4} \cdot 2 \mathrm{H} 2 \mathrm{O}\right)\end{array}$ & Roth \#T877 \\
\hline Diamide & Santa Cruz \#sc-211289 \\
\hline Dimethyl sulfoxide (DMSO) & Sigma-Aldrich \#D2650 \\
\hline Dithiothreitol (DTT) & Roth \#6908 \\
\hline Dulbecco’s Modified Eagle Medium (DMEM) & Thermo Fisher Scientific \#11960044 \\
\hline DMEM/F-12 & Thermo Fisher Scientific \#31331028 \\
\hline dNTP mix & Bioline \#BIO-39029 \\
\hline Doxorubicin hydrochloride & Sigma-Aldrich \#D1515 \\
\hline $\begin{array}{l}\text { Dulbecco's Phosphate-Buffered Saline (DPBS), } \\
\text { no calcium, no magnesium }\end{array}$ & Thermo Fisher Scientific \#14190144 \\
\hline Essential 8 basal medium & Thermo Fisher Scientific \#A1517001 \\
\hline Ethylenediaminetetraacetic acid (EDTA) & Sigma-Aldrich \#E6758 \\
\hline
\end{tabular}




\begin{tabular}{|c|c|}
\hline Name & Provider and product number \\
\hline Fetal bovine serum (FBS) & Sigma-Aldrich \#F7524 \\
\hline FGF2 & Peprotech \#AF-100-18B \\
\hline Fluo-4/AM, cell permeant & Thermo Fisher Scientific \#F14201 \\
\hline Fluoromount-G & eBioscience \#00-4958-02 \\
\hline Formalin (37 \%) (PFA) & Merck Millipore \#1039991000 \\
\hline Gelatin & Sigma-Aldrich \#48720 \\
\hline Geltrex & Thermo Fisher Scientific \#A1413301 \\
\hline GeneAmp 10X PCR Buffer II \& $\mathrm{MgCl}_{2}$ & Thermo Fisher Scientific \#4379878 \\
\hline GeneRuler 100 bp Plus DNA Ladder & Thermo Fisher Scientific \#0321 \\
\hline Glucose & Sigma-Aldrich \#G8270 \\
\hline Glycerol & Roth \#3783 \\
\hline Glycine & Roth \#3908 \\
\hline GoTaq G2 DNA polymerase & Promega \#M7845 \\
\hline HEPES & Roth \#9105 \\
\hline HEPES sodium salt solution (1 M) & Sigma-Aldrich \#H3662 \\
\hline Hexadimethrine bromide (Polybrene) & Sigma Aldrich \#107689 \\
\hline Hydrochloric acid fuming 37\% (HCl) & Merck Millipore \#100317 \\
\hline IGEPAL CA-630 & Sigma-Aldrich \#I3021 \\
\hline IGF1 & Peprotech \#AF-100-11 \\
\hline $\begin{array}{l}\text { Iscove's modified Dulbecco's medium with } \\
\text { GlutaMax (IMDM) }\end{array}$ & Thermo Fisher Scientific \#31980022 \\
\hline Isoprenaline hydrochloride (ISO) & Sigma-Aldrich \#II5627 \\
\hline Isopropanol & Merck Millipore \#109634 \\
\hline IWP2 & Merck Millipore \#681671 \\
\hline Kanamycin sulfate & Roth \#T832 \\
\hline Knockout serum replacement & Thermo Fisher Scientific \#10828028 \\
\hline L-ascorbic acid 2-phosphate & Sigma-Aldrich \#A8960 \\
\hline L-glutamine (200 mM, 100x) & Thermo Fisher Scientific \#25030024 \\
\hline LB bouillon (Miller) & Merck \#1102850500 \\
\hline Magnesium chloride $\left(\mathrm{MgCl}_{2}\right)$ & Sigma-Aldrich \#M8266 \\
\hline Methanol & Merck Millipore \#106009 \\
\hline Midori Green Advance & Biozym \#617004 \\
\hline Mitomycin C (MMC) & Serva Electrophoresis \#29805.02 \\
\hline
\end{tabular}




\begin{tabular}{|c|c|}
\hline Name & Provider and product number \\
\hline Monothioglycerol (MTG) & Sigma-Aldrich \#M6145-25ML \\
\hline MuLV reverse transcriptase (50 U/ $\mu \mathrm{l})$ & Thermo Fisher Scientific \#N808-0018 \\
\hline Non-essential amino acids (NEAA, 100x) & Thermo Fisher Scientific \#11140035 \\
\hline Nonfat dry milk & Roth \#T145 \\
\hline Nuclease-free water & Thermo Fisher Scientific \#AM9932 \\
\hline Oligo d(T)16 (50 $\mu \mathrm{M})$ & Thermo Fisher Scientific \#N808-0128 \\
\hline Opti-MEM & Thermo Fischer Scientific \#4309155 \\
\hline Paraformaldehyde (PFA) & Sigma-Aldrich \#158127 \\
\hline Penicillin-streptomycin solution (P/S) (100x) & Thermo Fisher Scientific \#15140122 \\
\hline PeqGold protein marker V & Peqlab \#27-2210 \\
\hline peqGold universal agarose & Peqlab \#35-1020 \\
\hline pH neutralized medical grade bovine collagen & LLC Collagen Solutions \\
\hline PhosStop (phosphatase inhibitor cocktail tablets) & Roche \#04906837001 \\
\hline Pluronic F-127 & Thermo Fisher Scientific \#P3000MP \\
\hline Polyfect & Qiagen \#301105 \\
\hline Potassium chloride (KCl) & Sigma-Aldrich \#P9541 \\
\hline $\begin{array}{l}\text { Recombinant human basic fibroblast growth } \\
\text { factor (hbFGF) }\end{array}$ & PeproTech \#100-18B \\
\hline 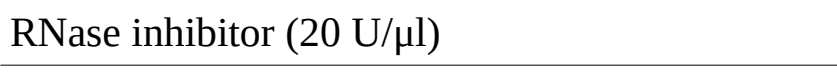 & Thermo Fisher Scientific \#N808-0119 \\
\hline Rotiphorese gel 30 & Roth \#3029 \\
\hline RPMI 1640 with HEPES with GlutaMax & Thermo Fisher Scientific \#72400021 \\
\hline RPMI 1640, no Glucose & Thermo Fisher Scientific \#11879020 \\
\hline Sodium chloride $(\mathrm{NaCl})$ & Roth \#9265.1 \\
\hline Sodium DL-lactate solution 60\% (w/w) & Sigma-Aldrich \#L4263 \\
\hline Sodium dodecyl sulfate (SDS) & Roth \#2326 \\
\hline Sodium fluoride $(\mathrm{NaF})$ & Roth \#P756 \\
\hline SYBR Green PCR Master Mix & Thermo Fischer Scientific \#4309155 \\
\hline Tetramethylethylenediamine (TEMED) & Roth \#2367 \\
\hline TGF-b1 & Peprotech \#AF-100-21C \\
\hline Thiazovivin (TZV) & Merck Millipore \# 420220 \\
\hline Tricin & $\begin{array}{l}\text { PanReac AppliChem ITW Reagents } \\
\text { \#A1085,0100 }\end{array}$ \\
\hline Tris & Roth \#5429 \\
\hline Triton $\mathrm{X}-100$ & Sigma-Aldrich \#3051.3 \\
\hline
\end{tabular}




\begin{tabular}{l|l} 
Name & Provider and product number \\
\hline \hline TrypLE Express Enzyme (1x), no phenol red & Thermo Fisher Scientific \#12604013 \\
\hline Tween 20 & Bio-Rad \#170-6531 \\
\hline VEGF $_{165}$ & Peprotech \#AF-100-20 \\
\hline Versene solution $(0.48 \mathrm{mM}$ EDTA) & Thermo Fisher Scientific \#15040066 \\
\hline$\beta$-Mercaptoethanol $(\beta-\mathrm{ME})$ & Serva Electrophoresis \#28625 \\
\hline
\end{tabular}

\subsection{Commercial kits}

\begin{tabular}{l|l} 
Name & Provider \\
\hline Alkaline phosphatase staining kit & Sigma-Aldrich \#86R-1KT \\
\hline $\begin{array}{l}\text { Amplex Red Hydrogen Peroxide/Peroxidase } \\
\text { Assay Kit }\end{array}$ & Thermo Fisher Scientific \#A22188 \\
\hline APC Annexin V Apoptosis Detection Kit with PI & BioLegend \#640932 \\
\hline CytoTune-iPS 2.0 Sendai reprogramming kit & Thermo Fisher Scientific \#A16517 \\
\hline $\begin{array}{l}\text { Immobilon Western Chemiluminescent HPR } \\
\text { Substrate }\end{array}$ & Merck Millipore \#WBKLS0500 \\
\hline Maxwell 16 cell DNA purification kit & Promega \#AS1020 \\
\hline NucleoBond Xtra Maxi Plus EF Kit & Macherey-Nagel \#740426.10 \\
\hline Pierce BCA protein assay kit & Thermo Fisher Scientific \#23225 \\
\hline QIAquick gel extraction kit & Qiagen \#28706 \\
\hline SV total RNA isolation system & Promega \#Z3105 \\
\hline
\end{tabular}

\subsection{Buffers and solutions for molecular biological analyses}

\begin{tabular}{|c|c|c|}
\hline Name & \multicolumn{2}{|c|}{ Composition and specifications } \\
\hline \multirow[t]{2}{*}{ Triton-X-100 (0.1%) } & & DPBS \\
\hline & $0.1 \%$ & Triton-X-100 \\
\hline \multirow[t]{3}{*}{ Agarose-Gel (1 - 1.5\%) } & $1 \mathrm{x}$ & TB-buffer \\
\hline & $1-1.5 \%$ & Agarose \\
\hline & $\begin{array}{l}0.07 \\
\mu \mathrm{L} / \mathrm{mL}\end{array}$ & Midori Green Advance \\
\hline \multirow[t]{3}{*}{ TB-buffer (5x) } & & Deionized $\mathrm{H}_{2} \mathrm{O}$ \\
\hline & 54 g/L & Tris \\
\hline & $27.5 \mathrm{~g} / \mathrm{L}$ & Boric acid \\
\hline
\end{tabular}




\begin{tabular}{|c|c|c|}
\hline Name & \multicolumn{2}{|c|}{ Composition and specifications } \\
\hline \multirow[t]{4}{*}{ DAPI } & \multirow{4}{*}{$\begin{array}{l}2 \\
\mathrm{mg} / \mathrm{mL}\end{array}$} & Deionized $\mathrm{H}_{2} \mathrm{O}$ \\
\hline & & DAPI \\
\hline & & \\
\hline & & $\begin{array}{l}\text { For Working solution diluted 1:5000 in } \\
1 \% \text { BSA in DPBS }\end{array}$ \\
\hline \multirow[t]{3}{*}{ PFA (4\%) } & \multirow{3}{*}{$4 \%$} & DPBS \\
\hline & & PFA \\
\hline & & Store at $-20^{\circ} \mathrm{C}$ \\
\hline \multirow[t]{3}{*}{ Tris-HCl, pH 7.4 (2 M) } & \multirow{3}{*}{$2 \mathrm{M}$} & Deionized $\mathrm{H}_{2} \mathrm{O}$ \\
\hline & & Tris \\
\hline & & $\begin{array}{l}\mathrm{pH} \text { adjusted to } 7.4 \text {; stored at room } \\
\text { temperature }\end{array}$ \\
\hline \multirow[t]{10}{*}{ Protein lysis buffer } & \multirow{10}{*}{$\begin{array}{l}20 \mathrm{mM} \\
200 \mathrm{mM} \\
20 \mathrm{mM} \\
1 \% \\
1 \mathrm{mM} \\
1 \mathrm{mM} \\
1 \text { Tablet } \\
/ 10 \mathrm{~mL} \\
1 \text { Tablet } \\
\text { / } 20 \mathrm{~mL}\end{array}$} & Deionized $\mathrm{H}_{2} \mathrm{O}$ \\
\hline & & Tris-HCl, pH 7.4 \\
\hline & & $\mathrm{NaCl}$ \\
\hline & & $\mathrm{NaF}$ \\
\hline & & Igepal \\
\hline & & $\mathrm{Na}_{3} \mathrm{VO}_{4}$ \\
\hline & & $\mathrm{DTT}$ \\
\hline & & PhosStop \\
\hline & & Complete \\
\hline & & Freshly prepared \\
\hline \multirow[t]{4}{*}{ TBST-Buffer } & \multirow{4}{*}{$\begin{array}{l}20 \mathrm{mM} \\
150 \mathrm{mM} \\
0.1 \%\end{array}$} & Deionized $\mathrm{H}_{2} \mathrm{O}$ \\
\hline & & Tris \\
\hline & & $\mathrm{NaCl}$ \\
\hline & & Tween 20 \\
\hline \multirow[t]{4}{*}{ Running buffer } & \multirow{4}{*}{$\begin{array}{l}25 \mathrm{mM} \\
192 \mathrm{mM} \\
0.1 \%\end{array}$} & Deionized $\mathrm{H}_{2} \mathrm{O}$ \\
\hline & & Tris \\
\hline & & Glycine \\
\hline & & SDS \\
\hline
\end{tabular}




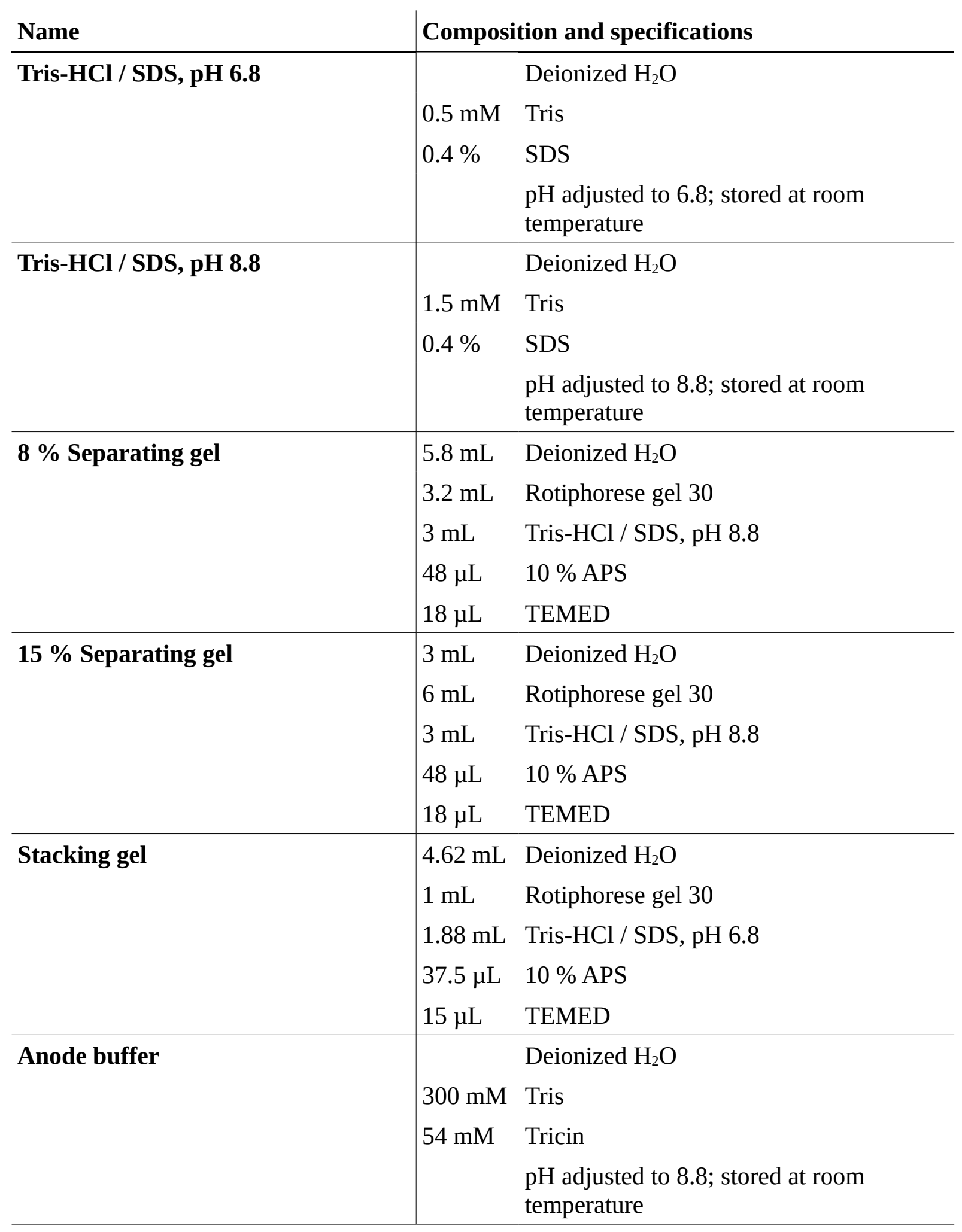




\begin{tabular}{|c|c|c|}
\hline Name & \multicolumn{2}{|c|}{ Composition and specifications } \\
\hline \multirow[t]{4}{*}{ Cathode buffer } & & Deionized $\mathrm{H}_{2} \mathrm{O}$ \\
\hline & $30 \mathrm{mM}$ & Tris \\
\hline & $300 \mathrm{mM}$ & 6-Aminohexanoic acid \\
\hline & & $\begin{array}{l}\mathrm{pH} \text { adjusted to } 8.7 \text {; stored at room } \\
\text { temperature }\end{array}$ \\
\hline \multirow[t]{8}{*}{ SDS sample buffer (5x) } & & Deionized $\mathrm{H}_{2} \mathrm{O}$ \\
\hline & $313 \mathrm{mM}$ & Tris-HCl pH 6.8 \\
\hline & $10 \%$ & SDS \\
\hline & $0.05 \%$ & Bromophenol blue \\
\hline & $50 \%$ & Glycerol \\
\hline & $5 \mathrm{mM}$ & EDTA \\
\hline & $150 \mathrm{mM}$ & DTT \\
\hline & & Stored at $-20^{\circ} \mathrm{C}$ \\
\hline \multirow[t]{2}{*}{ DTT (110 mM) } & & Tyrode’s solution \\
\hline & $110 \mathrm{mM}$ & DTT \\
\hline \multirow[t]{2}{*}{ Diamide (5.5 mM) } & & Tyrode’s solution \\
\hline & $5.5 \mathrm{mM}$ & Diamide \\
\hline \multirow[t]{8}{*}{ Tyrode's solution } & & Deionized $\mathrm{H}_{2} \mathrm{O}$ \\
\hline & $150 \mathrm{mM}$ & $\mathrm{NaCl}$ \\
\hline & $5.4 \mathrm{mM}$ & $\mathrm{KCl}$ \\
\hline & $1.8 \mathrm{mM}$ & $\mathrm{CaCl}_{2}$ \\
\hline & $1 \mathrm{mM}$ & $\mathrm{MgCl}_{2}$ \\
\hline & $10 \mathrm{mM}$ & HEPES \\
\hline & $10 \mathrm{mM}$ & Glucose \\
\hline & & $\mathrm{pH}$ adjusted to 7.4 ; stored at $4^{\circ} \mathrm{C}$ \\
\hline \multirow[t]{3}{*}{ Amplex Red working solution 1} & & DPBS \\
\hline & $50 \mathrm{nM}$ & Amplex Red \\
\hline & $\begin{array}{l}50 \\
\mathrm{mU} / \mathrm{mL}\end{array}$ & Horseradish peroxidase \\
\hline \multirow[t]{3}{*}{ Amplex Red working solution 2} & & DPBS \\
\hline & $100 \mathrm{nM}$ & Amplex Red \\
\hline & $\begin{array}{l}100 \\
\mathrm{mU} / \mathrm{mL}\end{array}$ & Horseradish peroxidase \\
\hline
\end{tabular}




\subsection{Media supplements, factors and solutions for cell culture}

\begin{tabular}{|c|c|c|}
\hline Name & \multicolumn{2}{|c|}{ Composition and specifications } \\
\hline \multirow[t]{3}{*}{ Gelatin (1 \%) } & & Deionized $\mathrm{H}_{2} \mathrm{O}$ \\
\hline & $1 \%$ & Gelatin \\
\hline & & Autoclaved and stored at $4^{\circ} \mathrm{C}$. \\
\hline \multirow[t]{3}{*}{ EDTA (50x) } & & DPBS \\
\hline & $1 \%$ & EDTA \\
\hline & & $\begin{array}{l}\mathrm{pH} \text { adjusted to } 7 \text {, sterile filtrated and } \\
\text { stored at } 4^{\circ} \mathrm{C}\end{array}$ \\
\hline \multirow[t]{3}{*}{ Trypsin $(0.2 \%)$} & & DPBS \\
\hline & $0.2 \%$ & Trypsin \\
\hline & & Sterile filtrated and stored at $4^{\circ} \mathrm{C}$ \\
\hline \multirow[t]{4}{*}{ Trypsin/EDTA (T/E; $0.1 \%)$} & $1 \mathrm{~mL}$ & 50x EDTA \\
\hline & $50 \mathrm{~mL}$ & $0.2 \%$ Trypsin \\
\hline & $49 \mathrm{~mL}$ & DPBS \\
\hline & & Stored at $4^{\circ} \mathrm{C}$ \\
\hline \multirow[t]{3}{*}{ MTG (150 mM) } & & IMDM \\
\hline & $150 \mathrm{mM}$ & MTG \\
\hline & & Sterile filtrated and freshly prepared \\
\hline \multirow[t]{3}{*}{$\beta$-ME (10 mM) } & & DPBS \\
\hline & $10 \mathrm{mM}$ & $\beta-\mathrm{ME}$ \\
\hline & & Sterile filtrated and stored at $4^{\circ} \mathrm{C}$ \\
\hline \multirow[t]{3}{*}{ BSA (1\%) } & $6.5 \mathrm{~mL}$ & DPBS \\
\hline & $1 \mathrm{~mL}$ & 7.5\% BSA solution \\
\hline & & Stored at $4^{\circ} \mathrm{C}$ \\
\hline \multirow[t]{3}{*}{ CHIR (12 mM) } & $894 \mu \mathrm{L}$ & DMSO \\
\hline & $5 \mathrm{mg}$ & CHIR99029 \\
\hline & & Stored at $-20^{\circ} \mathrm{C}$ \\
\hline \multirow[t]{3}{*}{ Collagenase IV (2000 U/mL) } & $1 \mathrm{~mL}$ & DMEM/F12 \\
\hline & $2000 \mathrm{U}$ & Collagenase IV \\
\hline & & $\begin{array}{l}\text { Sterile filtrated and stored at }-20^{\circ} \mathrm{C} \text {; } \\
\text { working solution } 200 \mathrm{U} / \mathrm{mL}\end{array}$ \\
\hline
\end{tabular}




\begin{tabular}{|c|c|c|}
\hline & Compos & tion and specifications \\
\hline Polybrene (1 mg/mL) & $\begin{array}{l}1 \mathrm{~mL} \\
1 \mathrm{mg}\end{array}$ & $\begin{array}{l}\text { Deionized } \mathrm{H}_{2} \mathrm{O} \\
\text { Polystyrene } \\
\text { Sterile filtrated and stored at } 4^{\circ} \mathrm{C}\end{array}$ \\
\hline HbFGF (100 ng/ $\mu L)$ & $\begin{array}{l}1 \mathrm{~mL} \\
100 \mathrm{mg}\end{array}$ & $\begin{array}{l}5 \mathrm{mM} \text { Tris } \\
\mathrm{hbFGF} \\
\text { Stored at }-20^{\circ} \mathrm{C} \text {. For a working solution of } \\
5 \mathrm{ng} / \mu \mathrm{L} \text { the stock solution was diluted } \\
1: 20 \text { in } 0.1 \% \text { BSA and stored at } 4^{\circ} \mathrm{C} \text { not } \\
\text { longer than two weeks. }\end{array}$ \\
\hline ISO (200 mM) & $\begin{array}{l}1 \mathrm{~mL} \\
49.44 \\
\mathrm{mg}\end{array}$ & $\begin{array}{l}\text { Deionized } \mathrm{H}_{2} \mathrm{O} \\
\text { ISO }\end{array}$ \\
\hline IWP2 (5mM) & $\begin{array}{l}4.28 \mathrm{~mL} \\
10 \mathrm{mg}\end{array}$ & $\begin{array}{l}\text { DMSO } \\
\text { IWP2 } \\
\text { Dilution at } 37^{\circ} \mathrm{C} \text { for } 10 \mathrm{~min} \text {. Stored at } \\
-20^{\circ} \mathrm{C}\end{array}$ \\
\hline Mitomycin C (200 $\mu \mathrm{g} / \mathrm{mL})$ & $\begin{array}{l}1 \mathrm{~mL} \\
0.2 \mathrm{mg}\end{array}$ & $\begin{array}{l}\text { DPBS } \\
\text { Mitomycin } \mathrm{C} \\
\text { Stored at }-20^{\circ} \mathrm{C}\end{array}$ \\
\hline TZV (2 mM) & $\begin{array}{l}6.8 \mathrm{~mL} \\
10 \mathrm{mg}\end{array}$ & $\begin{array}{l}\text { DMSO } \\
\text { TZV } \\
\text { Stored at }-20^{\circ} \mathrm{C}\end{array}$ \\
\hline DOX (5mM) & $5 \mathrm{mM}$ & $\begin{array}{l}\text { Deionized } \mathrm{H}_{2} \mathrm{O} \\
\text { DOX } \\
\text { Stored at }-20^{\circ} \mathrm{C}\end{array}$ \\
\hline Lactate/HEPES (1 M) & $\begin{array}{l}3 \mathrm{~mL} \\
18 \mathrm{~mL}\end{array}$ & $\begin{array}{l}\text { Sodium DL-lactate solution } 60 \%(\mathrm{w} / \mathrm{w}) \\
1 \mathrm{M} \text { HEPES sodium salt solution } \\
\text { Stored at }-20^{\circ} \mathrm{C}\end{array}$ \\
\hline
\end{tabular}




\subsection{Cell culture media}

\begin{tabular}{|c|c|c|}
\hline Name & \multicolumn{2}{|c|}{ Composition and specifications } \\
\hline \multirow{5}{*}{$\begin{array}{l}\text { Human Embryonic Stem Cell } \\
\text { Medium (hES-medium) }\end{array}$} & & DMEM/F12 with GlutaMAX \\
\hline & $20 \%$ & Knock-Out Serum Replacement \\
\hline & $1 \mathrm{x}$ & NEAA \\
\hline & $1 \mathrm{x}$ & $\beta-\mathrm{ME}$ \\
\hline & $10 \mathrm{ng} / \mathrm{mL}$ & hbFGF \\
\hline \multirow[t]{4}{*}{ Iscove medium } & & IMDM with GlutaMAX \\
\hline & $20 \%$ & Heat-inactivated FBS \\
\hline & $1 \mathrm{x}$ & NEAA \\
\hline & $450 \mu \mathrm{M}$ & MTG \\
\hline \multirow[t]{3}{*}{ Freezing medium 1} & & DMEM \\
\hline & $20 \%$ & Heat-inactivated FBS \\
\hline & $8 \%$ & DMSO \\
\hline \multirow{6}{*}{$\begin{array}{l}\text { Human Fibroblast Medium (HFB- } \\
\text { medium) }\end{array}$} & & DMEM \\
\hline & $10 \%$ & Heat-inactivated FBS \\
\hline & $1 \mathrm{x}$ & L-Glutamine \\
\hline & $1 \mathrm{x}$ & NEAA \\
\hline & $1 \mathrm{x}$ & $\beta-\mathrm{ME}$ \\
\hline & $10 \mathrm{ng} / \mathrm{mL}$ & hbFGF \\
\hline \multirow[t]{2}{*}{ Essential 8 medium (E8 medium) } & & Essential 8 basal medium \\
\hline & $1 \mathrm{x}$ & Essential 8 Supplement \\
\hline \multirow[t]{4}{*}{ Differentiation medium } & & RPMI 1640 with HEPES and GlutaMAX \\
\hline & $\begin{array}{l}500 \\
\mu \mathrm{g} / \mathrm{mL}\end{array}$ & Albumin, human recombinant \\
\hline & $\begin{array}{l}100 \\
\mu \mathrm{g} / \mathrm{mL}\end{array}$ & L-ascorbic acid 2-phosphate \\
\hline & & Sterile filtrated \\
\hline \multirow[t]{2}{*}{ Cardiac culture medium } & & RPMI 1640 with HEPES and GlutaMAX \\
\hline & $1 \mathrm{x}$ & B27 Serum-free Supplement \\
\hline \multirow[t]{3}{*}{ Freezing medium 2} & & E8 medium \\
\hline & $20 \%$ & DMSO \\
\hline & $4 \mu \mathrm{M}$ & $\mathrm{TZV}$ \\
\hline
\end{tabular}




\begin{tabular}{|c|c|c|}
\hline Name & \multicolumn{2}{|c|}{ Composition and specifications } \\
\hline \multirow[t]{3}{*}{ Digestion medium } & & Cardiac culture medium \\
\hline & $20 \%$ & Heat-inactivated FBS \\
\hline & $2 \mu \mathrm{M}$ & $\mathrm{TZV}$ \\
\hline \multirow{3}{*}{$\begin{array}{l}\text { Mouse embryonic fibroblast- } \\
\text { medium (MEF-medium) }\end{array}$} & & DMEM \\
\hline & $15 \%$ & Heat-inactivated FBS \\
\hline & $1 \mathrm{x}$ & L-Glutamine \\
\hline \multirow[t]{5}{*}{ Selection medium } & & RPMI 1640, no glucose \\
\hline & $4 \mathrm{mM}$ & Lactate/HEPES \\
\hline & $\begin{array}{l}500 \\
\mu \mathrm{g} / \mathrm{mL}\end{array}$ & Albumin, human recombinant \\
\hline & $\begin{array}{l}100 \\
\mu g / m L\end{array}$ & L-Ascorbic acid 2-phosphate \\
\hline & & Sterile filtrated \\
\hline \multirow[t]{3}{*}{ HEK transfection-medium } & & DMEM \\
\hline & $0.5 \%$ & Heat-inactivated FBS \\
\hline & $1 \mathrm{x}$ & L-Glutamine \\
\hline \multirow[t]{3}{*}{ HEK-medium } & & DMEM \\
\hline & $10 \%$ & Heat-inactivated FBS \\
\hline & $1 \mathrm{x}$ & L-Glutamine \\
\hline \multirow[t]{5}{*}{ EHM-reconstitution mixture } & $2 x$ & RPMI \\
\hline & $8 \%$ & B27 without insulin \\
\hline & $\begin{array}{l}0.4 \\
\mathrm{mg} / \mathrm{EHM}\end{array}$ & $\begin{array}{l}\mathrm{pH} \text { neutralized medical grade bovine } \\
\text { collagen }\end{array}$ \\
\hline & $200 \mathrm{U} / \mathrm{ml}$ & Penicillin \\
\hline & $\begin{array}{l}200 \\
\mu g / \mathrm{ml}\end{array}$ & Streptomycin \\
\hline
\end{tabular}




\begin{tabular}{|c|c|c|}
\hline Name & \multicolumn{2}{|c|}{ Composition and specifications } \\
\hline \multirow[t]{11}{*}{ EHM-culture medium } & & Iscove medium \\
\hline & $4 \%$ & B27 without insulin \\
\hline & $1 \%$ & NEAA \\
\hline & $2 \mathrm{mM}$ & Glutamine \\
\hline & $300 \mu \mathrm{M}$ & Ascorbic acid \\
\hline & $\begin{array}{l}100 \\
\mathrm{ng} / \mathrm{mL}\end{array}$ & IGF1 \\
\hline & $10 \mathrm{ng} / \mathrm{mL}$ & FGF2 \\
\hline & $5 \mathrm{ng} / \mathrm{mL}$ & VEGF $_{165}$ \\
\hline & $5 \mathrm{ng} / \mathrm{mL}$ & TGF-b1 \\
\hline & $\begin{array}{l}100 \\
\mathrm{U} / \mathrm{mL}\end{array}$ & Penicillin \\
\hline & $\begin{array}{l}100 \\
\mu \mathrm{g} / \mathrm{mL}\end{array}$ & Streptomycin \\
\hline
\end{tabular}

\subsection{Cell lines}

\begin{tabular}{l|l|l|l} 
Cell type & $\begin{array}{l}\text { Internal } \\
\text { identification }\end{array}$ & Donor & Specifications \\
\hline $\begin{array}{l}\text { human } \\
\text { primary } \\
\text { dermal } \\
\text { fibroblasts }\end{array}$ & ACT1 & Patient 1 & $\begin{array}{l}\text { Derived from skin biopsies of a 69-year-old male } \\
\text { patient with ACT and alleles C/T of rs4673 and } \\
\text { alleles A/A of rs13058338. }\end{array}$ \\
\cline { 2 - 4 } & ACT2 & Patient 2 & $\begin{array}{l}\text { Derived from skin biopsies of a 71-year-old male } \\
\text { patient with ACT and alleles C/T of rs4673 and } \\
\text { alleles T/A of rs13058338. }\end{array}$ \\
\cline { 2 - 4 } & ACT3 & Patient 3 & $\begin{array}{l}\text { Derived from skin biopsies of a 66-year-old female } \\
\text { patient with ACT and alleles C/T of rs4673 and } \\
\text { alleles T/A of rs13058338. }\end{array}$ \\
\cline { 2 - 4 } & ACT.K1 & Control 1 & $\begin{array}{l}\text { Derived from skin biopsies of a 66-year-old male } \\
\text { donor without heart condition after chemotherapy } \\
\text { with alleles C/C of rs4673 and alleles T/T of } \\
\text { rs13058338. }\end{array}$ \\
\cline { 2 - 4 } & ACT.K2 & Control 2 & $\begin{array}{l}\text { Derived from skin biopsies of a 68-year-old male } \\
\text { donor without heart condition after chemotherapy } \\
\text { with alleles C/C of rs4673 and alleles T/T of } \\
\text { rs13058338. }\end{array}$ \\
\hline
\end{tabular}




\begin{tabular}{|c|c|c|c|}
\hline Cell type & $\begin{array}{l}\text { Internal } \\
\text { identification }\end{array}$ & Donor & Specifications \\
\hline \multirow{11}{*}{$\begin{array}{l}\text { hiPSCs } \\
\text { and iPSC- } \\
\text { CMs }\end{array}$} & is.ACT.1.9 & \multirow[t]{2}{*}{ Patient 1} & \multirow{10}{*}{$\begin{array}{l}\text { Generated from respective human primary dermal } \\
\text { fibroblasts with the CytoTune-iPS } 2.0 \\
\text { reprogramming Kit (Thermo Fisher Scientific). }\end{array}$} \\
\hline & is.ACT.1.10 & & \\
\hline & is.ACT.2.11 & \multirow[t]{2}{*}{ Patient 2} & \\
\hline & is.ACT.2.14 & & \\
\hline & is.ACT.3.1 & \multirow[t]{2}{*}{ Patient 3} & \\
\hline & is.ACT.3.11 & & \\
\hline & is.ACT.K1.2 & \multirow[t]{2}{*}{ Control 1} & \\
\hline & is.ACT.K1.8 & & \\
\hline & is.ACT.K2.7 & \multirow[t]{2}{*}{ Control 2} & \\
\hline & is.ACT.K2.9 & & \\
\hline & FB1 & Healthy & $\begin{array}{l}\text { Generated in-house from primary dermal fibroblasts } \\
\text { of a healthy donor (University Medical Center } \\
\text { Göttingen). Characterization and proof of } \\
\text { pluripotency was published earlier (Streckfuss- } \\
\text { Bömeke et al., 2013). }\end{array}$ \\
\hline $\begin{array}{l}\text { Mouse } \\
\text { embryonic } \\
\text { fibroblasts } \\
\text { (MEFs) }\end{array}$ & - & - & $\begin{array}{l}\text { Derived from E14.5 embryos of Naval Medical } \\
\text { Research Institute (NMRI) mice; central animal } \\
\text { facility, University Medical Center, Göttingen. }\end{array}$ \\
\hline $\begin{array}{l}\text { Human } \\
\text { embryonic } \\
\text { kidney } \\
\text { cells } \\
\text { (HEK) }\end{array}$ & HEK-293T & - & $\begin{array}{l}\text { Human embryonic kidney cells transformed with } \\
\text { adenovirus } 5 \text { and SV40 Large T-antigen DNA. }\end{array}$ \\
\hline
\end{tabular}

\subsection{Oligonucleotides}

\begin{tabular}{l|l|l|l|l|l} 
Name & Sequence (5’ $\left.\rightarrow \mathbf{3}^{\prime}\right)$ & Purpose & $\mathbf{b p}$ & $\begin{array}{l}\mathbf{T}_{\mathbf{A}} \\
{\left[{ }^{\circ} \mathbf{C}\right]}\end{array}$ & $\mathbf{C y}$ \\
\hline $\mathbf{r s 4 6 7 3}$ & $\begin{array}{l}\text { For: CAACCCTTTGGTGCTTGTGG } \\
\text { Rev: CAAGCCCTCCTGAGCCCTA }\end{array}$ & Sequencing & 288 & 60 & 33 \\
\hline rs13058338 & $\begin{array}{l}\text { For: TTGCCCTGAGAACCAAGACC } \\
\text { Rev: CGCTGCTATTTCATGGCTGG }\end{array}$ & Sequencing & 299 & 60 & 33 \\
\hline NANOG & $\begin{array}{l}\text { For: AGTCCCAAAGGCAAACAACCCACTTC } \\
\text { Rev: ATCTGCTGGAGGCTGAGGTATTTCTGTCT } \\
\text { C }\end{array}$ & PCR & 164 & 64 & 36 \\
\hline
\end{tabular}




\begin{tabular}{|c|c|c|c|c|c|}
\hline Name & Sequence (5' $\rightarrow$ 3') & Purpose & bp & $\begin{array}{l}\mathbf{T}_{\mathrm{A}} \\
{\left[{ }^{\circ} \mathbf{C}\right]}\end{array}$ & Сy \\
\hline OCT4 & $\begin{array}{l}\text { For: GAC AAC AAT GAA AAT CTT CAG GAG A } \\
\text { Rev: TTC TGG CGC TTA CAG AAC CA }\end{array}$ & PCR & 473 & 59 & 36 \\
\hline LIN28 & $\begin{array}{l}\text { For: AGTAAGCTGCACATGGAAGG } \\
\text { Rev: ATTGTGGCTCAATTCTGTGC }\end{array}$ & PCR & 410 & 52 & 30 \\
\hline SOX2 & $\begin{array}{l}\text { For: } \text { ATG CAC CGC TAC GAC GTG A } \\
\text { Rev: CTT TTG CAC CCC TCC CAT TT }\end{array}$ & PCR & 437 & 60 & 34 \\
\hline GDF3 & $\begin{array}{l}\text { For: } \text { TTCGCTTTCTCCCAGACCAAGGTTTC } \\
\text { Rev: TACATCCAGCAGGTTGAAGTGAACAGCA } \\
\text { CC }\end{array}$ & PCR & 311 & 54 & 30 \\
\hline GAPDH & $\begin{array}{l}\text { For: } \\
\text { Rev: } \\
\text { TCTGCTGATGCAGGGATGATGTTCT } \\
\end{array}$ & $\begin{array}{l}\text { PCR, } \\
\text { qRT-PCR }\end{array}$ & 258 & 60 & $\begin{array}{l}30 / \\
40\end{array}$ \\
\hline AFP & $\begin{array}{l}\text { For: ACTCCAGTAAACCCTGGTGTTG } \\
\text { Rev: GAAATCTGCAATGACAGCCTCA }\end{array}$ & PCR & 255 & 60 & 33 \\
\hline 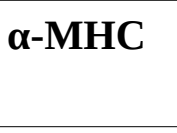 & $\begin{array}{l}\text { For: GTCATTGCTGAAACCGAGAATG } \\
\text { Rev: GCAAAGTACTGGATGACACGCT }\end{array}$ & qRT-PCR & 413 & 60 & 40 \\
\hline TH & $\begin{array}{l}\text { For: GCGGTTCATTGGGCGCAGG } \\
\text { Rev: CAAACACCTTCACAGCTCG }\end{array}$ & PCR & 215 & 60 & 34 \\
\hline cTNT & $\begin{array}{l}\text { For: GAC AGA GCG GAA AAG TGG GA } \\
\text { Rev: TGA AGG AGG CCA GGC TCT AT }\end{array}$ & $\begin{array}{l}\text { PCR, } \\
\text { qRT-PCR }\end{array}$ & 305 & 62 & $\begin{array}{l}26 / \\
40\end{array}$ \\
\hline NOX2 & $\begin{array}{l}\text { For: GCAGCCTGCCTGAATTTCA } \\
\text { Rev: TGAGCAGCACGCACTGGA }\end{array}$ & qRT-PCR & 93 & 60 & 40 \\
\hline NOX4 & $\begin{array}{l}\text { For: GCAGGAGAACCAGGAGATTG } \\
\text { Rev: CACTGAGAAGTTGAGGGCATT }\end{array}$ & qRT-PCR & 125 & 60 & 40 \\
\hline CYBA & $\begin{array}{l}\text { For: GTACTTTGGTGCCTACTCCA } \\
\text { Rev: CGGCCCGAACATAGTAATTC }\end{array}$ & qRT-PCR & 167 & 60 & 40 \\
\hline RAC1 & $\begin{array}{l}\text { For: AAGCTGACTCCCATCACCTATCCG } \\
\text { Rev: CGAGGGGCTGAGACATTTACAACA }\end{array}$ & qRT-PCR & 199 & 60 & 40 \\
\hline RAC2 & $\begin{array}{l}\text { For: CTGTCACCACCGACACTCTC } \\
\text { Rev: TGTCAAACACGGTGGGGATG }\end{array}$ & qRT-PCR & 150 & 60 & 40 \\
\hline NCF1 & $\begin{array}{l}\text { For: GTCCTGACGAGACGGAAGAC } \\
\text { Rev: TGACGTCGTCTTTCCTGATGA }\end{array}$ & qRT-PCR & 171 & 60 & 40 \\
\hline NCF2 & $\begin{array}{l}\text { For: CAGAAAGTGAACACCTTGGGG } \\
\text { Rev: GCCAAATCATATTTCTCTGTCTGGT }\end{array}$ & qRT-PCR & 382 & 60 & 40 \\
\hline NCF4 & $\begin{array}{l}\text { For: CCTCCTCAGTCGGATCAACAA } \\
\text { Rev: CTCCCAGGCCACAGACTTGAT }\end{array}$ & qRT-PCR & 181 & 60 & 40 \\
\hline
\end{tabular}




\begin{tabular}{|c|c|c|c|c|c|}
\hline Name & Sequence (5’ $\rightarrow$ 3') & Purpose & bp & $\begin{array}{l}\mathbf{T}_{\mathbf{A}} \\
{\left[{ }^{\circ} \mathbf{C}\right]}\end{array}$ & Су \\
\hline HPRT & $\begin{array}{l}\text { For: CAAAGATGGTCAAGGTCGC } \\
\text { Rev: CAAATCCAACAAAGTCTGGCT }\end{array}$ & qRT-PCR & 81 & 60 & 40 \\
\hline RYR2 & $\begin{array}{l}\text { For: TGCATGAAAGCATCAAACGCA } \\
\text { Rev: CTTGTGCAAAAACCGCAGGA }\end{array}$ & qRT-PCR & 213 & 60 & 40 \\
\hline CamKII & $\begin{array}{l}\text { For: ACCAGATGGAGTAAAGGAGTCAAC } \\
\text { Rev: AAAGCAGTAAGGCCTGGGTC }\end{array}$ & qRT-PCR & 171 & 60 & 40 \\
\hline SERCA & $\begin{array}{l}\text { For: ACAGAGTGGAAGGTGATACTTGTTC } \\
\text { Rev: AGTAAACCGACATTGACTTTCTGTC }\end{array}$ & qRT-PCR & 384 & 60 & 40 \\
\hline PLN & $\begin{array}{l}\text { For: ACAGCTGCCAAGGCTACCTA } \\
\text { Rev: GCTTTTGACGTGCTTGTTGA }\end{array}$ & qRT-PCR & 191 & 60 & 40 \\
\hline NCX & $\begin{array}{l}\text { For: AAGTGACTGAAAATGACCCTGTTAG } \\
\text { Rev: AAAAATAGTTACAGTGGCAGTGGAG }\end{array}$ & qRT-PCR & 401 & 60 & 40 \\
\hline$\alpha$-actinin & $\begin{array}{l}\text { For: } \\
\text { AGG AGG AAG AAT GGC CTG AT } \\
\text { Rev: }\end{array}$ & qRT-PCR & 291 & 60 & 40 \\
\hline$\beta$-МHC & $\begin{array}{l}\text { For: AGACTGTCGTGGGCTTGTATCAG } \\
\text { Rev: GCCTTTGCCСTTCTCAATAGG }\end{array}$ & qRT-PCR & 101 & 60 & 40 \\
\hline
\end{tabular}

bp: Base pairs; $\mathrm{T}_{\mathrm{A}}$ : Annealing temperature; Cy: Amplification cycles of PCR.

\subsection{Antibodies}

\subsubsection{Primary antibodies}

\begin{tabular}{l|l|l|l|l}
\multirow{2}{*}{ Antigen } & \multirow{2}{*}{$\begin{array}{l}\text { Host and } \\
\text { isotype }\end{array}$} & \multicolumn{2}{l}{ Dilution } & \multirow{2}{*}{ Supplier } \\
\cline { 3 - 4 } & WB & IF & \\
\hline AFP & Rabbit, IgG & - & $1: 100$ & Dako A0008 \\
\hline CamKII & Rabbit, IgG & $1: 5000$ & - & Thermo fisher scientific PA5-22168 \\
\hline cTNT & Mouse, IgG & - & $1: 500$ & $\begin{array}{l}\text { Thermo Fisher Scientific } \\
\text { GAP295PABX 13-11 }\end{array}$ \\
\hline LIN28 & Goat, IgG & - & $1: 300$ & R\&D systems AF3757 \\
\hline NANOG & Goat, IgG & - & $1: 200$ & Abcam PA5-18406 \\
\hline NCX & Rabbit, IgG & $1: 5000$ & - & Swant П 11-13 \\
\hline NOX2 & Mouse, IgG & $1: 500$ & - & $\begin{array}{l}\text { kindly provided by Dr. D. Roos, } \\
\text { Amsterdam (Burritt et al., 2000) }\end{array}$ \\
\hline
\end{tabular}


2 Materials

\begin{tabular}{|c|c|c|c|c|}
\hline NOX4 & Rabbit, IgG & $1: 500$ & - & Abcam ab109225 \\
\hline OCT4 & Goat, IgG & - & $1: 40$ & R\&D systems AF1759 \\
\hline p40phox & Rabbit, IgG & 1:1000 & - & Merck Millipore 07-503 \\
\hline PLN & Mouse, IgG & 1:5000 & - & Thermo fisher scientific MA3-922 \\
\hline PLN S16p & Rabbit, IgG & 1: 5000 & - & Badrilla A010-12AP \\
\hline $\begin{array}{l}\text { PLN } \\
\text { Thr17p }\end{array}$ & Rabbit, IgG & $1: 5000$ & - & Badrilla A010-13 \\
\hline RAC1 & Mouse, IgG & 1:1000 & - & Merck Millipore 05-389 \\
\hline RYR2 & Rabbit, IgG & 1:5000 & - & Sigma-Aldrich HPA020028 \\
\hline $\begin{array}{l}\text { RYR2 } \\
\text { S2808p }\end{array}$ & Rabbit, IgG & 1:1000 & - & Badrilla A010-30 \\
\hline $\begin{array}{l}\text { RYR2 } \\
\text { S2814p }\end{array}$ & Rabbit, IgG & $1: 5000$ & - & Badrilla A010-31 \\
\hline SERCA & Mouse, IgG & $1: 20,000$ & - & Thermo fisher scientific MA3-919 \\
\hline SOX2 & Mouse, IgG & - & $1: 50$ & R\&D systems MAB2018 \\
\hline SSEA4 & Mouse, IgG & - & $1: 200$ & Abcam MC813 \\
\hline TRA-1-60 & Mouse, IgM & - & $1: 200$ & R\&D systems MAB4770 \\
\hline$\alpha$-actinin & Mouse, IgG & $1: 10,000$ & 1:1000 & Sigma-Aldrich A7811 \\
\hline$\alpha-S M A$ & Mouse, IgG & - & $1: 3000$ & Sigma-Aldrich A2547 \\
\hline$\beta$-actin & Mouse, IgG & $1: 5000$ & - & Santa Cruz sc-47778 \\
\hline$\beta$-III-Tub & Mouse, IgG & - & 1:2000 & Covance MMS-435P \\
\hline
\end{tabular}




\subsubsection{Secondary antibodies and fluorophore-conjugated probes}

\begin{tabular}{|c|c|c|c|c|}
\hline $\begin{array}{l}\text { Antigen / } \\
\text { Probe }\end{array}$ & Conjugate & Host & Dilution & Supplier \\
\hline Mouse IgM & FITC & Goat & $1: 200$ & $\begin{array}{l}\text { Jackson ImmunoResearch } \\
\text { Laboratories 115-095-020 }\end{array}$ \\
\hline Rabbit IgG & Су3 & Goat & $1: 600$ & $\begin{array}{l}\text { Jackson ImmunoResearch } \\
\text { Laboratories 111-165-045 }\end{array}$ \\
\hline Goat IgG & Alexa Fluor 555 & Donkey & $1: 1000$ & $\begin{array}{l}\text { Thermo Fisher Scientific } \\
\text { A21432 }\end{array}$ \\
\hline Mouse IgG & Alexa Fluor 488 & Donkey & 1:1000 & $\begin{array}{l}\text { Thermo Fisher Scientific } \\
\text { A21202 }\end{array}$ \\
\hline Mouse IgG & HRP & Donkey & 1:10.000 & $\begin{array}{l}\text { Thermo Fisher Scientific } \\
\text { A16011 }\end{array}$ \\
\hline Rabbit IgG & HRP & Donkey & $1: 10.000$ & $\begin{array}{l}\text { Thermo Fisher Scientific } \\
\text { A16023 }\end{array}$ \\
\hline Phalloidin & Alexa Fluor 555 & - & $1: 200$ & $\begin{array}{l}\text { Thermo Fisher Scientific } \\
\text { A34055 }\end{array}$ \\
\hline
\end{tabular}

\subsection{DNA plasmids}

\subsection{1 pEIGW roGFP2-Orp1}

The plasmid pEIGW roGFP2-Orp1 was a gift from Tobias Dick (Addgene plasmid \#64993). It is a lentiviral vector for the mammalian expression of cytosolic roGFP2-Orp1. Details are depicted in the map of the plasmid (Figure 5). 


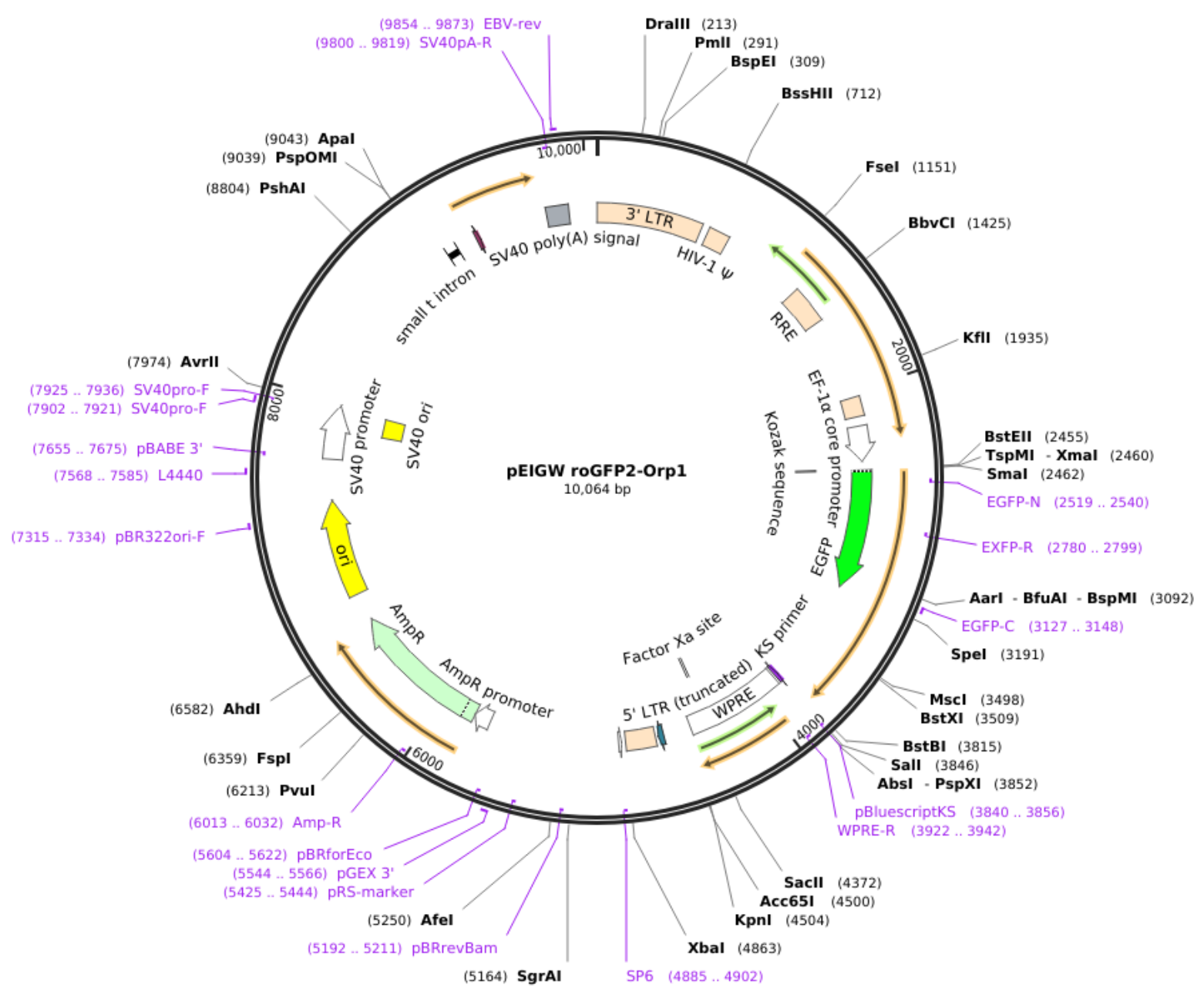

Figure 5: Map of plasmid pEIGW roGFP2-Orp1. From https://www.addgene.org/64993/ as of November 20, 2017.

\subsection{2 pGIPZ-Grx1-roGFP2}

The plasmid pGIPZ-Grx1-roGFP2 was a kind gift from Prof. Zimmermann from the institute of pharmacology and toxicology of the University Medical Center Göttingen. It was generated in the work of Dr. Heta from pLPCX-Grx1-roGFP2 (Heta, 2017). Details are depicted in the map of the plasmid (Figure 6). 


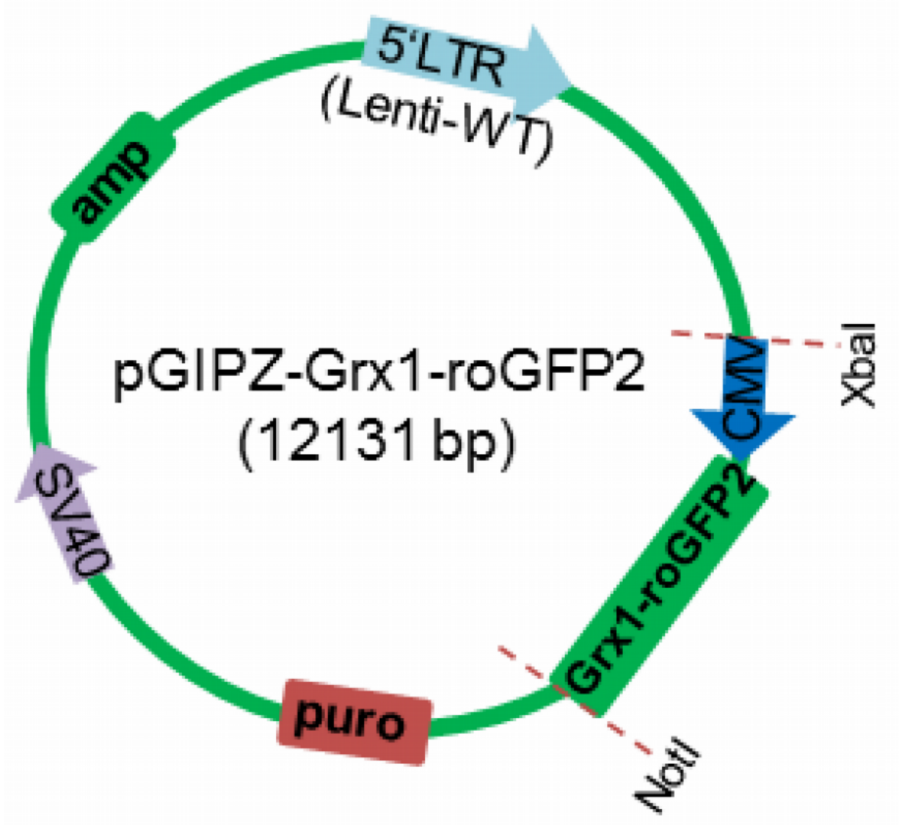

Figure 6: Map of plasmid pGIPZ-Grx1-roGFP2. From (Heta, 2017).

\subsection{3 pMD2.G}

The plasmid pMD2.G was a gift from Didier Trono (Addgene plasmid \# 12259). The vector is designed for the mammalian expression of lentiviral envelope protein VSV-G. Details are depicted in the map of the plasmid (Figure 7). 


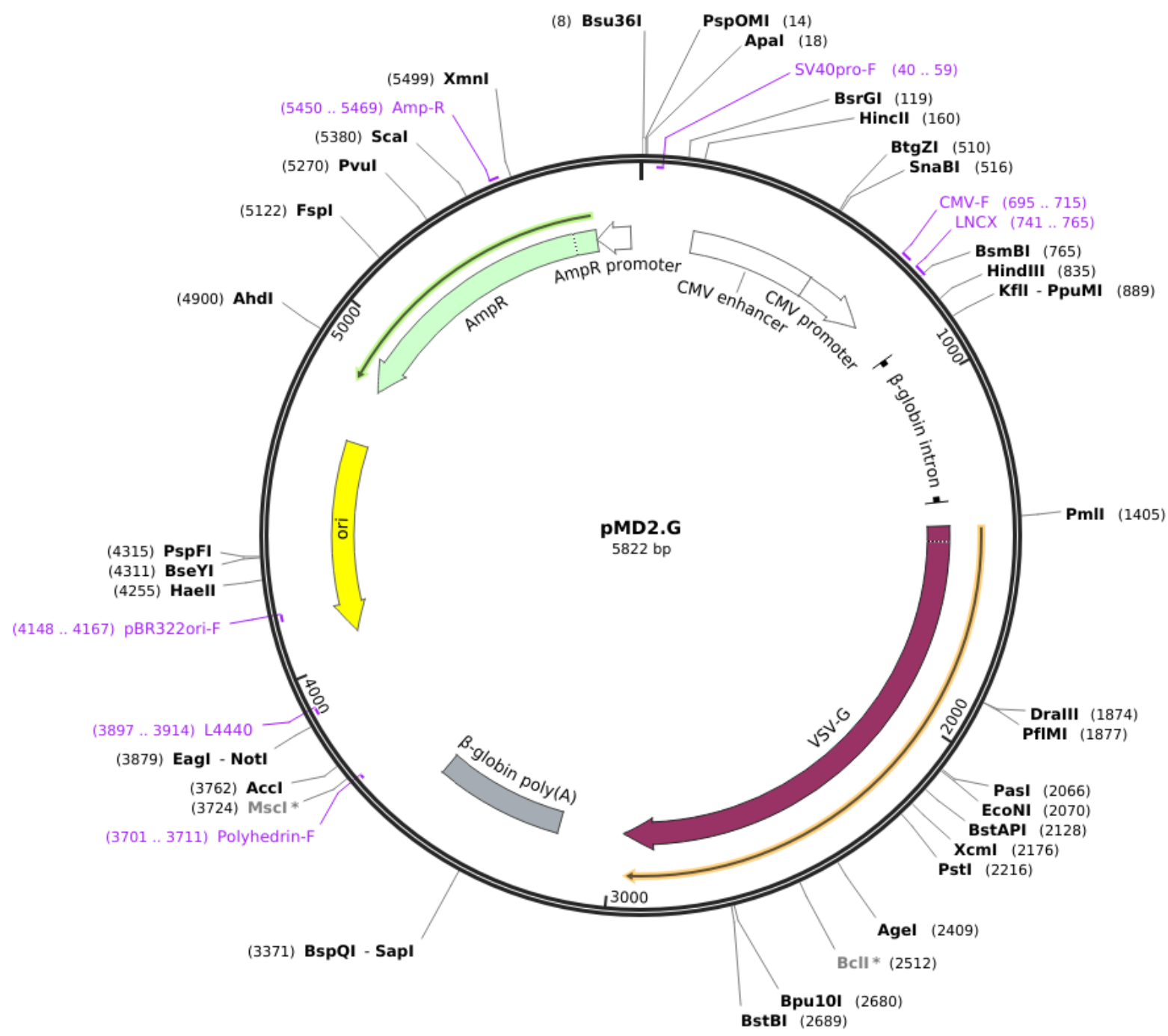

Figure 7: Map of plasmid pMD2.G. From https://www.addgene.org/12259/ as of November 20, 2017.

\subsection{4 psPAX2}

The plasmid psPAX2 was a gift from Didier Trono (Addgene plasmid \# 12260). The vector is designed for the mammalian expression of lentiviral packaging proteins. Details are depicted in the map of the plasmid (Figure 8). 


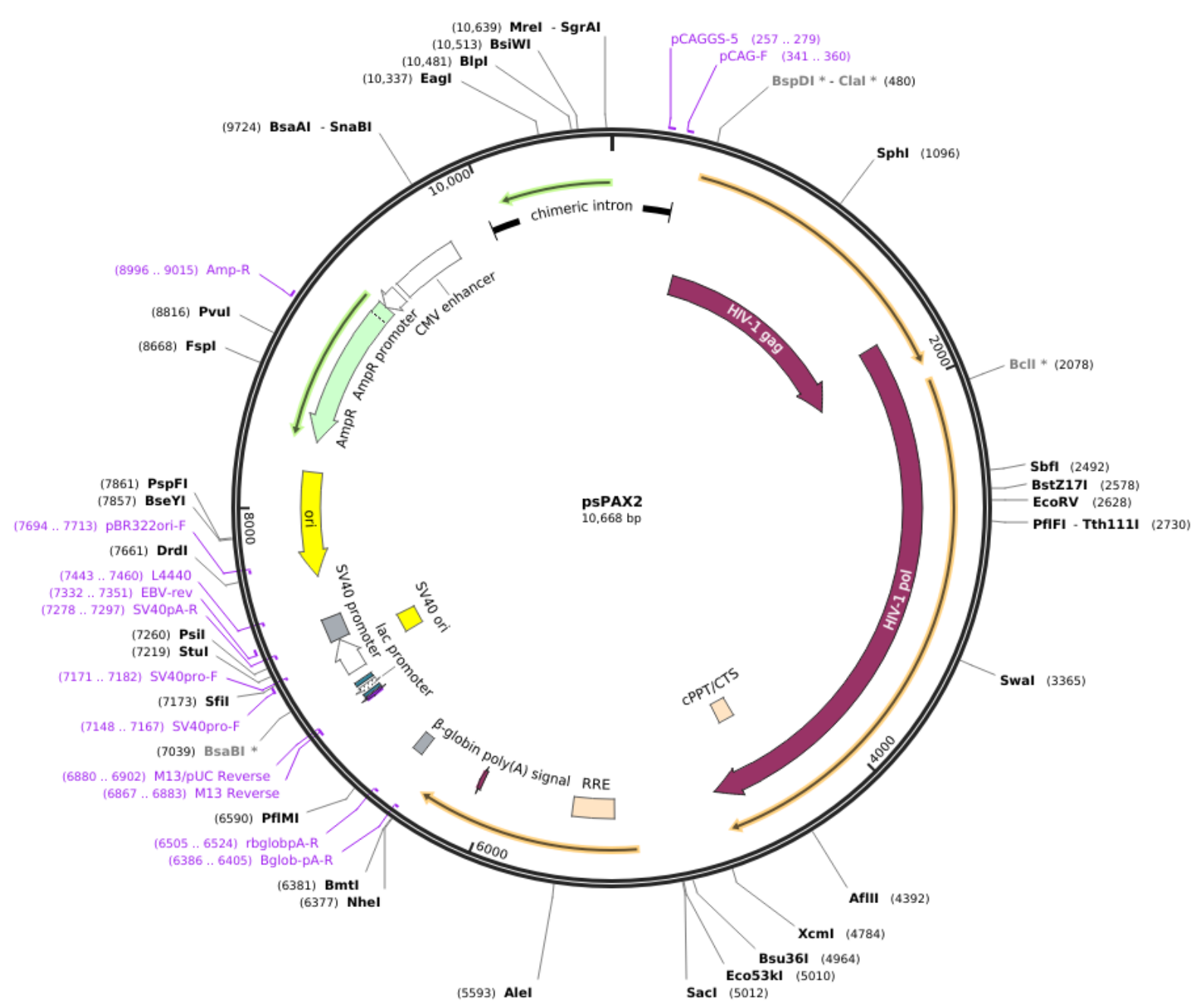

Figure 8: Map of plasmid psPAX2. From https://www.addgene.org/12260/ as of November 20, 2017.

\subsection{Disposable items}

\begin{tabular}{l|l} 
Name & Provider \\
\hline 10-cm dish, TC-treated & CytoOne Starlab \#CC7682-3394 \\
\hline 12-well plate, TC-treated & CytoOne Starlab \#CC7682-7512 \\
\hline 6-cm dish, TC-treated & CytoOne Starlab \#CC7682-3359 \\
\hline 6-cm dish, untreated & Sarstedt \#82.1194.500 \\
\hline 6-well plate, TC- treated & CytoOne Starlab \#CC7682-7506 \\
\hline 96 -well microplate, black & Berthold Technologies \#23302 \\
\hline 96 -well microplate, black, clear bottom & Berthold Technologies \#38840 \\
\hline Cell scraper: 2-Posit. Blade 25 & Sarstedt \#83.1830 \\
\hline
\end{tabular}




\begin{tabular}{|c|c|}
\hline Name & Provider \\
\hline Filter tips: $0.1-1000 \mu \mathrm{L}$ & $\begin{array}{l}\text { Starlab \#S1120-3810, \#S1122-1830, } \\
\text { \#S1120-1840 }\end{array}$ \\
\hline $\begin{array}{l}\text { Flow cytometry tube: } 5 \mathrm{~mL} \text { Polystyrene Round- } \\
\text { Bottom Tubes }\end{array}$ & BD Falcon \#352058 \\
\hline Pipette tips: $0.1-1000 \mu \mathrm{L}$ & $\begin{array}{l}\text { Starlab \#S1111-3700, \#S1111-1706, } \\
\text { \#S1112-1720 }\end{array}$ \\
\hline Pipettes: 5 mL, 10 mL, 25 mL & $\begin{array}{l}\text { Sarstedt \#86.1253.001, } \\
\# 86.1254 .001, \text { \#86.1685.001 }\end{array}$ \\
\hline $\begin{array}{l}\text { PVDF membrane: Amersham Hybond P Western } \\
\text { blotting membranes }\end{array}$ & Sigma-Aldrich \#GE10600023 \\
\hline $\begin{array}{l}\text { Slides and coverslips: } 76 \text { × } 26 \text { mm, } 18 \text { x } 18 \text { mm, } \\
\text { round } 25 \text { mm }\end{array}$ & $\begin{array}{l}\text { Thermo Fisher Scientific } \\
\text { \#10143562CE, Thermo Fisher } \\
\text { Scientific \#4004672; R. } \\
\text { Langenbrinck, \#1049251 }\end{array}$ \\
\hline $\begin{array}{l}\text { Sterile filters: Millex-GS, } 0.22 \mu \mathrm{m} \text {; Steriflip } 50 \mathrm{~mL} \text {, } \\
0.22 \mu \mathrm{m} \text {; Steritops } 500 \mathrm{~mL}, 0.22 \mu \mathrm{m}\end{array}$ & $\begin{array}{l}\text { Merck Millipore \#GLGS0250S } \\
\text { Merck Millipore \#SCGP00525 } \\
\text { Merck Millipore \# SCGPT05RE }\end{array}$ \\
\hline Whatman gel blotting paper, Grade GB003 & Sigma-Aldrich \#WHA10426890 \\
\hline
\end{tabular}

\subsection{Laboratory equipment}

\begin{tabular}{l|l} 
Name & Provider \\
\hline Balances: Extend ED153-CW, CPA225D & Sartorius \\
\hline Blotting chamber: Trans-Blot Turbo Transfer System & Bio-Rad \\
\hline Cell stimulator: MyoPacer ES & IonOptix \\
\hline Centrifuges: 5810R, 5415D, 5415R & Eppendorf \\
\hline $\begin{array}{l}\text { Chemiluminescence detection system: ChemiDoc } \\
\text { MP Imaging System Universal Hood III }\end{array}$ & Bio-Rad \\
\hline $\begin{array}{l}\text { Confocal laserscanning microscope: LSM 710 } \\
\text { confocal microscopic system }\end{array}$ & Carl Zeiss \\
\hline Counting chamber: Neubauer improved & Marienfeld Superior \\
\hline DNA Isolation: DNA Maxwell 16 System & Promega \\
\hline $\begin{array}{l}\text { Electrophoresis chambers: Mini-PROTEAN Tetra } \\
\text { Vertical Electrophoresis Cell }\end{array}$ & Bio-Rad \\
\hline Flow cytometer: FACS Canto II & \\
\hline Freezing box: Mr. Frosty & BD Biosciences \\
\hline Gel documentation: Multilmage Light Cabinet & Thermo Fisher Scientific \\
\hline
\end{tabular}




\begin{tabular}{l|l} 
Name & Provider \\
\hline \hline Incubator: HERACELL VIOS 160i CO2 Incubator & Thermo Fisher Scientific \\
\hline Microplate reader: Mithras LB 940, Mithras² LB 943 & Berthold Technologies \\
\hline $\begin{array}{l}\text { Microscopes: Axio Oberserver A1, Axio Oberserver } \\
\text { Z1, Primo Vert, Axiovert 25 }\end{array}$ & Carl Zeiss \\
\hline NanoDrop 2000c/2000 UV-Vis Spectrophotometer & Thermo Fisher Scientific \\
\hline PCR cycler: Thermocycler 48 & SensoQuest \\
\hline pH meter: inoLab pH 7110 & WTW \\
\hline Pipet controller: Accu-jet pro & Brand \\
\hline Pipettes: Reference, Research plus (10/100/1000 $\mu \mathrm{L})$ & Eppendorf \\
\hline Power supply: Power Pac 3000-Power supply & Bio-Rad \\
\hline qPCR cycler: iCycler Thermal Cycler, iQ5 & Bio-Rad \\
Multicolor Real-Time PCR Detection System & \\
\hline Sterile work bench: & CleanAir CA/RE 5 \\
\hline $\begin{array}{l}\text { Tissue embedding system: Benchtop Tissue } \\
\text { Processor 1020 }\end{array}$ & Leica Biosystems \\
\hline $\begin{array}{l}\text { Water preparation system } \\
\text { Milli-Q Reference }\end{array}$ & Merck Millipore \\
\hline
\end{tabular}

\subsection{Software}

\begin{tabular}{l|l} 
Name & Provider / Author \\
\hline ImageJ / FIJI & $\begin{array}{l}\text { National Institutes of Health (Schindelin } \\
\text { et al., 2012, 2015; Schneider et al., 2012) }\end{array}$ \\
\hline Prism 6/7 & GraphPad Software, Inc \\
\hline LabChart & AD Instruments \\
\hline Mikrowin 2000/2010 & Mikrotek Laborsysteme GmbH \\
\hline ApE A plasmid editor & M. Wayne Davis \\
\hline LibreOffice & The Document Foundation \\
\hline Inkscape & Inkscape Community \\
\hline AxioVision & Carl Zeiss \\
\hline Zen & Carl Zeiss \\
\hline IQ optical system software & Bio-Rad \\
\hline Flowing Software & Perttu Terho \\
\hline Image Lab Software & Bio-Rad \\
\hline Office & Microsoft
\end{tabular}




\section{Methods}

\subsection{Cell culture}

All cells were cultured under humidified air conditions with $5 \% \mathrm{CO}_{2}$ and $20 \% \mathrm{O}_{2}$ at $37^{\circ} \mathrm{C}$.

\subsubsection{Isolation and cultivation of human primary dermal fibroblasts}

Donor skin biopsies were sent to the University Medical Center Göttingen in $15 \mathrm{~mL}$ conical tubes containing DMEM and $1 \mathrm{x} / \mathrm{S}$. Using scalpels, the dermis was separated from the epidermis and subcutaneous layer and was cut into pieces of about $1 \mathrm{~mm}^{2}$. hFB-medium was added and the biopsies were cultured on $6-\mathrm{cm}$ dishes for about one week without medium change to ensure attachment and proliferation of fibroblasts. Primary fibroblasts were cultured in hFB-medium, which was replaced every second day and the cells were passaged every four to six days. For this purpose, the cells where quickly washed with $0.1 \%$ Trypsin/EDTA and afterwards shortly incubated in 0.1\% Trypsin/EDTA at room temperature until cells started to detach. Finally, the Trypsin/EDTA was carefully removed and the cells were detached from the dish using hFB-medium and transferred into new dishes in an appropriate ratio.

\subsubsection{Isolation and cultivation of mouse embryonic fibroblasts}

Mouse embryos at stage E14.5 were used to isolate MEFs (Wobus et al., 2002), which were cultured on $0.1 \%$ gelatine-coated dishes up to passage number 4 in MEF-medium. The cells were mitotically inactivated by treatment with $10 \mu \mathrm{g} / \mathrm{mL} M M C$ for $3 \mathrm{~h}$. Then they were plated on $0.1 \%$ gelatine-coated dishes in an appropriate ratio. The MEFs were cultured overnight and used as feeder layer for hiPSCs.

\subsubsection{Generation of hiPSC lines}

hiPSCs were generated from primary dermal fibroblasts using the CytoTune-iPS 2.0 Sendai Reprogramming Kit (Thermo Fisher Scientific). For this purpose, $7.5 \times 10^{4}$ cells were plated per well of a 6 -well culture plate and two wells were prepared. Two days later, cells from one well were trypsinyzed as described above and counted to estimate the cell number of the remaining well. The required amount of virus was calculated with $\mathrm{MOI}$ (multiplicity of infection in $\mathrm{CIU}$ (collective infectious unit) per cell)) of 5 for the viruses KOS and hc-Myc and 3 for hKIf4:

$$
\text { Volume of virus }[\mu L]=\frac{\text { MOI }[\text { CIU / cell }] \cdot \text { number of cells }}{\text { titer of virus }[\mathrm{CIU} / \mathrm{mL}] \cdot 10^{-3}[\mu L / \mathrm{mL}]} \text {. }
$$


The titer of each virus was lot-dependent. To thaw the viruses, the bottom of the tube was immersed in a $37^{\circ} \mathrm{C}$ water bath for $10 \mathrm{sec}$. Subsequently, the virus was thawed at room temperature and shortly centrifuged. The calculated amount of each virus was added to $1 \mathrm{~mL}$ pre-warmed hFB-medium and carefully mixed by pipetting. Afterwards, the cell medium was aspirated from the remaining attached cells and the prepared virus suspension was added. $24 \mathrm{~h}$ after transduction, medium was replaced with hFB-medium every day. On day 7 after transduction, cells were trypsinyzed as described above and transferred onto geltrex-coated 6 -cm culture dishes in an appropriate ratio. The medium was changed to E8-medium at day 8 after transduction and replaced daily. Emerging iPSC-like colonies of appropriate size were manually transferred onto geltrex-coated 12-well culture plates about two to three weeks after transduction.

\subsubsection{Cultivation of hiPSCs on MEFs or geltrex}

hiPSCs were either cultured on MMC-inactivated MEFs in hES-medium or on geltrex in E8medium and the medium was replaced every day. During the cocultivation with MEFs, passaging was done every five to seven days, depending on the density and size of hiPSC colonies. Therefore, hiPSCs were washed once with DMEM/F12 and subsequently incubated in collagenase IV $(200 \mathrm{U} / \mathrm{mL})$ at $37^{\circ} \mathrm{C}$ for $5 \mathrm{~min}$. After washing the cells two times with DMEM/F12, they were mechanically detached from the culture dish in hES-medium using a cell scraper. To receive appropriately sized cell aggregates, the cells were resuspended one to three times with a $1000 \mu \mathrm{L}$ handpipette and transferred in an appropriate ratio onto $6-\mathrm{cm}$ culture dishes containing MMC-inactivated MEFs and hES-medium.

To adapt hiPSCs to feeder-free culture, they were processed as described before and transferred onto geltrex-coated 6-well culture plates. To passage hiPSCs that were cultured on geltrex, the cells were washed two times with versene. Afterwards, they were incubated in versene for $3-5 \mathrm{~min}$ at room temperature. The versene was carefully aspirated and the cells were detached from the dish using E8-medium with $2 \mu \mathrm{M}$ TZV and resuspended three to five times. Finally, an appropriate amount of cells were transferred onto geltrex-coated 6well plates containing E8-medium with $2 \mu \mathrm{M}$ TZV.

\subsubsection{Freezing of dermal fibroblasts and iPSCs on MEFs and geltrex}

hiPSCs and fibroblasts were frozen in liquid nitrogen for long-term storage. In the case of hiPSCs cocultured with MEFs, colonies were frozen five to seven days after passaging when they reached $70-80 \%$ confluence. Fibroblasts and feeder-free hiPSCs were frozen about four days after passaging when they reached $80-100 \%$ confluence. HiPSCs and fibroblasts were detached from the culture dish as described in chapter 3.1.1 and 3.1.4 using Trypsin/EDTA, collagenase IV or Versene, respectively. Afterwards, fibroblasts and iPSCs that were cultured 
on MEFs were transferred into $15 \mathrm{~mL}$ conical tubes with basal medium and centrifuged for 3 $\min$ at $200 \times \mathrm{g}$. The cell pellet was carefully resuspended in $1 \mathrm{~mL}$ freezing medium 1 and transferred into a $2 \mathrm{~mL}$ cryovial. Finally, the cryovial was frozen for at least $24 \mathrm{~h}$ at $-80^{\circ} \mathrm{C}$ using a Nalgene Cryo $1^{\circ} \mathrm{C}$ Freezing Container and was placed into liquid nitrogen storage afterwards.

hiPSCs that were cultured on geltrex were resuspended in $1 \mathrm{~mL}$ E8-medium per 6-well after detachment. Afterwards, $1 \mathrm{~mL}$ freezing medium 2 was added drop-wise and the cell solution was transferred equally into two $2 \mathrm{~mL}$ cryovials. The cryovials were stored in the same way as described above.

\subsubsection{Thawing of HEK cells and iPSCs on MEFs or geltrex}

To thaw cells, the cryovial was placed in a $37^{\circ} \mathrm{C}$ water bath immediately after removal from liquid nitrogen storage for about $1 \mathrm{~min}$. The cell suspension was transferred gently into a $15 \mathrm{~mL}$ conical tube containing $10 \mathrm{~mL}$ of cold basal medium (DMEM or DMEM/F12) and centrifuged for $3 \mathrm{~min}$ at $200 \times \mathrm{g}$. The supernatant was removed and the cell pellet was carefully resuspended in the respective culture medium. Finally, the cells were transferred onto respective culture dishes (see chapter 3.1.1 and 3.1.4). $2 \mu \mathrm{M}$ TZV was added to the culture medium of iPSCs cultured on geltrex.

\subsubsection{Spontaneous in vitro differentiation}

Differentiating hiPSC in vitro was done to observe their potential to give rise to derivatives of all three germ layers and to analyze the efficiency of cardiac lineage differentiation. A spontaneous differentiation protocol was applied without the use of factors that direct the differentiation into a specific cell type. First, at day 0 hiPSCs of about $80 \%$ confluence on MEFs were detached from the culture dish using collagenase IV as described above (see chapter 3.1.4). The cell clumps were transferred with hES-medium into a non-coated 6-cm suspension culture dish and incubated at $37^{\circ} \mathrm{C}$ over-night. When embryoid bodies (EBs) had formed, they were transferred with the culture medium into a $15 \mathrm{~mL}$ conical tube. Subsequently to the sedimentation of the EBs, they were carefully washed with IMDM and transferred into a non-coated 6 -cm suspension culture dish with Iscove medium. The EBs were cultured in suspension at $37^{\circ} \mathrm{C}$ for 8 days and the medium was changed on day 4 and 7 after differentiation start. On day 8 , the EBs that were to be analyzed by semiquantitative RTPCR were plated on $0.1 \%$ gelatin-coated culture plates. For immunofluorescence analyses EBs were plated on $0.1 \%$ gelatin-coated culture plates containing glass cover-slips. About 30 EBs were transferred into a 6-cm culture dish. The EBs were cultured for another 25 days in Iscove medium, which was replaced every two days. To analyze the efficiency of cardiac lineage differentiation, the number of plated EBs and EBs which developed beating areas 
were counted. The counting was usually performed at days 11, 14, 18, 25 and 33 after differentiation start.

\subsubsection{Cardiac differentiation of hiPSCs and metabolic selection}

To obtain iPSC-CMs the iPSCs were plated on geltrex-coated 12-well plates and cultured for two to four days in E8 medium until they reached about $90 \%$ confluency. To start the differentiation, the E8 medium was replaced by differentiation medium containing $4 \mu \mathrm{M}$ CHIR. $48 \mathrm{~h}$ later, the medium was replaced by differentiation medium with $5 \mu \mathrm{M}$ IWP2. On day 4 and 6 after differentiation start, the medium was replaced by differentiation medium. The medium was changed to cardiac culture medium on day 7 to 9 when first beating or CMlike cells were visible. From there on, the medium was replaced every 2 to 3 days.

Three to four weeks after differentiation start, the cells were singularized by $15 \mathrm{~min}$ incubation with $0.25 \%$ Trypsin/EDTA at $37^{\circ} \mathrm{C}$ and resuspension. Afterwards, the cell suspension was diluted in two volumes of digestion medium and centrifuged at $200 \mathrm{x} g$ for 3 min. After removing the supernatant, the cells were plated onto geltrex-coated 6-well pates in a ratio appropriate to obtain a monolayer of CMs. Two days after recovery in digestion medium, the CMs were purified by cultivation in selection medium for two to eight days until only beating CMs were remaining. From there on, iPSC-CMs were cultured in cardiac culture medium which was replaced every two to three days.

Two to three month after differentiation start, the iPSC-CMs were used for analyses. For this purpose, they were singularized by treatment with $0.25 \%$ Trypsin/EDTA for $5 \mathrm{~min}$ as described above, pooled and plated in ratios and onto geltrex-coated culture plates as needed for the respective methodology. After one week of cultivation the iPSC-CMs recovered from digestion and were processed.

\subsubsection{Preparation and preservation of cell samples for analyses}

For long-term storage of cell samples, cells were washed three times with DPBS, detached from the culture dish with a cell scraper and transferred into a $1.5 \mathrm{~mL}$ conical tube. After centrifugation at $200 \times \mathrm{g}$ for $3 \mathrm{~min}$, the supernatant was removed and the tube was incubated in liquid nitrogen for several minutes to snap-freeze the cells. The samples were stored at $-80^{\circ} \mathrm{C}$.

\subsubsection{Cultivation of HEK-293T cells}

HEK-293T cells were cultured on 10-cm culture dishes in HEK-medium, which was replaced every three days. For passaging, the cells were washed once with DPBS and incubated in TrypLE Express for about 3 min until the cells started to detach. After adding HEK-medium, 
the cells were singularized by pipetting and transferred onto fresh culture dishes in an appropriate ratio.

\subsubsection{Lentivirus production}

To obtain roGFP2-Orp1 or Grx1-roGFP2 containing lentiviruses, HEK-293T cells were transfected with plasmid DNA using Polyfect (Qiagen). For this purpose, 70\% confluent cells, which were plated about two days prior, were washed once with PBS and $7 \mathrm{~mL}$ HEK transfection-medium was added. $3 \mu \mathrm{g}$ target vector, $3 \mu \mathrm{g}$ vector psPAX2, $2 \mu \mathrm{g}$ vector pMD2.G and $80 \mu \mathrm{L}$ Polyfect were added to Opti-MEM medium to receive a final volume of $300 \mu \mathrm{L}$. After addition of $80 \mu \mathrm{L}$ Polyfect, the suspension was immediately vortexed for $10 \mathrm{sec}$ and incubated for $10 \mathrm{~min}$ at room temperature. Subsequently, $620 \mu \mathrm{L}$ HEK transfection-medium was added and the transfection mixture was transferred drop-wise to the HEK-293T cells. After three days of incubation, debris was removed from the cell supernatant by filtering with $0.45 \mu \mathrm{m}$ syringe filters and the flow-through was stored at $4^{\circ} \mathrm{C} .10 \mathrm{~mL}$ HEK transfectionmedium was added to the transfected cells which were incubated for another $24 \mathrm{~h}$. The cell supernatant was filtered as before and added to the previous flow-through. Finally, $2 \mathrm{~mL}$ aliquots of virus containing cell supernatant were prepared, snap-frozen in liquid nitrogen for several minutes and stored at $-80^{\circ} \mathrm{C}$.

\subsubsection{Lentiviral transduction of iPSC-CMs}

The lentivirus containing HEK-293T cell supernatant was thawed at room temperature. For transduction, a virus containing suspension was made, consisting of one volume HEK-293T cell supernatant, five volumes cardio culture medium and $6 \mu \mathrm{g} / \mathrm{mL}$ polybrene. For reduction-oxidation-sensitive GFP2 (roGFP2)-based biosenor measurements, $1.5 \times 10^{4}$ iPSCCMs per well were cultured for one week and transduced in 96-well culture plates with black walls and clear bottoms. $300 \mu \mathrm{L}$ virus suspension was added per well and incubated for three days. Afterwards, medium was replaced by cardio culture medium and cells were used for analyses two days later if a sufficient amount expressed the biosensors as observed by fluorescence microscopy.

\subsubsection{Engineered heart muscle}

The generation of EHMs and experiments were done in cooperation with the institute of pharmacology and toxicology of the University Medical Center Göttingen by the group of Dr. med. Malte Tiburcy. To generate an EHM, iPSC-CMs and human foreskin fibroblasts (ATCC) were reconstituted in a ratio of $70 / 30 \%$ in EHM-reconstitution mixture. The cells were cultured for 3 days in EHM-culture medium. Subsequently, EHMs were transferred to flexible holders. Analyses were performed on 4-week old EHMs by Irina Eckhardt. 


\subsection{Molecular biology techniques}

\subsubsection{Isolation of genomic DNA and sequencing of SNPs}

The Maxwell 16 instrument and the Maxwell 16 DNA Purification Kit (Promega) were used to automatically isolate genomic DNA from fibroblasts. A stored cell sample (see chapter 3.1.9) was resuspended in $350 \mu \mathrm{L}$ DPBS and transferred into the respective well of a cartridge. $300 \mu \mathrm{L}$ elution buffer was placed into the elution tube. After loading the plunger into the respective well, the cartridge was placed into the Maxwell 16 instrument and the program "cells" was run. Remaining beats were filtered with a magnetic rack and the eluate was transferred into a conical $1.5 \mathrm{~mL}$ tube. The DNA concentration was assessed photospectroscopically at $260 / 280 \mathrm{~nm}$.

100 ng DNA was used as template in a polymerase chain reaction (see chapter 3.2.3.3) to amplify a DNA fragment, containing the SNP of interest. The PCR product was purified for sequencing using the QIAquick Gel Extraction Kit (Qiagen). Therefore, three volumes of buffer QG was mixed with one volume of PCR product and one volume of isopropanol and transferred into a QIAquick column. After centrifugation at $13.000 \times \mathrm{g}$ for $1 \mathrm{~min}$, the flowthrough was discarded and the DNA washed by addition of $750 \mu \mathrm{L}$ buffer PE to the column and centrifugation at $13.000 \times \mathrm{g}$ for $1 \mathrm{~min}$. The flow-through was discarded and the column was centrifuged dry as before. The DNA was eluted into a fresh conical $1.5 \mathrm{~mL}$ tube by addition of $50 \mu \mathrm{L}$ nuclease-free water and centrifugation as before. Subsequently the DNA concentration was measured photospectroscopically at 260/280 nm. A mix containing 67.5 ng DNA and $10 \mathrm{pmol}$ forward primer in a final volume of $15 \mu \mathrm{L}$ nuclease-free water was made and sequencing was performed by Seqlab Sequencing Laboratories $\mathrm{GmbH}$, Göttingen. The generated chromatograms were analyzed with the software "ApE, A plasmid Editor" by M. Wayne Davis (http://biologylabs.utah.edu/jorgensen/wayned/ape/; as of October 10, 2017).

\subsubsection{Alkaline phosphatase staining}

To observe the activity of the alkaline phosphatase in hiPSCs and iPSC-like colonies after transduction, the Alkaline Phosphatase Kit (Sigma-Aldrich) was used. The fixative was prepared in a glass flask by mixing $5 \mathrm{~mL}$ citrate solution with $13 \mathrm{~mL}$ acetone and $1.6 \mathrm{~mL} \mathrm{37 \%}$ formaldehyde. To prepare the staining solution, one volume of the provided sodium nitrate was mixed with one volume of FRV-Alkaline solution and incubated at room temperature for $2 \mathrm{~min} .45$ volumes of water were added and one volume of Naphthol AS-B was added shortly before usage. The cells were washed with DPBS, incubated in fixative solution for $30 \mathrm{sec}$ at room temperature and subsequently washed two times with deionized water. Staining 
solution was added for $15 \mathrm{~min}$ at $37^{\circ} \mathrm{C}$ in the dark. Finally, the cells were washed two times with deionized water and air-dried.

\subsubsection{Gene expression analysis}

\subsubsection{Isolation of mRNA}

To observe the gene expression of specific markers in hiPSCs, differentiated EBs and human primary fibroblasts on the mRNA level, RT-PCR analysis was performed. The SV Total RNA Isolation System (Promega) was used to isolate RNA. For this purpose, undifferentiated hiPSCs of at least $70 \%$ confluency, $100 \%$ confluent fibroblasts and differentiated EBs were washed three times with DPBS and were resuspended in 400-600 $\mu \mathrm{L}$ RNA-lysis buffer supplemented with $\beta$-ME $(200 \mu \mathrm{L} / 10 \mathrm{~mL})$. The resulting lysate could be stored at $-20^{\circ} \mathrm{C}$. The lysate was mixed in a 1:1 ratio with $95 \%$ ethanol. The solution was transferred into the Spin Basket Assembly and centrifuged at 12,000 x g for $1 \mathrm{~min}$. The flow-through was discarded and $600 \mu \mathrm{L}$ RNA wash solution was added. To prepare the DNase mix, $40 \mu \mathrm{L}$ Yellow core buffer, $5 \mu \mathrm{L} 0.09 \mathrm{M} \mathrm{MnCl}_{2}$ and $5 \mu \mathrm{L}$ DNase I were mixed. After centrifugation and removal of the flow-through, $50 \mu \mathrm{L}$ of DNase mix was added and incubated at room temperature for 15 min. Subsequently, $200 \mu \mathrm{L}$ of DNase stop solution was added and the Spin Basket Assembly was centrifuged. Two washing steps were performed by first adding $600 \mu \mathrm{L}$ RNA wash solution and centrifuging for $1 \mathrm{~min}$ and thereafter adding $250 \mu \mathrm{L}$ RNA wash solution and centrifuging for $2 \mathrm{~min}$. Finally, $100 \mu \mathrm{L}$ of nuclease free water was added and the RNA was eluted by centrifuging for $1 \mathrm{~min}$. The RNA concentration was measured with a spectrophotometer at $260 / 280 \mathrm{~nm}$ and the samples were stored at $-80^{\circ} \mathrm{C}$.

\subsubsection{Reverse transcription}

In the reverse transcription reaction the reverse transcriptase uses the isolated RNA as a template to generate complementary DNA (cDNA). For this purpose, the following reaction mix was prepared: 


\begin{tabular}{ll} 
RNA & $100 \mathrm{ng}$ \\
10x PCR buffer II & $2 \mu \mathrm{L}$ \\
$25 \mathrm{mM} \mathrm{MgCl}_{2}$ & $4 \mu \mathrm{L}$ \\
$100 \mathrm{mM}$ dNTPs & $0.8 \mu \mathrm{L}$ \\
RNase Inhibitor $(20 \mathrm{U} / \mu \mathrm{L})$ & $1 \mu \mathrm{L}$ \\
$50 \mu \mathrm{M}$ Oligo $(\mathrm{dt})_{16}$ & $1 \mu \mathrm{L}$ \\
MuLV Reverse Transcriptase $(50 \mathrm{U} / \mu \mathrm{L})$ & $1 \mu \mathrm{L}$ \\
Nuclease free $\mathrm{H}_{2} \mathrm{O}$ & $\mathrm{x} \mu \mathrm{L}$ \\
\hline Final volume & $20 \mu \mathrm{L}$
\end{tabular}

The reaction was run in a thermal cycler (SensoQuest) using the following program.

\begin{tabular}{l|l|l|l|l} 
& Step 1 & Step 2 & Step 3 & Step 4 \\
\hline Temperature & $22^{\circ} \mathrm{C}$ & $42^{\circ} \mathrm{C}$ & $95^{\circ} \mathrm{C}$ & $4^{\circ} \mathrm{C}$ \\
\hline Time & $10 \mathrm{~min}$ & $50 \mathrm{~min}$ & $10 \mathrm{~min}$ & $\infty$
\end{tabular}

\subsubsection{Polymerase chain reaction (PCR) analysis}

The CDNA that was generated in the reverse transcription reaction was amplified by PCR using primers specific for the analyzed genes (see chapter 2.7). Following reaction mix was prepared:

\begin{tabular}{ll} 
cDNA & $1 \mu \mathrm{L}$ \\
5x Green GoTaq Reaction Buffer I & $5 \mu \mathrm{L}$ \\
$10 \mathrm{mM}$ dNTPs & $1.6 \mu \mathrm{L}$ \\
Primer forward $(10 \mathrm{pmol} / \mu \mathrm{L})$ & $1 \mu \mathrm{L}$ \\
Primer reverse $(10 \mathrm{pmol} / \mu \mathrm{L})$ & $1 \mu \mathrm{L}$ \\
GoTaq DNA Polymerase & $0.1 \mu \mathrm{L}$ \\
Nuclease free $\mathrm{H}_{2} \mathrm{O}$ & $\mathrm{x} \mu \mathrm{L}$ \\
\hline Final volume & $25 \mu \mathrm{L}$
\end{tabular}

The PCR reactions were run according to the following general program. For details on the annealing temperatures and cycle repeats see chapter 2.7 . 


\begin{tabular}{l|l|l|l} 
Step & Temperature $\left[{ }^{\circ} \mathrm{C}\right]$ & Time & Cycles \\
\hline Denaturation & \multirow{2}{*}{95} & $3 \mathrm{~min}$ & 0 \\
\cline { 3 - 3 } & & $15 \mathrm{sec}$ & \\
\hline Annealing & $52-65$ & $15 \mathrm{sec}$ & \multirow{2}{*}{$30-43$} \\
\hline Elongation & \multirow{2}{*}{72} & $30 \mathrm{sec}$ & \\
\cline { 3 - 3 } & & $10 \mathrm{~min}$ & 0
\end{tabular}

The PCR product could be stored at $4^{\circ} \mathrm{C}$. To observe the amplified DNA fragments, agarose gel electrophoresis was performed. For this purpose, a 1.5\% agarose gel containing $6 \mu \mathrm{L}$ Midori Green per $100 \mathrm{~mL}$ gel was loaded with $15 \mu \mathrm{L}$ PCR product and $7 \mu \mathrm{L}$ GeneRuler $100 \mathrm{bp}$ Plus DNA Ladder (Fermentas). The DNA fragments were separated at $100 \mathrm{~V}$ for about $30 \mathrm{~min}$ depending on the gel size and visualized with UV light.

\subsubsection{Quantitative real-time polymerase chain reaction (qRT-PCR) analysis}

For quantification of specific mRNA by real-time PCR a serial dilution of the respective DNA was made, to get standards of known concentration. For this purpose, a PCR (see chapter 3.2.3.3) was performed with template CDNA that contained the gene of interest and the PCR product was purified (see chapter 3.2.1) subsequently. After measuring the concentration of DNA, standards with concentrations ranging between $1 \mathrm{ng} / \mu \mathrm{L}$ to $0.125 \mathrm{fg} / \mu \mathrm{L}$ were produced and stored at $-20^{\circ} \mathrm{C}$.

As described above, mRNA was isolated from samples of interest (see chapter 3.2.3.1) and cDNA was generated (see chapter 3.2.3.2). For qRT-PCR the following reaction mix was prepared for one well of a 96-well plate:

\begin{tabular}{ll} 
cDNA & $1 \mu \mathrm{L}$ \\
SYBR Green Mix & $10 \mu \mathrm{L}$ \\
Primer forward $(10 \mathrm{pmol} / \mu \mathrm{L})$ & $1 \mu \mathrm{L}$ \\
Primer reverse $(10 \mathrm{pmol} / \mu \mathrm{L})$ & $1 \mu \mathrm{L}$ \\
Nuclease free $\mathrm{H}_{2} \mathrm{O}$ & $7 \mu \mathrm{L}$ \\
\hline Final volume & $20 \mu \mathrm{L}$
\end{tabular}

Duplicates were pipetted for each sample and standard. The DNA was amplified using following program on a real-time PCR instrument: 


\begin{tabular}{l|l|l|l} 
Step & Temperature $\left[{ }^{\circ} \mathrm{C}\right]$ & Time & Cycles \\
\hline Denaturation & 95 & $2 \mathrm{~min}$ & - \\
\cline { 3 - 4 } & 95 & $15 \mathrm{sec}$ & \\
\hline Annealing & 60 & $10 \mathrm{sec}$ & \multirow{2}{*}{40} \\
\hline Elongation & 72 & $20 \mathrm{sec}$ & \\
\hline Denaturation & 95 & $10 \mathrm{~min}$ & - \\
\hline Melting curve & $95.5-60$ & $10 \mathrm{sec}$ & -
\end{tabular}

Calculation of the threshold cycle (CT) and quantification of DNA was automatically performed by the system software iQ5.

\subsubsection{Immunocytochemistry}

The expression of specific markers in different cell types was investigated on the protein level by immunofluorescence analysis. For this purpose, cells were cultured on respective dishes containing glass cover slips. The cells were washed three times with DPBS and fixed with $4 \%$ PFA for $20 \mathrm{~min}$ at room temperature. Following fixation, the cells were washed three times with DPBS and blocked in $1 \%$ BSA/DPBS for at least $12 \mathrm{~h}$ at $4^{\circ} \mathrm{C}$. The cells were treated with $0.1 \%$ Triton $\mathrm{X}-100$ for $10 \mathrm{~min}$ at room temperature for permeabilization and washed three times with DPBS, except for staining of membrane-locating antigenes. The primary antibody was added to the cells and incubated at $4^{\circ} \mathrm{C}$ over-night in a humid chamber. Subsequently to three washing steps with DPBS, the secondary antibody was added to the cells and incubated at $37^{\circ} \mathrm{C}$ for $1 \mathrm{~h}$ in a humid chamber. The cells were washed three times with DPBS and stained with DAPI for $10 \mathrm{~min}$ in the dark at room temperature. Finally, the cells were washed two times with DPBS and once with distilled water, mounted onto glass slides using Vectashield Mounting Medium (Liniaris Biologische Produkte $\mathrm{GmbH}$ ) and sealed with nail polish. The slides were stored at $4^{\circ} \mathrm{C}$ and analyzed using a Zeiss Axio Observer.Z1 microscope and a Zeiss AxioCam MRm 1.4MP camera.

\subsubsection{Western blot analysis}

\subsubsection{Protein isolation}

Stored cell samples (see chapter 3.1.9) were resuspended on ice in 80-120 $\mu \mathrm{L}$ protein lysis buffer, depending on the sample size. After incubation for $10 \mathrm{~min}$ on ice, the lysates were vortexed and centrifuged for $5 \mathrm{~min}$ at $5000 \mathrm{rpm}$ and $4^{\circ} \mathrm{C}$. The supernatant was transferred into a fresh conical $1.5 \mathrm{~mL}$ tube and the protein concentration was assessed. Therefore, the 
BCA Protein Assay Kit (Pierce) was used. Samples were diluted 1:20 and the working solution was prepared by adding $4 \mu \mathrm{L}$ solution $B$ to $196 \mu \mathrm{L}$ solution A for one well of a 96-well plate. $25 \mu \mathrm{L}$ samples and provided standards were transferred in triplicates onto a 96-well plate and $200 \mu \mathrm{L}$ working solution was added. After incubation for $30 \mathrm{~min}$ at $37^{\circ} \mathrm{C}$, the protein concentration was assessed photospectroscopically at $562 \mathrm{~nm}$. Protein lysates were stored at $-80^{\circ} \mathrm{C}$ until use.

\subsubsection{SDS-polyacrylamide electrophoresis}

In order to separate proteins from each other SDS-polyacrylamide gels were made, consisting of a $15 \%$ separating gel, an $8 \%$ separating gel and a stacking gel, from bottom to top, and loaded into a respective tank containing running buffer. Protein lysates were thawed on ice and diluted in DPBS and 5x SDS sample buffer to receive a concentration of $2 \mu \mathrm{g} / \mu \mathrm{L}$ protein and $1 \times$ SDS sample buffer. The prepared lysates were incubated at $37^{\circ} \mathrm{C}$ under shaking at 300 rpm for $5 \mathrm{~min}$ and $15 \mu \mathrm{L}$ lysate and $7 \mu \mathrm{L}$ protein ladder were loaded into one gel well each. The protein separation was done using $30 \mathrm{~mA}$ current and constant voltage.

\subsubsection{Protein transfer and detection}

The proteins were transferred from the SDS-polyacrylamide gel onto a PVDF membrane using the semi-dry Trans-Blot Turbo Transfer System (Bio-Rad). Therefore, the protein containing gel was equilibrated in anode buffer for $5 \mathrm{~min}$. The PVDF membrane was activated in methanol for $2 \mathrm{~min}$, washed in $\mathrm{H}_{2} \mathrm{O}$ for $10 \mathrm{~min}$ and equilibrated in cathode buffer for 5 $\min$. Subsequently, three cathode buffer-soaked whatman paper, the membrane, the gel and three anode buffer-soaked whatman paper were stacked in a blotting cassette. The transfer was performed for $15 \mathrm{~min}$ at $2.5 \mathrm{~A}$ and $25 \mathrm{~V}$. To assess the quality of transfer and to fix proteins, the membrane was incubated for $10 \mathrm{~min}$ in Ponceau Red solution and washed with $\mathrm{H}_{2} \mathrm{O}$ until protein bands were visible. The membrane was washed for $10 \mathrm{~min}$ in TBST and blocked for $1 \mathrm{~h}$ in $5 \%$ milk/TBST at room temperature with mild shaking. Subsequently the membrane was incubated in the first antibody solution overnight at $4^{\circ} \mathrm{C}$ and washed three times with TBST for $10 \mathrm{~min}$. Second antibody solution was applied for $1 \mathrm{~h}$ at room temperature before washing three times with TBST. Afterwards, immobilon western chemiluminescent HPR substrate (Merck Millipore) was used to visualize antibody labeled proteins.

\subsubsection{Amplex Red Hydrogen Peroxide Assay}

The Amplex Red Hydrogen Peroxide/Peroxidase Assay Kit (Thermo Fisher Scientific) was used to assess the amount of $\mathrm{H}_{2} \mathrm{O}_{2}$ in the supernatant of iPSC-CMs. The assay is based on the colorless substrate Amplex Red which reacts to fluorescent resorufin in combination with horseradish peroxidase. Standards of $0.1,0.2,0.5,1$ and $2 \mu \mathrm{M} \mathrm{H}_{2} \mathrm{O}_{2}$ were prepared and 
duplicates of $50 \mu \mathrm{L}$ were transferred into a black 96-well plate. $1 \times 10^{5}$ iPSC-CMs were cultured in a well of a 12-well culture plate for one week. The cells were washed two times with DPBS and incubated in $320 \mu \mathrm{L}$ Amplex Red working solution 1 for $30 \mathrm{~min}$ at $37^{\circ} \mathrm{C}$. Immediately after treating the cells, $50 \mu \mathrm{L}$ Amplex Red working solution 2 was added to each $\mathrm{H}_{2} \mathrm{O}_{2}$ standard and also incubated for $30 \mathrm{~min}$ at $37^{\circ} \mathrm{C}$. After transferring the cell supernatant into a $1.5 \mathrm{~mL}$ conical tube, triplicates of $100 \mu \mathrm{L}$ were placed into the 96 well plate. Finally, fluorescence was measured at $540 \mathrm{~nm}$ excitation and $620 \mathrm{~nm}$ emission with a lamp energy of 5000 for $0.1 \mathrm{sec}$ per well, using a Berthold Mithras LB 940 microplate reader. The standards were used to quantify $\mathrm{H}_{2} \mathrm{O}_{2}$ concentration in samples.

\subsubsection{Plasmid isolation from E. coli culture}

The NucleoBond Xtra Maxi Plus EF Kit (Macherey-Nagel) was used to isolate DNA plasmids. A starter E. coli culture of $2 \mathrm{~mL}$ LB-medium containing an appropriate antibiotic was inoculated with a glycerol stock and incubated for $8 \mathrm{~h}$. The starter culture was added to $200 \mathrm{~mL}$ LBmedium and incubated overnight at $37^{\circ} \mathrm{C}$ with mild shaking. The E. coli were harvested by centrifugation at $6000 \times \mathrm{g}$ for $15 \mathrm{~min}$ at $4^{\circ} \mathrm{C}$ and resuspended in $12 \mathrm{~mL}$ buffer RES. $12 \mathrm{~mL}$ buffer LYS was added, inverted five times and incubated at room temperature for 5 min during which the NucleoBond Xtra column and filter were equilibrated with $25 \mathrm{~mL}$ buffer EQU. After addition of $12 \mathrm{~mL}$ buffer NEU, the lysate was immediately inverted three times and transferred onto the prepared column. $15 \mathrm{~mL}$ buffer EQU was applied and the filter removed. The column was washed with $15 \mathrm{~mL}$ buffer WASH and the DNA was eluted into a conical tube with $15 \mathrm{~mL}$ buffer ELU. To concentrate and desalt the plasmid DNA, $10.5 \mathrm{~mL}$ isopropanol was added and incubated for $2 \mathrm{~min}$. Using a $30 \mathrm{~mL}$ syringe, the precipitate was loaded onto the NucloBond Finalizer and the flow-through was discarded. After washing the Finalizer with $4 \mathrm{~mL} 70 \%$ ethanol, it was dried by pressing air through it at least six times until no more ethanol was leaking out. Plasmid DNA was eluted with $800 \mu \mathrm{L}$ TE buffer and the concentration was measured photospectroscopically.

\subsection{8 roGFP2-based biosensor analysis}

The culture medium of roGFP2-Orp1 or Grx1-roGFP2 expressing iPSC-CMs (see chapter 3.1.12) was replaced with $100 \mu \mathrm{L}$ tyrode's solution per well. Fluorescence was measured using a Berthold LB 943 Mithras $^{2}$ microplate reader with following program: 


\begin{tabular}{|l|l|}
\hline Parameter & Detail \\
\hline Temperature & $37^{\circ} \mathrm{C}$ \\
\hline Detector position & Bottom read \\
\hline Label 1 & $\begin{array}{l}\text { Ex: } 405 \mathrm{~nm} / \text { Em: } 535 \mathrm{~nm} \\
\text { counting time: } 1 \mathrm{sec} \\
\text { lamp energy: } 70 \%\end{array}$ \\
\hline Label 2 & $\begin{array}{l}\text { Ex: } 485 \mathrm{~nm} / \mathrm{Em}: 535 \mathrm{~nm} \\
\text { counting time: 0.5 sec } \\
\text { lamp energy: } 40 \%\end{array}$ \\
\hline
\end{tabular}

One repeat comprised of a label 1 measurement followed immediately by a label 2 measurement. Ten repeats were measured to receive a baseline and sextuplicats were used for each condition. Subsequently, $10 \mu \mathrm{L} 5.5 \mathrm{mM}$ diamide and $10 \mu \mathrm{L} 110 \mathrm{mM}$ DTT was automatically injected to each three wells per conditions, resulting in final concentrations of $500 \mu \mathrm{M}$ diamide and 10mM DTT. The triplicates of fully oxidized (diamide) and fully reduced (DTT) cells were then measured with ten more repeats to obtain the new baselines.

The mean of fluorescence intensities for each label was calculated so that following six sets of values obtained: Intensities of starting baseline (1405 and 1485), intensities of fully reduced baseline ( $1405_{\text {Red }}$ and $1485_{\text {Red }}$ ) and intensities of fully oxidized baseline (1405 ox and 14850x). Using Equation 2, the oxidation degree (OxD) of roGFP2 was obtained (Meyer and Dick, 2010):

$$
O x D_{\text {roGFP } 2}=\frac{I 405 \cdot I 485_{\mathrm{Red}}-I 405_{\mathrm{Red}} \cdot I 485}{I 405 \cdot I 485_{\mathrm{red}}-I 405 \cdot I 485_{\mathrm{Ox}}+I 405_{\mathrm{Ox}} \cdot I 485-I 405_{\mathrm{red}} \cdot I 485} .
$$

Using the Nernst Equation, the redox potential of $\mathrm{GSH}\left(\mathrm{E}_{\mathrm{GSH}}\right)$ was calculated (Equation 3), assuming that $\mathrm{E}_{\text {roGPP2 }}=\mathrm{E}_{\mathrm{GSH}}$ with the midpoint potential of roGFP2 $\mathrm{E}_{\text {roGP2 }}^{\circ}$ being $-280 \mathrm{mV}$. In the equation, $R$ is the gas constant, $T$ the absolute temperature and $F$ the Faraday constant.

$$
E_{G S H}=E_{\text {roGFP } 2}=E_{\text {roGFP2 }}^{\circ,}-\frac{R T}{2 F} \ln \left(\frac{1-O x D_{\text {roGFP } 2}}{O x D_{\text {roGFP } 2}}\right) .
$$

\subsubsection{Annexin $\mathrm{V}$ affinity assay and flow cytometry}

To analyze apoptosis, the APC-Annexin V Apoptosis Detection Kit with PI (BioLegend) was used. For this purpose, $1.5 \times 10^{5}$ iPSC-CMs were cultured in a well of a 6-well plate and the supernatant was transferred into a conical tube prepared with $500 \mu \mathrm{L}$ FCS. The cells were treated with $0.25 \%$ Trypsin/EDTA for $5 \mathrm{~min}$, singularized by pipetting and transferred into to conical tube. Afterwards, the cells were washed three times by centrifugation at $200 \times \mathrm{g}$ for 3 
min and subsequent resuspension in $1 \mathrm{~mL}$ PBS. After the last centrifugation step, cells were resuspended in $500 \mu \mathrm{L}$ Annexin $\mathrm{V}$ binding buffer and $100 \mu \mathrm{L}$ suspension was transferred into each four cytometry tubes. Following staining combinations were prepared by addition of 5 $\mu \mathrm{L}$ APC-Annexin $\mathrm{V}$ and $10 \mu \mathrm{L}$ propidium iodide (PI): unstained, only APC-Annexin V, only PI, APC-Annexin V / PI double staining. The suspensions were incubated for $15 \mathrm{~min}$ in the dark and $400 \mu \mathrm{L}$ Annexin $\mathrm{V}$ binding buffer was added to each tube. Finally, the stained cells were measured using a BD FACSCanto II (BD Biosciences). Unstained and single stainings were used to set up analysis grids to distinguish populations.

\subsubsection{Calcium imaging}

Cytosolic $\mathrm{Ca}^{2+}$ was visualized in iPSC-CMs with Fluo-4, which exhibits increased fluorescence upon $\mathrm{Ca}^{2+}$ binding. For this purpose, $2.5 \times 10^{5}$ cells were plated in a well of a 6 -well plate containing a round $20 \mathrm{~mm}$ glass cover-slip and cultured for one week. The cover-slip was mounted onto the measuring chamber and the cells were incubated in $400 \mu \mathrm{L}$ Fluo- 4 staining solution for $30 \mathrm{~min}$ in the dark before they were washed two times with tyrode's solution and finally covered with $800 \mu \mathrm{L}$ tryrodes solution. The measuring chamber was mounted onto a LSM 720 confocal microscope (Zeiss) and the software Zen 2009 was used for measurement. The microscope was set up with following parameters: laser $488 \mathrm{~nm}$, excitation $0.5-2 \%$, pinhole $6 \mathrm{AU}$, gain 700, offset 0 , line scan mode, zoom 3, 12 bit unidirectional, 512 pixel, maximal speed, 20.000 cycles, no delay. The iPSC-CMs were paced during measurement at $0.25 \mathrm{~Hz}$ with $18 \mathrm{~V}$ and $3 \mathrm{~ms}$ duration. Measuring lines were placed in the cytoplasm of iPSC-CMs without crossing cell organelles.

The fluorescence intensities of the obtained linescans were plotted and normalized with the software FIJI and smoothed with the software Prism6 (GraphPad). Subsequently, $\mathrm{Ca}^{2+}$ transients were automatically analyzed with the software Labchart (ADInstruments). The transient rise time is the time interval from transient start to transient peak, whereas the decay time was defined as time interval from peak to the time point where half of the fluorescence signal is diminished. To calculate the peak amplitude the baseline fluorescence intensity was subtracted from the peak fluorescence intensity and subsequently divided by the baseline fluorescence intensity (see Figure 23).

\subsection{In vivo teratoma formation and histological analysis}

iPSCs were cultured on three 6-cm culture dishes with MEFs and digested as described above (see chapter 3.1.4). The cells were pooled, centrifuged at $200 \mathrm{xg}$ for $3 \mathrm{~min}$ and resuspended in $300 \mu \mathrm{L}$ DPBS. Subsequently, they were injected subcutaneously into immunodeficient mice by Prof. Dr. med. Ralf Dressel from the Institute for Cellular and Molecular Immunology, University Medical Center Göttingen. About three month later, the mice were killed and the 
tumors collected. After fixing the tumors in phosphate buffered formalin $(\mathrm{pH}$ 7) for $4 \mathrm{~h}$ at room temperature or over-night at $4^{\circ} \mathrm{C}$, they were washed in $\mathrm{H}_{2} \mathrm{O}$, dehydrated and paraffinized with a Benchtop Tissue Processor 1020 (Leica Biosystems). Using a tissue embedding system (Leica Biosystems), the tumors were embedded into paraffin and $6 \mu \mathrm{m}$ sections were attached onto glass specimen holders. Hematoxilin and eosin stainings $(\mathrm{H}+\mathrm{E})$ were produced by the Department of Pathology, University Medical Center Göttingen.

\subsection{Analysis of $\alpha$-actinin regularity}

$\alpha$-actinin was visualized in iPSC-CMs by immunofluorescence staining as described above (3.2.4). Using the software FIJI, the integrated plugin "Tubness" was applied to the obtained images, which emphasizes tubular structures such as the sarcomeric cytoskeleton and reduces artifacts. Subsequently, the fast Fourier transform algorithm was applied and resulting frequency domains were radially integrated with the open source plugin "Radial Profile Plot" from Paul Baggethun (https://imagej.nih.gov/ij/plugins/radial-profile.html as of October 19, 2017). The software LabChart (BDInstruments) was used to automatically analyze the relative amplitude of the first peak in the intensity profile. This amplitude was used to assess the regularity of $\alpha$-actinin striations in iPSC-CMs.

\subsection{Statistical analyses}

Data is depicted as mean + standard error of mean (SEM). Two-tailed students t-test was applied to data sets consisting of two groups. Two-way analysis of variance (ANOVA) was applied to data sets with more than two groups. Multiple comparisons between control and patient group were corrected according to Sidak, whereas multiple comparisons of DOX treatment conditions with basal condition were corrected according to Dunnett. Statistical significance is depicted as $* p<0.05, * * p<0.01, * * * p<0.001$. Statistical analysis was performed with Prism6/7 (GraphPad). 


\section{$4 \quad$ Results}

\subsection{Recruitment of donors}

For this study, five donors were recruited who were participants of the "rituximab with CHOP over age 60 years" (RICOVER60) trial, which was performed from July 2000 until June 2005 and is registered on www.clinicaltrials.gov as NCT00052936. The RICOVER60 trial aimed to compare the outcome in elderly patients with diffuse large-B cell lymphoma after treatment with either CHOP-14 (cyclophosphamide, doxorubicin, vincristine and prednisolone in two weeks intervals) or CHOP-14 with addition of rituximab (Pfreundschuh et al., 2008). In the work of Reichwagen et al. the group of Wojinowski used the data from the RICOVER60 trial to analyze associations of SNPs in the genes MRP1, MRP2, CYBA, NCF4, RAC2 and SLC28A3 with ACT (Reichwagen et al., 2015). They found an accumulation of SNPs rs4673 (genotype CT and TT) of CYBA and of rs13058338 (TA and AA) of RAC2 among ACT cases. The investigated genes were selected on the basis of previous findings in the NHL-B1/B2 study from the same group. Here, the aforementioned SNPs were also found to be associated with ACT (Wojnowski et al., 2005).

The classification of RICOVER60 trial participants into ACT patients and controls was done by Reichwagen et al. and was based on records of physical examinations that were taken during therapy and follow-up investigations (Reichwagen et al., 2015). Symptoms that resulted in ACT classification included "reduced ejection fraction or fractional shortening, arrhythmia, dyspnea, heart failure diagnosis and / or treatment, cardiomyopathy, coronary heart disease including myocardial infarction and death for cardiac reasons". Starting 2003 and subsequent to the classification of ACT patients and controls, blood samples were obtained for genotyping. To validate the classification, the blood samples were also used to assess the NTproBNP concentration, which is a marker for congestive heart failure.

According to the classification of RICOVER60 participants by Reichwagen et al. and to their genotypes, three patients could be recruited for our study who suffered from chronic ACT and have the predisposing alleles of SNPs in CYBA and RAC2 (Table 1 and 2). Furthermore, two controls were recruited who did not develop cardiac symptoms after chemotherapy and did not possess predisposing alleles of SNPs in CYBA and RAC2. Neither the ACT patients nor the controls had cardiac conditions before chemotherapy. All available information concerning the donors are summed up in Table 1. 
4 Results

Table 1: Overview of recruited donors from the RICOVER60 trial.

\begin{tabular}{|c|c|c|c|c|c|}
\hline & Patient 1 & Patient 2 & Patient 3 & Control 1 & Control 2 \\
\hline Sex & Male & Male & Female & Male & Male \\
\hline Age in 2007 & 69 & 71 & 66 & 66 & 68 \\
\hline $\begin{array}{l}\text { DOX dose } \\
\left.\text { [mg / } \mathrm{m}^{2}\right] \text {; median of } \\
\text { patients and controls }\end{array}$ & \multicolumn{3}{|c|}{309} & \multicolumn{2}{|c|}{318} \\
\hline Developed ACT & $\begin{array}{c}\text { Yes } \\
\text { (chronic) }\end{array}$ & $\begin{array}{c}\text { Yes } \\
\text { (chronic) }\end{array}$ & $\begin{array}{c}\text { Yes } \\
\text { (chronic) }\end{array}$ & No & No \\
\hline $\begin{array}{l}\text { NT-proBNP } \\
\text { concentration by the } \\
\text { time of genotyping } \\
\text { (2007) [pg / mL] }\end{array}$ & 220.1 & 160.5 & 500.9 & 11.91 & 149.9 \\
\hline $\begin{array}{l}\text { LVEF at the time of } \\
\text { biopsy extraction } \\
\text { (2014) }\end{array}$ & $\begin{array}{l}\text { Not } \\
\text { available }\end{array}$ & са. $45 \%$ & $45-50 \%$ & $\begin{array}{l}\text { Not } \\
\text { available }\end{array}$ & $\begin{array}{l}\text { Not } \\
\text { available }\end{array}$ \\
\hline
\end{tabular}

Table 2: Genotypes of donors at ACT-associated SNPs. P: patient; C: Control; underlined genotypes are associated with ACT.

\begin{tabular}{|c|c|c|c|c|c|c|c|c|c|}
\hline \multirow{2}{*}{ Gene } & \multirow{2}{*}{ SNP } & \multirow{2}{*}{$\begin{array}{c}\text { Possible } \\
\text { Genotypes }\end{array}$} & \multirow{2}{*}{$\begin{array}{l}\text { Nucleotide } \\
\text { position }\end{array}$} & \multirow{2}{*}{ Effect of SNP } & \multicolumn{5}{|c|}{ Genotype of donors } \\
\hline & & & & & P1 & $\mathbf{P 2}$ & P3 & C1 & C2 \\
\hline CYBA & rs4673 & $\mathrm{CC} / \underline{\mathrm{CT} / \mathrm{TT}}$ & $242 \mathrm{C}>\mathrm{T}$ & $\begin{array}{l}\text { Amino acid } \\
\text { substitution } \\
\text { (H72Y) }\end{array}$ & CT & CT & CT & $\mathrm{CC}$ & $\mathrm{CC}$ \\
\hline RAC2 & rs13058338 & TT/TA/AA & $7508 \mathrm{~T}>\mathrm{A}$ & $\begin{array}{c}\text { No amino acid } \\
\text { substitution; SNP } \\
\text { in intronic region }\end{array}$ & AA & TA & TA & TT & TT \\
\hline
\end{tabular}

Skin biopsies were taken from the donors in respective medical centers near their residences and delivered to the University Medical Center Göttingen. Here, the biopsies were incubated under cell culture conditions, causing dermal fibroblasts to proliferate and grow out of the biopsy in about one week. The dermal fibroblasts were cultured and used for the generation of hiPSCs and further analysis. To confirm the presence of SNPS in CYBA and RAC2, genomic DNA was isolated from dermal fibroblasts and used for sequencing (Figure 9). The expected alleles (see table 2) were found in all patients and controls. Sequencing of DNA isolated from hiPSCs confirmed the presence of the alleles. 
CYBA
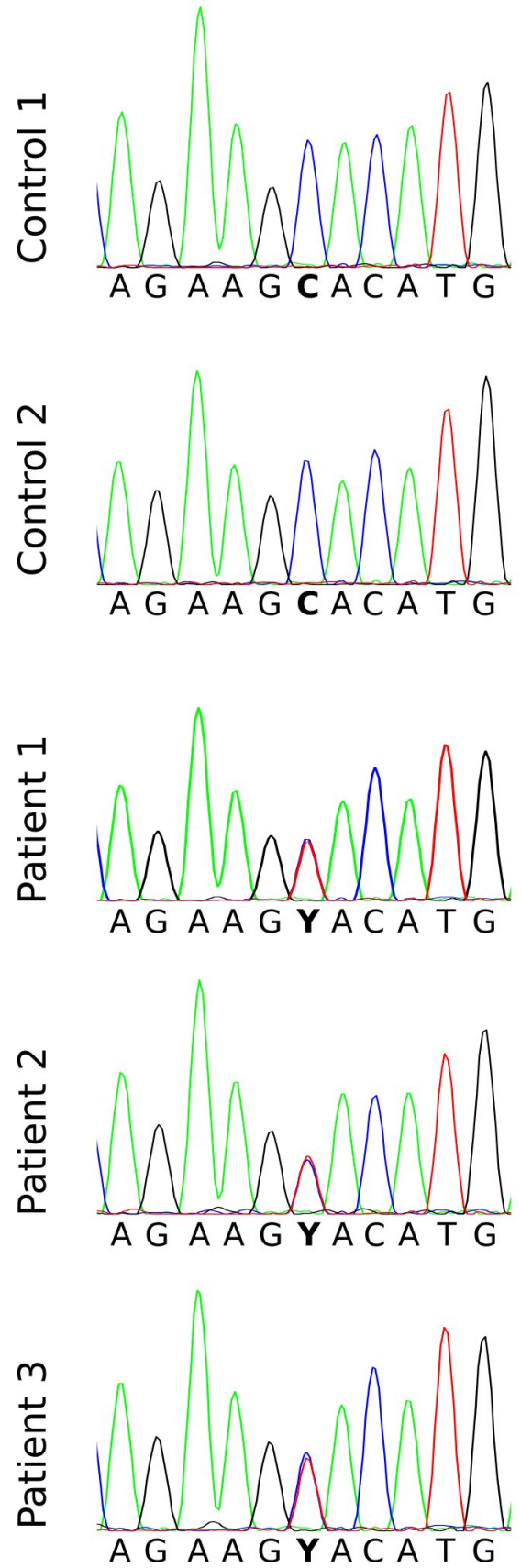

RAC2
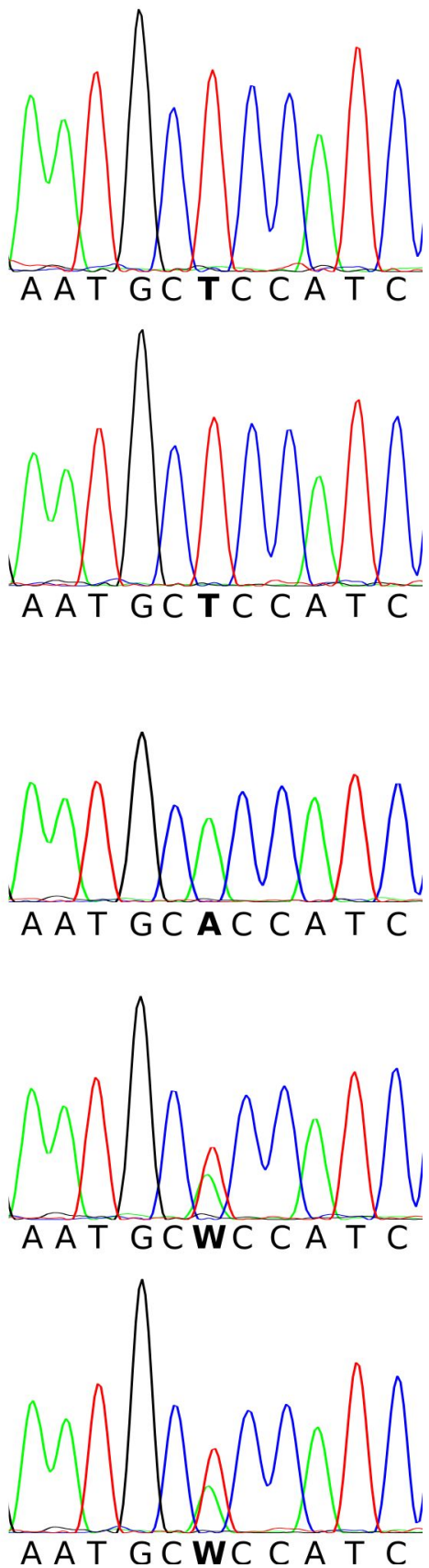

Figure 9: Verification of ACT-associated SNPs in CYBA and RAC2. ACT-associated alleles of SNPs in NADPH oxidase subunit encoding genes CYBA (rs4673) and RAC2 (rs13058338) were confirmed in all recruited ACT patients. None were found in controls. Sequencing chromatograms are depicted and respective SNPs are marked with bold text. 


\subsection{Generation and characterization of hiPSCs}

\subsubsection{Generation of hiPSCs}

To generate hiPSCs from primary dermal fibroblasts, the integration-free sendai virus system was used, which contains the four reprogramming factors OCT4, KLF4, SOX2 and C-MYC on three separate vectors. Fibroblasts displayed a typical elongated morphology and were passaged only once to ensure high reprogramming efficiency (Figure $10 \mathrm{~A}, \mathrm{D}$ ). First morphological changes of fibroblasts were notable about seven days after transduction and single hES-like colonies were mechanically selected two to three weeks after transduction. Cells from about 20 colonies per donor were cultured and seven to nine cell lines per donor were selected for cryopreservation according to their morphology and proliferation (Figure $10 \mathrm{~B}, \mathrm{E})$. The reprogramming efficiency was very high in all transduction experiments with about $7 \%$, estimated by staining for alkaline phosphatase activity. No differences in morphology of fibroblasts and iPSCs or reprogramming efficiency were observed between ACT patients and controls. Two cell lines per donor were selected for characterization and cardiomyocyte differentiation. A high alkaline phosphatase activity was detected in those cell lines, which is an early marker for pluripotency (Figure $10 \mathrm{C}, \mathrm{F}$ ).
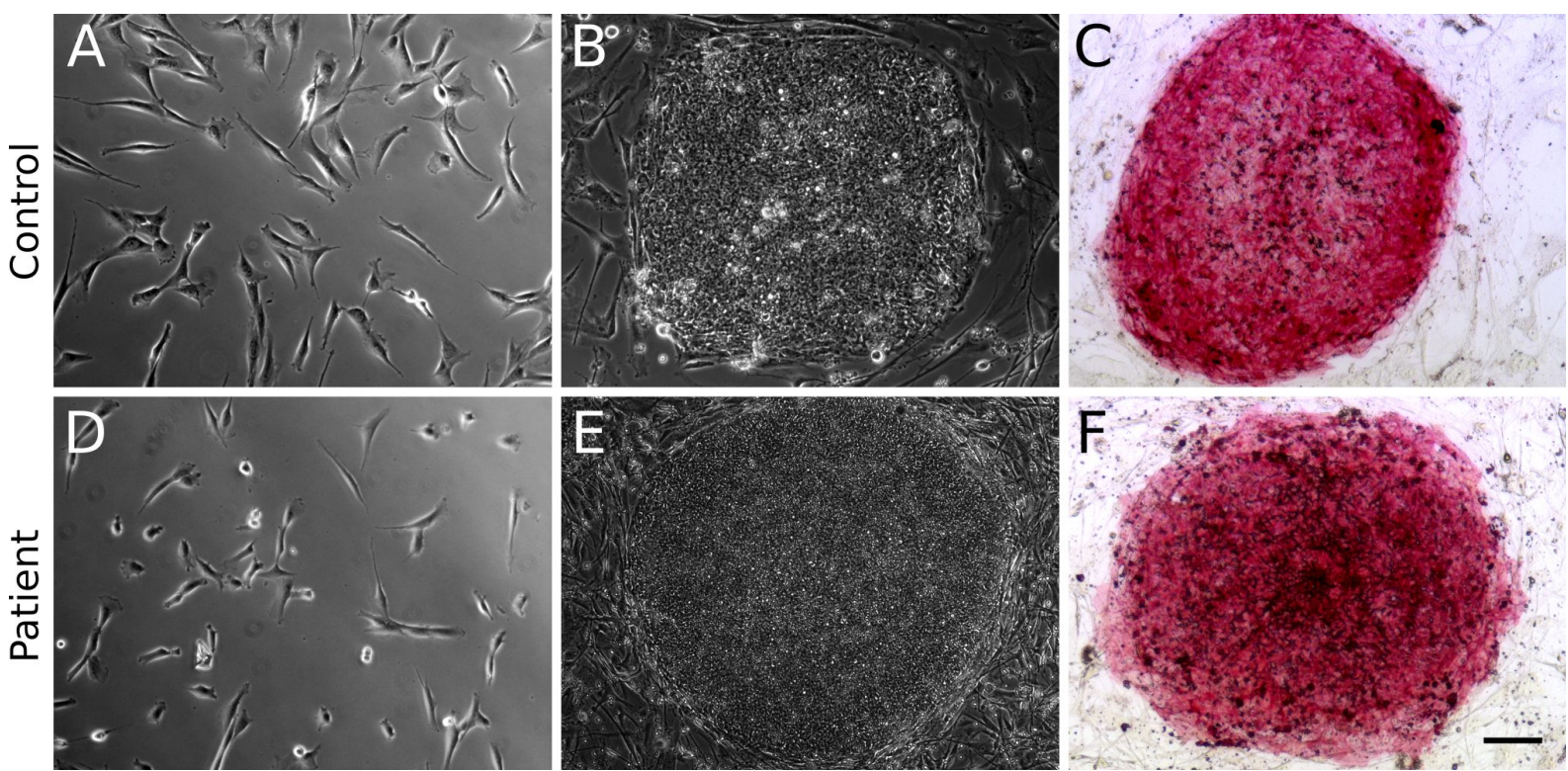

Figure 10: Generation of hiPSCs and detection of alkaline phosphatase activity. A, D: Dermal fibroblasts of controls and ACT patients. B, E: hiPSCs cocultured with MEFs. C, F: Visualization of alkaline phosphatase activity in hiPSCs (red staining). Depicted images (Control 1 and Patient 1 ) are representative for all cell lines. Scale bar: $200 \mu \mathrm{m}$.

\subsubsection{Expression of pluripotency-related genes}

Contrary to somatic cells, iPSCs express a network of genes necessary for maintaining pluripotency. To estimate the level of pluripotency of the generated iPSCs, expression of the 
pluripotency markers NANOG, OCT4, LIN28, SOX2 and GDF3 (growth differentiation factor-3) was investigated on the mRNA level by semiquantitative RT-PCR (Figure 11). For this purpose, RNA of iPSCs at passage number 9 to 15 as well as RNA of the corresponding fibroblasts was used. RNA of the control cell line FB1 was used as positive control since these cells show expression of pluripotency marker genes comparable to hES cells (Streckfuss-Bömeke et al., 2013). The housekeeping gene GAPDH was used as a reference. All investigated genes were upregulated in the iPSCS compared to their parental fibroblasts and at a similar expression level as control iPSCs. These data suggest an activation of the endogenous pluripotency network during reprogramming.

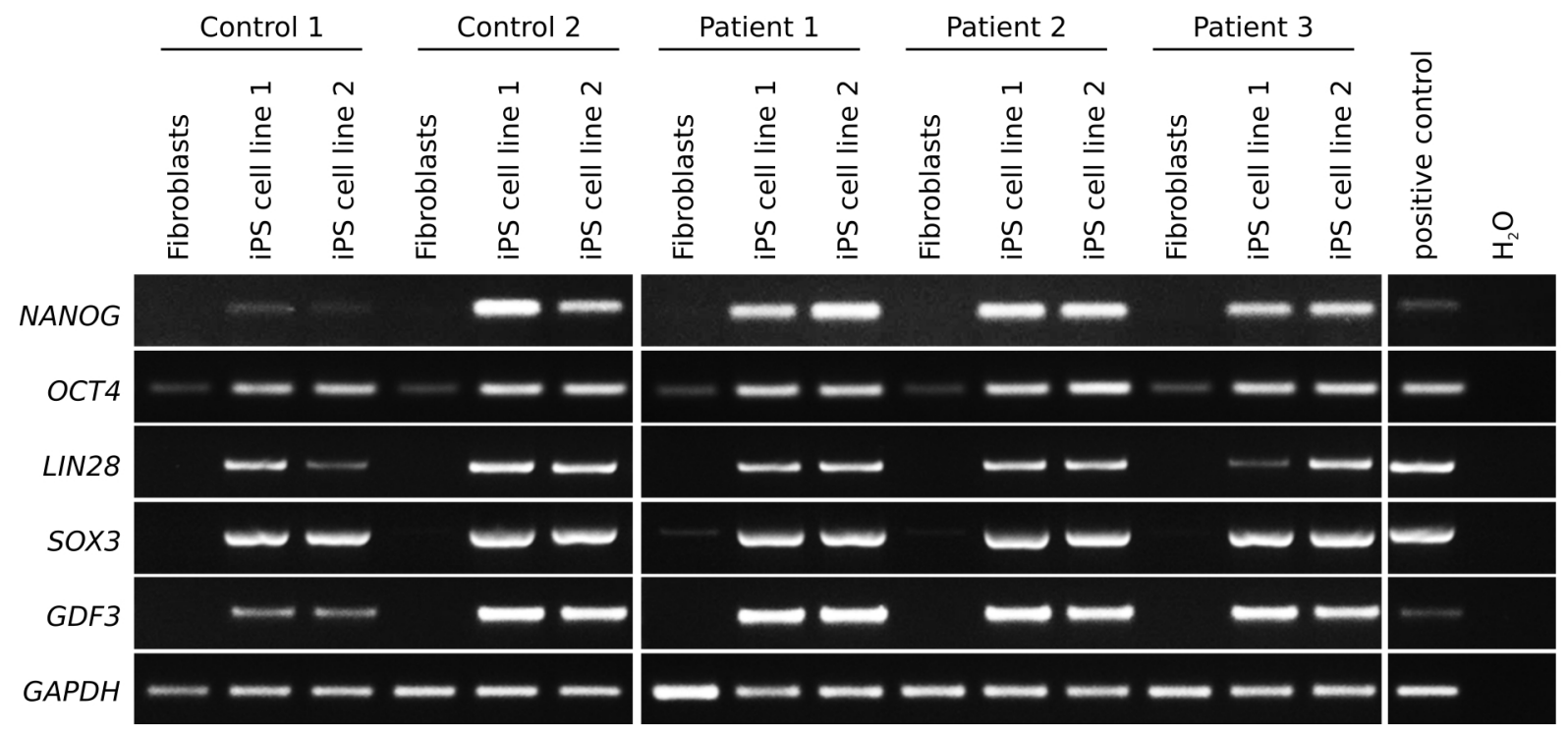

Figure 11: Expression of pluripotency-related genes on the mRNA level. Semiquantitative RT-PCR analysis confirmed expression of NANOG, OCT4, LIN28, SOX2 and GDF3 in all iPSC lines selected for characterization and in positive control iPSCs (FB1). Low or no expression was observed in fibroblasts used for reprogramming. GAPDH was used as reference gene. $\mathrm{H}_{2} \mathrm{O}$ was used as negative control.

The expression of pluripotency markers was also investigated on the protein level by immunofluorescence analysis (Figure 12). LIN28, NANOG, OCT4, SOX2, SSEA4 and TRA-1-60 were stained at passage number 7 to 15 . Specific signals were found in iPSC colonies but not in MEFs. LIN28 was located in the cytoplasm. NANOG, OCT4 and SOX2 had a distinct nuclear localization while SSEA4 and TRA-1-60 were observed in the cell membrane. No differences between cell lines from ACT patients (ACT-iPSCs) and controls (Ctrl-iPSCs) were observed. 

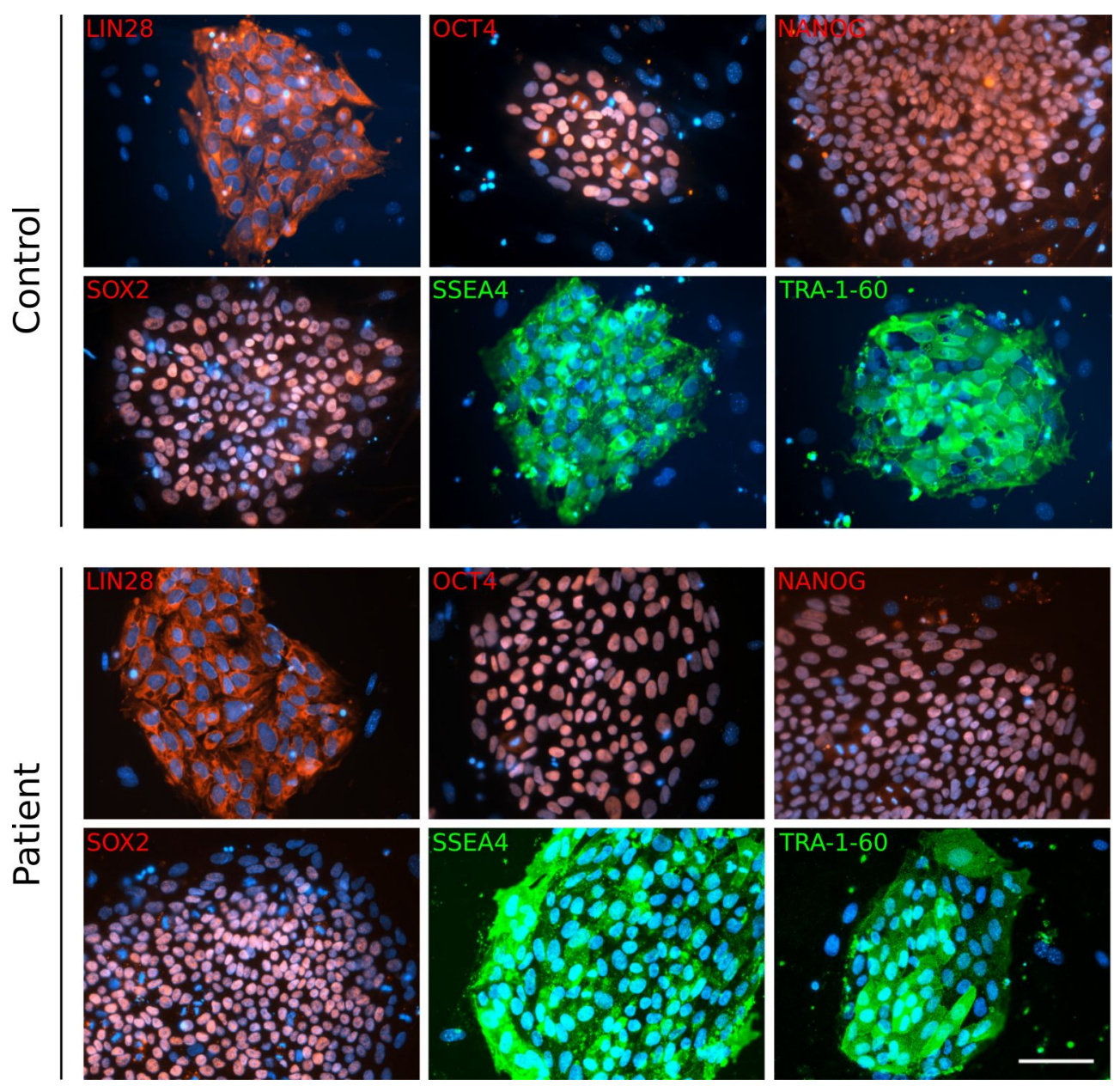

Figure 12: Expression of pluripotency-related genes on protein level. LIN28, OCT4, NANOG, SOX2, SSEA4 and TRA-1-60 were observed in generated Ctrl-iPSCs and ACT-iPSCs cocultured with MEFs by immunofluorescence staining. Nuclei were stained with DAPI (blue). Depicted images (Control 1 and Patient 3) are representative for all cell lines. Scale bar: $100 \mu \mathrm{m}$.

\subsubsection{Differentiation potential in vitro and in vivo}

The characterization of the iPSCs also included the investigation of their spontaneous differentiation potential. For this purpose, iPSCs at passage number 10-18 were spontaneously differentiated in vitro and the expression of specific markers for each germ layer was analyzed on the mRNA and protein level. The iPSCs were cultured in suspension for eight days. During this time the cells developed round-shaped embryoid bodies (EBs) with clear, smooth borders. The EBs attached to gelatine-coated cover-slips one to two days after plating at day 8. During the next 25 days, cells grew out of the EBs and developed into a large variety of cell types.

RNA was isolated at day 0 , day 8 and day 33 of in vitro differentiation and used for gene expression analyses by semiquantitative RT-PCR (Figure 13). RNA of MEFs was used as negative control. The pluripotency-related gene NANOG was expressed in undifferentiated cells and was downregulated during spontaneous differentiation in most experiments. 
Remaining NANOG expression suggests that few cells were in an undifferentiated stage at the end of differentiation. In all cell lines, the expression of endodermal $(A F P)$, mesodermal (cTNT) and ectodermal (TH) markers increased with proceeding differentiation time with the exception of AFP expression in cell line 1 of control 2 and cell line 2 of patient 2. In few experiments, germlayer markers were already expressed in undifferentiated cells.

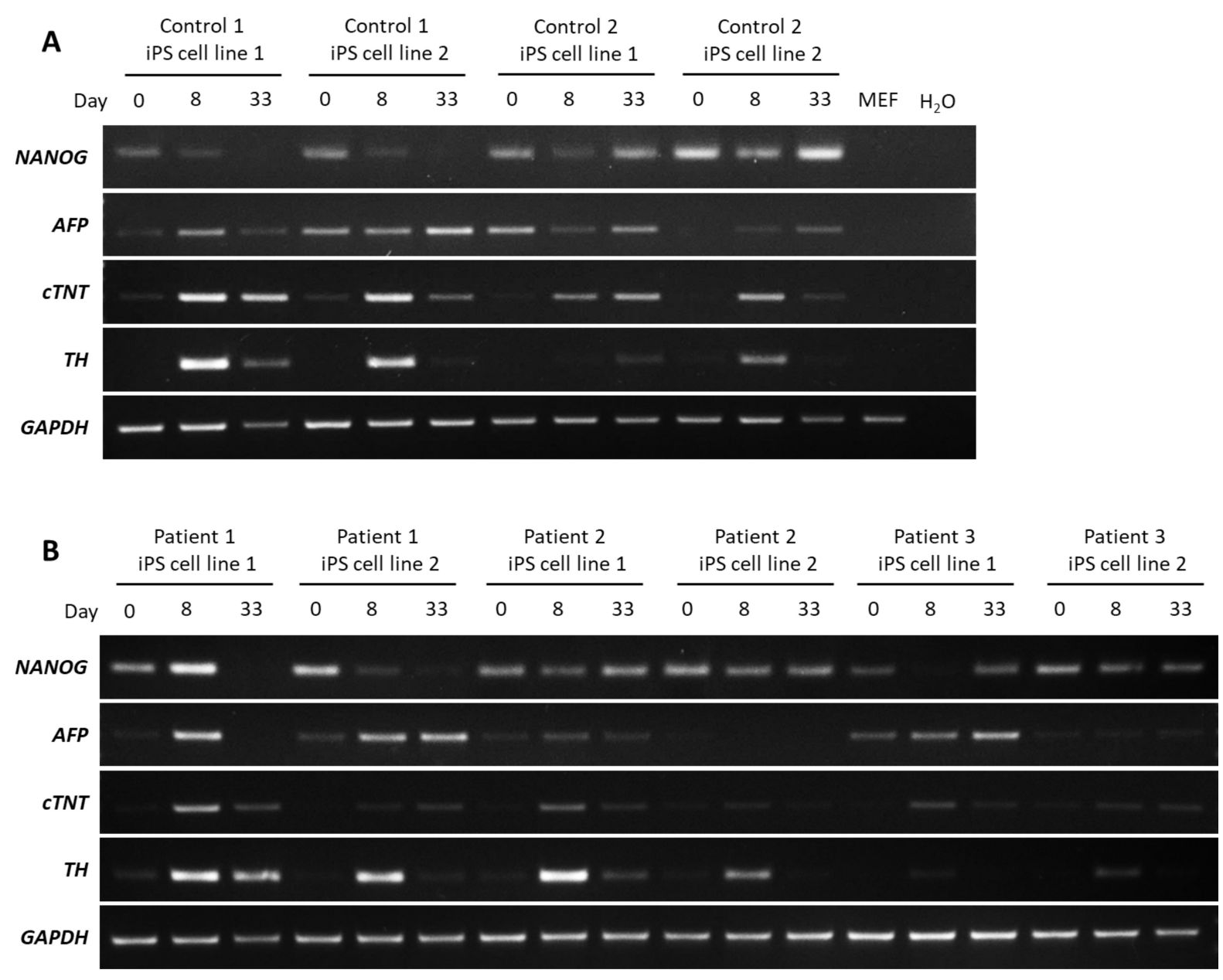

Figure 13: Expression of germ layer-specific genes on mRNA level after spontaneous differentiation of iPSCs. A, B: Expression of germ layer-specific genes AFP, CTNT and TH after prolonged spontaneous differentiation using semiquantitative RT-PCR in Ctrl- and ACT-iPSCs. Downregulation of NANOG expression indicated loss of pluripotency. GAPDH was used as reference gene. MEFs and $\mathrm{H}_{2} \mathrm{O}$ were used as negative control.

After prolonged differentiation, the cells were fixed and immunofluorescence analysis was performed. $\beta$-III-Tubulin ( $\beta$-III-Tub), $\alpha$-smooth muscle actin ( $\alpha$ SMA) and AFP were stained as representatives for the ectoderm, mesoderm and endoderm, respectively (Figure 14). At day 33 of differentiation, $\beta$-III-Tub and $\alpha$ SMA staining showed cytoskeletal structures of neuronlike and muscle-like cells, respectively. AFP was located in the cytoplasm at day 18 of differentiation. The three markers could be observed in all cell lines and no differences were found between Ctrl- and ACT-iPSCs. 

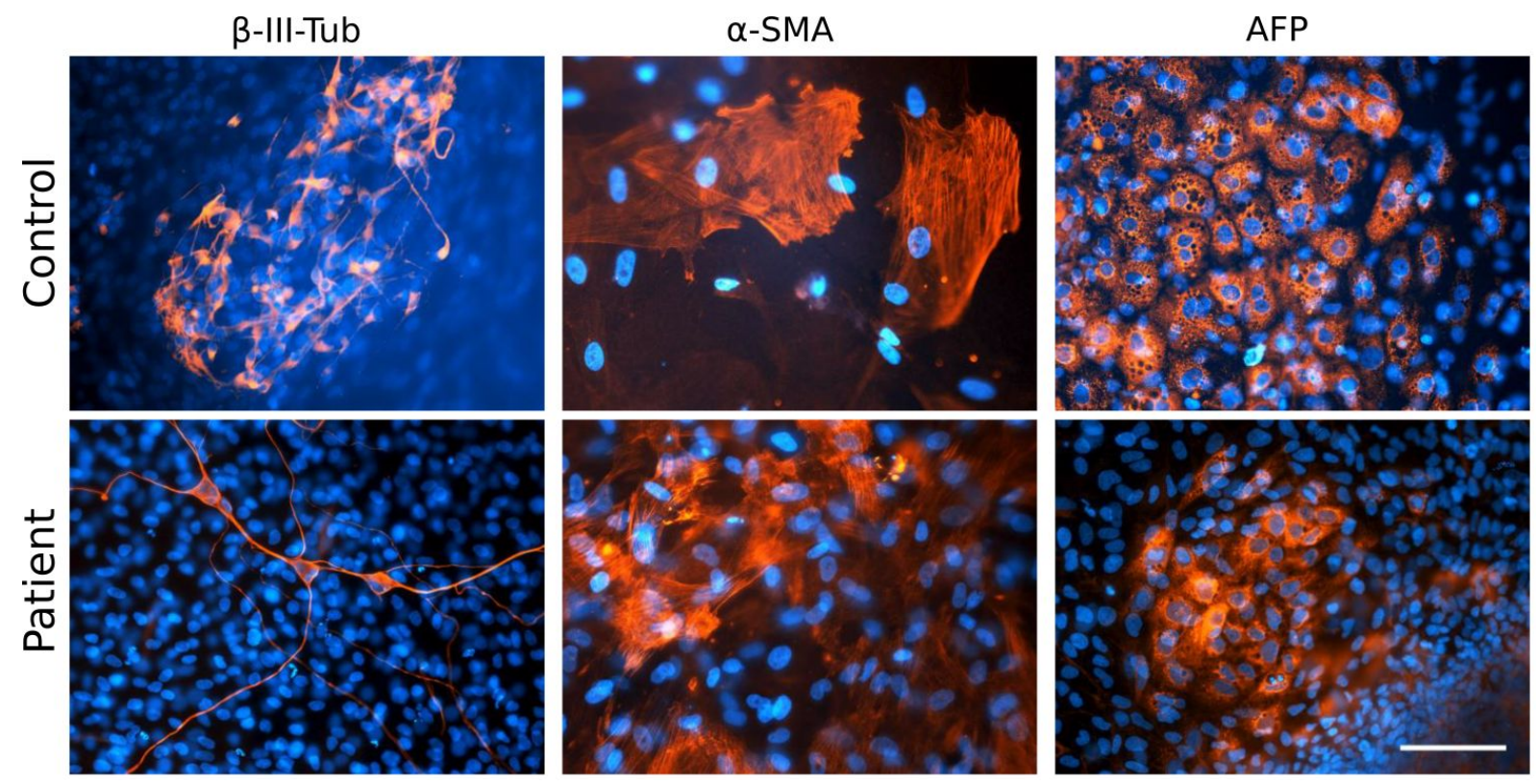

Figure 14: Expression of germ layer-specific genes after spontaneous in vitro differentiation of iPSCs on protein level. Expression of $\beta$-III-Tub, $\alpha$-SMA and AFP after spontaneous differentiation of iPSCs by immunofluorescence staining. Nuclei were stained with DAPI (blue). Depicted images (Control 1 and Patient 3) are representative for all cell lines. Scale bar: $100 \mu \mathrm{m}$.

Finally, the differentiation potential of the generated iPSCs was determined in vivo by teratoma formation in immunodeficient mice and histological analysis. The teratomas contained tissues derived from all three germ layers including neuronal rosettes (ectoderm, Figure $15 \mathrm{~A}, \mathrm{D}$ ), muscle (mesoderm, Figure $15 \mathrm{~B}, \mathrm{E}$ ) and intestinal tissue (endoderm, Figure 15 $C, F)$. Some cell lines did not develop teratomas after injection, however at least one teratoma per donor was received and analyzed. 

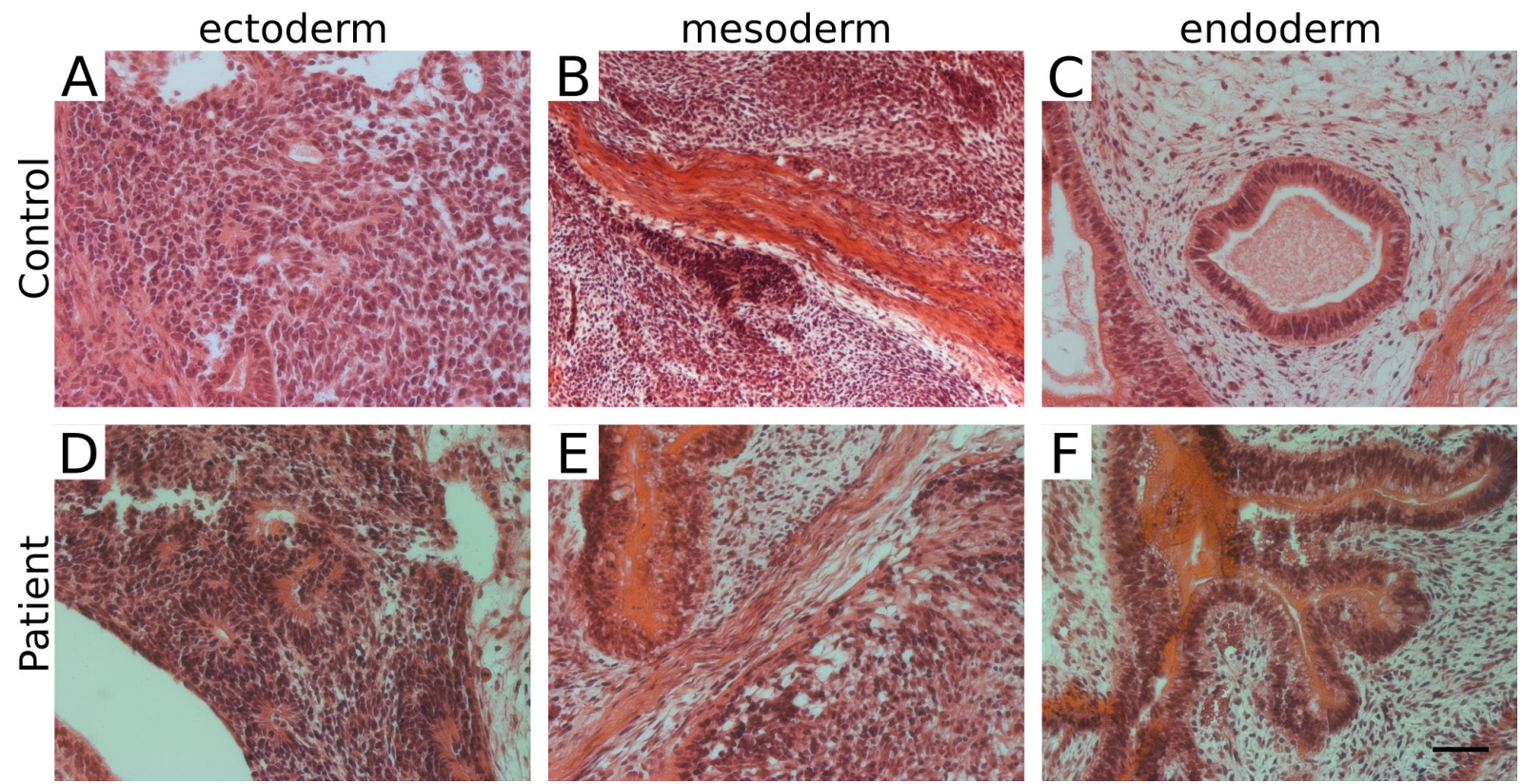

Figure 15: In vivo differentiation of generated iPSCs. Teratoma preparations were stained with $\mathrm{H}+\mathrm{E}$. A, D: Neuronal rosettes. B, E: Muscle tissue. C, F: Intestinal tissue. Depicted images (Control 1 and Patient 2) are representative for all generated teratomas. Scale bar: $100 \mu \mathrm{m}$.

The generated iPSCs were to be used as a source for CMs. Therefore, the spontaneous cardiac differentiation potential was assessed since the potential to differentiate into a specific cell type may be cell line-specific. For this purpose, EBs that developed beating areas were counted. 7-21 \% of EBs spontaneously developed beating areas with no differences between controls (15.4 $\pm 3.45 \%)$ and ACT patients (15.4 $\pm 2.51 \%)$ (Table 3 ).

Table 3: Spontaneous cardiac differentiation potential. About 30 EBs were analyzed per experiment. Cardiac differentiation potential is depicted as mean \pm SEM.

\begin{tabular}{|l|c|c|}
\hline & $\begin{array}{l}\text { Number of } \\
\text { experiments }\end{array}$ & $\begin{array}{l}\text { Cardiac differentiation } \\
\text { potential [\%] }\end{array}$ \\
\hline Control 1, cell line 1 & 2 & $14.9 \pm 3.79$ \\
\hline Control 1, cell line 2 & 2 & $18.5 \pm 0.21$ \\
\hline Control 2, cell line 1 & 1 & 11.1 \\
\hline Control 2, cell line 2 & 3 & $15 \pm 9.96$ \\
\hline Patient 1, cell line 1 & 3 & $14.3 \pm 5.27$ \\
\hline Patient 1, cell line 2 & 1 & 8.2 \\
\hline Patient 2, cell line 1 & 3 & $13.2 \pm 1.07$ \\
\hline Patient 2, cell line 2 & 3 & $20.8 \pm 9.99$ \\
\hline Patient 3, cell line 1 & 1 & 7.1 \\
\hline Patient 3, cell line 2 & 3 & $18.3 \pm 4.59$ \\
\hline
\end{tabular}




\subsection{Generation of iPSC-derived cardiomyocytes}

Using the spontaneous differentiation protocol, at most $21 \%$ of the EBs per differentiation experiment developed beating areas (Table 3 ). Since these beating areas only made up a small part of the cells developed by one EB, the CM yield gained by spontaneous differentiation was not sufficient for later analysis. To obtain a high amount and purity of $\mathrm{CMs}$, the iPSCs were directly differentiated using an activator of the canonical WNT pathway, CHIR, and an inhibitor, IWP2, at different stages of differentiation to mimic in vivo heart development. For this purpose, iPSCs were grown to $90 \%$ confluency on geltrex without feeder cells before CHIR was added. During the next days, the cells formed diverse structures and first beating areas were usually observed at day 8-15. Often, these areas were large and tissue-like. Nevertheless, smaller beating clusters, which resembled EBs, were also frequently observed. Despite using the same conditions, the amount of beating areas strongly varied between different experiments of the same cell line. Estimated visually, the CM yield was considerably larger compared with spontaneous differentiation. Furthermore, a high purity of over $90 \%$ CMs was achieved by metabolic selection with lactate, observed visually and by flow cytometry of cTNT stained cells (Figure $16 \mathrm{~A}$ ). No differences were observed between the ACT patient and control groups regarding differentiation efficiency or CM purity. 

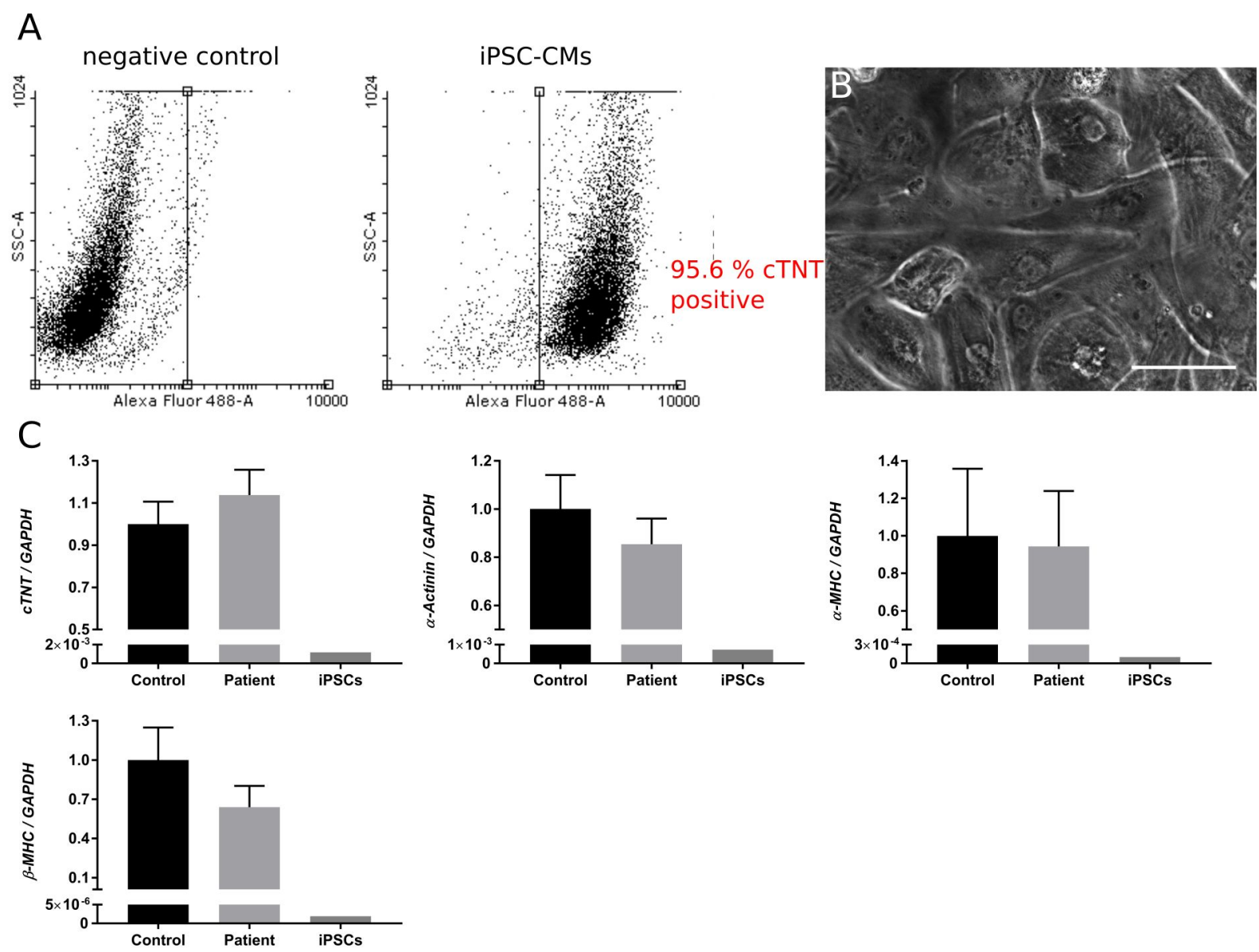

$\mathrm{D}$
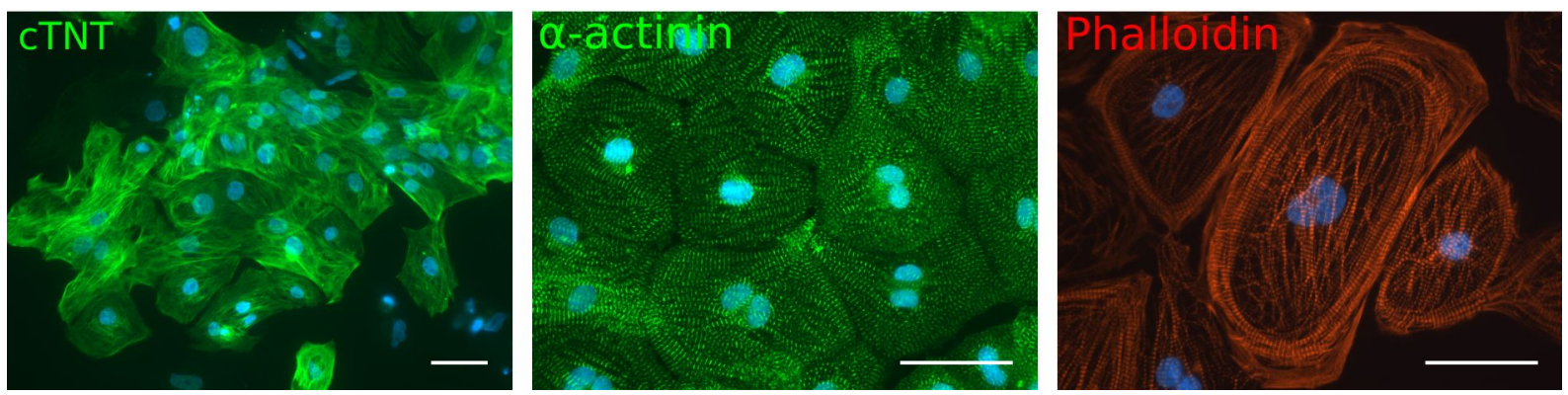

Figure 16: Characterization of iPSC-CMs. A: Flow cytometry analysis of iPSC-CMs stained against cTNT (secondary antibody was Alexa Fluor488-conjugated). Unstained iPSC-CMs were used as negative control and to setup the gate. Shown are results from iPSC-CMs from Patient 2 cell line 1. B: Morphology of iPSC-CMs. C: qRT-PCR analysis of cardiac genes in Ctrl- and ACT-iPSC-CMs and iPSCs. D: Immunofluorescence stainings of cTNT and $\alpha$-actinin. Alexa Fluor555-conjugated phalloidin was used to visualize F-actin. Nuclei were stained with DAPI (blue). Scale bars: $50 \mu \mathrm{m}$.

The two-month-old iPSC-CMs derived from ACT-iPSCs (ACT-iPSC-CMs) and Ctrl-iPSCs (CtrliPSC-CMs) had a heterogeneous morphology with varying sizes and elongated, round or multiangular shapes (Figure $16 \mathrm{~A}, \mathrm{D}$ ). They expressed the cardiac genes $\alpha$-actinin, cTNT, $\alpha$ $M H C$ and $\beta-M H C$ as detected with qRT-PCR without significant differences between the ACT patient and control groups. Expression of these genes was barely found in iPSCs (Figure 16 C). Furthermore, visualization of cTNT, $\alpha$-actinin and F-actin revealed a high organization of 
sarcomeres with clear striations along the cell. $\mathrm{Bi}$ - or multinucleated iPSC-CMs were frequently observed (Figure $16 \mathrm{D}$ ).

\subsection{Modeling ACT in hiPSC-CMs}

This work aimed to recapitulate the cardiac phenotypes of ACT patients and controls in response to DOX treatment. Therefor, two- to three-month-old iPSC-CMs were pooled and plated one week prior to the experiment to ensure comparability between tested conditions. To analyze the dose-dependency of phenotypes, DOX was added to the cell medium in concentrations of $0.1,0.25,0.5,0.75,1$ and $5 \mu \mathrm{M}$. DOX was applied for 24 hours if not stated otherwise. In some experiments the number of conditions was decreased on account of feasibility.

\subsubsection{NADPH oxidase}

The expression of NADPH oxidase subunits NOX2, NOX4, CYBA, RAC1, RAC2, NCF1, NCF2, NCF4 was analyzed by qRT-PCR (Figure 17). Despite best efforts, expression of NCF1, NCF2 and NCF4 was not detectable in iPSC-CMs but in biopsies of left ventricles of a dilated cardiomyopathy patient (see appendix, Figure 35), indicating that the designed primers are suitable for amplification in the presence of target cDNA. HPRT (hypoxanthine-guanine phosphoribosyl-transferase) was used as a reference gene for genes with low expression (see appendix, Table 5). At basal conditions, the expression of NOX2, NOX4, CYBA and RAC1 was comparable between Ctrl- and ACT-iPSC-CMs. RAC2 was expressed 1.5-fold higher in ACTiPSC-CMs, which was not significant, however. Treatment with $0.25 \mu \mathrm{M}$ DOX caused a significant downregulation of NOX2 in Ctrl-iPSC-CMs and a slight decrease in NOX4, CYBA and $R A C 2$ expression, whereas RAC1 expression was not altered. In ACT patients, on the other hand, expression of NOX2, NOX4, CYBA and RAC1 was significantly downregulated after DOX treatment, but $R A C 2$ was unaffected. 

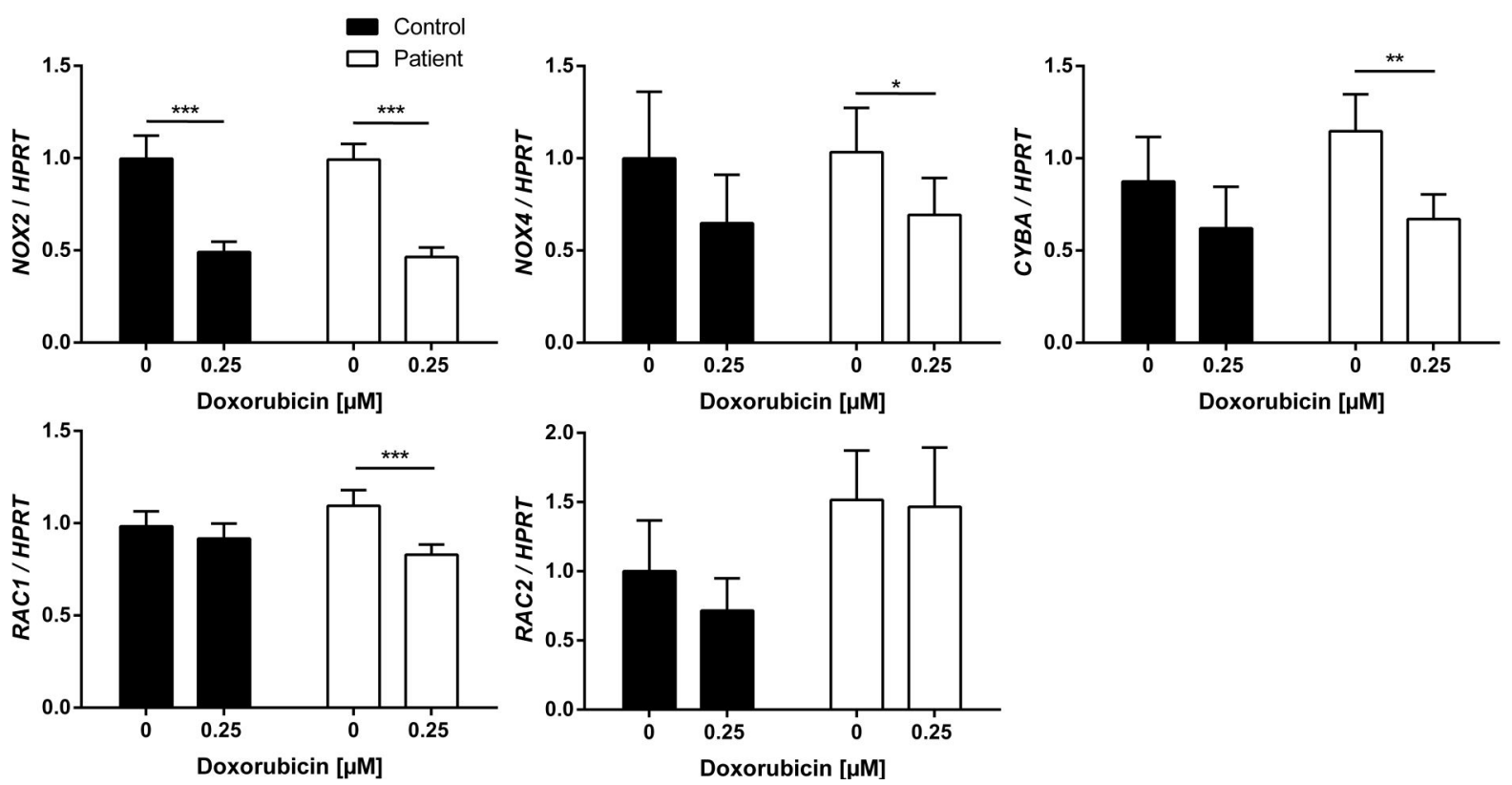

Figure 17: Expression of NADPH oxidase subunit encoding genes in iPSC-CMs on the mRNA level. Expression of NOX2, NOX4, CYBA, RAC1 and RAC2. Sample number: 11 Ctrl-iPSC-CM differentiations, 18 ACT-iPSC-CM differentiations. Mean + SEM. ${ }^{*} \mathrm{p}<0.05,{ }^{* *} \mathrm{p}<0.01, * * * \mathrm{p}<0.001$.

Using SDS-PAGE and western blotting, the expression of NOX2, NOX4, p22phox (encoded by CYBA), RAC1, RAC2 and p40phox (encoded by NCF4) was investigated on the protein level (Figure 18). Experiments were performed by Dr. med. Andreas Petry from the German Heart Centre, Munich. The amount of NADPH oxidase subunits in iPSC-CMs was low and in some cases at the detection limit. Hence, no reproducible results could be obtained for p22phox and RAC2. Furthermore, a high heterogeneity between cell lines of the same donor was observed (Figure $18 \mathrm{~B}$ ). At basal conditions, ACT-iPSC-CMs contained significantly more p40phox compared to Ctrl-iPSC-CMs, whereas a comparable amount of NOX2, NOX4 and RAC1 was detected (Figure $18 \mathrm{~A}$, untreated). After treatment with $0.25 \mu \mathrm{M}$ DOX for 24 hours, a significant increase in the amount of p40phox was observed in Ctrl-iPSC-CMs, whereas RAC1 was significantly upregulated upon $1 \mu \mathrm{M}$ DOX application in ACT-iPSC-CMs. The proteins were also analyzed 48 hours after finishing DOX application for 24 hours. The amount of NOX2, NOX4, p40phox and RAC1 remained unchanged in ACT-iPSC-CMs. In the control group on the other hand, a significant downregulation of p40phox to about $50 \%$ concentration was observed. 
A
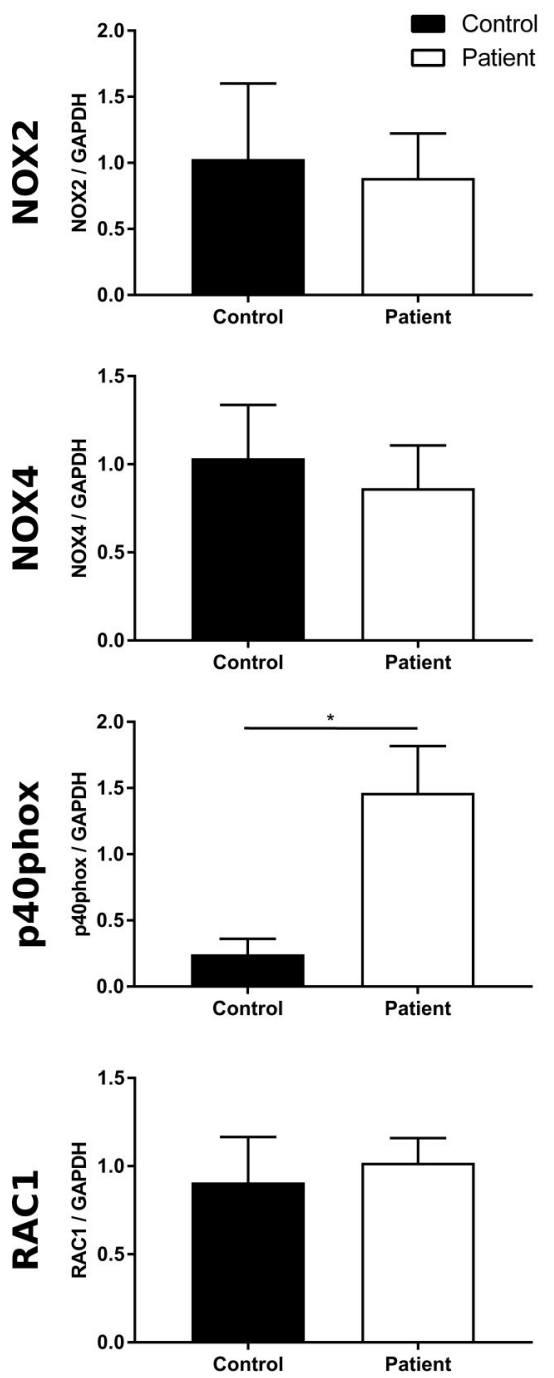

B

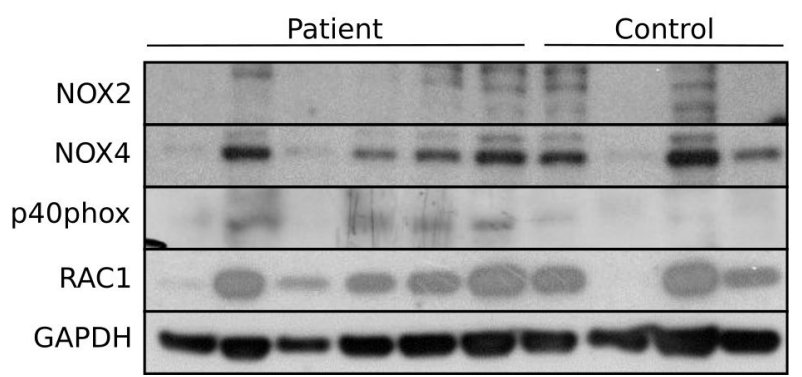

Oh after DOX
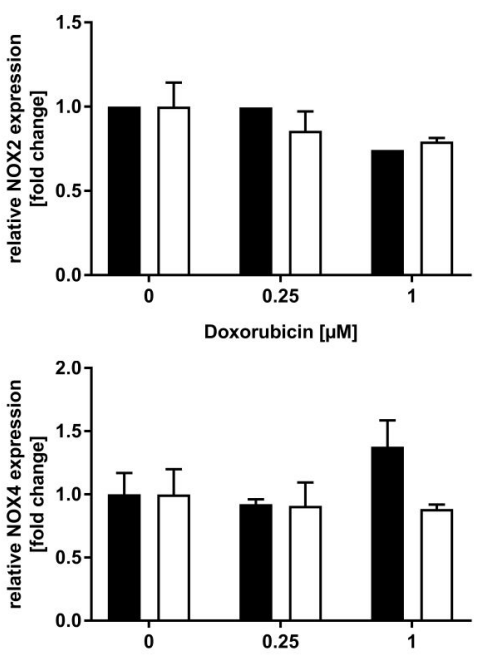

Doxorubicin [ $\mu \mathrm{M}]$

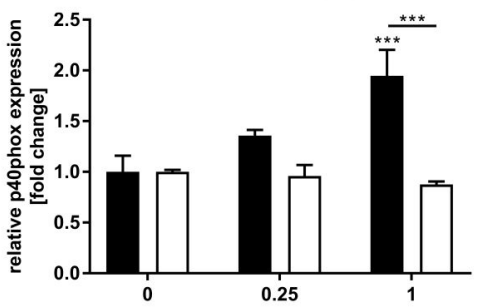

Doxorubicin [ $\mu \mathrm{M}]$

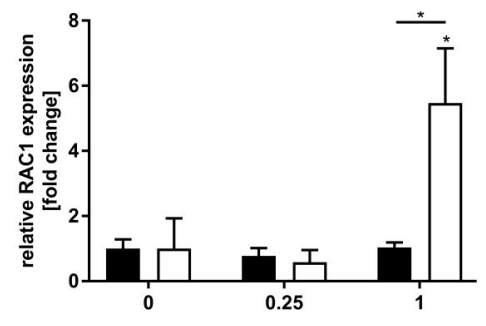

Doxorubicin $[\mu \mathrm{M}]$ 48h after DOX
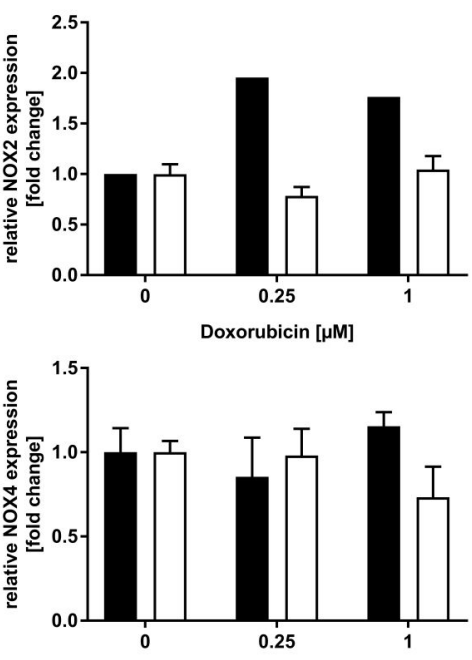

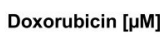

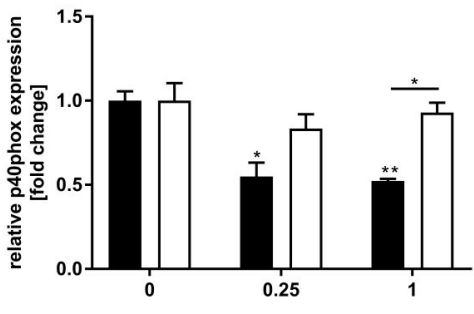

Doxorubicin $[\mu \mathrm{M}]$

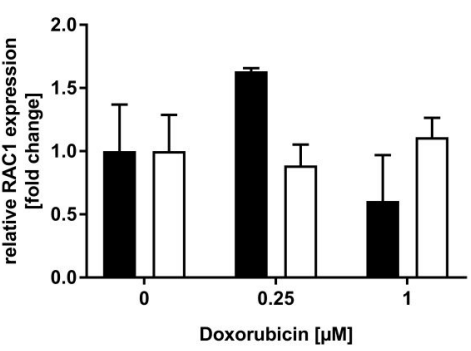

Figure 18: Western blot analyses of NADPH oxidase subunits in iPSC-CMs. A: Expression of NADPH oxidase subunits at basal conditions and $0 \mathrm{~h}$ or $48 \mathrm{~h}$ after single DOX application for $24 \mathrm{~h}$. Sample number without treatment: 4 Ctrl-iPSC-CM differentiations, 6 ACT-iPSC-CM differentiations. Sample number with DOX: 2 Ctrl-iPSC-CM differentiations (expect for NOX2. Sample number: 1), 3 ACT-iPSCCM differentiations. Mean + SEM. ${ }^{*} p<0.05, * * p<0.01, * * * p<0.001$ B: Western blot results used for quantification. Left: Untreated. Right: DOX treated. 


\subsubsection{ROS generation}

\subsubsection{Amplex Red Hydrogen Peroxide Assay}

The generation of ROS and the resulting subcellular damage in CMs is discussed as a key mechanism of ACT. Furthermore, the potentially ACT predisposing SNPs are part of genes that encode for subunits of the NADPH oxidases, which are ROS producing enzyme complexes. For these reasons, the analysis of ROS in Ctrl- and ACT-iPSC-CMs was a main aspect of this work.

The Amplex Red Hydrogen Peroxide Assay was used to assess the amount of $\mathrm{H}_{2} \mathrm{O}_{2}$ produced by iPSC-CMs at basal conditions and in response to DOX treatment (Figure 19). Although not significant, $\mathrm{H}_{2} \mathrm{O}_{2}$ production was higher in ACT-iPSC-CMs compared to Ctrl-iPSC-CMs without DOX treatment (Figure 19 A). Treatment with low, clinically relevant DOX concentrations (0.1, $0.25,0.5 \mu \mathrm{M}$ DOX) induced an increase in $\mathrm{H}_{2} \mathrm{O}_{2}$ amount, which was significant for $0.25 \mu \mathrm{M}$ DOX in both groups and for $0.5 \mu \mathrm{M}$ DOX only in ACT patients. ACT-iPSC-CMs produced higher amounts of $\mathrm{H}_{2} \mathrm{O}_{2}$ with every tested DOX concentration which was significant for $0.5 \mu \mathrm{M}$ (Figure $19 \mathrm{~A}$ ). The amount of $\mathrm{H}_{2} \mathrm{O}_{2}$ was not altered by higher DOX concentrations of $0.75,1$ and $5 \mu \mathrm{M}$ in both groups (Figure $19 \mathrm{~A}$ ). Comparing the relative DOX-induced changes in $\mathrm{H}_{2} \mathrm{O}_{2}$ amount, no differences between both groups were found (Figure 19 B). 
A

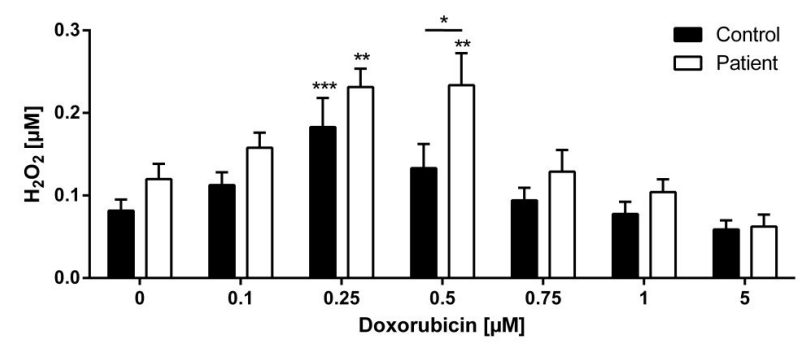

B

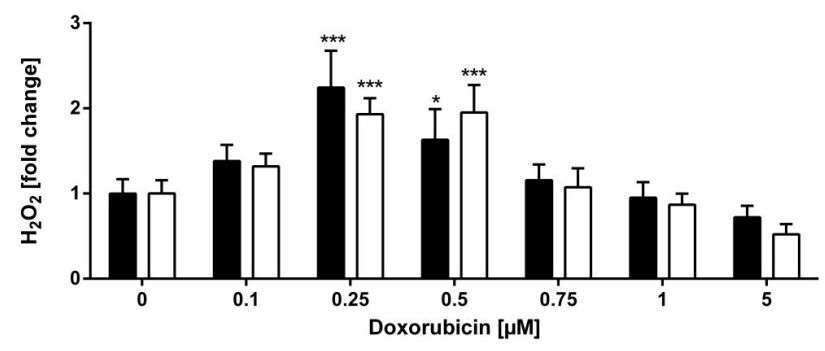

C

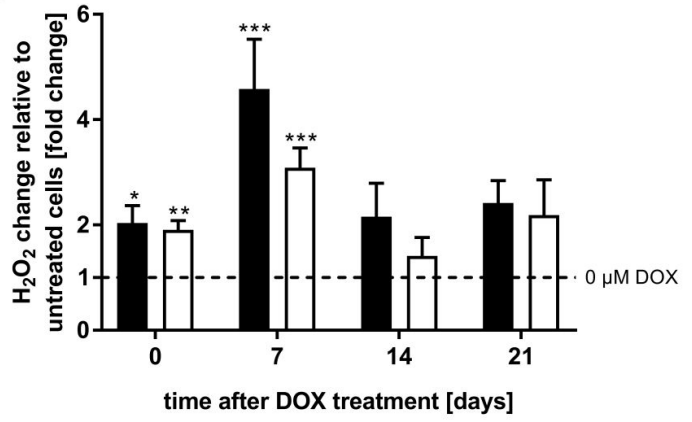

D

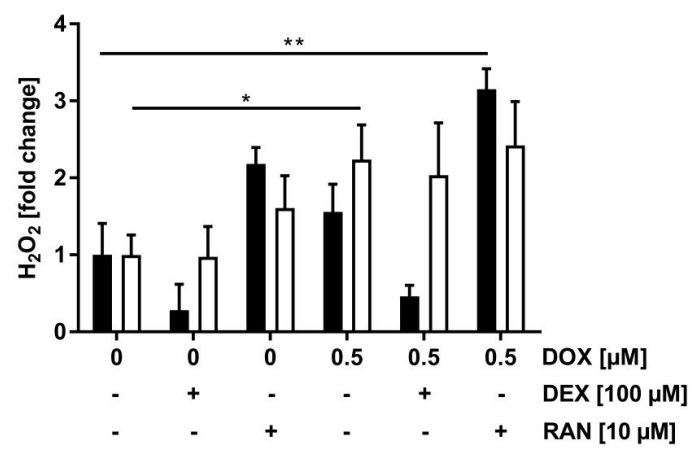

Figure 19: Analysis of extracellular $\mathrm{H}_{2} \mathrm{O}_{2}$ amount in iPSC-CMs using Amplex Red. A: $\mathrm{H}_{2} \mathrm{O}_{2}$ amount in iPSC-CMs of both groups. Sample number: 11 Ctrl-iPSC-CM differentiations, 17 ACT-iPSC-CM differentiations B: Relative DOX-induced change in $\mathrm{H}_{2} \mathrm{O}_{2}$ amount in iPSC-CMs of both groups. C: Relative change in $\mathrm{H}_{2} \mathrm{O}_{2}$ amount in iPSC-CMs of both groups $0,7,14$ and 21 days after single treatment with $0.25 \mu \mathrm{M}$ DOX for $24 \mathrm{~h}$. Sample number: 5-17 Ctrl-iPSC-CM differentiations, 13-31 ACTiPSC-CM differentiations $\mathrm{D}: \mathrm{H}_{2} \mathrm{O}_{2}$ production of iPSC-CMs of both groups after coadministration of DOX and either DEX or RAN. Sample number: 5-10 Ctrl-iPSC-CM differentiations, 7-14 ACT-iPSC-CM differentiations. Mean + SEM. ${ }^{*} p<0.05, * * p<0.01, * * * p<0.001$.

To address the question, if the observed DOX-induced changes in $\mathrm{H}_{2} \mathrm{O}_{2}$ production occurred only during or immediately after the treatment of the cells, the Amplex Red Hydrogen Peroxide Assay was applied 7, 14 and 21 days after one-time treatment for 24 hours with $0.25 \mu \mathrm{M}$ DOX (Figure $19 \mathrm{C}$ ). The relative DOX-induced changes in $\mathrm{H}_{2} \mathrm{O}_{2}$ amount was analyzed relative to untreated cells at the same time points. Interestingly, the highest changes of $\mathrm{H}_{2} \mathrm{O}_{2}$ amount was found 7 days after one-time DOX treatment in both groups. At this time point, a significant fourfold increase in the control group and a significant threefold increase in the ACT patient group was found. The $\mathrm{H}_{2} \mathrm{O}_{2}$ amount directly after treatment was significantly increased twofold in both groups. Even 14 and 21 days after one-time DOX treatment, the $\mathrm{H}_{2} \mathrm{O}_{2}$ amount was increased about twofold in both groups, which was not significant, however. These findings suggest that chronic changes were induced by single DOX application.

DEX and RAN are described as potential protectors against ACT that could be coadministered during chemotherapy. RAN was directly linked to a reduction of DOX-induced ROS (see chapter 1.1.2). For this reason, iPSC-CMs were preincubated with either $100 \mu \mathrm{M}$ DEX for 24 
hours or $10 \mu \mathrm{M}$ RAN for 48 hours. Subsequently, iPSC-CMs were cotreated with $0.5 \mu \mathrm{M}$ DOX (Figure 19 D). DEX caused a decrease of $\mathrm{H}_{2} \mathrm{O}_{2}$ amount in Ctrl-iPSC-CMs without DOX treatment, which was not significant. No such difference was observed in ACT-iPSC-CMs. The $\mathrm{H}_{2} \mathrm{O}_{2}$ amount in the control group cotreated with DOX and DEX was lower than in untreated cells, whereas DOX treatment by itself induced a twofold increase. DEX application did not alter the DOX-induced increase in $\mathrm{H}_{2} \mathrm{O}_{2}$ amount in ACT-iPSC-CMs. RAN, on the other hand, caused an increased amount of $\mathrm{H}_{2} \mathrm{O}_{2}$ in the control group without DOX. Coadministration even enhanced the DOX-induced rise of $\mathrm{H}_{2} \mathrm{O}_{2}$ significantly in Ctrl-iPSC-CMs. No RAN-induced changes were observed in the ACT-iPSC-CMs without or with DOX treatment.

\subsubsection{2 roGFP2-based biosensor analyses}

The roGFP2-based biosensors roGFP2-Orp1 and Grx1-roGFP2 were used in living iPSC-CMs to assess the level of ROS (Figure 20, 21 and 22). Since iPSC-CMs are hard to transfect via lipofection with vectors as big as the biosensors, a lentiviral approach was used to generate biosensor expressing iPSC-CMs. For this purpose, HEK-293T cells were transfected with Addgenes second generation lentiviral system including the envelope and packaging plasmids pMD2.G and psPAX2. The virus-containing cell supernatant was used for transduction of iPSC-CMs. Observed visually, a sufficient amount of iPSC-CMs usually expressed roGFP2-Orp1 or Grx1-roGFP2 72 hours after transduction start (Figure 20 A, B). Also, biosensor-expressing iPSC-CMs still showed beating activity and no differences compared to non-transduced cells were observed. However, when the cells were cultured for about one week after transduction, usually more transduced cells died than non-transduced. To avoid this, the cells were used for measurements about five days after start of transduction. When excited at 405 and $485 \mathrm{~nm}$ and measured at $515 \mathrm{~nm}$, the biosensor containing cells exhibited stronger fluorescence than non-transduced cells (Figure 20 C, D). As expected, addition of the oxidant diamide during measurement resulted in a decline of the signal at $485 \mathrm{~nm}$ excitation and a rise at $405 \mathrm{~nm}$. Addition of the reductant DTT caused the opposite effect. These observations confirm the functionality of roGFP2-Orp1 and Grx1-roGFP2 in iPSC-CMs (Figure 20 C, D). 
4 Results
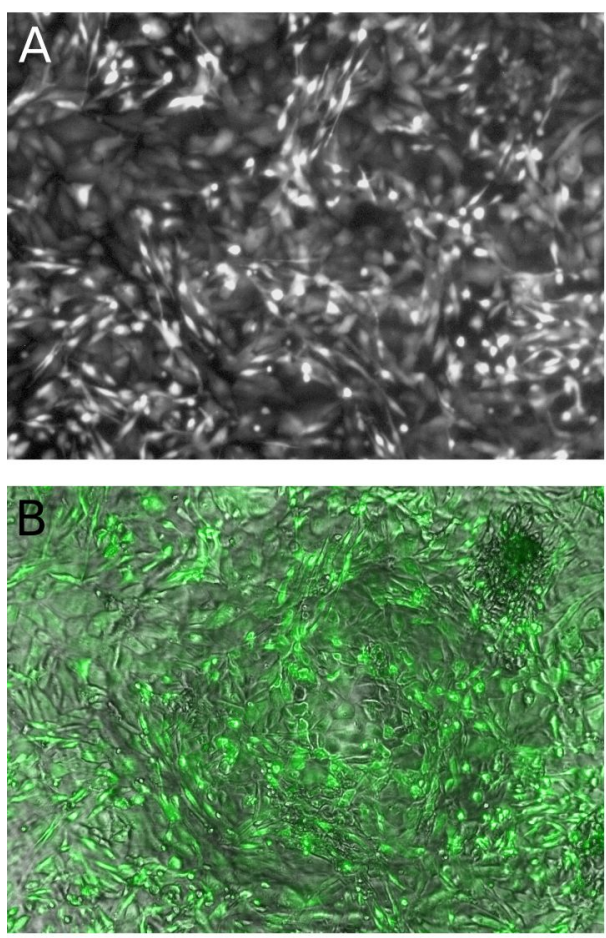

C

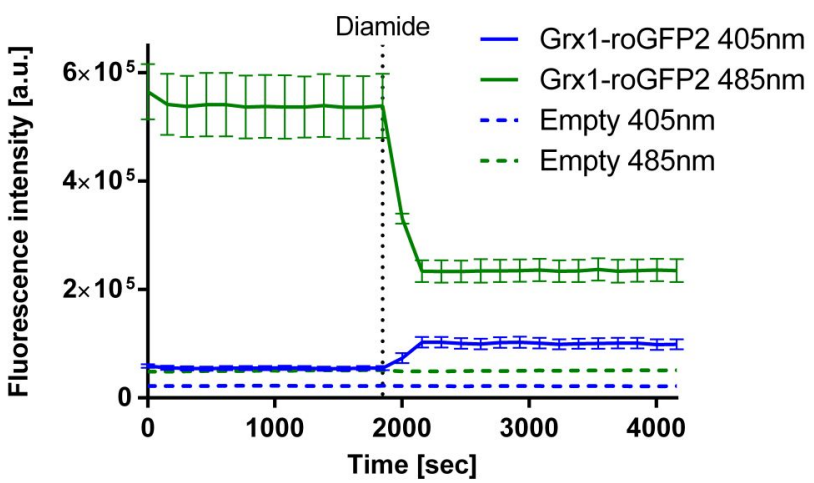

$\mathrm{D}$

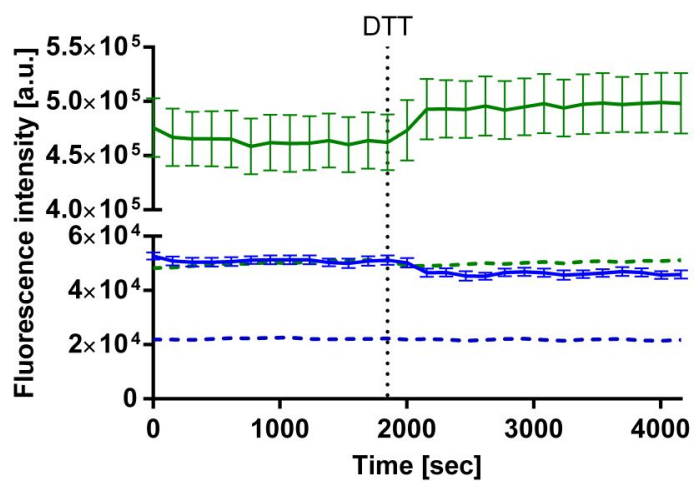

Figure 20: roGFP2-based biosensors roGFP2-Orp1 and Grx1-roGFP2 in iPSC-CMs. A, B: roGFP2-Orp1 expression in iPSC-CMs (representative for Grx1-roGFP2). C, D: Detection of Grx-roGFP2 fluorescence intensity at $510 \mathrm{~nm}$ upon excitation with 405 and $485 \mathrm{~nm}$. Oxidation (diamide) and reduction (DTT) causes respective dynamic changes in fluorescence intensity. Empty: non-transduced iPSC-CMs. Depicted measurements are representative for roGFP2-Orp1.

roGFP2-based biosensor measurements may be used for relative comparisons but do not give absolute values. To enable comparability of different iPSC-CM differentiation experiments, the degree of roGFP2 oxidation (OxD) had to be determined for every measurement. For this reason, conditions of diamide and DTT treatment had to be determined where roGFP2 would be completely oxidized $(O x D=1)$ or reduced $(O x D=0)$. The 405/485nm ratio of both roGFP2-Orp1 and Grx1-roGFP2 increased after treatment with 1, 2.5, 5 and $10 \mu \mathrm{M}$ diamide, but did not further increase with higher concentrations, indicating that a plateau was reached (Figure $21 \mathrm{~A}, \mathrm{~B}$ ). Treatment of roGFP2-Orp1-expressing iPSC-CMs with $0.05,0.5,5,50$ and $90 \mathrm{mM}$ DTT only caused a mild decrease of the $405 / 485 \mathrm{~nm}$ ratio (Figure $21 \mathrm{C}$ ). However, pretreatment of Grx1-roGFP2 expressing iPSC-CMs with $\mathrm{H}_{2} \mathrm{O}_{2}$ revealed the potential of DTT to reduce the 405/485nm ratio: Adding $10 \mathrm{mM}$ DTT to iPSCCMs pretreated with up to $20 \mu \mathrm{M} \mathrm{H}_{2} \mathrm{O}_{2}$ caused the ratio to drop to the level of untreated cells (Figure 21 D). For these reasons, the concentrations of $500 \mu \mathrm{M}$ diamide and $10 \mathrm{mM}$ DTT have been chosen to induce complete oxidation and complete reduction of roGFP2 in following experiments. Using the fluorescence intensities of fully oxidized and fully reduced conditions, OxD of roGFP2 was calculated with equation 2 (see chapter 3.2.8). 
A

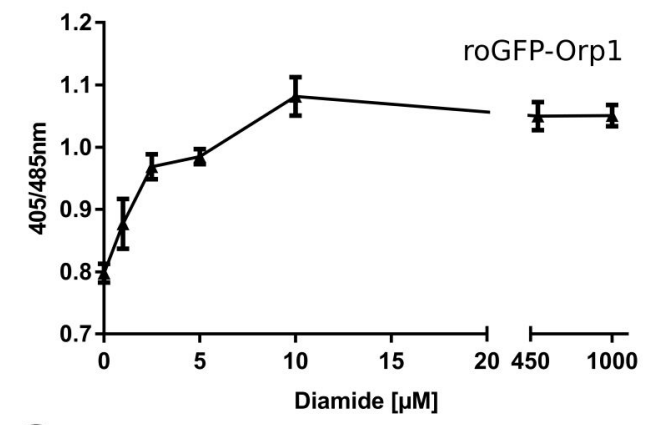

C

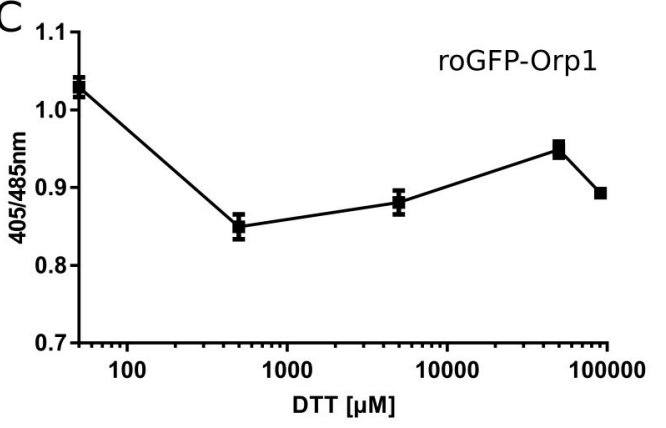

B

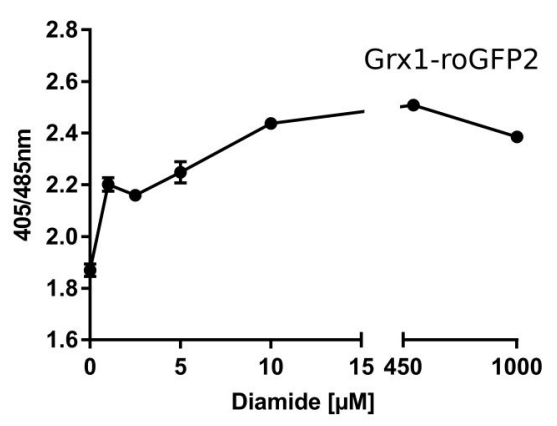

D

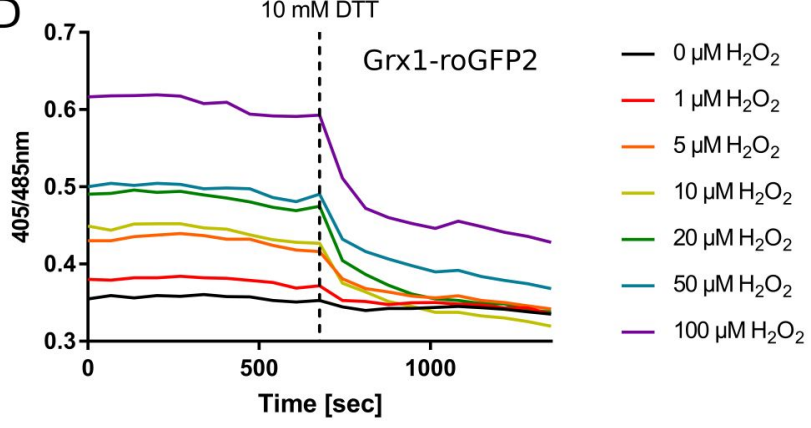

Figure 21: Determination of diamide and DTT concentrations for the induction of complete oxidation and reduction of roGFP2-Orp1 and Grx1-roGFP2 in iPSC-CMs. A: roGFP2-Orp1 oxidation in iPSC-CMs upon diamide application. B: Grx1-roGFP2 oxidation in iPSC-CMs upon diamide application. C: roGFP2-Orp1 reduction in iPSC-CMs upon DTT application. D: Grx1-roGFP2 reduction in iPSC-CMs pretreated with $\mathrm{H}_{2} \mathrm{O}_{2}$ upon DTT application.

roGFP2-Orp1 expressing Ctrl- and ACT-iPSC-CMs showed a comparable level of roGFP2 oxidation without DOX treatment (Figure 22 A). DOX treatment caused a dose-dependent increase in oxidation in both groups of up to 2.5-fold, which was significant for DOX concentrations of $0.75,1$ and $5 \mu \mathrm{M}$ (Figure $22 \mathrm{~A}, \mathrm{~B}$ ). 
A

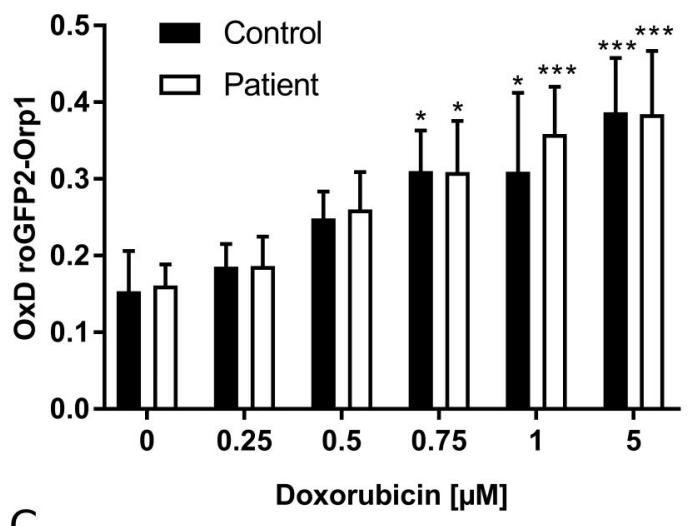

C

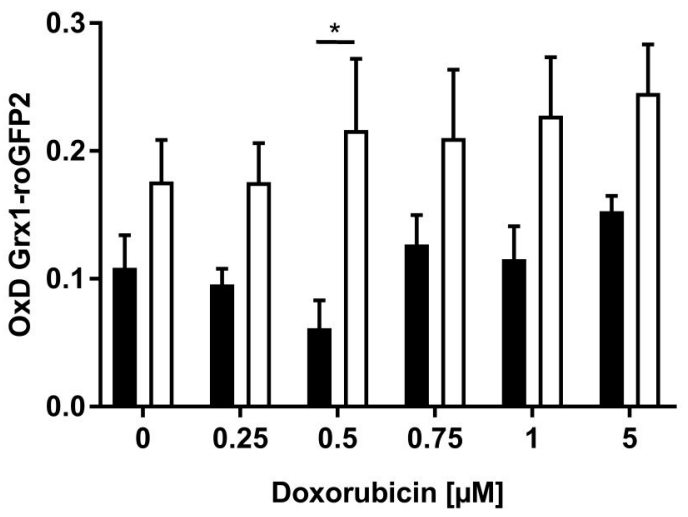

B
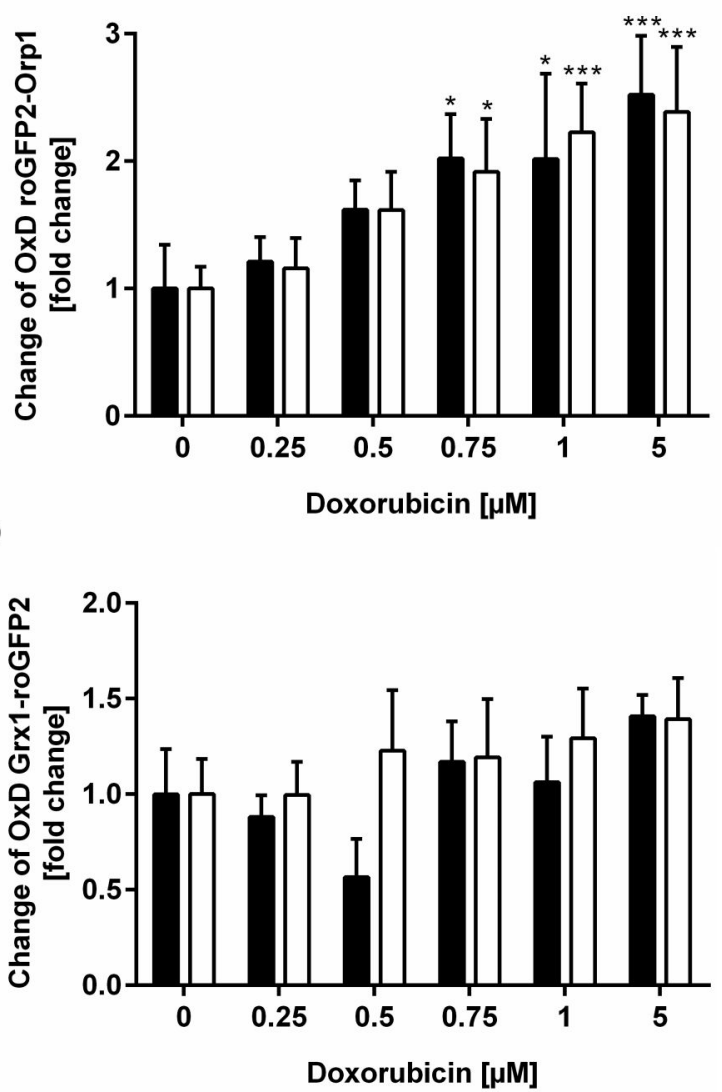

Figure 22: Analysis of ROS in iPSC-CMs using genetically encoded biosensors roGFP2-Orp1 and Grx1-roGFP2. A: $\mathrm{H}_{2} \mathrm{O}_{2}$ production in iPSC-CMs of both groups represented by roGFP2-Orp1 OxD. B: Relative DOX-induced change in roGFP2-Orp1 OxD. Sample number: 5 Ctrl-iPSC-CM differentiations, 8 ACT-iPSC-CM differentiations. C: Grx1-roGFP2 oxidation in iPSC-CMs of both groups. D: Relative DOX-induced change in Grx1-roGFP2 OxD. Sample number: 5 Ctrl-iPSC-CM differentiations, 6 ACT-iPSC-CM differentiations. Mean + SEM. ${ }^{*} \mathrm{p}<0.05, * * \mathrm{p}<0.01,{ }^{* * *} \mathrm{p}<$ 0.001 .

Analyses of Grx1-roGFP2 redox state revealed a higher oxidation in ACT-iPSC-CMs compared to the Ctrl-iPSC-CMs without DOX as well as after treatment with all tested DOX concentrations (Figure $22 \mathrm{C}$ ). Upon treatment with $0.5 \mu \mathrm{M}$ DOX, OxD of Grx1-roGFP2 was significantly higher in ACT-iPSC-CMs, compared to Ctrl-iPSC-CMs. A mild decrease of oxidation was observed in the control group after treatment with low DOX concentrations of 0.25 and $0.5 \mu \mathrm{M}$. Overall, no clear DOX dose-dependency was observed in iPSC-CMs of both groups. Relative changes induced by DOX were comparable between Ctrl- and ACT-iPSC-CMs. $5 \mu \mathrm{M}$ DOX treatment caused a 1.4-fold increase in both groups (Figure $22 \mathrm{D}$ ). $\mathrm{E}_{\mathrm{GSH}}$ was calculated and is shown in Table 4. Treatment with $5 \mu \mathrm{M}$ DOX caused an oxidation of about $6 \mathrm{mV}$. Taken together, oxidation of Grx1-roGFP2 was greater in iPSC-CMs of ACT patients compared to controls. 
Table 4: $E_{G S H}$ in iPSC-CMs. $E_{G S H}$ is depicted as mean \pm SEM. Sample number: 5 Ctrl-iPSC-CM differentiations, 6 ACT-iPSC-CM differentiations.

\begin{tabular}{|c|c|c|c|c|c|c|}
\hline & \multicolumn{6}{|c|}{$\operatorname{DOX}[\mu \mathrm{M}]$} \\
\hline & $\mathbf{0}$ & 0.25 & 0.5 & 0.75 & 1 & 5 \\
\hline Control & $\begin{array}{r}-301.46 \pm \\
3.83 \mathrm{mV}\end{array}$ & $\begin{array}{r}-300.79 \pm \\
2.72 \mathrm{mV}\end{array}$ & $\begin{array}{r}-297.95 \pm \\
3.74 \mathrm{mV}\end{array}$ & $\begin{array}{r}-299.02 \pm \\
4.33 \mathrm{mV}\end{array}$ & $\begin{array}{r}-296.60 \pm \\
3.03 \mathrm{mV}\end{array}$ & $\begin{array}{r}-295.16 \pm \\
2.74 \mathrm{mV}\end{array}$ \\
\hline Patient & $\begin{array}{r}-308.15 \pm \\
2.90 \mathrm{mV}\end{array}$ & $\begin{array}{r}-309.38 \pm \\
2.12 \mathrm{mV}\end{array}$ & $\begin{array}{r}-321.06 \pm \\
7.48 \mathrm{mV}\end{array}$ & $\begin{array}{r}-305.62 \pm \\
2.71 \mathrm{mV}\end{array}$ & $\begin{array}{r}-307.55 \pm \\
3.43 \mathrm{mV}\end{array}$ & $\begin{array}{r}-302.15 \pm \\
1.13 \mathrm{mV}\end{array}$ \\
\hline
\end{tabular}

\subsubsection{Calcium Handling}

$\mathrm{Ca}^{2+}$ belongs to the most important second messengers in $\mathrm{CMs}$ since it is the basis for contraction and relaxation, determines electrophysiological properties of the cell and is involved in gene transcription (see chapter 1.1.1.3). Cytosolic $\mathrm{Ca}^{2+}$ transients were visualized by loading iPSC-CMs with the $\mathrm{Ca}^{2+}$ sensitive dye Fluo- 4 and the use of confocal laser scanning microscopy. Thereby, the $\mathrm{Ca}^{2+}$ transient rise time, the relative amplitude and the decay time could be assessed (Figure 23). Isoprenaline, which activates $\beta$-adrenergic signaling, was used to additionally stimulate the iPSC-CMs.

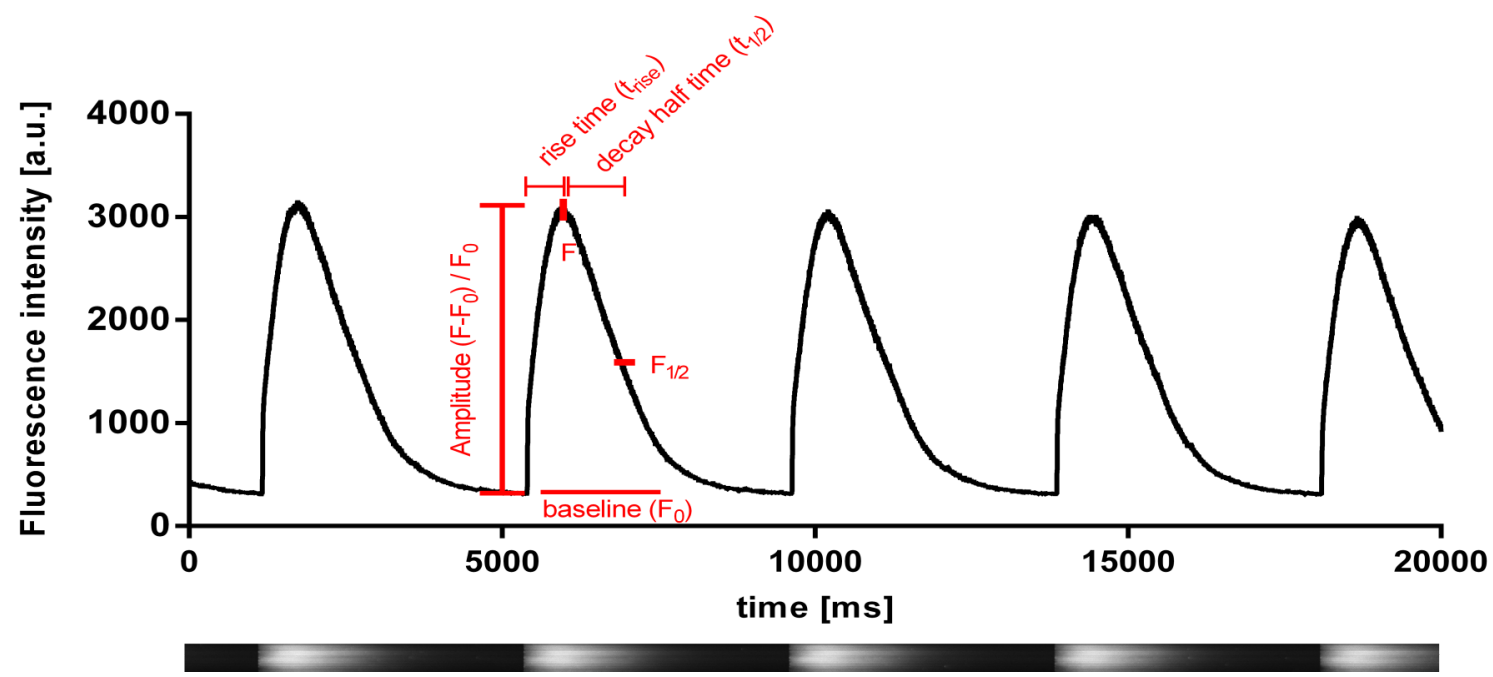

Figure 23: $\mathrm{Ca}^{2+}$ transient visualization. $\mathrm{Ca}^{2+}$ transients were analyzed using Fluo-4 live cell staining and confocal laser scanning microscopy. A suitable region in the cytoplasm of iPSC-CMs was measured for $20 \mathrm{sec}$ with the line scanning mode and relative fluorescence intensities were plotted. The $\mathrm{Ca}^{2+}$ transient rise time, amplitude and decay time were analyzed.

The $\mathrm{Ca}^{2+}$ transient rise time was comparable at basal conditions between Ctrl- and ACT-iPSCCMs (Figure $24 \mathrm{~A}$ ). It was reduced by $100 \mathrm{nM}$ isoprenaline in Ctrl- and ACT-iPSC-CMs. Treatment with $0.25 \mu \mathrm{M}$ DOX caused a significant decrease in both groups, whereas no changes were observed after $5 \mu \mathrm{M}$ DOX treatment. After $0.25 \mu \mathrm{M}$ DOX treatment, Ctrl-iPSC- 
CMs depicted a significantly faster rise time than ACT-iPSC-CMs (Figure 24 A).The $\mathrm{Ca}^{2+}$ transient amplitude of untreated iPSC-CMs from both groups was comparable and no changes were induced by isoprenaline (Figure $24 \mathrm{~B}$ ). $0.25 \mu \mathrm{M}$ DOX treatment, however, induced a significant amplitude increase in both groups, which was significantly higher in Ctrl-iPSC-CMs. Interestingly, the amplitude was significantly decreased by treatment with 5 $\mu \mathrm{M}$ DOX in both groups (Figure $24 \mathrm{~B}$ ).

Ctrl-iPSC-CMs depicted a significantly higher $\mathrm{Ca}^{2+}$ transient decay time at basal conditions than ACT-iPSC-CMs. Furthermore, isoprenaline induced a significant decrease in Ctrl-iPSCCMs but not in ACT-iPSC-CMs. A significant decrease of decay time was observed in both groups after treatment with 0.25 and $5 \mu \mathrm{M}$ DOX, which was not altered by further isoprenaline addition (Figure $24 \mathrm{C}$ ). The absolute decay time was comparable between both groups after DOX treatment (Figure $24 \mathrm{C}$ ). The relative fold change was significantly greater in the control group (Figure $24 \mathrm{D}$ ).

A

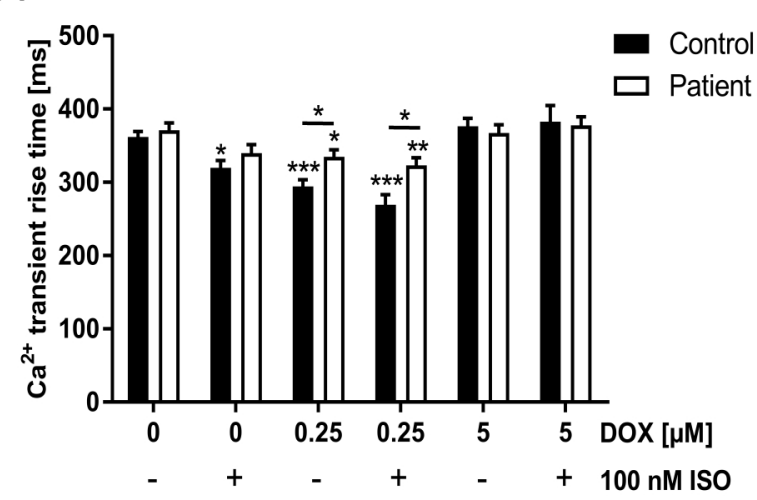

C

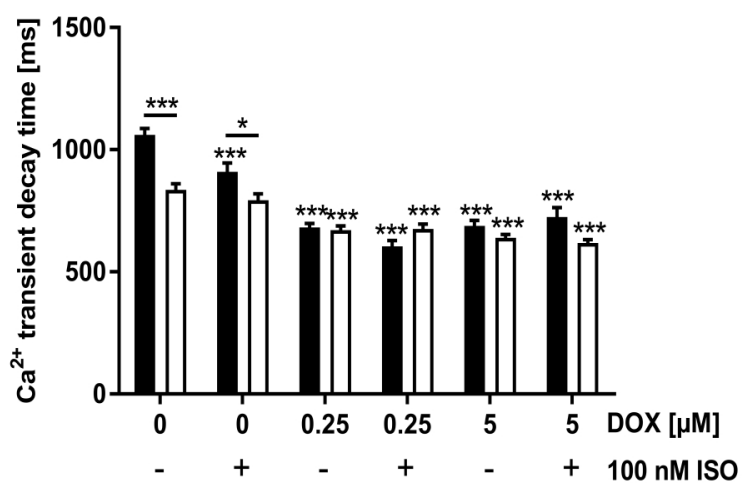

B
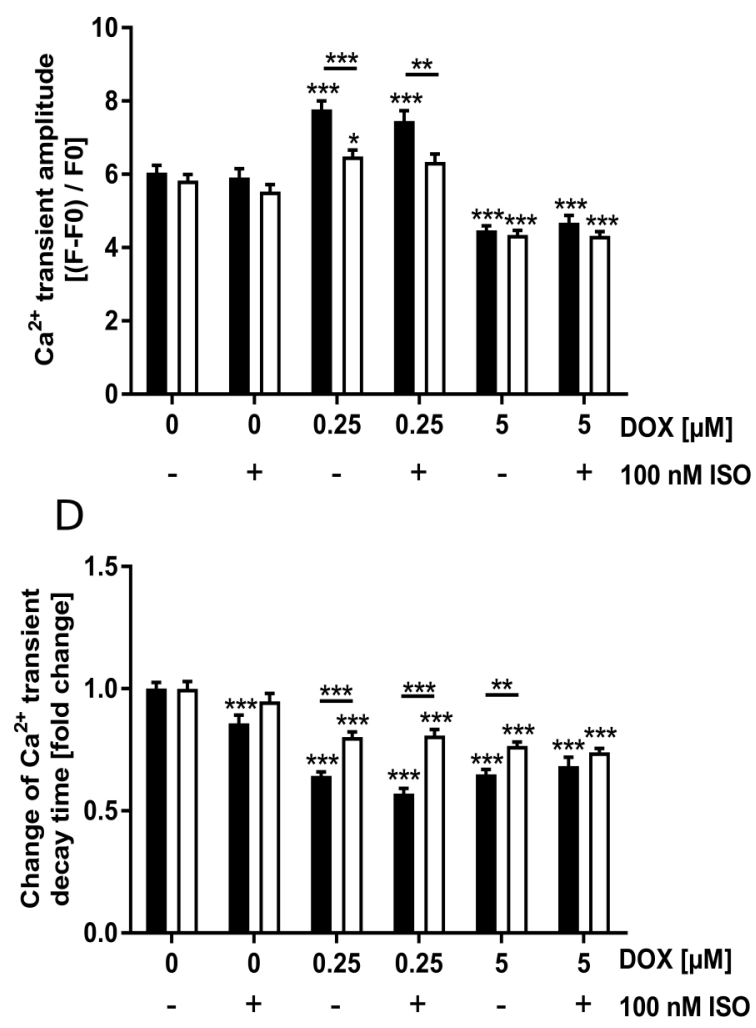

Figure 24: $\mathrm{Ca}^{2+}$ transient rise time, amplitude and decay time. $\mathrm{A}: \mathrm{Ca}^{2+}$ transient rise time in iPSC-CMs from both groups. B: $\mathrm{Ca}^{2+}$ transient amplitude in iPSC-CMs from both groups. $\mathrm{C} \mathrm{Ca}^{2+}$ transient decay time in iPSC-CMs from both groups. D: Relative changes of $\mathrm{Ca}^{2+}$ transient decay time upon application of DOX and ISO. Sample number: 200-61 Ctrl-iPSC-CMs from 9 differentiations, 197-119 ACT-iPSCCMs from 10 differentiations. Mean + SEM. $* p<0.05,{ }^{* *} p<0.01,{ }^{* * *} p<0.001$.

To assess, whether the observed changes of $\mathrm{Ca}^{2+}$ transient parameters originate from changes in gene expression, qRT-PCR and western blot analyses were performed (Figure 25, 
26 and 27). $\mathrm{Ca}^{2+}$ transient rise time and amplitude are directly influenced by RYR2 which releases $\mathrm{Ca}^{2+}$ from the SR into the cytoplasm during systole. RYR2 phosphorylation at S2808 by PKA and at S2814 by CamKII causes its activation. The amount of RYR2 mRNA was significantly greater in the Ctrl-iPSC-CMs than in ACT-iPSC-CMs and CamKII was expressed slightly more in the control group. Treatment with $0.25 \mu \mathrm{M}$ DOX resulted in significantly decreased expression of RYR2 in Ctrl-iPSC-CMs and no changes in CamKII expression (Figure 25). $\mathrm{Ca}^{2+}$ transient decay time is regulated by expression of SERCA, NCX, PLN and its phosphorylation at S16 and Thr17. No significant differences in expression of SERCA, PLN or $N C X$ was found on the mRNA level between both groups. DOX treatment caused a downregulation of SERCA and NCX mRNA in iPSC-CMs of both groups but not of PLN (Figure 25).
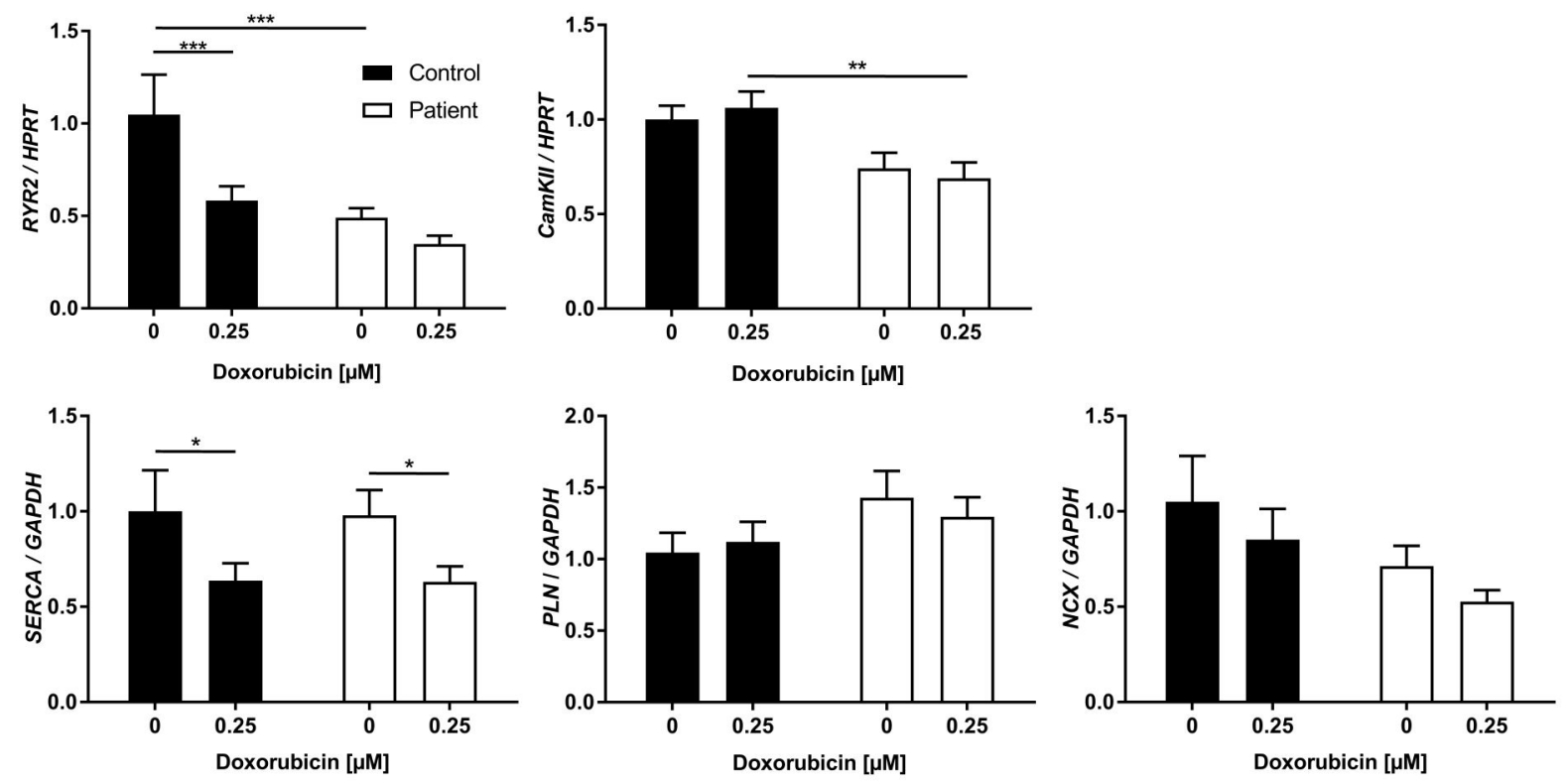

Figure 25: Expression of genes encoding for proteins involved in calcium signaling. Expression of RYR2, CamKII, SERCA, PLN and NCX. Sample number: 11 Ctrl-iPSC-CM differentiations, 18 ACT-iPSC$\mathrm{CM}$ differentiations. Mean + SEM. ${ }^{*} p<0.05, * * p<0.01, * * * p<0.001$.

At basal conditions, no significant differences were found between Ctrl- and ACT-iPSC-CMs on the protein level of RYR2 and CamKII or in the phosphorylation of RYR2 at S2808 or S2814 (Figure 26). The amount of RYR2 in Ctrl-iPSC-CMs was increased after treatment with 0.25 , 0.5 and $1 \mu \mathrm{M}$ DOX, but without significance. No changes were observed in ACT-iPSC-CMs (Figure 26 B). No significant changes were observed in phosphorylation of RYR2 at S2808 in both groups (Figure 26 D). Phosphorylation of RYR2 at S2814 increased in ACT-iPSC-CMs DOX-dependently with a significant increase at $5 \mu \mathrm{M}$ DOX compared to basal conditions and Ctrl-iPSC-CMs. It was not changed in Ctrl-iPSC-CMs (Figure 26 F). No DOX-induced changes were found in the amount of CamKII (Figure $26 \mathrm{H}$ ). 
A

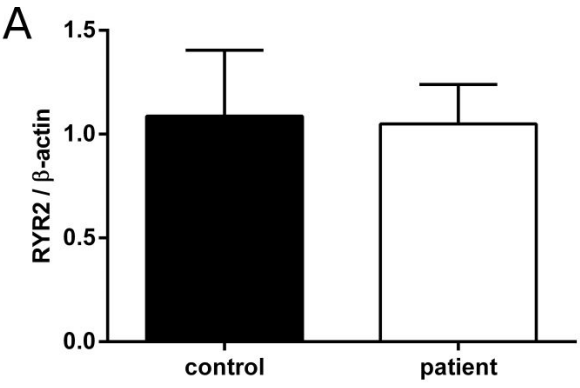

$\mathrm{C}$

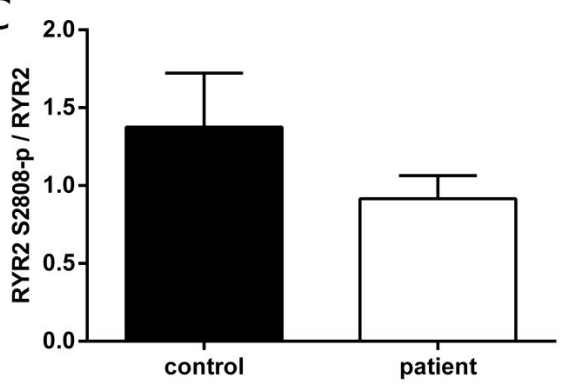

$\mathrm{E}$

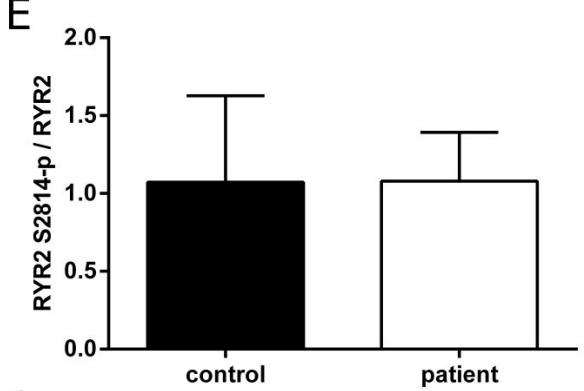

G

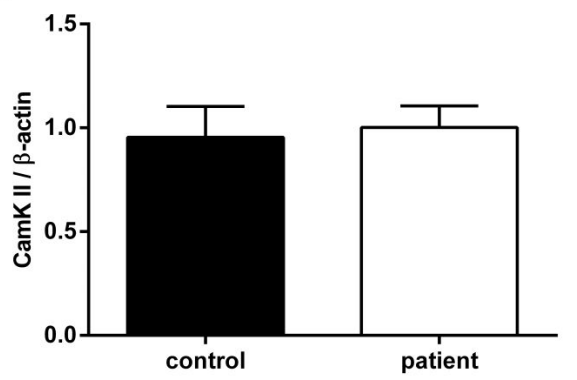

I

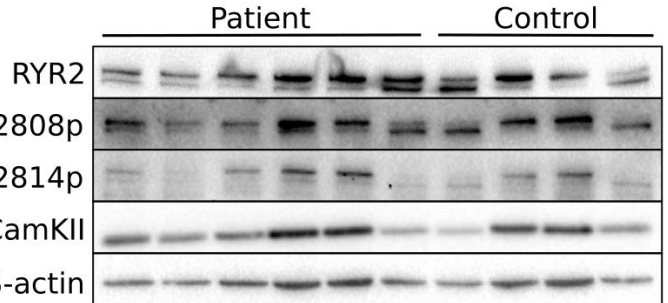

B

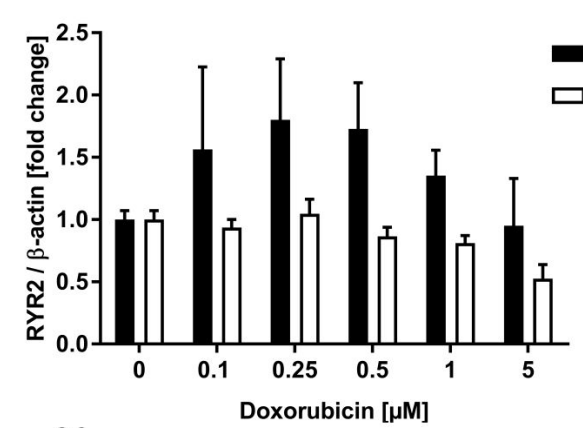

D
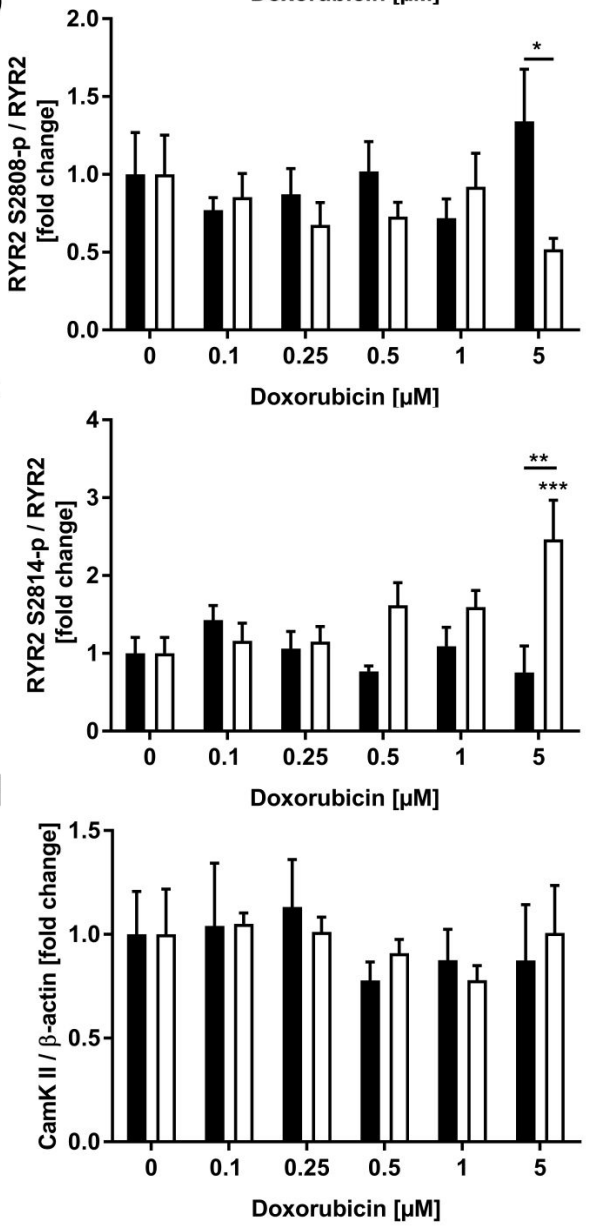

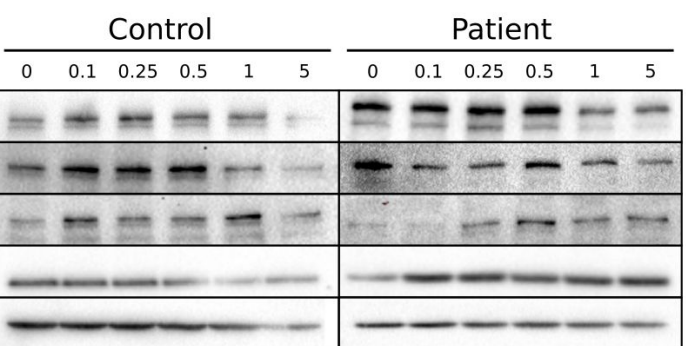

Figure 26: Western blot analyses of proteins involved in $\mathrm{Ca}^{2+}$ transient rise time and amplitude. $\mathrm{A}$, C, E, G: RYR2, RYR2-S2808p, RYR2-S2814p and CamKII in iPSC-CMs of both groups at basal conditions. B, D, F, H: DOX-induced relative changes in the amount of RYR2, RYR2-S2808p, RYR2-S2814p and CamKII in iPSC-CMs of both groups. Sample number: 4 Ctrl-iPSC-CM differentiations, 6 ACT-iPSC-CM differentiations. Mean + SEM. $* p<0.05, * * p<0.01, * * * p<0.001$. I: Representative western blot results used for quantification. Left: Untreated. Right: DOX treated. 
No significant differences were found between the control and ACT patient group in the amount of SERCA, NCX and PLN on the protein level in untreated iPSC-CMs (Figure $27 \mathrm{~A}, \mathrm{~B}$, E). At basal conditions ACT-iPSC-CMs were phosphorylated about twice as much at S16 and Thr17 of PLN, which was not significant (Figure 27 C, D). Strikingly, DOX treatment induced an increase of SERCA in Ctrl-iPSC-CMs but not in ACT-iPSC-CMs. After treatment with 0.5, 1 and $5 \mu \mathrm{M}$ DOX, its amount was significantly greater in Ctrl-iPSC-CMs compared to ACT-iPSC-CMs (Figure $27 \mathrm{~F}$ ). No DOX-induced changes were found in the amount of NCX or PLN (Figure 27 $\mathrm{G}, \mathrm{J})$. However, phosphorylation of PLN-S16 increased significantly in a dose-dependent manner in both groups (Figure $27 \mathrm{H}$ ). Furthermore, a decreased amount of phosphorylation was observed in the Ctrl-iPSC-CMs after DOX treatment at Thr17 of PLN. In ACT-iPSC-CMs, on the other hand, phosphorylation of PLN Thr17 was increased upon application of higher DOX concentrations (Figure $27 \mathrm{I}$ ). In summary, these experiments point out that Ctrl-iPSC-CMs show a DOX-dependent increase of SERCA expression, whereas ACT-iPSC-CMs have an increase in PLN-phosphorylation upon DOX treatment. 
A

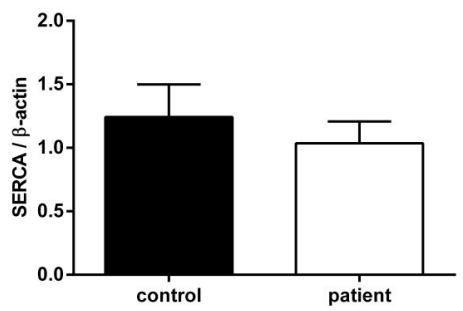

$\mathrm{D}_{2.0}$

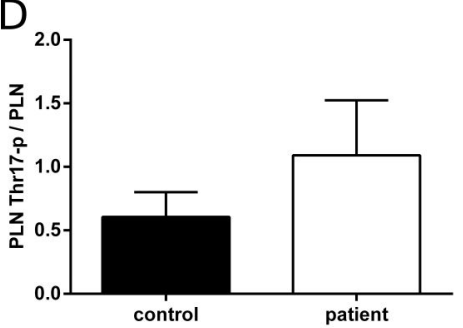

F
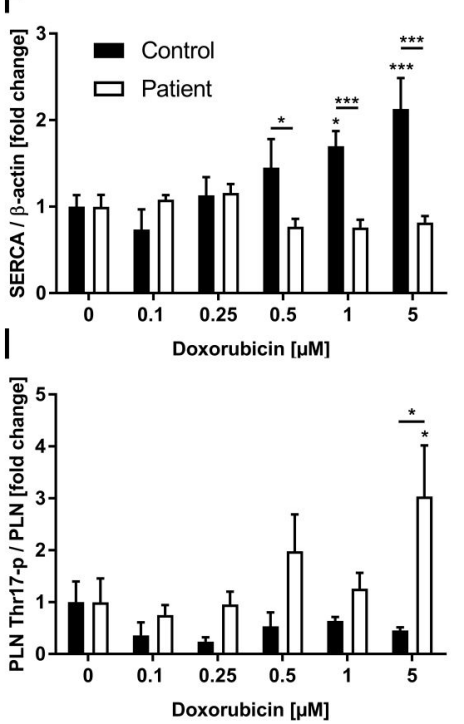

B

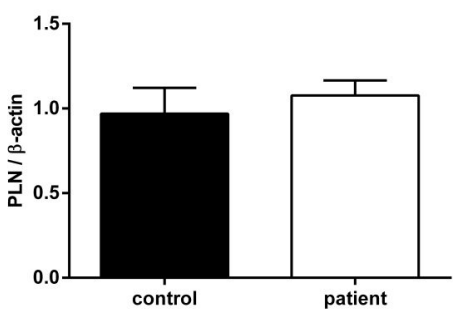

E
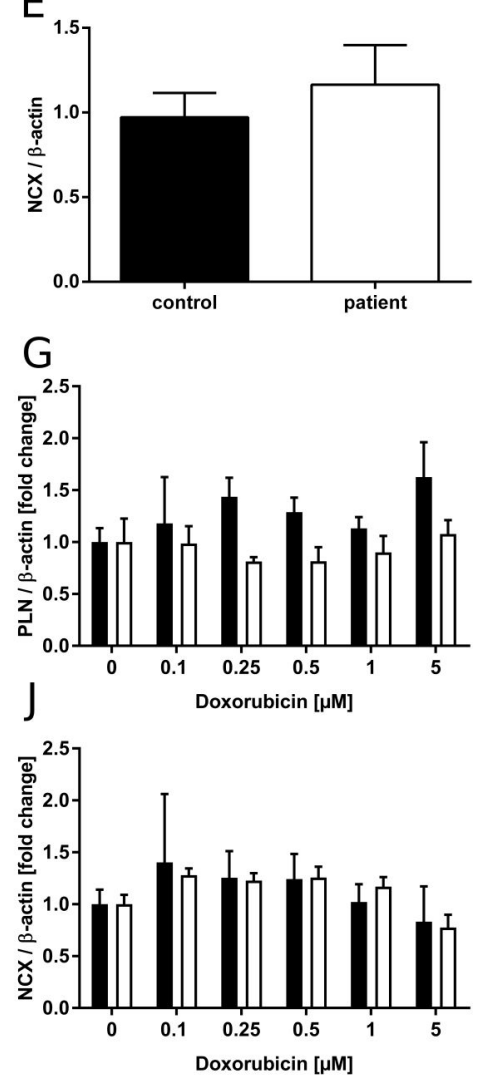

C

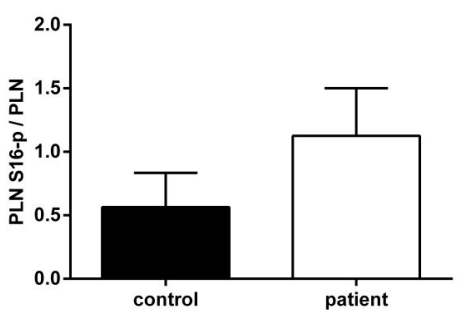

$\mathrm{H}$

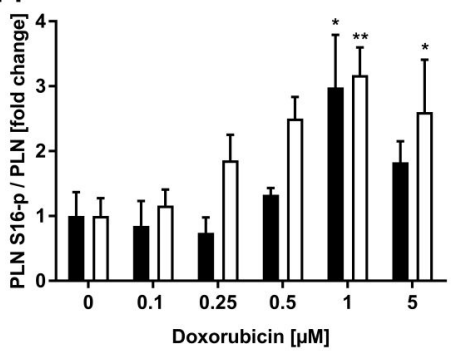

K

Control

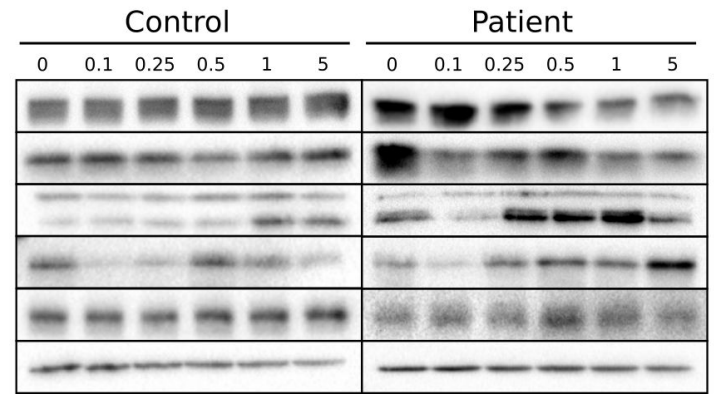

Figure 27: Western blot analyses of proteins involved in $\mathrm{Ca}^{2+}$ decay time. A-E: SERCA, PLN, PLN-S16p, PLN-Thr17p and NCX in iPSC-CMs of both groups at basal conditions. F-J: DOX-induced relative changes in the amount of SERCA, PLN, PLN-S16p, PLN-Thr17p and NCX in iPSC-CMs of both groups. Sample number: 4 Ctrl-iPSC-CM differentiations, 6 ACT-iPSC-CM differentiations. Mean + SEM. $* p<$ $0.05, * * \mathrm{p}<0.01, * * * \mathrm{p}<0.001$. K: Representative western blot results used for quantification. Left: Untreated. Right: DOX treated. 


\subsubsection{Apoptosis}

It is widely accepted that many pathomechanisms involved in ACT result in apoptosis and cell death. To analyze the rate of DOX-induced apoptosis in the iPSC-CM ACT model, the annexin $\mathrm{V}$ affinity assay was used (Figure 28). Annexin $\mathrm{V}$ binds to phosphatidylserine, which is present in the inner leaflet of the cell membrane in healthy cells. It is transported to the outer leaflet during early apoptosis. Costaining with $\mathrm{PI}$, which only enters cells with disrupted cell membranes and accumulates in the DNA, enables distinction of apoptotic and dead cells. The annexin $\mathrm{V}$ affinity assay thereby is a powerful and sensible tool to quantify apoptosis. It was applied to iPSC-CMs, which were treated with DOX for 24 or 72 hours. Although the fluorescent properties of DOX caused an overall dose-dependent signal increase in the $\mathrm{PI}$ channel, viable, apoptotic and dead cells were reliably distinguishable as three separate populations in scatterplots (Figure 28).

$0 \mu \mathrm{M}$ DOX

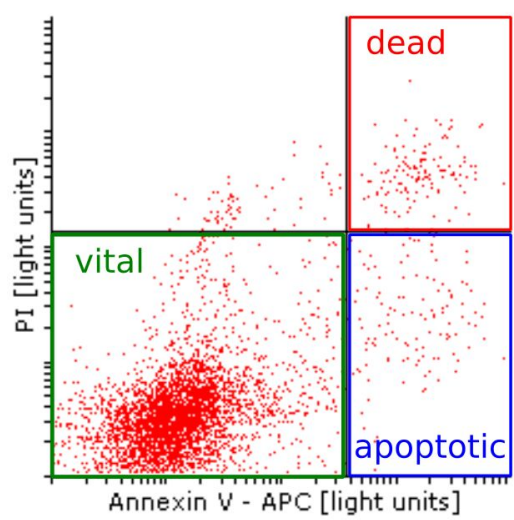

$1 \mu \mathrm{M}$ DOX

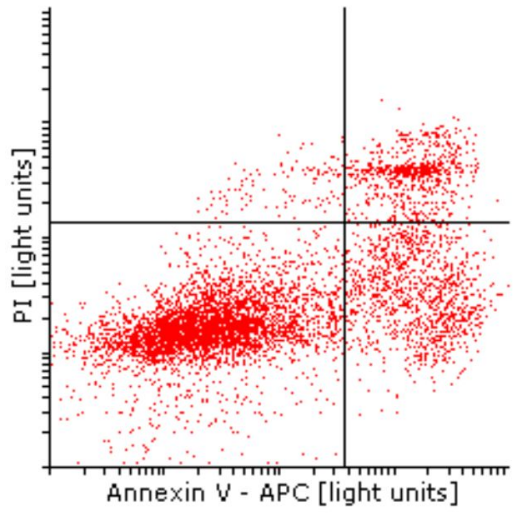

$0.25 \mu \mathrm{M}$ DOX

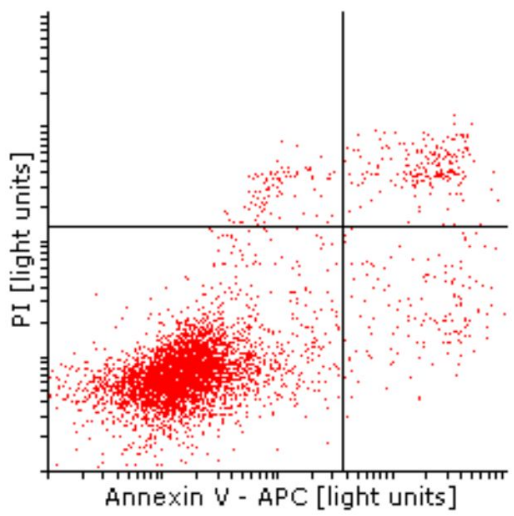

$5 \mu \mathrm{M}$ DOX

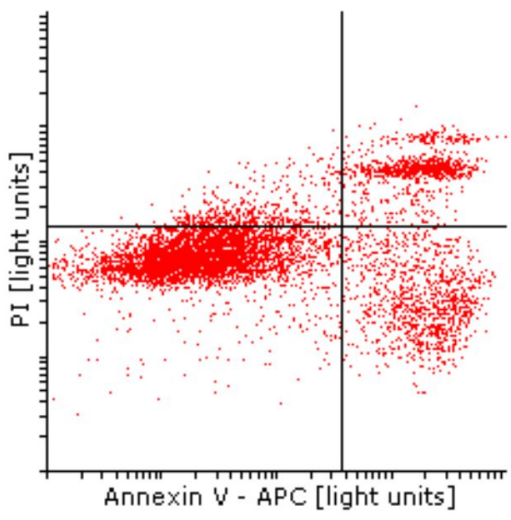

$0.5 \mu \mathrm{M}$ DOX

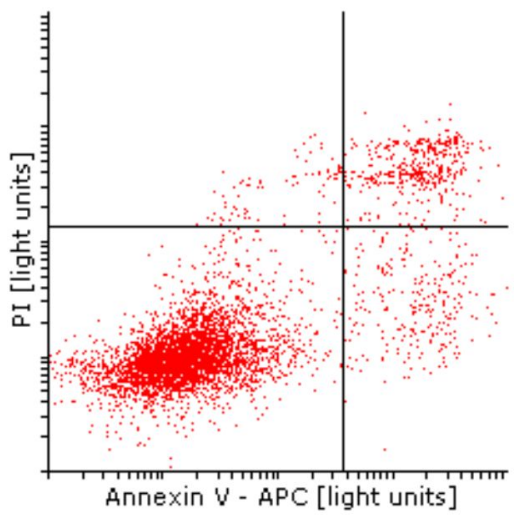

Figure 28: Flow cytometry analysis of annexin V-APC / PI staining in iPSC-CMs. Apoptotic and dead cells were detected after staining iPSC-CMs with annexin V-APC and PI with flow cytometry. Although DOX fluorescence caused an overall signal increase in the PI channel, vital (bottom left), apoptotic (bottom right) and dead cells (top right) were clearly distinguishable even at higher DOX concentrations. Images are representative for all cell lines. 
About $9 \%$ of Ctrl-iPSC-CMs were apoptotic or dead at basal conditions and treatment for 24 hours with concentrations of 0.25 or $0.5 \mu \mathrm{M}$ DOX did not cause changes. A significant amount of apoptotic or dead cells of up to $24 \%$ was found after 1 and $5 \mu \mathrm{M}$ DOX. $5 \%$ of ACTiPSC-CMs were apoptotic or dead at basal conditions. This amount was increased by 0.25 and $0.5 \mu \mathrm{M}$ DOX to about $10 \%$ and significantly by 1 and $5 \mu \mathrm{M}$ DOX up to $15 \%$ (Figure $29 \mathrm{~A}$ ). Although the absolute amount of apoptotic or dead cells was comparable between both groups after low concentrations of DOX and significantly higher in Ctrl-iPSC-CMs after high concentrations, the relative fold change was twice as high in ACT-iPSC-CMs after low DOX concentrations and comparable after high concentrations (Figure $29 \mathrm{~B}$ ).

A

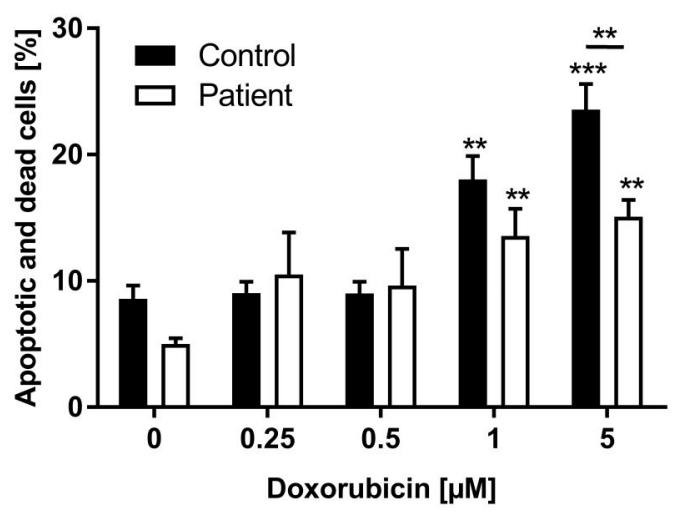

C

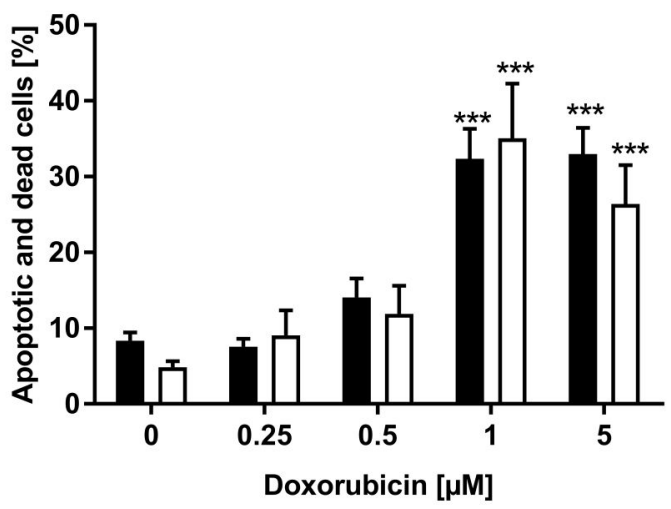

B

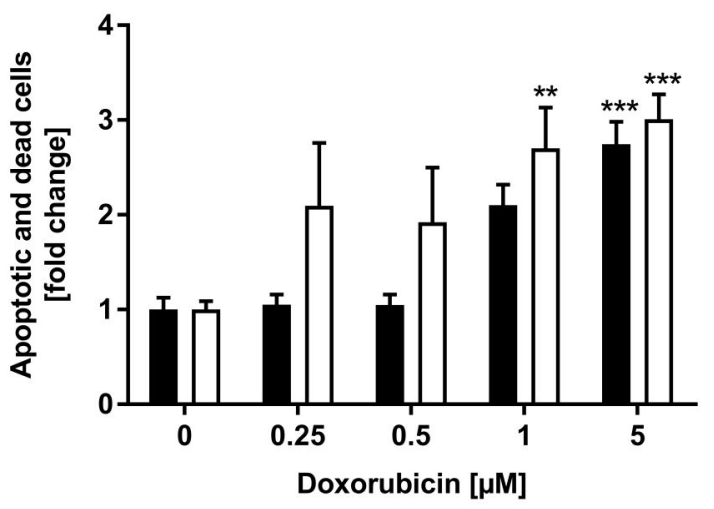

D

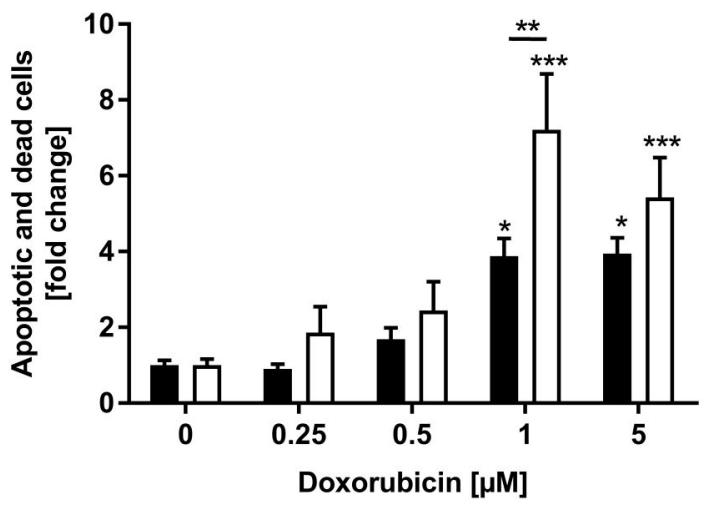

Figure 29: Analysis of apoptosis in iPSC-CMs. A: Amount of apoptotic and dead iPSC-CMs of both groups after $24 \mathrm{~h}$ DOX application. B: Relative changes in amount of apoptotic and dead iPSC-CMs of both groups after $24 \mathrm{~h}$ DOX application. C: Amount of apoptotic and dead iPSC-CMs of both groups after $72 \mathrm{~h}$ DOX application. Sample number: 13 Ctrl-iPSC-CM differentiations, 11 ACT-iPSC-CM differentiations. D: Relative changes in amount of apoptotic and dead iPSC-CMs of both groups after $72 \mathrm{~h}$ DOX application. Sample number: 10 Ctrl-iPSC-CM differentiations, 10 ACT-iPSC-CM differentiations. Mean + SEM. ${ }^{*} p<0.05,{ }^{* *} p<0.01, * * * p<0.001$.

DOX treatment for 72 hours resulted in comparable amounts of apoptotic or dead cells in both groups with around $10 \%$ after 0.25 and $0.5 \mu \mathrm{M}$ and around $30 \%$ after 1 and $5 \mu \mathrm{M}$ (Figure 29 C). However, the relative DOX-induced change was significantly higher in ACT-iPSC- 
CMs compared to Ctrl-iPSC-CMs after $1 \mu \mathrm{M}$ DOX treatment and also higher at every other tested condition (Figure $29 \mathrm{D}$ ).

\subsubsection{Sarcomeric integrity}

The development of force and overall functionality of $\mathrm{CMs}$ relies on the sarcomeric cytoskeleton, which consists of a highly organized group of proteins that form sarcomeres. The general integrity of the sarcomeric cytoskeleton depends on gene expression of respective proteins, such as $\alpha$-actinin, cTNT, $\alpha$ - and $\beta-M H C$ as well as their correct assembly into sarcomeres that are repeating with high regularity. The gene expression of sarcomeric proteins and their correct assembly are described to be disturbed by DOX, thereby contributing to decreased ejection fraction and ultimately ACT (see chapter 1.1.1.4). In the iPSC-CM ACT model, the sarcomeric regularity was investigated by immunofluorescence analyses of $\alpha$-actinin and subsequent fast Fourier transformation (FFT). Thereby, the frequency domain of the $\alpha$-actinin staining could be depicted. High regularity of sarcomeres would cause a wave pattern. The first wave represents the frequency in which neighboring sarcomeres alternate. The peak amplitude of the first wave in the frequency domain of $\alpha$ actinin stainings was used as parameter to assess the regularity of $\alpha$-actinin (Figure $30 \mathrm{~A}-\mathrm{F}$ ).

Ctrl-iPSC-CMs showed a similar $\alpha$-actinin regularity as ACT-iPSC-CMs at basal conditions (Figure $30 \mathrm{G}$ ). DOX treatment caused a mild dose-dependent decrease in Ctrl-iPSC-CMs, which was significant at $5 \mu \mathrm{M}$. In contrast, in ACT-iPSC-CMs the regularity of $\alpha$-actinin was already significantly disturbed after treatment with $0.25 \mu \mathrm{M}$ DOX. At 0.25 and $0.5 \mu \mathrm{M}$ DOX, ACT-iPSC-CM $\alpha$-actinin regularity was significantly lower compared to the control group. The decrease of $\alpha$-actinin regularity amounted for about $20 \%$, which was reached in the ACTiPSC-CMs at $0.25 \mu \mathrm{M}$ DOX and in Ctrl-iPSC-CMs after $5 \mu \mathrm{M}$ (Figure $30 \mathrm{H}$ ). 

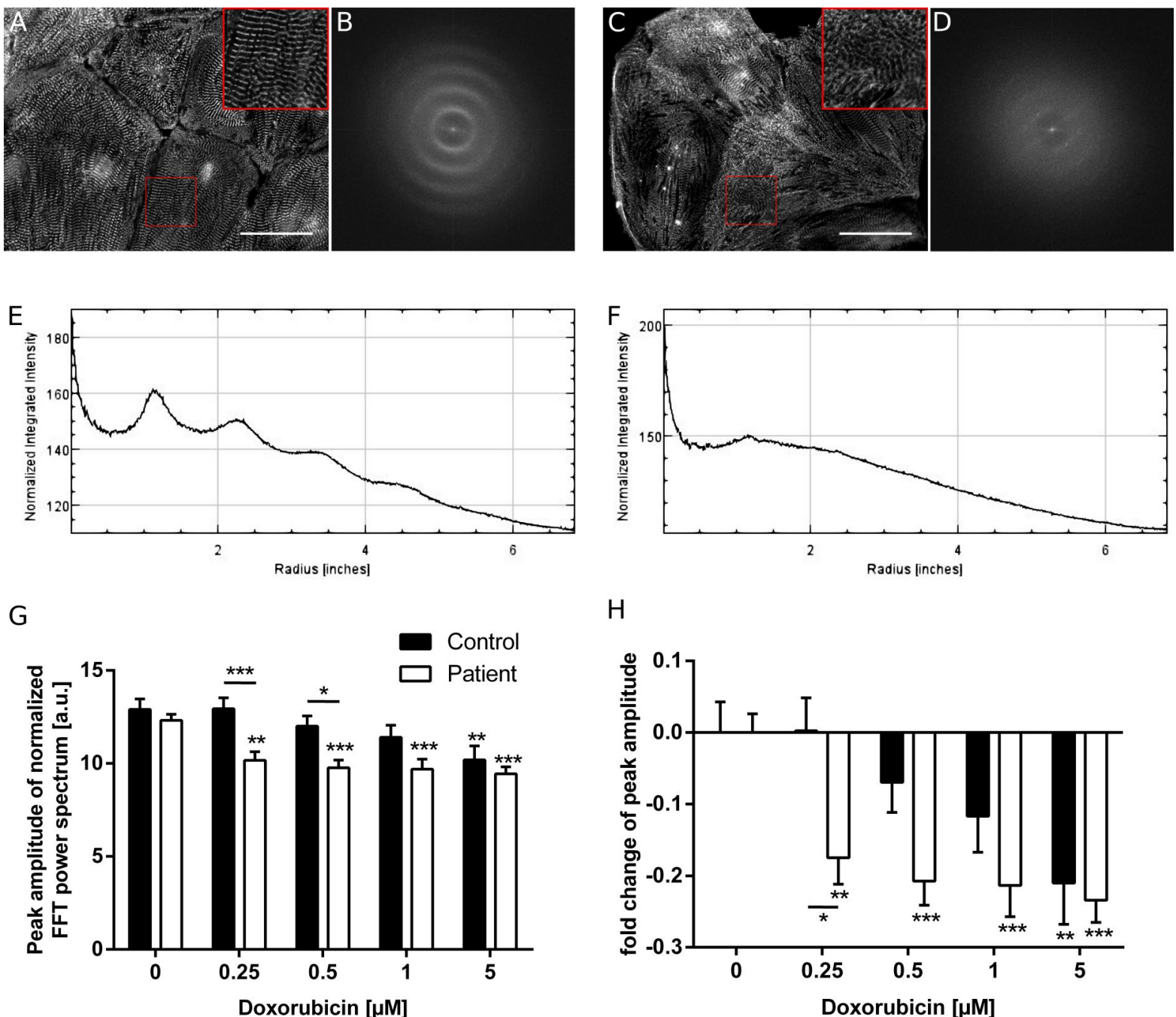

$\mathrm{H}$

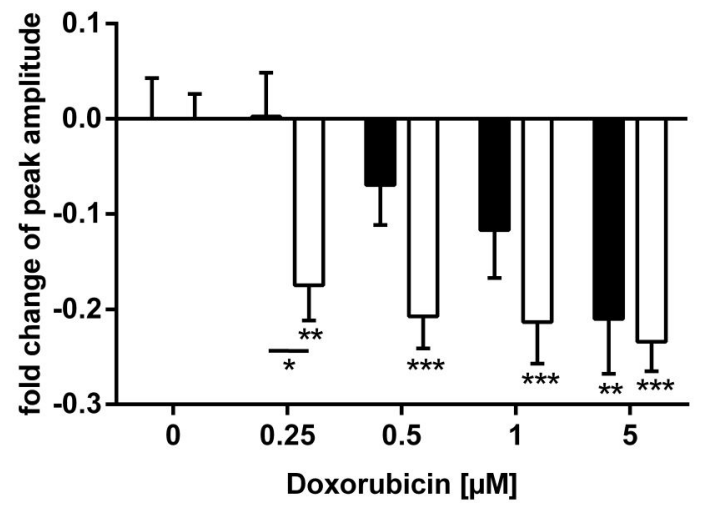

Figure 30: Integrity of sarcomeric cytoskeleton. A: $\alpha$-actinin staining in untreated iPSC-CMs. B: FFT frequency domain of untreated iPSC-CMs. C: $\alpha$-actinin staining in iPSC-CMs treated with $5 \mu \mathrm{M}$ DOX. D: FFT frequency domain of iPSC-CMs treated with $5 \mu \mathrm{M}$ DOX. E: Plot of FFT frequency domain of untreated iPSC-CMs. F: Plot of FFT frequency domain of iPSC-CMs treated with $5 \mu \mathrm{M}$ DOX. G: $\alpha-$ actinin regularity in iPSC-CMs of both groups. H: DOX-induced relative change of $\alpha$-actinin regularity in iPSC-CMs of both groups. Sample number: 62-60 Ctrl-iPSC-CMs from 4 differentiations, 91-85 ACTiPSC-CMs from 6 differentiations. Mean + SEM. ${ }^{*} p<0.05,{ }^{* *} p<0.01, * * * p<0.001$. Scale bars: $50 \mu \mathrm{m}$.

qRT-PCR analysis revealed a significant DOX-induced decrease of $\alpha$-actinin and $\beta-M H C$ expression in Ctrl-iPSC-CMs and of $\alpha$-actinin, $\alpha-M H C, \beta-M H C$ and $C T N T$ in ACT-iPSC-CMs (Figure 31 A). Furthermore, no significant dose-dependent DOX-induced changes or differences between both groups were found in the amount of $\alpha$-actinin observed by western blot analysis (Figure $31 \mathrm{~B}, \mathrm{C}$ ). 

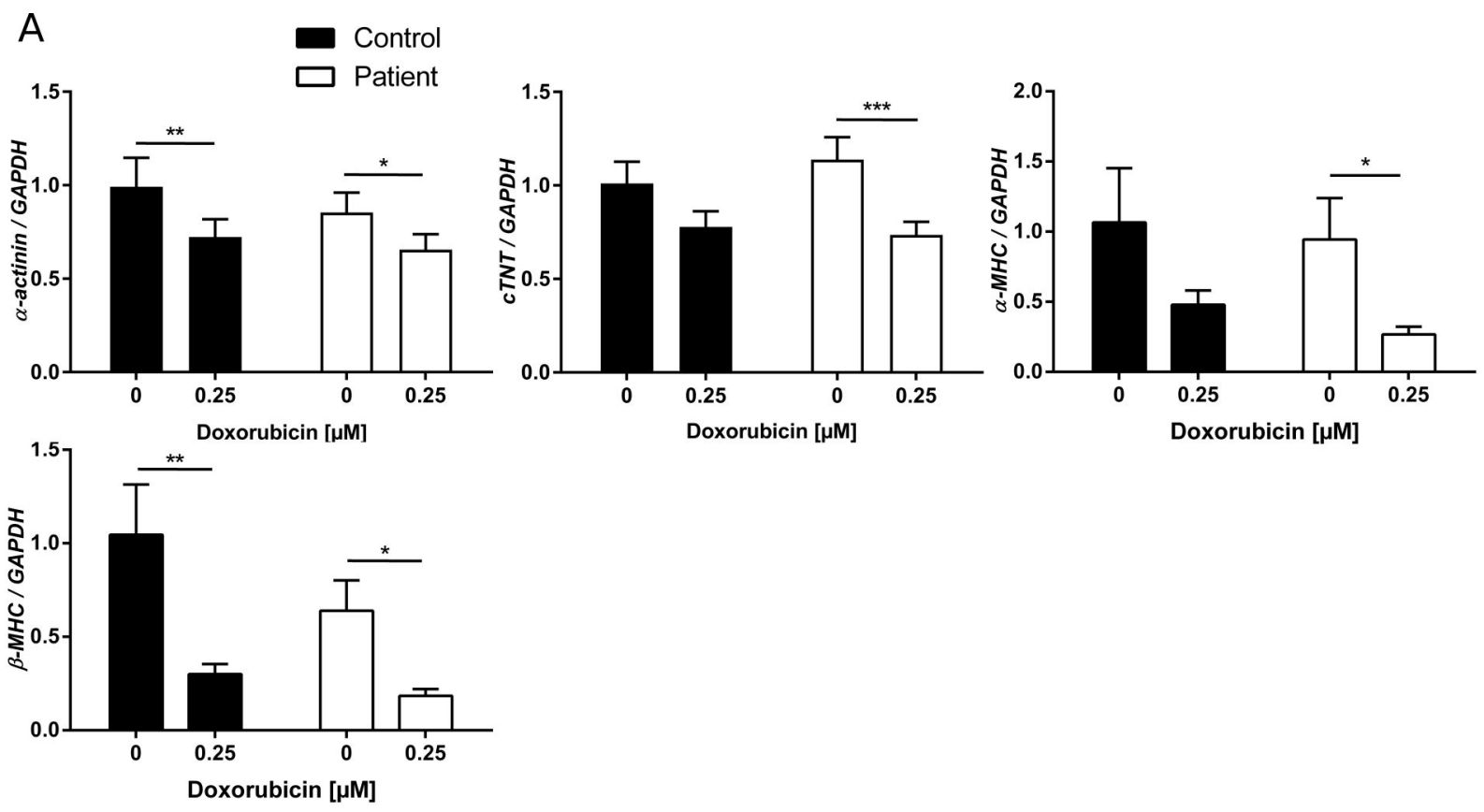

B

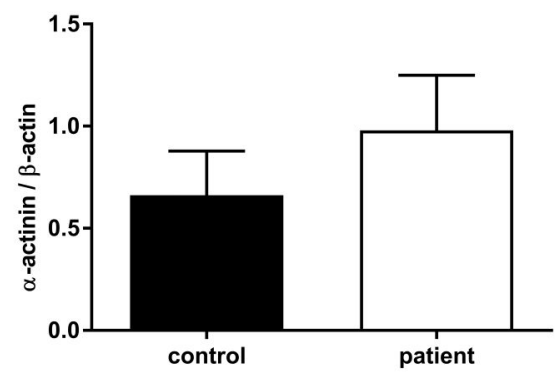

$\mathrm{D}$

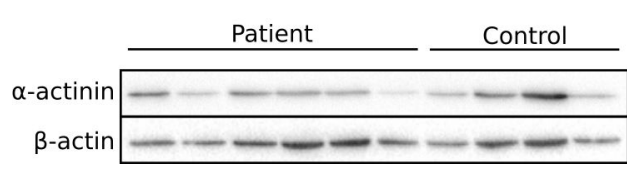

C
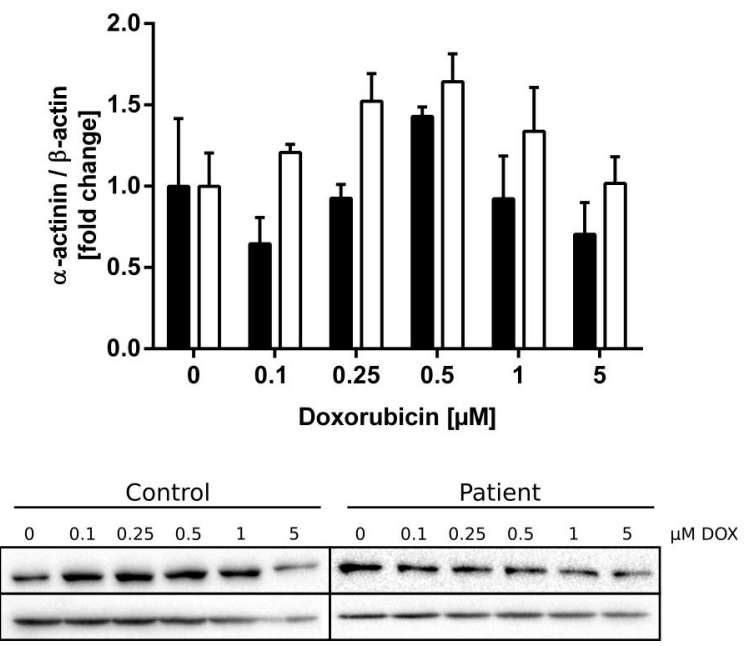

Figure 31: Gene expression analysis of sarcomeric proteins. A: Expression of $\alpha$-actinin, cTNT, $\alpha-M H C$ and $\beta-M H C$ on the mRNA level in iPSC-CMs of both groups. Sample number: 11 Ctrl-iPSC-CM differentiations, 18 ACT-iPSC-CM differentiations. B: $\alpha$-actinin in iPSC-CMs of both groups on the protein level. C: DOX-induced relative changes in the amount of $\alpha$-actinin in iPSC-CMs of both groups. D: Representative western blot results used for quantification. Left: Untreated. Right: DOX treated. Sample number: 4 Ctrl-iPSC-CM differentiations, 6 ACT-iPSC-CM differentiations. Mean + SEM. ${ }^{*} p<$ $0.05, * * \mathrm{p}<0.01, * * * \mathrm{p}<0.001$.

\subsubsection{Mechanical functionality}

To gain a better understanding of the influence of DOX on the functionality of iPSC-CMs in the ACT model, EHMs were generated. Hereby, the beating frequency, the beating regularity and the force of contraction could be analyzed. The generation of EHMs and experiments were done in cooperation with the institute of pharmacology and toxicology of the University Medical Center Göttingen by the group of Dr. med. Malte Tiburcy (Figure 32 and 33). 
A

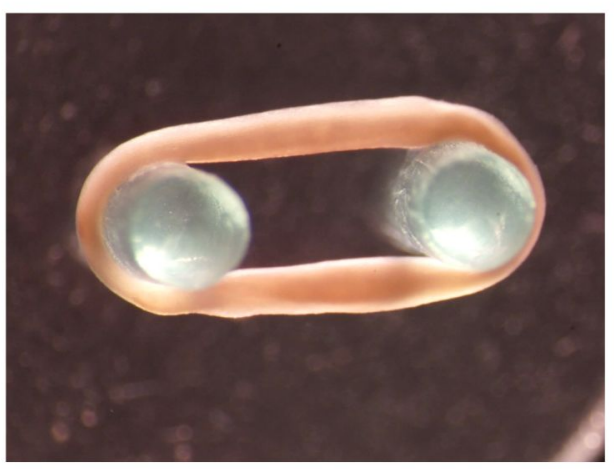

C

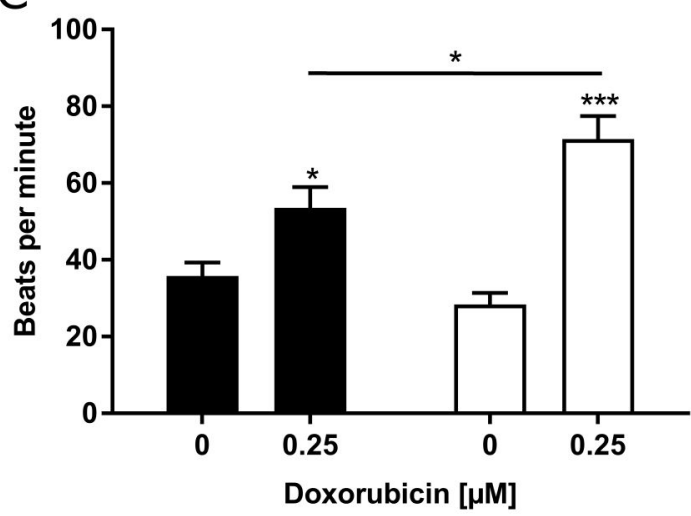

B

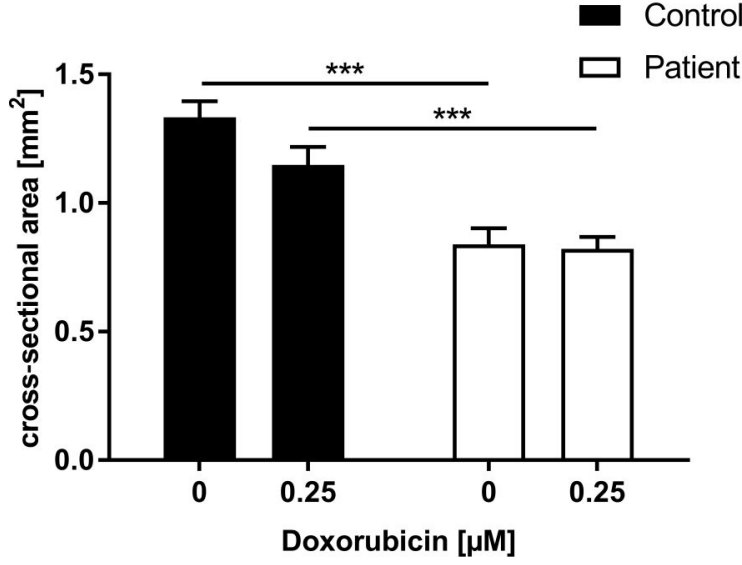

$\mathrm{D}$

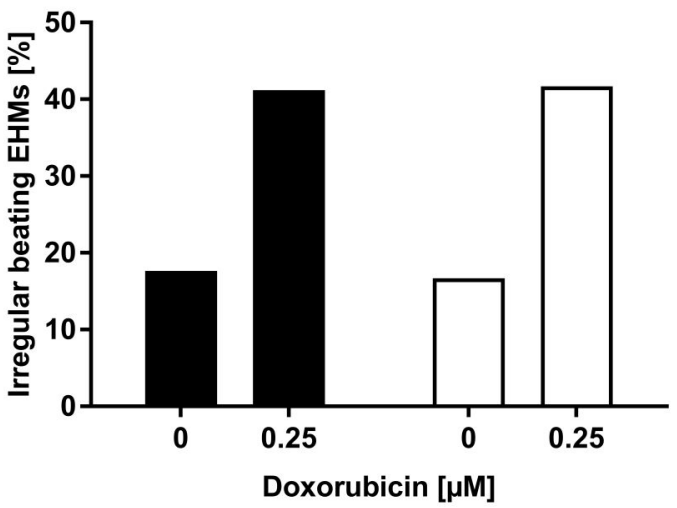

Figure 32: Generation of EHMs from Ctrl- and ACT-iPSC-CMs and beating activity dynamics. A: ACTEHM from Patient 2 mounted on dynamic silicon stretchers. B: Cross-sectional area of Ctrl- and ACTEHMs. C: Beating frequency of Ctrl- and ACT-EHMs. D: Amount of Ctrl- and ACT-EHMs that depict an irregular spontaneous beating activity. Sample number: 12 Ctrl-EHMs from 2 differentiations, 18 ACTEHMs from 3 differentiations. Mean + SEM. * $p<0.05, * * p<0.01, * * * p<0.001$.

Surprisingly, the cross-sectional area (CSA) of EHMs from the control group (Ctrl-EHMs) was significantly larger compared to the ACT patient group (ACT-EHMs). DOX treatment did not cause significant changes in either group (Figure $32 \mathrm{~B}$ ). EHMs of both groups showed beating activity with a comparable frequency of about 30 beats per minute. A significant DOXinduced increase in beating frequency was observed in both groups. This increase was significantly stronger in ACT-EHMs (Figure $32 \mathrm{C}$ ). About $15 \%$ of generated EHMs in both groups depicted irregular beating at basal conditions and DOX treatment caused the rate to increase to $40 \%$ (Figure $32 \mathrm{D}$ ). The force of contraction of EHMs was dependent on $\mathrm{Ca}^{2+}$ concentration in the measurement buffer (Figure $33 \mathrm{~A}$ ). The absolute maximal force generated by EHMs was comparable between both groups at basal conditions with about $1 \mathrm{mN}$. It was slightly decreased after DOX treatment in the control group. In ACT-EHMs, on the other hand, the DOX-induced decrease was significant (Figure $33 \mathrm{~A}, \mathrm{~B}, \mathrm{C}$ ). Interestingly, when set in relation to the CSA, ACT-EHMs generated significantly more force than Ctrl-EHMs at basal conditions (Figure $33 \mathrm{D}$ ). Furthermore, when set in relation to the CSA, DOX 
treatment did not change the relative force of Ctrl-EHMs, whereas it was significantly decreased in ACT-EHMs by about 35 \% (Figure 33 D, E). Taken together, EHMs from ACT-iPSCCMs showed a significantly decreased relative force after DOX treatment compared to CtrliPSC-CMs. 
A

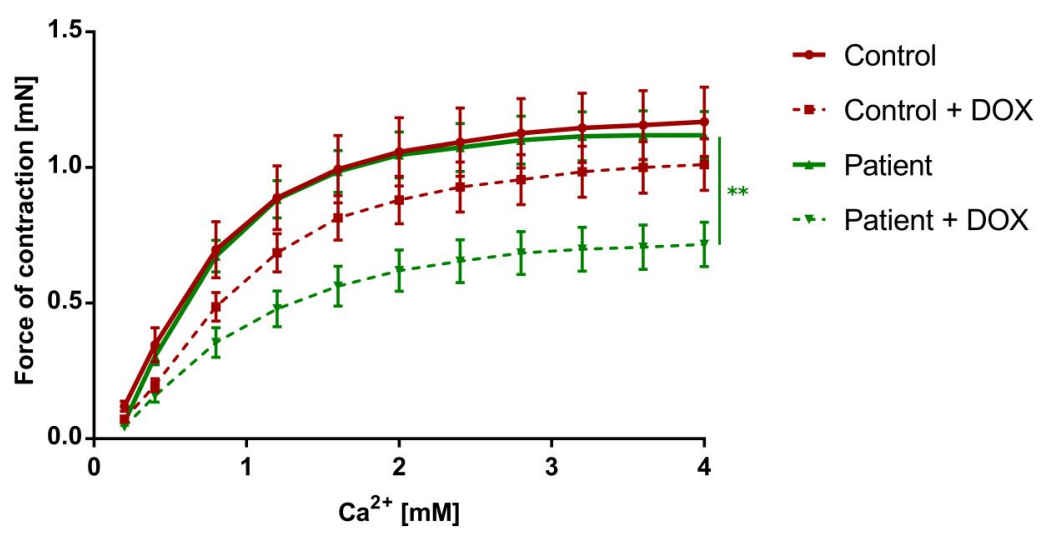

B

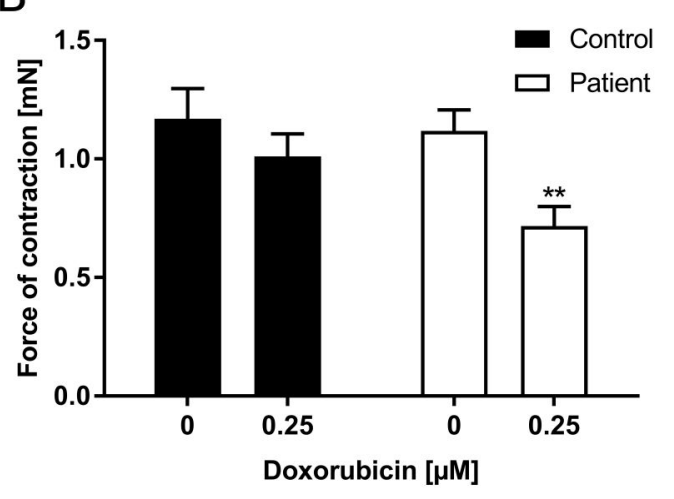

D

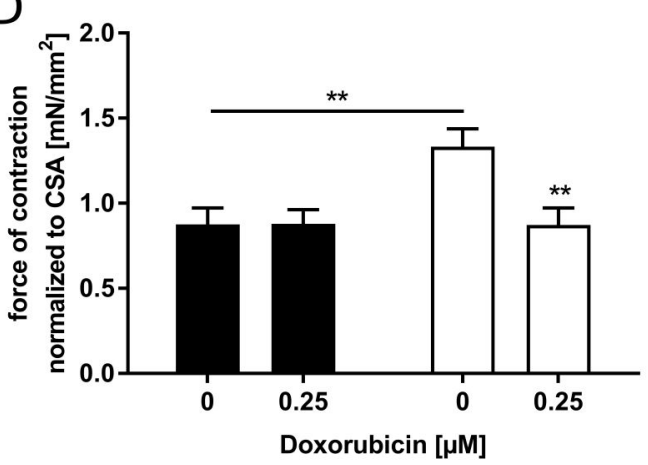

C

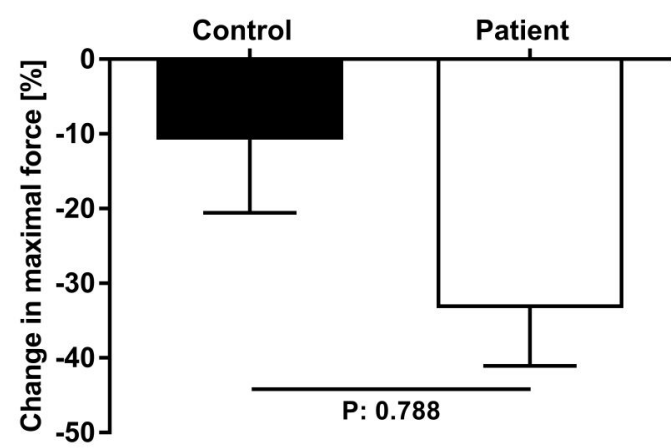

$E$

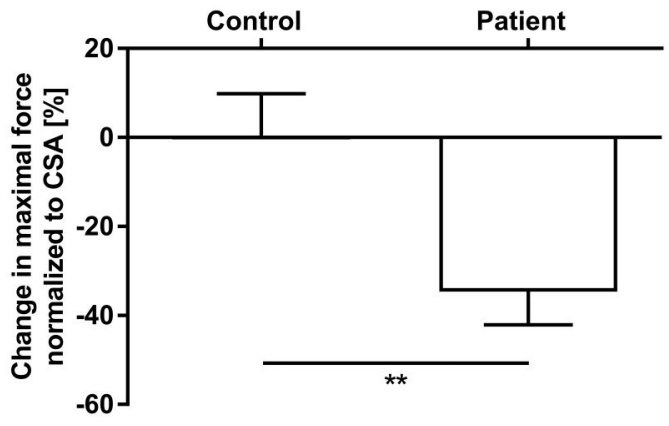

Figure 33: Analysis of Ctrl- and ACT-EHM force of contraction. A: $\mathrm{Ca}^{2+}$-dependent generation of contractile force of Ctrl- and ACT-EHMs. B: Maximal force of contraction of Ctrl- and ACT-EHMs. C: DOX-induced relative change of maximal contractile force of Ctrl- and ACT-EHMs. D: Maximal force of contraction of Ctrl- and ACT-EHMs relative to the EHM CSA. E: DOX-induced relative change of maximal contractile force of Ctrl- and ACT-EHMs relative to EHM CSA. Sample number: 12 Ctrl-EHMs from 2 differentiations, 18 ACT-EHMs from 3 differentiations. Mean + SEM. ${ }^{*} p<0.05,{ }^{* *} p<0.01$, $* * * p<0.001$. 


\section{Discussion}

The risk to develop ACT is a major limiting factor of anthracyclines such as DOX, which are otherwise very effective neoplastic drugs used against a broad range of solid and haematopoietic cancers. ACT is characterized by arrhythmia, bradycardia, tachycardia, decrease in LVEF and FS and irreversible CHF, all of which lead to increased morbidity and mortality. It is accompanied by histopathological changes such as myofibril loss, SR dilation, cytoplasmic vacuolization, swelling of mitochondria and increase in lysosome number. Despite being intensely used and studied for decades, the underlying DOX-induced pathomechanisms leading to ACT are still not fully understood. According to the current state of knowledge, ACT is a multifactorial syndrome with elevation of oxidative stress, poisoning of TOP2 $\beta$ and disturbances of $\mathrm{Ca}^{2+}$ homeostasis in CMs being key factors that all cause apoptosis. Additionally, a genetic predisposition for ACT has been suggested and several SNPs were statistically associated to ACT. Since human CMs are hard to obtain and culture, various animal models were used for studies of ACT. This may be a reason for contradicting results and poor outcomes in clinical trials. After a decade of establishment, the iPSC technology holds promise as a powerful tool to model ACT. Such a model may be used to investigate pathomechanisms and genetic predisposition in a human system, to screen for protectants and to develop patient-specific treatment.

In this work, a human iPSC-CMs based model of ACT was established using three ACT patients and two controls. ACT patients contained ACT-associated alleles of SNPs in the NADPH oxidase subunits encoding genes CYBA and RAC2, whereas the controls did not. In the first part of this work, integration-free hiPSCs were generated that expressed pluripotency markers on the mRNA and protein level and had the potential to develop cells of all three germ layers in vitro and in vivo. Subsequently, CMs were derived from ACT and control iPSCs, using a direct cardiac differentiation protocol with about $95 \%$ purity. iPSC-CMs expressed cardiac marker genes on the mRNA and protein level and depicted an organized sarcomeric structure.

In the second part of the present study, ACT-related parameters were analyzed in Ctrl- and ACT-iPSC-CMs upon DOX application, namely the gene expression of NADPH oxidases and $\mathrm{Ca}^{2+}$ handling proteins on the mRNA and protein level, the production of $\mathrm{ROS}, \mathrm{Ca}^{2+}$ signaling, apoptosis, the sarcomeric integrity and the overall mechanical functionality of iPSC-CMs. 


\subsection{Reprogramming and cardiac differentiation}

\subsubsection{Generation of hiPSCs from ACT patients and controls}

Pluripotency is induced and maintained by extracellular signaling pathways as well as intracellular transcriptional networks. These were stimulated and manipulated in this work. Integration-free hiPSCs were successfully generated by transient expression of OCT4, SOX2, KLF4 and C-MYC in dermal fibroblasts using the sendai virus system. The reprogramming efficiency was very high with $7.2 \%$. This is contrary to reported reprogramming efficiency of the CytoTune 2.0 kit of about 1.5 \% (Beers et al., 2015). The discrepancies likely arise from the different means to estimate reprogramming efficiency. In our study, alkaline phosphatase positive iPSC colonies were counted. The efficiency was calculated using the number of cells plated for reprogramming. However, Beers et al. state that some alkaline phosphatase positive colonies do not meet further criteria of pluripotency (Beers et al., 2015). Therefore, the authors only counted iPSC colonies that remained vital after passaging and expressed pluripotency markers. Taken together, in this work 24 and 16 iPSC lines were generated from three ACT patients and two control donors, respectively.

Prior to the use of hiPSCs in disease modeling, the generated cell lines have to be characterized to assess the applicability of the hiPSCs for future use. For example, hiPSCs that are only partially reprogrammed might not differentiate into the desired cell type sufficiently (Koyanagi-Aoi et al., 2013; Takahashi and Yamanaka, 2016). The cell lines, which were generated in this work, showed a typical hiPSC morphology (Figure 10). They grew in flat colonies with high cell density and distinct edges and were positive for alkaline phosphatase. Furthermore, an upregulation of the pluripotency-related genes NANOG, OCT4, LIN28, SOX2 and GDF3 was observed compared to the dermal fibroblasts used for reprogramming (Figure 11). Analyses on the protein level of LIN28, OCT4, NANOG, SOX2, SSEA4 and TRA-1-60 validated these results (Figure 12). This gene expression data suggests successful activation of the endogenous pluripotency network, since NANOG, LIN28, GDF3, SSEA4 and TRA-1-60 encoding genes are not part of the reprogramming vectors. Further indication of pluripotency was obtained by spontaneously differentiating the iPSC lines in vitro and analyzing the expression of tissue-specific markers from each germ layer. In all cell lines, the expression of endodermal AFP, mesodermal CTNT and ectodermal TH was found after prolonged differentiation, whereas NANOG expression was usually downregulated (Figure 13). Few cell lines showed germ layer-specific gene expression even at differentiation start. This is most likely caused by iPSCs spontaneously differentiating under culture conditions where they should remain undifferentiated. This may occur during cocultivation with MEFs because the feeder cell-dependent cultivation of iPSCs is not chemically defined. This unwanted spontaneous differentiation of iPSCS is influenced by the quality and passage 
number of MEF. In immunofluorescence analyses, endodermal AFP, mesodermal $\alpha$ SMA and ectodermal $\beta$-III-Tub were detected (Figure 14). Together, these results are a strong evidence for pluripotency. The teratoma formation assay is widely considered as the pluripotency assay with the highest strength (Zhang et al., 2008). When implanted into immunodeficient SCID mice, PSCs proliferate and differentiate into cells of all germ layers supported by factors of the local milieu and circulating factors to form mostly benign teratomas. In this work, the teratoma formation assay was applied to at least one cell line per donor and neuronal rosettes (ectoderm), muscle (mesoderm) and intestinal tissue (endoderm) was detected (Figure 15). This further underlines the pluripotency stage of the generated iPSCs in this work.

To determine the cardiac differentiation efficiency of the iPSCs, EBs containing beating clusters were counted and the cardiac differentiation efficiency was shown to be $8-21 \%$ (Table 3). This is consistent with a study in which fibroblast-derived hiPSCs at early passages differentiated into cardiac cells with an efficiency of 10.6 - 21.7\% (Streckfuss-Bömeke et al., 2013). hESCs have been reported to differentiate with an efficiency of about $10-25 \%$ into cardiac cells (He et al., 2003). In this work, a spontaneous in vitro differentiation protocol was used to determine the cardiac differentiation efficiency. The quantification may be problematic due to several steps of the protocol using EBs. Since cardiac progenitor cells develop at around day 2-8 in in vitro differentiation experiments with hiPSCs (Cao et al., 2012), the selection of EBs seems to be a critical step. It is not clear how the EB size and cell number is associated with the development of CMs. Furthermore, the differentiation might be influenced by the size and density of the used hiPSC colonies.

Taken together, the characterization of 10 cell lines suggests that the analyzed Ctrl- and ACTiPSCs were pluripotent without differences between both groups. Furthermore, they contain a sufficient cardiac differentiation potential. Hence, the generated iPSCs were suitable for cardiac differentiation and to model ACT.

\subsubsection{Generation of hiPSC-CMs from ACT patients and controls}

To derive large quantities of CMs from iPSCs with high purity, a direct cardiac differentiation protocol was used, which combines previously reported approaches (Burridge et al., 2014; Lian et al., 2012, 2013; Tohyama et al., 2013). The differentiation of iPSCs into CMs took place under chemically defined conditions with minimal lot-dependent influences, thereby ensuring a high comparability between differentiation experiments. In a recent report, iPSC$\mathrm{CMs}$ were cultured in medium supplemented only with ascorbic acid and human recombinant albumin for over 200 days (Burridge et al., 2014). In contrast to this, in our experiments this medium was suitable only for the initial differentiation steps. Prolonged cultivation of iPSC-CMs in this medium often caused cell detachment and death. In our work, 
supplementation with B27 attenuated these problems. iPSC-CMs were cultured for 2-3 month to enable maturation, which was previously suggested (Lundy et al., 2013). In our work, iPSC-CMs cultured for 2-3 month, expressed CM-specific genes, had a high sarcomeric organization and were often multinucleated. Furthermore, their beating frequency could be dynamically paced and they depicted $\mathrm{Ca}^{2+}$ transients, which reacted to $\beta$-adrenergic signaling by isoproterenol application (Figure 24). Taken together, these findings show that the iPSC$\mathrm{CMs}$ generated in this work were functional and reached a certain level of maturity. However, all currently described iPSC-CMs have embryonic to neonatal features and do not resemble adult CMs (Kolanowski et al., 2017; Yoshida and Yamanaka, 2017). In our work, the focus was not on the analysis of the level of iPSC-CM maturity. Hence, no direct comparisons were performed between iPSC-CMs and adult human CMs. The same differentiation protocol was used to successfully model takotsubo cardiomyopathy, which develops in adult humans underlining a certain maturity of the cells (Borchert et al., 2017). Several parameters were observed in our work that show immaturity of generated iPSC-CMs compared to adult human CMs (see appendix, Table 5). iPSC-CMs were proliferating, which is a typical characteristic of embryonic CMs. In the postnatal heart, the size is increased by hypertrophic growth of CMs (Yang et al., 2014b). The morphology of iPSC-CMs resembled embryonic CMs (Figure 16), which is multi-angled. Adult CMs depict a rod shape with a length to width ratio of about 7 and a lengths of about 130-140 $\mu \mathrm{m}$ (Kolanowski et al., 2017; Tracy and Sander, 2011). In adult CMs, sarcomeres are organized uniformly lengthwise. In the generated iPSCCMs, sarcomeres were often distributed multi-directionally or circularly (Figure 16). It is likely that the low expression of NADPH oxidase subunits, detected in this work, was also a consequence of immaturity of iPSC-CMs. To achieve higher degrees of maturation, various approaches have been suggested, including biochemical and physical stimulation and 3D culture techniques (Kolanowski et al., 2017). It is reasonable to suggest, that significant increase of overall iPSC-CM maturity requires combination of those approaches to simulate in vivo development.

\subsection{Modeling ACT with hiPSC-CMs from ACT patients and controls}

The human ACT model generated in this work consists of ps-hiPSC-CMs which were treated with DOX to trigger ACT-associated phenotypes. Accordingly, finding an appropriate DOXapplication protocol was crucial for the entire study. The patients of the RICOVER-60 trials were treated with six or eight cycles of DOX, as part of the CHOP regiment, with 14 days intervals (Pfreundschuh et al., 2008). The pharmacokinetics of DOX in human serum after intravenous injection is triphasic with successive half-lifes reached at about five minutes, one hour and 30 hours (Benjamin et al., 1977; Blaes et al., 2015; Robert and Gianni, 1993). Peak 
plasma concentrations of about $5 \mu \mathrm{M}$ and steady-state plasma concentrations of $25-250 \mathrm{nM}$ have been reported (Greene et al., 1983; Minotti et al., 2004). However, there is a great variance between individual DOX dosage and schedule. The different ACT pathomechanisms seem to be highly intertwined and it is hard to distinguish between phenotypes directly induced by DOX and secondary phenotypes, resulting from accumulating damage. For this reasons, it was important to use comparable conditions of DOX treatment for all analyses.

Ultimately, we decided to culture the ps-hiPSC-CMs in medium supplemented with DOX ranging from 0.1 to $5 \mu \mathrm{M}$ for 24 hours, thereby simulating the low serum elimination time and high range of concentration variety. We considered further treatment cycles and washout intervals to be unfeasible, since even more variables would be added. When especially time consuming or expensive assays where performed, only low and high DOX concentrations of about 0.25 and $5 \mu \mathrm{M}$ were included, representing steady-state and peak plasma concentrations, respectively. Interestingly, several independent research groups reported similar DOX-application protocols for hiPSC-CMs, thereby strengthening our rationale (Burridge et al., 2016; Chaudhari et al., 2016; Eldridge et al., 2014).

\subsubsection{NADPH oxidase}

To determine putative effects of ACT-associated SNPs and patient-specific phenotypes, the gene expression of NADPH oxidase subunits was determined on the mRNA and protein level. The overall expression of NADPH oxidase subunits on the mRNA level in iPSC-CMs of both groups was low, which was indicated by high CT values of qRT-PCR (see appendix, Table 5). Only RAC1 expression was comparable to the analyzed cardiac genes. NCF1, NCF2, NCF4 were not detectable, suggesting extremely low expression or inactivity of these genes in iPSC-CMs. To still ensure validity of results, qRT-PCR was performed with a high sample number (up to $n=17$ ). Similar to the concentration of $m R N A$, the amount of NADPH oxidase subunits NOX2, NOX4, p67phox, RAC2 and p22phox on the protein level was low and quality of western blots for p22phox and RAC2 were not sufficient for quantification. For these reasons, the results of NADPH oxidase subunit expression in our work have to be interpreted with caution and need to be validated by additional experiments.

The expression of NOX2, NOX4, CYBA and RAC1 on the mRNA level was comparable between iPSC-CMs from both groups without DOX treatment (Figure 17). The expression of RAC2 in ACT-iPSC-CMs was higher compared to the control group. However, this trend was not statistically significant. In parts, these findings are in line with a study in human whole blood leukocytes. The authors reported significantly higher expression of RAC2 and NCF4 in donors with the ACT-associated allele of RAC2 SNP rs13058338 (Schirmer et al., 2007). In our work, NOX2, NOX4 and CYBA expression was downregulated after DOX application in iPSC-CMs from both groups, whereas RAC1 was downregulated only in ACT-patients (Figure 17). This is 
contrary to a previous report where NOX2 and NOX4 are upregulated upon DOX application in mice heart tissue detected by qRT-PCR (Zhao et al., 2010). On the other hand, DOX application did not change mRNA levels of NCF1, NCF2 or CYBA in ventricular tissue of mice (Yoshizawa et al., 2016). Furthermore, NADPH oxidase expression in heart tissue is described to be upregulated in mice models of heart disease, such as heart failure, cardiac hypertrophy and myocardial infarction (Kuroda and Sadoshima, 2010; Looi et al., 2008; Zhang et al., 2013). Therefore, our findings on the mRNA level were surprising and the reasons for these discrepancies remain to be determined. Since we detected a downregulation of mRNA expression in most of our analyzed genes, we could not rule out an overall defect in transcription after DOX treatment in our cells.

Consistent with the findings on the mRNA level, the protein amount of NOX2, NOX4 and RAC1 were comparable in Ctrl- and ACT-iPSC-CMs without DOX treatment (Figure 18). Although p40phox encoding NCF4 expression was not detectable on the mRNA level, p40phox was observed on the protein level. In future experiments, the efficiency of NCF4 detection in iPSC-CMs via qRT-PCR should be optimized. Interestingly, p40hox amount was significantly higher in untreated ACT-iPSC-CMs than in Ctrl-iPSC-CMs. These findings are in line with the aforementioned study by Schirmer et al. (Schirmer et al., 2007). The authors suggested that the ACT-associated allele of RAC2 SNP rs13058338 may contribute to increased expression of NCF4, which encodes for p40phox. The DOX-induced downregulation of NOX2, NOX4, CYBA and RAC1 on the mRNA level was not observed on the protein level (Figure 18). Instead, p40phox and RAC1 were upregulated in the control and ACT patient group, respectively. To account for a possible delay between changes on the mRNA level and changes on the protein level, the amount of protein was determined 48 hours after single DOX application. The changes that were observed on the mRNA level were also not represented on the protein level after the 48 hour delay. Interestingly, the amount of p40phox was significantly reduced upon DOX treatment in Ctrl-iPSC-CMs but not in ACT-iPSC$\mathrm{CMs}$ (Figure 18). These findings suggest that p40phox expression may be regulated differently between iPSC-CMs of both groups under basal conditions as well as upon DOX application. The function of p40phox as part of the NADPH oxidase is somewhat controversial in the literature, since it has been described as both stimulating and inhibiting regulator of NADPH oxidase 2 (Bouin et al., 1998; Lopes et al., 2004; Sathyamoorthy et al., 1997; Someya et al., 1999). Strong evidence support the common assumption that binding of p67phox (encoded by NCF2) to NOX2 and active RAC1/2 is required for activation of NADPH oxidase 2. Upon phosphorylation, p47phox (encoded by NCF1) acts as an adapter protein and supports the binding of p67phox to NOX2 (Brandes et al., 2014). In the absence of p47phox, p40phox activates NADPH oxidase 2 expression (Fan et al., 2009; Tamura et al., 2007). However, it was also reported that phosphorylated p40phox inhibits p47phox-bound 
NADPH oxidase (Lopes et al., 2004). On the basis of their findings, Fan et al. hypothesize that dynamic phosphorylation of p40phox determines NADPH oxidase 2 activity on the basal level and upon stimulation. At basal conditions, p40phox is phosphorylated, contributes to basal NADPH oxidase 2 activity and inhibits p47phox phosphorylation. Upon agonist stimulation, p40phox gets rapidly dephosphorylated, enabling p47phox phosphorylation and increase in NADPH oxidase 2 activity. Rephosphorylation of p40phox finally contributes to full NADPH oxidase 2 activity (Fan et al., 2009). On the basis of the study of Fan et al., NADPH oxidase 2 in ACT-iPSC-CMs may be more active on the basal level due to the higher amount of p40phox. The phosphorylation status of p40phox in Ctrl- and ACT-iPSC-CMs needs to be elucidated in future experiments.

In conclusion, ACT-iPSC-CMs showed a higher amount of NADPH oxidase subunit p40phox compared to Ctrl-iPSC-CMs under basal conditions. This may be an effect of the RAC2 SNP rs13058338, as it has been previously suggested (Schirmer et al., 2007) and it may have an impact on NADPH oxidase 2 activity. Furthermore, p40phox regulation in response to DOX application differs between both groups, suggesting an important role of p40phox in ACT. However, the exact role of p40phox in the context of NADPH oxidase 2 activity is still unclear and needs to be analyzed in the future. To our knowledge, this is the first report of NADPH oxidase expression analyses in a patient-specific iPSC-CM ACT model.

\subsubsection{ROS generation}

In this work, a main focus was on the analysis of ROS generation in iPSC-CMs as part of modeling the ACT. Since the increase of oxidative stress is a key pathomechanism of ACT, we were interested in general DOX-induced changes on ROS levels. On the other hand, we also aimed to determine ACT patient- and control-specific ROS phenotypes in iPSC-CMs without and upon DOX treatment. The reason for this was that the recruited ACT patients contained ACT-associated SNPs in NADPH oxidase subunits. Therefore, a hypothesis of this work was that NADPH oxidase activity and subsequently the amount of ROS differs between both groups as a functional consequence of the ACT-associated SNPs. However, the accurate measurement and analysis of ROS can be a complex task due to their reactivity, ephemeral nature and similarities between different oxidative species. To measure and analyze ROS, the experimenter may choose from a wide variety of methodology, each having certain advantages and disadvantages (Dikalov et al., 2007; Griendling et al., 2016). $\cdot \mathrm{O}_{2}^{-}$and $\mathrm{H}_{2} \mathrm{O}_{2}$ are the most relevant ROS for the iPSC-CM ACT model, since they influence major pathways, are progenitors for further ROS and are generated by NADPH oxidase 2 and 4, respectively. For this purpose, assays were chosen that specifically detect $\cdot \mathrm{O}_{2}{ }^{-}$and $\mathrm{H}_{2} \mathrm{O}_{2}$.

The Amplex Red assay is commercially available and developed by Molecular Probes. It is considered a first grade method by the American Heart Association as it specifically detects 
$\mathrm{H}_{2} \mathrm{O}_{2}$ with a high sensitivity and allows for quantification (Griendling et al., 2016). In principle, the non-fluorescent Amplex Red is oxidized by horseradish peroxidase upon $\mathrm{H}_{2} \mathrm{O}_{2}$ binding to form the fluorescent molecule resorufin. Since horseradish peroxidase does not pass the cell membrane, only extracellular $\mathrm{H}_{2} \mathrm{O}_{2}$ is detected. However, it has also been suggested that $\mathrm{H}_{2} \mathrm{O}_{2}$ is diffusible and an equilibrium between cytoplasm and the cell's surrounding buffer may be reached (Dikalov et al., 2007). Using the Amplex Red assay, we found that ACT-iPSCCMs produce more $\mathrm{H}_{2} \mathrm{O}_{2}$ at every tested condition than Ctrl-iPSC-CMs, which was significant upon treatment with $0.5 \mu \mathrm{M}$ DOX (Figure $19 \mathrm{~A}$ ). Application of low DOX concentrations induced a significant increase of $\mathrm{H}_{2} \mathrm{O}_{2}$ in iPSC-CMs of both groups. No differences between Ctrl- and ACT-iPSC-CMs were found when the relative DOX-induced increase in $\mathrm{H}_{2} \mathrm{O}_{2}$ amount was compared (Figure $19 \mathrm{~B}$ ). Interestingly, application of high DOX concentrations did not change the $\mathrm{H}_{2} \mathrm{O}_{2}$ amount. A possible explanation for this phenomenon is based on the nature of the Amplex Red assay, which is dependent on the number of cells. Since iPSC-CMs of both groups were significantly apoptotic upon application of high DOX concentrations (Figure 29), the unchanged amount of detected $\mathrm{H}_{2} \mathrm{O}_{2}$ is likely due to a decrease of viable cells rather than actual production of $\mathrm{H}_{2} \mathrm{O}_{2}$ per cell. Importantly, the amount of $\mathrm{H}_{2} \mathrm{O}_{2}$ was still significantly increased seven days after single treatment with a low concentration of DOX in iPSC-CMs of both groups (Figure $19 \mathrm{C}$ ). Even 21 days later, iPSC-CMs of both groups depicted an $\mathrm{H}_{2} \mathrm{O}_{2}$ increase of about two-fold. These findings indicate that single DOX treatment for 24 hours induces chronic pathological changes in the ACT iPSC-CM model.

We focused on analyses using genetically-encoded, roGFP2-based biosensors. The redoxsensitive roGFP2 was originally generated by substituting surface facing residues of GFP2 with cysteins that form disulfide bridges after oxidation (Dooley et al., 2004). As a result, roGFP2 possesses two excitation maxima at about 400 and $490 \mathrm{~nm}$ when fluorescence emission is detected at $510 \mathrm{~nm}$. The amplitude of both excitation maxima depends on the oxidation state. Upon oxidation of the roGFP2 cystein residues, the intensity of the excitation maximum at $400 \mathrm{~nm}$ increases, whereas the one at $490 \mathrm{~nm}$ decreases. Reduction causes the opposite effect. As a result, the oxidation state of roGFP2 can be analyzed ratiometrically, by detecting fluorescence emission at $510 \mathrm{~nm}$ upon excitation at both 400 and $490 \mathrm{~nm}$ with short time intervals. Ratiometric analyses offer great advantages over usual photometric methods, because artificial effects caused by photobleaching or differences in detector expression level can be neglected. roGFP2 offers dynamic and fast detection of oxidation. It is not affected by $\mathrm{pH}$ changes in the physiological range of $5.5-8.0$. RoGPF2 detects the oxidation state of the glutathione redox couple (reduced: GSH; oxidized: GSSG) which is mediated by glutaredoxins ( $G r x$ ) but may also be oxidized by other mechanisms (Dooley et al., 2004; Meyer et al., 2007). In this work, the constructs roGFP2-Orp1 and Grx1-roGFP2 were used, which contain roGFP2 fused to oxidant receptor protein-1 (Orp1) or glutaredoxin- 
1 (Grx1), respectively, thereby influencing the specificity of the construct. Both constructs locate to the cytoplasm and thereby detect intracellularly. However, the signals are not exclusively cytosolic, since a nuclear localization was also observed (Figure 20).

Orp1 is a peroxidase, which is highly selective for $\mathrm{H}_{2} \mathrm{O}_{2}$. Upon activation, it transmits oxidation to the transcription factor Yap1 (Delaunay et al., 2002). Interestingly, fusion of Orp1 to roGFP2 causes Orp1 to transmit the $\mathrm{H}_{2} \mathrm{O}_{2}$ specific oxidation to roGFP2 instead of Yap1. Thereby, roGFP2 is converted into a highly specific $\mathrm{H}_{2} \mathrm{O}_{2}$ detector. roGPF2-Orp1 only allows for relative comparisons and no absolute $\mathrm{H}_{2} \mathrm{O}_{2}$ concentrations can be determined. OxD of roGFP2-Orp1 represents the amount of $\mathrm{H}_{2} \mathrm{O}_{2}$. Although roGFP2-fusion proteins are not included in the official statement by the American Heart Association, roGPF2-Orp1 offers similar advantages as HyPer, is equally sensitive and additionally is not influenced by $\mathrm{pH}$ changes in the physiological range. Therefore, the value of analyses with roGPF2-Orp1 should be comparable to the first grade HyPer analyses (Griendling et al., 2016; Gutscher et al., 2009). Using roGFP2-Orp1 in iPSC-CMs, we found the intracellular $\mathrm{H}_{2} \mathrm{O}_{2}$ amount to be comparable between ACT patients and controls at basal conditions (Figure 22). Upon DOX application, a dose-dependent increase of $\mathrm{H}_{2} \mathrm{O}_{2}$ was observed in both groups, which was significant at 0.75, 1 and $5 \mu \mathrm{M}$ DOX. Upon DOX treatment with $0.5 \mu \mathrm{M}$, the increase of intracellular $\mathrm{H}_{2} \mathrm{O}_{2}$ was about $60 \%$ in both groups (Figure 22). The increase of extracellular $\mathrm{H}_{2} \mathrm{O}_{2}$ at the same DOX concentration was about $60 \%$ in controls and $90 \%$ in ACT patients, detected with Amplex Red (Figure 19). These data indicate comparability between both assays at low DOX concentrations. Since roGFP2-based measurements are ratiometric, a decrease in cell number, caused by DOX-induced apoptosis, does not influence the detection of $\mathrm{H}_{2} \mathrm{O}_{2}$. For this reason, no artificial decrease in intracellular $\mathrm{H}_{2} \mathrm{O}_{2}$ amount was observed, as it was likely the case for the Amplex Red assay (Figure 19 and Figure 22). This would explain the discrepancies between both assays upon application of higher DOX concentrations.

By fusing roGFP2 to Grx1, the construct's specificity towards GSSG is greatly enhanced (Gutscher et al., 2008). GSH is a tripeptide and belongs to the most important antioxidants in animals. Because of its high abundance, the oxidation state of the redox pair GSH/GSSG is traditionally used to reflect the overall redox state of the cell or the amount of oxidative stress, although these terms are poorly defined (Schafer and Buettner, 2001). Grx1-roGFP2 detects the GSH redox potential $\left(\mathrm{E}_{\mathrm{GSH}}\right)$, which is determined by the OxD of GSH/GSSG and the total amount of GSH. In this work, $\mathrm{E}_{\mathrm{GSH}}$ and OxD of Grx1-roGFP2 were oxidized more in ACTiPSC-CMs than in Ctrl-iPSC-CMs. The same was found upon application of DOX at every tested concentration, which was significant at $0.5 \mu \mathrm{M}$ (Figure $22 \mathrm{C}$ ). No differences between Ctrl- and ACT-iPSC-CMs were found when the relative DOX-induced increase in $\mathrm{H}_{2} \mathrm{O}_{2}$ amount was compared (Figure $22 \mathrm{D}$ ). No clear dose-dependency was observed in both groups, although the DOX-induced change of $\mathrm{E}_{\mathrm{GSH}}$ at $5 \mu \mathrm{M}$ was about $+6 \mathrm{mV}$ (Table 4). This amounts 
for about 40\% increase of OxD (Figure $22 \mathrm{D}$ ). In untreated iPSC-CMs of controls and ACT patients the mean $\mathrm{E}_{\mathrm{GSH}}$ was -308.15 and $-301.46 \mathrm{mV}$, respectively (Table 4). Although there is no clear consensus in the literature about cytosolic $E_{G S H}$ due to varying methodology, this data is consistent with other studies. For example, a cytosolic $E_{G S H}$ of $-289 \mathrm{mV}$ was found in hESC-CMs using Grx1-roGFP2 (Heta, 2017). Further studies were employed with genetically encoded fluorescent probes in different organisms. A cytosolic $E_{G S H}$ of -320 to $-310 \mathrm{mV}$ and $-289 \mathrm{mV}$ was reported in the yeast S. cerevisiae (Morgan et al., 2011; Østergaard et al., 2004), and $-320 \mathrm{mV}$ in the tobacco plant (Brach et al., 2009). Additionally, a general estimation for the cytosolic $E_{G S H}$ of -320 to $-300 \mathrm{mV}$ in unstressed cells has been suggested (Meyer and Dick, 2010). However, a more oxidized cytosolic $\mathrm{E}_{\mathrm{GSH}}$ of about $-256.5 \mathrm{mV}$ (mean of two mouse lines) was reported in isolated mouse CMs (Swain et al., 2016).

Taken together, in this work the extracellular $\mathrm{H}_{2} \mathrm{O}_{2}$ amount (Amplex Red), the intracellular $\mathrm{H}_{2} \mathrm{O}_{2}$ amount (roGFP2-Orp1) and the cytosolic $\mathrm{E}_{G S H}$ (Grx1-roGFP2) was analyzed. DOX caused a dose-dependent increase in intra- and extracellular $\mathrm{H}_{2} \mathrm{O}_{2}$ in iPSC-CMs. These results show that a key pathomechanism of ACT, namely the DOX-induced increase in ROS, can be modeled using iPSC-CMs. Our findings are in line with the study of Burridge et al. who show that ROS increases dose-dependently in iPSC-CMs from ACT patients and controls upon DOX application (Burridge et al., 2016). Since the ACT-associated variants of SNPs in the ACT patients are linked to NADPH oxidases, a hypothesis of this work was that ROS production would differ between ACT patients and controls. On basal level, we found a higher extracellular $\mathrm{H}_{2} \mathrm{O}_{2}$ amount and a more oxidized $\mathrm{E}_{G S H}$ in ACT-iPSC-CMs compared to Ctrl-iPSCCMs. Furthermore, the extracellular $\mathrm{H}_{2} \mathrm{O}_{2}$ amount and $\mathrm{E}_{\mathrm{GSH}}$ was significantly higher in ACTiPSC-CMs than in Ctrl-iPSC-CMs upon treatment with $0.5 \mu \mathrm{M}$ DOX. These findings support the hypothesis that NADPH oxidase activity is higher in ACT patients with ACT-associated SNPs than in controls. Burridge et al. reported that the amount of ROS in iPSC-CMs under basal conditions is comparable between ACT patients and controls (Burridge et al., 2016). To analyze ROS, the authors used a commercially available general ROS detector called CellROX (Thermo Fisher Scientific), whose molecular formula is not published. Furthermore, in the study of Burridge et al. the commercially available $\mathrm{H}_{2} \mathrm{O}_{2}$ detector ROS- $\mathrm{Glo}_{2} \mathrm{H}_{2}$ (Promega) was used, which has not yet been critically assessed in the literature.

In our work, the DOX-induced relative change of ROS in iPSC-CMs was comparable between controls and ACT patients using Ampex Red, roGFP2-Orp1 and Grx1-roGFP2 (Figure $19 \mathrm{~B}$ and Figure $22 \mathrm{~B}, \mathrm{D}$ ). These findings are contradicting the work of Burridge et al. who reported a stronger DOX-induced increase of ROS production in iPSC-CMs from ACT patients, compared to controls (Burridge et al., 2016). Because the relative increase of ROS was the same in Ctrland ACT-iPSC-CMs, our findings favor the hypothesis of Zhang et al., which states that generation of ROS is not a direct cause of ACT and may not be directly induced by DOX 
(Zhang et al., 2012). Zhang et al. show that TOP2 $\beta$ mediates DOX-induced downregulation of genes involved in regulation of mitochondrial biogenesis and function. Subsequently, impairment of mitochondria results in the generation of ROS which was reduced by $70 \%$ in TOP2 $\beta$ depleted mice.

As discussed in detail in chapter 5.2.2.1, several other methodologies have been attempted in our work to detect ROS that are directly generated by the NADPH oxidase. Thereby, we aimed to assess the activity of the NADPH oxidase. Unfortunately, these approaches were not successful. As a result, the NADPH oxidase activity could not be directly determined in iPSC-CMs in our work. This is because the ROS that was detected in our work could have been generated by additional sources besides the NADPH oxidase. Hence, we could not determine the functional consequences of the ACT-associated SNPS on the activity of the NADPH oxidase.

The results of this work show that roGFP2 based biosensors were functional in our experimental setup and responded to reduction and oxidation in a dynamic way. However, both biosensors located in the entire cytoplasm and nucleus. The Amplex Red assay detects extracellular $\mathrm{H}_{2} \mathrm{O}_{2}$. For this reasons, it is possible that ACT patient-specific phenotypes were not detected that are restricted to certain compartments, such as mitochondria or the SR, or microdomains as the NADPH oxidase environment. Therefore, DOX-induced ROS production in iPSC-CMs should be analyzed in organelles and microdomains to determine patientspecific patterns. For this purpose, genetically encoded ROS detectors may be used, which are fused to proteins with a distinct localization. For example, roGFP has been fused to p47phox, which is part of the NADPH oxidase 2 activating complex, thereby locating roGFP in close proximity to the NADPH oxidase (Pal et al., 2013). Using this fusion protein p47-roGFP, the NADPH oxidase activity was detected in macrophages. However, it was also shown that p47phox activity was still mediated by p47-roGFP. Hence, p47-roGFP expression likely causes an artificial activation of NADPH oxidase. Consequently, p47-roGFP is not suitable to analyze the activity of NADPH oxidase in the iPSC-CM ACT model. Nevertheless, similar sensor constructs may be generated to measure ROS in NADPH oxidase microdomains without artifacts. Furthermore, genetically encoded ROS biosensors have been described, which locate to specific organelles such as mitochondria, the ER or the nucleus (Bilan and Belousov, 2017).

An alternative approach to analyze the patient-specific NADPH oxidase activity and functional effects of ACT-associated SNPs may be the stimulation of NADPH oxidase. Angiotensin II was shown to activate NADPH oxidase 2 (Wagner et al., 2014). Alternatively, the use of recently described inhibitors, which are specific against NADPH oxidase may contribute to the determination of NADPH oxidase generated ROS (Altenhöfer et al., 2015). In this work it was not feasible to perform additional experiments with further conditions 
such as application of angiotensin II or NADPH oxidase inhibitors. However, additional specific activation or inhibition of the NADPH oxidase may reveal patient-specific phenotypes which are otherwise concealed.

In conclusion, the amount of extracellular $\mathrm{H}_{2} \mathrm{O}_{2}$ at basal conditions was higher and cytosolic $\mathrm{E}_{\mathrm{GSH}}$ was more oxidized in ACT-iPSC-CMs than in Ctrl-iPSC-CMs. Our findings show, that ROS production is increased in iPSC-CMs upon DOX treatment, which is a key pathomechanism of ACT and thereby highlight the applicability of iPSC-CMs for modeling ACT. Significantly higher amounts of ROS were observed in ACT-iPSC-CMs compared to Ctrl-iPSC-CMs upon treatment with $0.5 \mu \mathrm{M}$ DOX.

\subsubsection{Alternative approaches for ROS analysis}

First trials of the 2',7'-dichlorodihydrofluorescein diacetate $\left(\mathrm{H}_{2} \mathrm{DCF}-\mathrm{DA}\right)$ assay in iPSC-CMs in this work showed increased amounts of ROS after treatment with $0.1 \mu \mathrm{M}$ DOX for 24 hours (see appendix, Figure 38). $\mathrm{H}_{2}$ DCF-DA is a commonly used dye to measure intracellular ROS in living cells. In theory, the cell permeable $\mathrm{H}_{2}$ DCF-DA is cleaved by intracellular esterases to $2^{\prime}, 7^{\prime}$-dichlorodihydrofluorescein, which is not cell permeable and thereby trapped within the cell. Oxidation by ROS produces the fluorescent molecule 2',7'-dichlorofluorescein (DCF), which can be analyzed. However, the $\mathrm{H}_{2}$ DCF-DA assay is often criticized and accumulating evidence suggest that it should not be used for accurate analyses. $\mathrm{H}_{2} \mathrm{DCF}$ is not a specific indicator for a certain ROS but is oxidized by $\mathrm{H}_{2} \mathrm{O}_{2}$, peroxynitrite, lipid hydroperoxide and $\cdot \mathrm{O}_{2}{ }^{-}$ (Dikalov et al., 2007). Furthermore, $\mathrm{H}_{2}$ DCF itself may cause the generation of $\cdot \mathrm{O}_{2}^{-}$by photoreduction and redox cycling. It may be oxidized by peroxidases and its oxidation by ROS is indirect, requiring transition metals and heme enzymes (Bonini et al., 2006; Rota et al., 1999a, 1999b).

The lucigenin-enhanced chemiluminescence assay is the most commonly used chemiluminescence-based methodology to detect $\cdot \mathrm{O}_{2}{ }_{2}^{-}$, among others such as luminol, $\mathrm{L}-012$ or coelenterazine. In principle, lucigenin reacts with $\mathrm{O}_{2}^{-}$to form an energy-rich product, which emits a photon. In this work, we aimed to determine the NADPH oxidase activity in iPSC-CMs by detecting generated $\mathrm{O}_{2}^{-}$using lucigenin in cell homogenates after stimulation with NADPH. In first trials, the luminescence signal significantly increased upon NADPH application but not upon additional treatment with the $\mathrm{O}_{2}{ }^{-}$scavenger tiron (see appendix, Figure 39). Additionally, specific enzyme inhibitors were applied, to determine the source of $\cdot \mathrm{O}_{2}^{-}$. Diphenyleneiodonium (flavoprotein inhibitor), VAS2870 (NADPH oxidase inhibitor), NGnitro-L-arginine methyl ester (nitric oxide synthase inhibitor), oxipurinol (xanthine oxidase inhibitor) and rotenone (mitochondrial electron chain inhibitor) were used. The application of these inhibitors suggested that the source of the signal was the NADPH oxidase (see appendix, Figure 39). Unfortunately, a poor reproducibility was observed between different 
experiments. Furthermore, no feasible means of normalization could be established, which is necessary to compare results of different conditions and donor groups. As a consequence of the poor reproducibility and comparability of the assay, further analyses were canceled. The validity of this assay has been questioned, since potential redox cycling of lucigenin and oxygen may produce $\cdot \mathrm{O}_{2}^{-}$(Vásquez-Vivar et al., 1997). However, in studies that report this redox cycling, the assay was often performed under artificial conditions (Dikalov et al., 2007). It is now mostly accepted, that redox cycling does not contribute significantly to the detected signal if the assay is set up correctly (Dikalov et al., 2007; Griendling et al., 2016; Li et al., 1998; Skatchkov et al., 1999). Much insight into the involvement of $\mathrm{O}_{2}^{-}$production in diseases such as atherosclerosis, hypertension, diabetes and heart failure was gained using the lucigenin-enhanced chemiluminescence assay which was validated by the use of other methodologies (Dikalov et al., 2007). On the other hand, the validity of NADPH oxidase activity determination using the lucigenin-enhanced chemiluminescence assay upon NADPH stimulation has recently been questioned (Rezende et al., 2016).

Whereas the lucigenin-enhanced chemiluminescence assay is considered a third grade assay by the American Heart Association, detection of $\mathrm{O}_{2}^{-}$by electron paramagnetic resonance (EPR) is classified as first grade and is the most accepted method (Griendling et al., 2016). EPR detects the absorption of microwave energy on the transition of unpaired electrons in an applied magnetic field (Dikalov et al., 2007). By using molecules called spin traps, specific radicals such as $\mathrm{O}_{2}^{-}$can be identified and quantified in a biological sample like iPSC-CMs. Apart from being the most accurate way to detect $\cdot \mathrm{O}_{2}{ }^{-}$, EPR analyses of iPSC-CMs are also expensive, since a large amount of cells are necessary $\left(1.5 \times 10^{6}\right.$ cells per condition). Additionally, advanced training is needed to operate the space consuming EPR devices and to analyze the data. In this work, we aimed to detect $\mathrm{O}_{2}^{-}$generated in iPSC-CMs upon DOX application using EPR as part of a collaboration with Dr. med. Andreas Petry and Prof. Agnes Görlach from the German Heart Centre, Munich (data not shown). In the first trial, $\cdot \mathrm{O}_{2}^{-}$was detected in iPSC-CMs and reduced by superoxide dismutase. However, these results were not reproducible in subsequent experiments. Unfortunately, further analyses were made impossible, due to technical failure of the EPR device at the partner site in Munich.

An alternative approach to detect $\cdot \mathrm{O}_{2}^{-}$is the use of dihydroethidium (DHE) which specifically reacts with $\cdot \mathrm{O}_{2}{ }^{-}$to form the fluorescent 2-hydroxyethidium. However, other ROS and enzymes react with DHE to form ethidium which is also fluorescent and not distinguishable from 2hydroxyethidium. Furthermore, the reaction products of DHE only exhibit fluorescence upon binding of DNA. Since DOX has similar fluorescent properties as 2-hydroxyethidium and also intercalates into DNA, this assay could not be used in the iPSC-CM ACT model. However, subsequent separation and detection of DHE, 2-hydroxyethidium and ethidium by highperformance liquid chromatography (HPLC) provides a promising means to specifically 
analyze $\cdot \mathrm{O}_{2}^{-}$in iPSC-CMs upon DOX treatment (Griendling et al., 2016). Applying the correct conditions such as inhibition of further $\mathrm{O}_{2}$ producing enzymes would make it possible to analyze the activity of NADPH oxidase. The probe MitoSOX is designed to detect $\cdot \mathrm{O}_{2}^{-}$in mitochondria. Since it is chemically similar to DHE the aforementioned restrictions also apply for MitoSOX, rendering it impractical for quantitative analyses in the ACT iPSC-CM model without further combination with HPLC (Zielonka and Kalyanaraman, 2010).

Genetically encoded biosensors such as HyPer are the best means to analyze intracellular $\mathrm{H}_{2} \mathrm{O}_{2}$ since they offer distinct advantages (Griendling et al., 2016). Hyper is a hybrid between the bacterial $\mathrm{H}_{2} \mathrm{O}_{2}$ sensor OxyR and the circularly permuted yellow fluorescent protein (Belousov et al., 2006). It is highly sensitive and specifically detects $\mathrm{H}_{2} \mathrm{O}_{2}$ without artificial ROS production. Contrary to means to detect ROS already mentioned, HyPer reacts dynamically. As a result, fast changes in both directions in the $\mathrm{H}_{2} \mathrm{O}_{2}$ amount can be observed. In this work, iPSC-CMs were transfected with HyPer3 (Bilan et al., 2013), an improved version of HyPer, using common transfection reagents at different conditions (data not shown). Unfortunately, the transfection efficiency was very poor with only few cells showing fluorescence. The fluorescence could not be detected with a microplate reader. In future work, HyPer3 analyses of single DOX-treated iPSC-CMs should be performed using a fast switching monochromator and fluorescence microscopy.

\subsubsection{Calcium Signaling}

Dysregulation of $\mathrm{Ca}^{2+}$ signaling is connected to a plethora of heart conditions, including arrhythmia, systolic and diastolic dysfunction, hypertrophy and heart failure (Eisner et al., 2017; Røe et al., 2015). Importantly, DOX has been shown to interact with key players of calcium signaling and also to alter their gene expression, thereby causing impaired $\mathrm{Ca}^{2+}$ homeostasis. This is suggested to be a pathomechanism of ACT. In this work, $\mathrm{Ca}^{2+}$ transients were analyzed using the fluorescent dye Fluo-4. The transient kinetics are a direct indicator of $\mathrm{Ca}^{2+}$ channel activity. ISO was used as a positive control and additional activator of $\beta$ adrenergic signaling. As expected, no differences were found between untreated Ctrl- and ACT-iPSC-CMs in transient rise time and amplitude. The transient decay time was significantly faster in ACT-iPSC-CMs. ISO caused a fastening of transient rise and decay time in both groups, indicating a good functionality of the system (Figure 24).

Previous in vitro studies in cardiac and skeletal muscle suggest that anthracyclines may stimulate as well as inhibit $\mathrm{Ca}^{2+}$ release from the SR (Abramson et al., 1988; Olson et al., 2000; Pessah et al., 1990). Interestingly, in our work a biphasic effect of DOX on the transient rise time and amplitude was found in iPSC-CMs of both groups. A low DOX concentration caused a significant decrease in rise time and increase of amplitude, whereas a high concentration caused no changes in rise time and a significant decrease of amplitude (Figure 
24). Both parameters are mainly determined by RYR2 activity. These data suggest that DOX activates RYR2 at low concentrations, whereas it inhibits RYR2 at high concentrations in iPSCCMs. Recent findings from Hanna et al. support this data (Hanna et al., 2014). Here, the authors examined the channel open probability of single RYR2 channels in a planar bilayer system upon treatment with different DOX concentrations. They found a RYR2 activation by DOX concentrations of up to $0.5 \mu \mathrm{M}$ which was maintained for the lifetime of the experiment. However, following an initial activation, a sustained RYR2 inhibition was found upon application of 1 and $2.5 \mu \mathrm{M}$ DOX and the activity was reduced to 81 and $48 \%$, respectively. The activation upon low DOX concentrations was reversible after drug wash-out, whereas the inhibition upon high concentrations was not reversible but preventable by preincubation with the reducing agent DTT. The authors concluded that DOX may activate RYR2 by ligand binding and inhibit it by oxidation of thiol residues. Similar effects of the DOXrelated anthracycline daunorubicin have been reported (Hanna et al., 2011). In our work, Ctrl-iPSC-CMs depicted a significantly stronger activation than ACT-iPSC-CMs upon treatment with a low amount of DOX, whereas no differences between both groups were observed at a high DOX concentration (Figure 24). Presupposing that our data and those of Hanna et al. are based on the same mechanisms, these findings suggest that RYR2 from controls may be more prone to DOX binding than those from ACT patients but that both are equally modified at thiol groups. This would be a novel hypothesis that requires further validation and explanation.

DOX induced a significant decrease in transient decay time at low and high concentrations in both Ctrl- and ACT-iPSC-CMs (Figure 24). This parameter of $\mathrm{Ca}^{2+}$ signaling is determined by SERCA and NCX activity. Hwang et al. compared hiPSC-CMs generated by different laboratories and showed that SERCA contributes $61 \%$ and $\mathrm{NCX} 31 \%$ to the decay of $\mathrm{Ca}^{2+}$ transients in iPSC-CMs. This is comparable to human and rabbit CMs (Hwang et al., 2015; Piacentino et al., 2003). Our findings therefore suggest an increase in activity of one or both channels. Unlike transient rise time and amplitude, the transient decay times were decreased to a comparable level in both groups. Since the decay times in untreated ACT-iPSC-CMs were significantly lower, the relative DOX-induced changes were significantly stronger in Ctrl-iPSCCMs compared to ACT-iPSC-CMs (Figure 24 C, D). In contrast to our data, other studies report anthracyclines and their metabolites to compromise SERCA function by direct binding (Cusack et al., 1993; Hanna et al., 2014).

Accumulating evidence suggests that influences of anthracyclines on $\mathrm{Ca}^{2+}$ channels ultimately result in a decrease of SR content and increase in cytosolic $\mathrm{Ca}^{2+}$ concentration (Kim et al., 2006; Sag et al., 2011). Unfortunately, diastolic $\mathrm{Ca}^{2+}$ concentration could not be analyzed in this work due to limited time and work capacity. Quantitative $\mathrm{Ca}^{2+}$ analyses would have required the use of a different $\mathrm{Ca}^{2+}$ sensitive dye, a different experimental setup and complex 
standardization for every measurement. For the future, analyses of diastolic calcium with this ACT model seem promising.

As discussed above, the observed DOX-induced changes in $\mathrm{Ca}^{2+}$ transient properties might result from direct interactions of key proteins with DOX or from their oxidation by increased amount of ROS. However, changes in their gene expressions upon DOX treatment have also been reported and may contribute to impaired $\mathrm{Ca}^{2+}$ handling. Furthermore, phosphorylation by PKA and CamKII regulates the activity of RYR2 and PLN (and thereby SERCA). For these reasons, the expression of key proteins was analyzed on the mRNA and protein level. The amount of SERCA, PLN, NCX and CamKII mRNA was comparable in Ctrl- and ACT-iPSC-CMs under basal conditions (Figure 25). RYR2 was expressed about twice as high in control patients. Upon treatment with a low DOX concentration, significant downregulation of $R Y R 2$ and SERCA was found. This is in agreement with previous studies in rat CMs (Gambliel et al., 2002; Zhang et al., 2014). Our findings on the protein level validate the mRNA data to some extent, since the amount of RYR2, CamKII, SERCA, PLN and NCX was comparable between groups (Figure 26 and Figure 27). However, no significant changes were observed upon treatment with low DOX concentrations. Phosphorylation of PLN at S16 and Thr17 was about twice as high in the ACT-iPSC-CMs, although it did not reach statistical significance (Figure 27). This might contribute to the faster transient decay time in ACT-iPSC-CMs at basal conditions (Figure 24), since phosphorylated PLN is inactive resulting in a stronger activity of SERCA. Upon treatment with high DOX concentrations, the amount of RYR2 was reduced in ACT-iPSC-CMs to about $50 \%$ and RYR2 is hyperphosphorylated at S2814 (Figure 26). This means that ACT-iPSC-CMs possess less amounts of RYR2, which is more active upon treatment with high DOX concentrations. In Ctrl-iPSC-CMs, on the other hand, no significant changes in RYR2 amount or phosphorylation were found. Both findings may explain the unchanged $\mathrm{Ca}^{2+}$ transient rise time upon high DOX treatment, but not the reduced transient amplitude (Figure 24). Additionally, the significant decrease of $\mathrm{Ca}^{2+}$ transient rise time and increase of amplitude at low DOX concentration do not seem to be based on RYR2 expression or phosphorylation.

High DOX concentrations induced a significant increase in the amount of SERCA in Ctrl-iPSCCMs but not in ACT-iPSC-CMs (Figure 27). The amount of SERCA-regulating PLN was unchanged in both groups. However, a significant hyperphosphorylation of PLN at S16 was observed in both groups induced by high DOX concentrations. ACT-iPSC-CM PLN was hyperphosphorylated at Thr17 upon application of high DOX concentrations. These findings suggest that the reduction of transient decay time induced by high DOX concentrations (Figure 24) is caused by an upregulation of SERCA expression and PLN-S16 hyperphosphorylation in the control group and by hyperphosphorylation of PLN-S16 and PLN-Thr17 in the ACT patient group. These distinct mechanisms may explain why the 
transient decay time decrease is significantly stronger in the control group. The significant decrease of $\mathrm{Ca}^{2+}$ transient decay time upon low DOX concentrations does not seem to be based on SERCA and PLN expression.

The amount of CamKII is not changed by DOX in both groups, which is consistent with previous findings (Sag et al., 2011). However, the hyperphosphorylation of CamKII target sites S2814 of RYR2 and Thr17 of PLN suggests increased activity of CamKII in ACT-iPSC-CMs but not in Ctrl-iPSC-CMs upon application of high concentrations of DOX. CamKII activity has been shown to be regulated by oxidation (Erickson et al., 2008). Importantly, recent studies conclude that CamKII is a target of NADPH oxidase 2 generated ROS (He et al., 2011; Swaminathan et al., 2011; Zhao et al., 2011). It is tempting to conclude that the observed hyperphosphorylation of CamKII targets in ACT patient iPSC-CMs may be connected to a higher activity of NADPH oxidase 2 upon DOX treatment, which in turn may be caused by SNPs in NADPH oxidase subunit encoding genes. However, more validation is necessary to support such a hypothesis. Especially the NADPH oxidase 2 activity needs to be assessed specifically and a causal relation with the SNPs has to be shown. Furthermore, the oxidation status of CamKII should be analyzed in future experiments in the IPSC-CM ACT model.

In conclusion, we found severe disturbances of $\mathrm{Ca}^{2+}$ homeostasis in both Ctrl- and ACT-iPSCCMs upon DOX treatment. The basis of these may be direct interactions of DOX with key proteins or their oxidation by increased amounts of ROS and DOX-induced changes in gene expression. Furthermore, the DOX-induced mechanisms causing disturbances in $\mathrm{Ca}^{2+}$ transients seem to be different between both groups.

\subsubsection{Apoptosis and cell death}

There is wide acceptance that a common result of different discussed ACT pathomechanisms is cell death of CMs by apoptosis (Carvalho et al., 2014; Zhang et al., 2009). Accordingly, high concentrations of DOX induced a significant amount of apoptosis and cell death in both Ctrland ACT-iPSC-CMs (Figure 29). Unexpectedly, about twice as many Ctrl-iPSC-CMs were apoptotic at culture conditions without DOX treatment compared to the ACT-iPSC-CMs. Upon 24 hours of treatment with low concentrations, the relative DOX-induced increase of apoptotic cells was about twice as high in ACT-iPSC-CMs as in Ctrl-iPSC-CMs. After treatment for 72 hours, the relative increase was higher at all tested DOX concentrations in ACT-iPSCCMs, which was significant at $1 \mu \mathrm{M}$ (Figure 29). These data indicate that ACT-iPSC-CMs are more sensitive to DOX treatment with regard to the development of apoptosis. Similar findings were reported by Burridge et al. who showed that ACT patient iPSC-CMs are more prone to DOX-induced apoptosis than control iPSC-CMs (Burridge et al., 2016). The authors show that iPSC-CMs from ACT patients are significantly more apoptotic upon application of $0.1-10 \mu \mathrm{M}$ DOX for 72 hours using the annexin V / PI assay. 
Several distinct DOX-induced pathways have been described that cause both intrinsic and extrinsic apoptosis of CMs. For example, CMs are more prone to Fas/FasL dependent apoptosis upon DOX application (Yamaoka et al., 2000). Also, pro-survival pathways such as NRG1/ErbB are impaired by DOX, leading to increased rates of cell death (Horie et al., 2010). However, iPSC-CMs were cultured with high purity and no further signaling molecules were tested, which would induce extrinsic apoptosis. Hence, the observed DOX-induced apoptosis is most likely caused by intrinsic signaling. For one, transcription factors or coactivators involved in induction or inhibition of apoptosis are dysregulated by DOX such as GATA-4, CARP, NF-kB, NFAT and p300 (Aihara et al., 2000; Aries et al., 2004; Jeyaseelan et al., 1997; Kalivendi et al., 2005; Kim et al., 2007; Wang et al., 2002). Since DOX-induced ROS generation is often implicated in these dysregulations, the observed increase of ROS in iPSC-CMs upon DOX application (Figure 19 and 22) may be a reason for induction of apoptosis (Aihara et al., 2000; Kalivendi et al., 2005; Kim et al., 2007; Wang et al., 2002). Additionally to transcription factors, other important apoptosis-related proteins are also dysregulated by DOX, such as p53, Akt, MAPKs and Bcl-2 family members (Zhang et al., 2009). p53 signaling and subsequent activation of intrinsic apoptosis is also induced by DNA damage which may be directly caused by DOX or indirectly via TOP2 poisoning and oxidation. Furthermore, DOXinduced organelle damage has been reported to result in apoptosis. For example, elevated cytosolic $\mathrm{Ca}^{2+}$ levels cause increased $\mathrm{Ca}^{2+}$ influx into the mitochondria, which in turn may cause an overload and the disruption of the outer membrane. Thereby, cytochrome $c$ and apoptosis inducing factor (AIF) could be released into the cytoplasm, inducing apoptosis (Childs et al., 2002; Deniaud et al., 2008; Kim et al., 2006). The high number of apoptosis pathways that have been linked to ACT shows the complexity of DOX toxicity. Good evidence has been reported for all of the pathways, which were mentioned above. Therefore, they should all be investigated in the IPSC-CM ACT model in future experiments. Thereby, it may be elucidated whether increased levels of ROS, DNA damage or disturbed $\mathrm{Ca}^{2+}$ handling is the main reason for the DOX-induced apoptosis in iPSC-CMs, which was found in our work.

In conclusion, DOX induces apoptosis and cell death dose-dependently in iPSC-CMs. Furthermore, our findings suggest that ACT-iPSC-CMs more prone to DOX-induced apoptosis than Ctrl-iPSC-CMs. However, until now it is unclear, which apoptosis-related pathways are involved in apoptosis of ACT-iPSC-CMs. This has to be answered in the future. It is plausible to conclude that the DOX-induced ROS generation and dysregulation of $\mathrm{Ca}^{2+}$ signaling, which were observed in this work, may contribute to apoptosis in iPSC-CMs.

\subsubsection{Sarcomeric integrity}

Sarcomeric disarray is a feature of ACT, which can be found in heart biopsies. $\alpha$-actinin is part of the Z-discs, structures that make up the borders of one sarcomere (Minotti et al., 2004). 
Because of its distinct localization within the sarcomeric cytoskeleton, immunofluorescence stainings of $\alpha$-actinin can be used to assess the regularity of sarcomeres. As expected, the regularity of sarcomeres in iPSC-CMs was comparable between ACT patients and controls under basal conditions (Figure 30). Upon treatment with high DOX concentrations, a significant reduction of sarcomeric regularity of about $20 \%$ was found in both groups (Figure 30). Importantly, treatment with low DOX concentrations induced a significantly greater decrease of regularity in ACT-iPSC-CMs than in Ctrl-iPSC-CMs (Figure 30). These findings suggest that ACT-iPSC-CMs react significantly more sensitive towards DOX with regard to the sarcomeric cytoskeleton. Our findings are in line with a previous study by Burridge et al. who claim that iPSC-CMs from ACT patients lose sarcomeric organization at lower DOX concentrations compared to iPSC-CMs from healthy donors (Burridge et al., 2016). In our work, $\alpha$-actinin regularity was quantified with minimal bias by the experimenter using the FFT algorithm and radial integration of the frequency domain, whereas Burridge et al. assessed the sarcomeric organization only visually.

The loss of sarcomeric integrity in our iPSC-CM ACT model may be a result of several DOXinduced mechanisms. For one, the $\mathrm{Ca}^{2+}$-dependent cysteine protease calpain has been implicated in ACT. Independent studies suggest an increase of calpain activity upon DOX treatment and subsequent degradation of essential sarcomeric proteins such as titin and dystrophin (Campos et al., 2011; Chen et al., 2012; Jang et al., 2004; Lim et al., 2004; Min et al., 2015), although another report claims reduced calpain activity (Wang et al., 2013). Increased calpain activity is also connected to other heart conditions such as ischemia/reperfusion-induced myocardial injury and diabetic cardiomyopathy, which favors the role of calpain in ACT (Khalil et al., 2005; Li et al., 2011; Perrin et al., 2003). Additionally, stress-conditions may increase calpain activity and thereby involve it in CM apoptosis and inflammatory response (Li et al., 2009a, 2009b). Degradation of titin and dystrophin may explain the DOX-induced reduction of sarcomeric regularity in Ctrl- and ACT-iPSC-CMs. This is because the loss of $\alpha$-actinin localization regularity does not seem to be connected to the amount of $\alpha$-actinin. The amount of $\alpha$-actinin is not significantly changed by DOX treatment (Figure 31). Furthermore, previous experiments performed by Andrey Fomin from the research group of Prof. Dr. W. A. Linke from the University Hospital of Münster showed titin degradation in iPSC-CMs from healthy control donors upon treatment with high DOX concentrations (see appendix, Figure 40). Since calpain activity depends on $\mathrm{Ca}^{2+}$ binding (Moldoveanu et al., 2002), the reduction of sarcomeric integrity in iPSC-CMs may be caused by DOX-induced changes in calcium homeostasis. As discussed in chapter 5.2.3, we found significant DOX-induced changes of $\mathrm{Ca}^{2+}$ transient properties in both groups. However, the experiments performed in this work are not suitable to assess quantitative amounts of diastolic cytosolic $\mathrm{Ca}^{2+}$, making comparisons between groups impossible. In the future, the 
diastolic cytosolic $\mathrm{Ca}^{2+}$ amount should be determined to analyze a possible connection between DOX-induced dysregulation of $\mathrm{Ca}^{2+}$ handling and sarcomeric integrity.

Interestingly, several reports suggest that calpain activity is positively regulated by NADPH oxidase 2 in CMs and other cell types such as mesangial cells and neurons (Guemez-Gamboa et al., 2011; Li et al., 2009a, 2009c, 2009b; Sheu et al., 2017). Inactivation of NADPH oxidase 2 by depletion of RAC1, on the other hand, results in inhibition of calpain activity in CMs (Shan et al., 2010). In the context of these reports the significantly stronger disturbance of sarcomeric integrity in ACT-iPSC-CMs upon application of low DOX concentrations may be explained by a higher activity of NADPH oxidase 2. The result would be a higher amount of ROS and thereby an increased calpain induced degradation of titin and dystrophin. The reason for this may be the alleles of SNPs in RAC2 and CYBA contained by ACT patients but not controls. However, as mentioned in the previous chapters, such a hypothesis needs further validation, especially regarding the influence of SNPS on NADPH oxidase activity and calpain activity status. Also, titin degradation may be analyzed directly on the protein level by gel electrophoresis, although the size of about 3,6 MDa makes the methodology more complex. First trials were already performed by Andrey Fomin (see appendix, Figure 40).

Another explanation of DOX-induced reduction of sarcomeric integrity in iPSC-CMs may be altered expression of genes encoding for sarcomeric proteins. We found a DOX-induced reduction of expression of $\alpha$-actinin, $\alpha-M H C, \beta-M H C$ and $c T N T$ in iPSC-CMs of both groups. Respective changes on the protein level may contribute to the disturbance of sarcomere regularity upon DOX application. However, since $\alpha$-actinin is not significantly influenced by DOX on the protein level, the amount of $\alpha-M H C, \beta-M H C$ and cTNT needs to be assessed in DOX-treated iPSC-CMs in future experiments. Our findings are supported by reports of DOXinduced decrease of cardiac sarcomeric gene expression on the mRNA level in vitro and in vivo in mice (Chen et al., 2012; Ito et al., 1990). Since no significant differences between ACT patients and controls were found in our work, it is unlikely that altered cardiac gene expression on the mRNA level contributes to the increased sensitivity of ACT patients regarding DOX-induced disruption of sarcomeric integrity.

\subsubsection{Mechanical functionality}

ACT is characterized by decreased heart function, which is often accompanied by reduced LVEF (Minotti et al., 2004; Nousiainen et al., 2002). This reduction of heart function is the result of accumulating damage on the subcellular level. In this work we generated an iPSCCM model of ACT and analyzed underlying damage-causing pathomechanisms such as increase of ROS production and involvement of NADPH oxidases, impairment of $\mathrm{Ca}^{2+}$ handling, induction of apoptosis and reduction of sarcomeric integrity. To determine the ultimate effects of these pathomechanisms, we aimed to assess the overall mechanical 
functionality of Ctrl- and ACT-iPSC-CMs upon DOX application. For this purpose, analyses of the mechanical beating behavior of iPSC-CMs in vitro were necessary. However, these are challenging and often require advanced equipment and training. As a result, few studies describing iPSC-CM disease models assess the mechanical functionality but rather rely on electrophysiological analyses (Laurila et al., 2016). The force of contraction of a single iPSCCM may be determined by traction force microscopy in which iPSC-CMs are cultured on a substrate containing fluorescent beats. By observing the displacement of beats, which is caused by beating iPSC-CMs, the force of contraction can be calculated (Wang and Lin, 2007). Furthermore, atomic force microscopy may be used to analyze beating characteristics of iPSC-CMs. By applying a small cantilever to the cell surface, the CM contraction can be observed (Domke et al., 1999). Impedance assays and video microscopy are additional computational approaches to determine iPSC-CM beating characteristics (Laurila et al., 2016; Peters et al., 2015). Unfortunately, approaches that assess single iPSC-CMs or monolayers may not reproduce phenotypes that develop in a tissue context.

3D-cultures such as EHMs offer the opportunity to analyze the functionality of iPSC-CMs in a model of the human myocardium. This state-of-the-art approach furthermore facilitates iPSC-CM maturation as indicated by structural and functional properties (Tiburcy et al., 2017). In our work, we generated EHMs from iPSC-CM of ACT patients and controls. EHMs from both groups showed an increase in beating frequency and irregular beating upon DOX application. These findings suggest that EHMs generated from iPSC-CMs recapitulate DOXinduced symptoms of ACT and are therefore a suitable tool to model functional phenotypes of ACT (Bristow et al., 1978; Ewer et al., 2003; Octavia et al., 2012; Rudzinski et al., 2007). Interestingly, the increase of beating frequency of ACT-EHMs was significantly greater compared to Ctrl-EHMs, suggesting a higher susceptibility to DOX (Figure 32). The DOXinduced changes in beating properties of EHMs likely result from the observed impairment of $\mathrm{Ca}^{2+}$ handling (Figure 24). The decrease of $\mathrm{Ca}^{2+}$ transient rise time and decay time, which was observed in iPSC-CMs upon DOX treatment, favors fastening of beating but also arrhythmia. However, our findings regarding $\mathrm{Ca}^{2+}$ handling do not explain, why the DOX-induced increase of beating frequency is higher in the ACT-EHMs, because changes in $\mathrm{Ca}^{2+}$ transient properties were greater in Ctrl-iPSC-CMs.

The most important finding of this work was that DOX application caused a significant reduction of force of contraction of ACT-EHMs but not of Ctrl-EHMs (Figure 33). This effect was even stronger, when the force of contraction was normalized to the CSA (Figure 33). To rule out that the differences in DOX-dependent force of contraction between Ctrl- and ACTEHMs are based on different CM content in the EHMs, immunhistochemical stainings of EHM should be performed in the future. In this way, it would be possible to analyze the CMs and the percentage of matrix in each EHM ring. Our data suggests that iPSC-CMs recapitulate ACT 
patient-specific impairments on a functional level, highlighting the importance of the ACT model generated in this work. The DOX-induced reduction of contraction force in ACT-EHMs but not Ctrl-EHMs is likely a result of impaired sarcomeric integrity and increased apoptosis of iPSC-CMs. Both parameters were shown to be affected stronger in ACT-iPSC-CMs upon treatment with clinically relevant DOX concentrations compared to Ctrl-iPSC-CMs (Figure 29 and Figure 30). The sarcomeric integrity governs the generated force of an iPSC-CM, whereas the number of vital iPSC-CMs influence the maximal force generated by EHMs. DOX-induced changes in $\mathrm{Ca}^{2+}$ handling may also contribute to reduction of EHM force. For example, increased diastolic $\mathrm{Ca}^{2+}$ concentrations would cause an incomplete relaxation of sarcomeres and thereby a reduction of force. However, the parameters of $\mathrm{Ca}^{2+}$ handling observed in this work are not sufficient to determine the influence on EHM maximal force. More work regarding $\mathrm{SR} \mathrm{Ca}{ }^{2+}$ content and diastolic $\mathrm{Ca}^{2+}$ concentrations have to be performed in the future. To our knowledge, this is the first report of ACT patient-specific iPSC-CMs recapitulating DOX-induced phenotypes regarding the contractile force.

\subsubsection{Screening for protectants against ACT using the iPSC-CM model}

A main advantage of disease modeling using iPSC-CMs is the possibility to screen for drugs. In this work, first trials were performed to use the iPSC-CM ACT model for screening of cardioprotectants against ACT, which may be used during chemotherapy. In clinical trials, DEX had cardioprotective effects during chemotherapy and is approved for adult patients who already received over $300 \mathrm{mg} / \mathrm{m}^{2}$ DOX (FDA, 2011). Since iron chelating by DEX and subsequent attenuation of DOX-induced oxidative stress may be a way of action, we analyzed the effects of DEX on the production of $\mathrm{H}_{2} \mathrm{O}_{2}$ in iPSC-CMs using the Amplex Red assay (Figure 19). Application of $100 \mu \mathrm{M}$ DEX did not alter $\mathrm{H}_{2} \mathrm{O}_{2}$ production in untreated ACT-iPSC-CMs or upon treatment with $0.5 \mu \mathrm{M}$ DOX. A mild decrease of $\mathrm{H}_{2} \mathrm{O}_{2}$ production was observed in CtrliPSC-CMs. This is in good agreement with the hypothesis that DEX cardioprotection is not a result of iron chelating or reduction of ROS but is rather caused by TOP2 inhibition (Martin et al., 2009). Hence, the effects of DEX on parameters such as DOX-induced apoptosis should further be investigated in the IPSC-CM ACT model. Interestingly, it was reported that DEX application aggravates DOX-induced reduction of iPSC-CM viability (Burridge et al., 2016). However, the authors treated the iPSC-CMs with 100 mM DEX, which is considerably higher than the clinically relevant concentration of about $100 \mu \mathrm{M}$, which was used in our work (Deng et al., 2015; Hochster et al., 1992; Sparano et al., 1999).

The selective late sodium current blocker RAN was the second drug that was tested in this work for its protective effects against DOX. Recently, accumulating evidence shows that RAN attenuates DOX-induced symptoms such as diastolic dysfunction and increased mortality, 
which is accompanied by reduced oxidative stress (Cappetta et al., 2017; Maurea et al., 2016; Tocchetti et al., 2014). In our work, first experiments of RAN-treated iPSC-CMs resulted in reduction of late sodium current as proof of principle (data not shown). But, RAN application did not alter DOX-induced $\mathrm{H}_{2} \mathrm{O}_{2}$ production in ACT-iPSC-CMs (Figure 19). Surprisingly, RAN application significantly increased $\mathrm{H}_{2} \mathrm{O}_{2}$ production in DOX-treated Ctrl-iPSC-CMs. Since sodium currents are strongly connected to $\mathrm{Ca}^{2+}$ handling in $\mathrm{CMs}$, these findings may be related to the differences in $\mathrm{Ca}^{2+}$ handling properties between Ctrl- and ACT-iPSC-CMs. Therefore, it will be interesting to analyze the effects of RAN on $\mathrm{Ca}^{2+}$ transient in the iPSC-CM ACT-model.

Due to limited time, we could not expand the analyses of cardioprotectants in this work. However, application of additional DEX and RAN concentrations may reveal further effects. Similarly, the variation of application time seems promising. Several candidates for prophylactic treatment against ACT have been reported, among those are plant-derived small molecules, metallothionein, visnagin, diphenylurea and resveratrol (Gu et al., 2015; Kimura et al., 2000; Liu et al., 2014; Ojha et al., 2016).

\subsubsection{Limitations of this work}

The present work was limited by several factors. As discussed above (see chapter 5.1.2), iPSC-CMs generated with current protocols do not resemble adult human CMs. Hence, it is possible that ACT patient-specific phenotypes that develop in the context of an adult CM are not present in our iPSC-CM model. Accordingly, the phenotypes that were identified in our work might not develop in more mature CMs. While it is beneficial to model a disease in a single cell type, the pathophysiology of the heart is influenced by other cell types despite CMs. In our model, the effect of additional cell types, such as cardiac fibroblasts, cells of the immune system or endothelial cells is not represented.

The hiPSC technology offers great advantages over animal models. On the other hand, several disadvantages are implied. Animal models are usually breed to achieve isogenicity. Thereby, the effects of the genetic background are reduced in animal disease modeling. The genetic variability between human individuals is much greater. Therefore, it is possible that phenotypes that were identified in our iPSC-CM model are influenced by differences in the genetic background. An additional disadvantage of the hiPSC model is the high effort, which is needed to recruit an individual donor and generate and maintain iPSCs. As a result, studies of hiPSC-based disease models are often performed with considerably fewer biological samples than studies with animal models. Accordingly, the sample number of our study was small with 2 controls and 3 ACT patients. 
The definition of ACT is not consistent between studies. The recruitment of actual ACT patients is therefore complicated. Similarly, recruitment of controls is hampered by the fact that ACT can develop years after chemotherapy. As a result, it is possible that ACT patients in this work did not actually develop ACT and that controls were really ACT patients. This is because heart conditions that were diagnosed in ACT patients during chemotherapy may also have been unrelated to DOX and rather have been caused by high age, for example. Controls could have developed chemotherapy-induced heart conditions after they were recruited for our work. Furthermore, milder ACT-associated symptoms may not have been detected in a chemotherapy patient. Subsequently, the patient would have been falsely classified as control.

\subsubsection{Conclusions and proposed model}

Figure 34 summarizes the findings of this work. We show that ACT-iPSC-CMs recapitulate the DOX-induced reduction of mechanical functionality. We postulate that DOX-induced impairment of sarcomeric integrity and increase of apoptosis are the basis of attenuated contractile force in ACT-iPSC-CMs. Furthermore, Ctrl- and ACT-iPSC-CMs showed disturbances in $\mathrm{Ca}^{2+}$ handling upon DOX application, which may be caused by distinct pathways. These findings likely explain the DOX-induced increase in beating frequency and irregular beating, which was observed in both groups. ACT-iPSC-CMs showed a higher production of ROS at basal conditions compared to Ctrl-iPSC-CMs. Furthermore, the amount of ROS was increased in both groups upon DOX application, which likely influences aforementioned parameters. Unfortunately, we could not determine the direct influence of NADPH oxidases or ACT-associated SNPs in the established iPSC-CM ACT model. 


\section{Control}

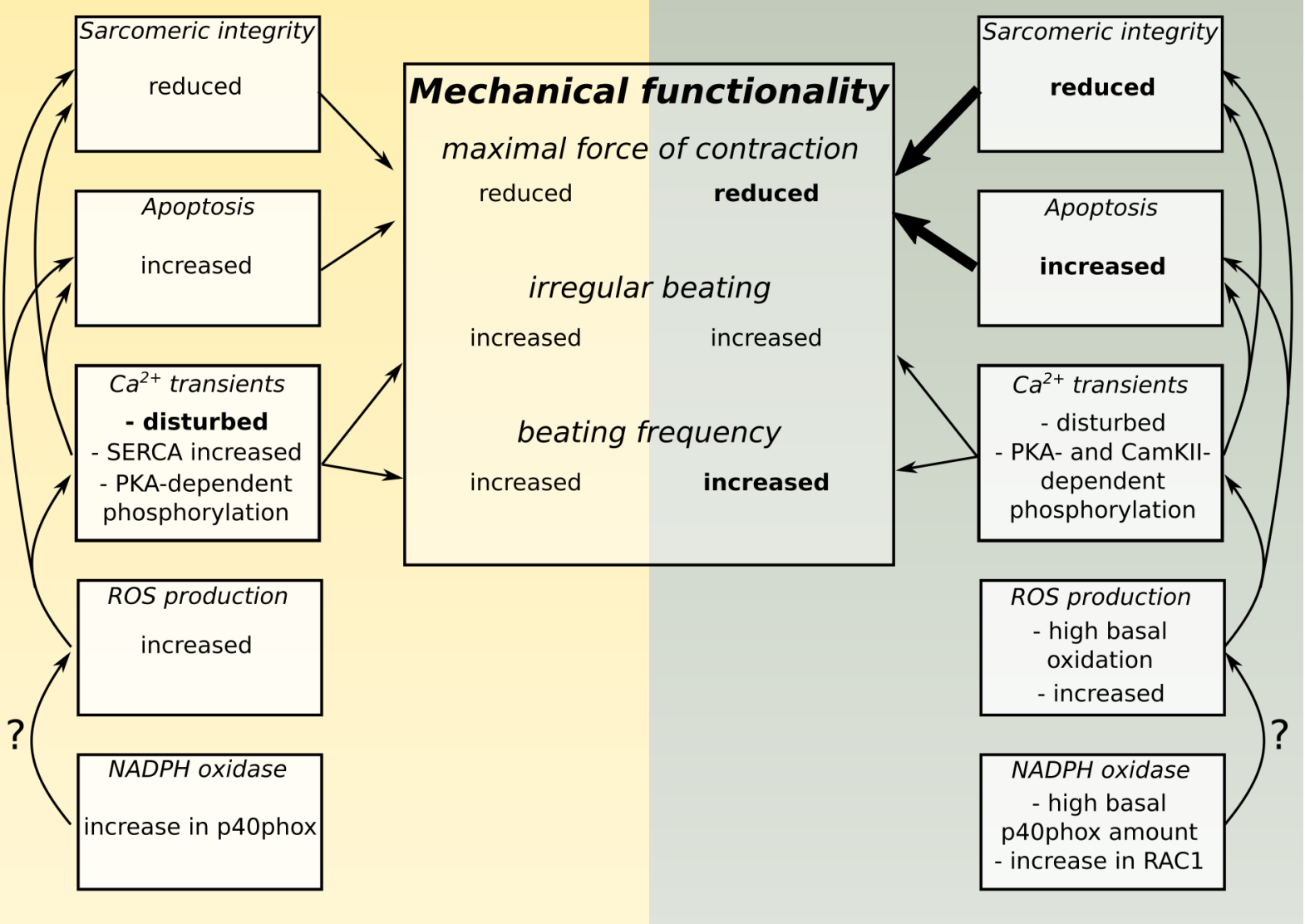

\section{ACT patient}

Figure 34: Schematic summary of the iPSC-CM ACT model. The boxes depict the parameters that were analyzed in this work in Ctrl-iPSC-CMs (yellow background) and ACT-iPSC-CMs (green background). The described changes refer to DOX-induced changes. Bold text represents stronger effects in the respective group. Arrows indicate proposed connections between findings, based on current literature. Bold arrows represent a strong influence.

\subsubsection{Outlook}

In this work, an iPSC-CM based model of ACT was established and the focus was mainly on the analysis of phenotypes that occurred after a single DOX application for 24 hours with varying concentrations. Thereby, we found 0.25 and $5 \mu \mathrm{M}$ DOX to be concentrations that induce changes in iPSC-CM functionality and may reflect steady-state and peak concentrations in the serum of patients during chemotherapy. In the future, it would be interesting to investigate these phenotypes with different time intervals between single DOX applications and analyses. The aim of such an experimental design would be to determine whether DOX-induced phenotypes in iPSC-CMs attenuate, aggravate or remain unchanged over time. Thereby, a possible reversibility of ACT could be investigated. In this work, first analyses of ROS production using such an experimental design suggest recovery to a certain degree (Figure 19). However, increased ROS production was still observed 21 days after single DOX treatment, which indicates chronic, non-reversible alterations. In this work, the 
DOX-induced disruption of sarcomeric integrity is suggested to be a main cause for the reduction of maximal force of EHMs. Therefore, it would be especially interesting to observe the dynamics of sarcomeric integrity after single DOX application. Additionally to the extension of follow-up time after DOX application, the effects of cumulative DOX treatment should also be investigated. Thereby, application schedules during chemotherapy may be mimicked more closely and phenotypes may be observed that result from accumulating damage.

We used the iPSC-CM ACT model to test the cardioprotective effects of DEX and RAN on the production of ROS upon DOX application. In the future, the screening for protectants against ACT should be expanded. As discussed in chapter 5.2.7, plant-derived small molecules, metallothionein, visnagin, diphenylurea and resveratrol are promising candidates. It will be interesting to analyze cardioprotective effects of drugs on additional ACT pathomechanisms investigated in this work. Especially analyses of DOX-induced apoptosis and disruption of sarcomeric integrity seem promising, since they are suggested to be the cause of reduced force of contraction of EHMs.

Unfortunately, the activity of NADPH oxidases and the functional influence of ACT-associated SNPs in RAC2 and CYBA could not be determined in this work. In the future, assessing the activity of NADPH oxidases in living iPSC-CMs will be crucial to assess the influence of SNPS for the development of ACT. Recently developed gene editing technology such as CRISPR/Cas9 should be applied in Ctrl- and ACT-iPSCs (Chaterji et al., 2017). In this way, iPSC cell lines can be generated that only differ in the allele of the respective SNP and are otherwise isogenic. Such isogenic iPSCs would be a powerful means to analyze functional consequences of ACT-associated SPNs in human CMs. Furthermore, recently described inhibitors, which are more specific against NADPH oxidases, may help to determine the source of observed ROS production. It will also be interesting to determine the effects of such inhibitors on the remaining pathomechanisms of ACT investigated in this work, to reveal the influence of NADPH oxidase based redox cycling. 


\section{References}

Abramson, J.J., Buck, E., Salama, G., Casida, J.E., and Pessah, I.N. (1988). Mechanism of anthraquinone-induced calcium release from skeletal muscle sarcoplasmic reticulum. J. Biol. Chem. 263, 18750-18758.

Ai, X., Curran, J.W., Shannon, T.R., Bers, D.M., and Pogwizd, S.M. (2005).

$\mathrm{Ca} 2+/$ calmodulin-dependent protein kinase modulates cardiac ryanodine receptor

phosphorylation and sarcoplasmic reticulum Ca2+ leak in heart failure. Circ. Res. 97, 13141322.

Aihara, Y., Kurabayashi, M., Tanaka, T., Takeda, S.I., Tomaru, K., Sekiguchi, K.I., Ohyama, Y., and Nagai, R. (2000). Doxorubicin represses CARP gene transcription through the generation of oxidative stress in neonatal rat cardiac myocytes: possible role of serine/threonine kinase-dependent pathways. J. Mol. Cell. Cardiol. 32, 1401-1414.

Airoldi, M., Amadori, D., Barni, S., Cinieri, S., De Placido, S., Di Leo, A., Gennari, A., Iacobelli, S., Ionta, M.T., Lorusso, V., et al. (2011). Clinical activity and cardiac tolerability of non-pegylated liposomal doxorubicin in breast cancer: a synthetic review. Tumori 97, 690692.

Altenhöfer, S., Radermacher, K.A., Kleikers, P.W.M., Wingler, K., and Schmidt, H.H.H.W. (2015). Evolution of NADPH Oxidase Inhibitors: Selectivity and Mechanisms for Target Engagement. Antioxid. Redox Signal. 23, 406-427.

Anderson, C.W., Boardman, N., Luo, J., Park, J., and Qyang, Y. (2017). Stem Cells in Cardiovascular Medicine: the Road to Regenerative Therapies. Curr. Cardiol. Rep. 19, 34.

Angsutararux, P., Luanpitpong, S., and Issaragrisil, S. (2015). Chemotherapy-Induced Cardiotoxicity: Overview of the Roles of Oxidative Stress. Oxid. Med. Cell. Longev. 2015.

Anilkumar, N., San Jose, G., Sawyer, I., Santos, C.X.C., Sand, C., Brewer, A.C., Warren, D., and Shah, A.M. (2013). A 28-kDa splice variant of NADPH oxidase-4 is nuclear-localized and involved in redox signaling in vascular cells. Arterioscler. Thromb. Vasc. Biol. 33, e104-112.

Aoi, T. (2016). 10th anniversary of iPS cells: the challenges that lie ahead. J. Biochem. (Tokyo) 160, 121-129.

Arcamone, F., Cassinelli, G., Fantini, G., Grein, A., Orezzi, P., Pol, C., and Spalla, C. (1969). Adriamycin, 14-hydroxydaunomycin, a new antitumor antibiotic from S. peucetius var. caesius. Biotechnol. Bioeng. 11, 1101-1110.

Aries, A., Paradis, P., Lefebvre, C., Schwartz, R.J., and Nemer, M. (2004). Essential role of GATA-4 in cell survival and drug-induced cardiotoxicity. Proc. Natl. Acad. Sci. U. S. A. 101, 6975-6980.

Beers, J., Linask, K.L., Chen, J.A., Siniscalchi, L.I., Lin, Y., Zheng, W., Rao, M., and Chen, G. (2015). A cost-effective and efficient reprogramming platform for large-scale production of 
integration-free human induced pluripotent stem cells in chemically defined culture. Sci. Rep. 5, srep11319.

Bellin, M., Marchetto, M.C., Gage, F.H., and Mummery, C.L. (2012). Induced pluripotent stem cells: the new patient? Nat. Rev. Mol. Cell Biol. 13, 713-726.

Belmonte, F., Das, S., Sysa-Shah, P., Sivakumaran, V., Stanley, B., Guo, X., Paolocci, N., Aon, M.A., Nagane, M., Kuppusamy, P., et al. (2015). ErbB2 overexpression upregulates antioxidant enzymes, reduces basal levels of reactive oxygen species, and protects against doxorubicin cardiotoxicity. Am. J. Physiol. - Heart Circ. Physiol. 309, H1271-H1280.

Belousov, V.V., Fradkov, A.F., Lukyanov, K.A., Staroverov, D.B., Shakhbazov, K.S., Terskikh, A.V., and Lukyanov, S. (2006). Genetically encoded fluorescent indicator for intracellular hydrogen peroxide. Nat. Methods 3, 281-286.

Benjamin, R.S., Riggs, C.E., and Bachur, N.R. (1977). Plasma Pharmacokinetics of Adriamycin and Its Metabolites in Humans with Normal Hepatic and Renal Function. Cancer Res. 37, 1416-1420.

Bers, D.M. (2004). Macromolecular complexes regulating cardiac ryanodine receptor function. J. Mol. Cell. Cardiol. 37, 417-429.

Bers, D.M., Barry, W.H., and Despa, S. (2003). Intracellular Na+ regulation in cardiac myocytes. Cardiovasc. Res. 57, 897-912.

Berthiaume, J.M., and Wallace, K.B. (2007). Adriamycin-induced oxidative mitochondrial cardiotoxicity. Cell Biol. Toxicol. 23, 15-25.

Berthiaume, J.M., Oliveira, P.J., Fariss, M.W., and Wallace, K.B. (2005). Dietary vitamin E decreases doxorubicin-induced oxidative stress without preventing mitochondrial dysfunction. Cardiovasc. Toxicol. 5, 257-267.

Bian, Y., Sun, M., Silver, M., Ho, K.K.L., Marchionni, M.A., Caggiano, A.O., Stone, J.R., Amende, I., Hampton, T.G., Morgan, J.P., et al. (2009). Neuregulin-1 attenuated doxorubicininduced decrease in cardiac troponins. Am. J. Physiol. - Heart Circ. Physiol. 297, H1974H1983.

Biberstine-Kinkade, K.J., DeLeo, F.R., Epstein, R.I., LeRoy, B.A., Nauseef, W.M., and Dinauer, M.C. (2001). Heme-ligating Histidines in Flavocytochromeb 558 identification of specific histidines in gp91 phox. J. Biol. Chem. 276, 31105-31112.

Biberstine-Kinkade, K.J., Yu, L., Stull, N., LeRoy, B., Bennett, S., Cross, A., and Dinauer, M.C. (2002). Mutagenesis of p22 phox Histidine 94 a histidine in this position is not required for flovocytochrome b 558 function. J. Biol. Chem. 277, 30368-30374.

Bilan, D.S., and Belousov, V.V. (2017). New tools for redox biology: From imaging to manipulation. Free Radic. Biol. Med. 109, 167-188.

Bilan, D.S., Pase, L., Joosen, L., Gorokhovatsky, A.Y., Ermakova, Y.G., Gadella, T.W.J., Grabher, C., Schultz, C., Lukyanov, S., and Belousov, V.V. (2013). HyPer-3: A Genetically 
Encoded H2O2 Probe with Improved Performance for Ratiometric and Fluorescence Lifetime Imaging. ACS Chem. Biol. 8, 535-542.

Blaes, A., Duprez, D., Defor, T., Shanley, R., Beckwith, H., Haddad, T., Potter, D., Yee, D., Sanghavi, K., and Jacobson, P. (2015). Angiotensin Converting Enzyme Inhibitors (ACEI) and doxorubicin pharmacokinetics in women receiving adjuvant breast cancer treatment. SpringerPlus 4.

Blazeski, A., Zhu, R., Hunter, D.W., Weinberg, S.H., Boheler, K.R., Zambidis, E.T., and Tung, L. (2012). Electrophysiological and contractile function of cardiomyocytes derived from human embryonic stem cells. Prog. Biophys. Mol. Biol. 110, 178-195.

Block, K., Gorin, Y., and Abboud, H.E. (2009). Subcellular localization of Nox4 and regulation in diabetes. Proc. Natl. Acad. Sci. U. S. A. 106, 14385-14390.

Bonini, M.G., Rota, C., Tomasi, A., and Mason, R.P. (2006). The oxidation of 2',7'dichlorofluorescin to reactive oxygen species: a self-fulfilling prophesy? Free Radic. Biol. Med. 40, 968-975.

Borchert, T., Hübscher, D., Guessoum, C.I., Lam, T.-D.D., Ghadri, J.R., Schellinger, I.N., Tiburcy, M., Liaw, N.Y., Li, Y., Haas, J., et al. (2017). Catecholamine-Dependent $\beta$ Adrenergic Signaling in a Pluripotent Stem Cell Model of Takotsubo Cardiomyopathy. J. Am. Coll. Cardiol. 70, 975-991.

Bouin, A.P., Grandvaux, N., Vignais, P.V., and Fuchs, A. (1998). p40(phox) is phosphorylated on threonine 154 and serine 315 during activation of the phagocyte NADPH oxidase. Implication of a protein kinase c-type kinase in the phosphorylation process. J. Biol. Chem. 273, 30097-30103.

Boyer, L.A., Lee, T.I., Cole, M.F., Johnstone, S.E., Levine, S.S., Zucker, J.P., Guenther, M.G., Kumar, R.M., Murray, H.L., Jenner, R.G., et al. (2005). Core transcriptional regulatory circuitry in human embryonic stem cells. Cell 122, 947-956.

Brach, T., Soyk, S., Müller, C., Hinz, G., Hell, R., Brandizzi, F., and Meyer, A.J. (2009). Noninvasive topology analysis of membrane proteins in the secretory pathway. Plant J. 57, 534541.

Brambrink, T., Foreman, R., Welstead, G.G., Lengner, C.J., Wernig, M., Suh, H., and Jaenisch, R. (2008). Sequential expression of pluripotency markers during direct reprogramming of mouse somatic cells. Cell Stem Cell 2, 151-159.

Brandes, R.P., Weissmann, N., and Schröder, K. (2014). Nox family NADPH oxidases: Molecular mechanisms of activation. Free Radic. Biol. Med. 76, 208-226.

Briggs, R., and King, T.J. (1952). Transplantation of Living Nuclei From Blastula Cells into Enucleated Frogs’ Eggs. Proc. Natl. Acad. Sci. U. S. A. 38, 455-463.

Brimble, S.N., Sherrer, E.S., Uhl, E.W., Wang, E., Kelly, S., Merrill, A.H., Robins, A.J., and Schulz, T.C. (2007). The cell surface glycosphingolipids SSEA-3 and SSEA-4 are not essential for human ESC pluripotency. Stem Cells Dayt. Ohio 25, 54-62. 
Brinster, R.L. (1974). The effect of cells transferred into the mouse blastocyst on subsequent development. J. Exp. Med. 140, 1049-1056.

Bristow, M.R., Billingham, M.E., Mason, J.W., and Daniels, J.R. (1978). Clinical spectrum of anthracycline antibiotic cardiotoxicity. Cancer Treat. Rep. 62, 873-879.

Brons, I.G.M., Smithers, L.E., Trotter, M.W.B., Rugg-Gunn, P., Sun, B., Chuva de Sousa Lopes, S.M., Howlett, S.K., Clarkson, A., Ahrlund-Richter, L., Pedersen, R.A., et al. (2007). Derivation of pluripotent epiblast stem cells from mammalian embryos. Nature 448, 191-195.

Bruynzeel, A.M.E., Niessen, H.W.M., Bronzwaer, J.G.F., van der Hoeven, J.J.M., Berkhof, J., Bast, A., van der Vijgh, W.J.F., and van Groeningen, C.J. (2007). The effect of monohydroxyethylrutoside on doxorubicin-induced cardiotoxicity in patients treated for metastatic cancer in a phase II study. Br. J. Cancer 97, 1084-1089.

Buganim, Y., Faddah, D.A., Cheng, A.W., Itskovich, E., Markoulaki, S., Ganz, K., Klemm, S.L., van Oudenaarden, A., and Jaenisch, R. (2012). Single-Cell Expression Analyses during Cellular Reprogramming Reveal an Early Stochastic and a Late Hierarchic Phase. Cell 150, 1209-1222.

Buggisch, M., Ateghang, B., Ruhe, C., Strobel, C., Lange, S., Wartenberg, M., and Sauer, H. (2007). Stimulation of ES-cell-derived cardiomyogenesis and neonatal cardiac cell proliferation by reactive oxygen species and NADPH oxidase. J. Cell Sci. 120, 885-894.

Burgoyne, J.R., Mongue-Din, H., Eaton, P., and Shah, A.M. (2012). Redox Signaling in Cardiac Physiology and Pathology. Circ. Res. 111, 1091-1106.

Burridge, P.W., Matsa, E., Shukla, P., Lin, Z.C., Churko, J.M., Ebert, A.D., Lan, F., Diecke, S., Huber, B., Mordwinkin, N.M., et al. (2014). Chemically defined generation of human cardiomyocytes. Nat. Methods 11, 855-860.

Burridge, P.W., Li, Y.F., Matsa, E., Wu, H., Ong, S.-G., Sharma, A., Holmström, A., Chang, A.C., Coronado, M.J., Ebert, A.D., et al. (2016). Human induced pluripotent stem cell-derived cardiomyocytes recapitulate the predilection of breast cancer patients to doxorubicin-induced cardiotoxicity. Nat. Med. 22, 547-556.

Burritt, J.B., Fritel, G.N., Dahan, I., Pick, E., Roos, D., and Jesaitis, A.J. (2000). Epitope identification for human neutrophil flavocytochrome b monoclonals 48 and 449. Eur. J. Haematol. 65, 407-413.

Campos, E.C., O’Connell, J.L., Malvestio, L.M., Romano, M.M.D., Ramos, S.G., Celes, M.R.N., Prado, C.M., Simões, M.V., and Rossi, M.A. (2011). Calpain-mediated dystrophin disruption may be a potential structural culprit behind chronic doxorubicin-induced cardiomyopathy. Eur. J. Pharmacol. 670, 541-553.

Cao, N., Liu, Z., Chen, Z., Wang, J., Chen, T., Zhao, X., Ma, Y., Qin, L., Kang, J., Wei, B., et al. (2012). Ascorbic acid enhances the cardiac differentiation of induced pluripotent stem cells through promoting the proliferation of cardiac progenitor cells. Cell Res. 22, 219-236. 
Cappetta, D., Esposito, G., Piegari, E., Russo, R., Ciuffreda, L.P., Rivellino, A., Berrino, L., Rossi, F., De Angelis, A., and Urbanek, K. (2016). SIRT1 activation attenuates diastolic dysfunction by reducing cardiac fibrosis in a model of anthracycline cardiomyopathy. Int. J. Cardiol. 205, 99-110.

Cappetta, D., Esposito, G., Coppini, R., Piegari, E., Russo, R., Ciuffreda, L.P., Rivellino, A., Santini, L., Rafaniello, C., Scavone, C., et al. (2017). Effects of ranolazine in a model of doxorubicin-induced left ventricle diastolic dysfunction. Br. J. Pharmacol.

Capranico, G., Tinelli, S., Austin, C.A., Fisher, M.L., and Zunino, F. (1992). Different patterns of gene expression of topoisomerase II isoforms in differentiated tissues during murine development. Biochim. Biophys. Acta 1132, 43-48.

Cardinale, D., Colombo, A., Bacchiani, G., Tedeschi, I., Meroni, C.A., Veglia, F., Civelli, M., Lamantia, G., Colombo, N., Curigliano, G., et al. (2015). Early Detection of Anthracycline Cardiotoxicity and Improvement With Heart Failure Therapy Clinical Perspective. Circulation 131, 1981-1988.

Cartwright, P., McLean, C., Sheppard, A., Rivett, D., Jones, K., and Dalton, S. (2005). LIF/STAT3 controls ES cell self-renewal and pluripotency by a Myc-dependent mechanism. Dev. Camb. Engl. 132, 885-896.

Carvajal-Vergara, X., Sevilla, A., D’Souza, S.L., Ang, Y.-S., Schaniel, C., Lee, D.-F., Yang, L., Kaplan, A.D., Adler, E.D., Rozov, R., et al. (2010). Patient-specific induced pluripotent stem cell derived models of LEOPARD syndrome. Nature 465, 808-812.

Carvalho, F.S., Burgeiro, A., Garcia, R., Moreno, A.J., Carvalho, R.A., and Oliveira, P.J. (2014). Doxorubicin-induced cardiotoxicity: from bioenergetic failure and cell death to cardiomyopathy. Med. Res. Rev. 34, 106-135.

Caspi, O., Huber, I., Kehat, I., Habib, M., Arbel, G., Gepstein, A., Yankelson, L., Aronson, D., Beyar, R., and Gepstein, L. (2007). Transplantation of human embryonic stem cell-derived cardiomyocytes improves myocardial performance in infarcted rat hearts. J. Am. Coll. Cardiol. 50, 1884-1893.

Chambers, I., Colby, D., Robertson, M., Nichols, J., Lee, S., Tweedie, S., and Smith, A. (2003). Functional expression cloning of Nanog, a pluripotency sustaining factor in embryonic stem cells. Cell 113, 643-655.

Chaterji, S., Ahn, E.H., and Kim, D.-H. (2017). CRISPR Genome Engineering for Human Pluripotent Stem Cell Research. Theranostics 7, 4445-4469.

Chaudhari, U., Nemade, H., Wagh, V., Gaspar, J.A., Ellis, J.K., Srinivasan, S.P., Spitkovski, D., Nguemo, F., Louisse, J., Bremer, S., et al. (2015). Identification of genomic biomarkers for anthracycline-induced cardiotoxicity in human iPSC-derived cardiomyocytes: an in vitro repeated exposure toxicity approach for safety assessment. Arch. Toxicol. 90, 2763-2777. 
Chaudhari, U., Nemade, H., Gaspar, J.A., Hescheler, J., Hengstler, J.G., and Sachinidis, A. (2016). MicroRNAs as early toxicity signatures of doxorubicin in human-induced pluripotent stem cell-derived cardiomyocytes. Arch. Toxicol. 90, 3087-3098.

Chaudhari, U., Ellis, J.K., Wagh, V., Nemade, H., Hescheler, J., Keun, H.C., and Sachinidis, A. (2017). Metabolite signatures of doxorubicin induced toxicity in human induced pluripotent stem cell-derived cardiomyocytes. Amino Acids 1-9.

Chen (2012). Topoisomerase II $\alpha$, rather than II $\beta$, is a promising target in development of anticancer drugs. Drug Discov. Ther.

Chen, B., Zhong, L., Roush, S.F., Pentassuglia, L., Peng, X., Samaras, S., Davidson, J.M., Sawyer, D.B., and Lim, C.C. (2012). Disruption of a GATA4/Ankrd1 Signaling Axis in Cardiomyocytes Leads to Sarcomere Disarray: Implications for Anthracycline Cardiomyopathy. PLoS ONE 7.

Chen, G., Gulbranson, D.R., Hou, Z., Bolin, J.M., Ruotti, V., Probasco, M.D., Smuga-Otto, K., Howden, S.E., Diol, N.R., Propson, N.E., et al. (2011). Chemically defined conditions for human iPSC derivation and culture. Nat. Methods 8, 424-429.

Chen, X., Xu, H., Yuan, P., Fang, F., Huss, M., Vega, V.B., Wong, E., Orlov, Y.L., Zhang, W., Jiang, J., et al. (2008). Integration of external signaling pathways with the core transcriptional network in embryonic stem cells. Cell 133, 1106-1117.

Cheung, J.Y., Zhang, X.-Q., Song, J., Gao, E., Chan, T.O., Rabinowitz, J.E., Koch, W.J., Feldman, A.M., and Wang, J. (2013). Coordinated regulation of cardiac $\mathrm{Na}(+) / \mathrm{Ca}(2+)$ exchanger and $\mathrm{Na}(+)-\mathrm{K}(+)$-ATPase by phospholemman (FXYD1). Adv. Exp. Med. Biol. $961,175-190$.

Chihara, D., Westin, J.R., Oki, Y., Ahmed, M.A., Do, B., Fayad, L.E., Hagemeister, F.B., Romaguera, J.E., Fanale, M.A., Lee, H.J., et al. (2016). Management strategies and outcomes for very elderly patients with diffuse large B-cell lymphoma. Cancer 122, 3145-3151.

Childs, A.C., Phaneuf, S.L., Dirks, A.J., Phillips, T., and Leeuwenburgh, C. (2002). Doxorubicin treatment in vivo causes cytochrome $\mathrm{C}$ release and cardiomyocyte apoptosis, as well as increased mitochondrial efficiency, superoxide dismutase activity, and Bcl-2:Bax ratio. Cancer Res. 62, 4592-4598.

Chong, J.J.H., Yang, X., Don, C.W., Minami, E., Liu, Y.-W., Weyers, J.J., Mahoney, W.M., Van Biber, B., Cook, S.M., Palpant, N.J., et al. (2014). Human embryonic-stem-cell-derived cardiomyocytes regenerate non-human primate hearts. Nature 510, 273-277.

Choudhary, P., and Whiting, P.J. (2016). A strategy to ensure safety of stem cell-derived retinal pigment epithelium cells. Stem Cell Res. Ther. 7, 127.

Crone, S.A., Zhao, Y.-Y., Fan, L., Gu, Y., Minamisawa, S., Liu, Y., Peterson, K.L., Chen, J., Kahn, R., Condorelli, G., et al. (2002). ErbB2 is essential in the prevention of dilated cardiomyopathy. Nat. Med. 8, 459-465. 
Cusack, B.J., Mushlin, P.S., Voulelis, L.D., Li, X., Boucek, R.J., and Olson, R.D. (1993). Daunorubicin-induced cardiac injury in the rabbit: a role for daunorubicinol? Toxicol. Appl. Pharmacol. 118, 177-185.

van Dalen, E.C., Caron, H.N., Dickinson, H.O., and Kremer, L.C.M. (2008). Cardioprotective interventions for cancer patients receiving anthracyclines. Cochrane Database Syst. Rev.

van Dalen, E.C., van der Pal, H.J.H., Caron, H.N., and Kremer, L.C. (2009). Different dosage schedules for reducing cardiotoxicity in cancer patients receiving anthracycline chemotherapy. Cochrane Database Syst. Rev.

van Dalen, E.C., Michiels, E.M., Caron, H.N., and Kremer, L.C. (2010). Different anthracycline derivates for reducing cardiotoxicity in cancer patients. Cochrane Database Syst. Rev.

Davies, K.J., and Doroshow, J.H. (1986). Redox cycling of anthracyclines by cardiac mitochondria. I. Anthracycline radical formation by NADH dehydrogenase. J. Biol. Chem. 261, 3060-3067.

De Angelis, A., Piegari, E., Cappetta, D., Marino, L., Filippelli, A., Berrino, L., FerreiraMartins, J., Zheng, H., Hosoda, T., Rota, M., et al. (2010). Anthracycline cardiomyopathy is mediated by depletion of the cardiac stem cell pool and is rescued by restoration of progenitor cell function. Circulation 121, 276-292.

Delaunay, A., Pflieger, D., Barrault, M.B., Vinh, J., and Toledano, M.B. (2002). A thiol peroxidase is an $\mathrm{H} 2 \mathrm{O} 2$ receptor and redox-transducer in gene activation. Cell 111, 471-481.

Deng, S., Yan, T., Nikolova, T., Fuhrmann, D., Nemecek, A., Gödtel-Armbrust, U., Kaina, B., and Wojnowski, L. (2015). The catalytic topoisomerase II inhibitor dexrazoxane induces DNA breaks, ATF3 and the DNA damage response in cancer cells. Br. J. Pharmacol. 172, 22462257.

Deniaud, A., Sharaf el dein, O., Maillier, E., Poncet, D., Kroemer, G., Lemaire, C., and Brenner, C. (2008). Endoplasmic reticulum stress induces calcium-dependent permeability transition, mitochondrial outer membrane permeabilization and apoptosis. Oncogene 27, 285299.

Devalla, H.D., Schwach, V., Ford, J.W., Milnes, J.T., El-Haou, S., Jackson, C., Gkatzis, K., Elliott, D.A., Chuva de Sousa Lopes, S.M., Mummery, C.L., et al. (2015). Atrial-like cardiomyocytes from human pluripotent stem cells are a robust preclinical model for assessing atrial-selective pharmacology. EMBO Mol. Med. 7, 394-410.

Di Marco, A., Cassinelli, G., and Arcamone, F. (1981). The discovery of daunorubicin. Cancer Treat. Rep. 65 Suppl 4, 3-8.

Dikalov, S., Griendling, K.K., and Harrison, D.G. (2007). Measurement of Reactive Oxygen Species in Cardiovascular Studies. Hypertension 49, 717-727. 
Domke, J., Parak, W.J., George, M., Gaub, H.E., and Radmacher, M. (1999). Mapping the mechanical pulse of single cardiomyocytes with the atomic force microscope. Eur. Biophys. J. EBJ 28, 179-186.

Dooley, C.T., Dore, T.M., Hanson, G.T., Jackson, W.C., Remington, S.J., and Tsien, R.Y. (2004). Imaging Dynamic Redox Changes in Mammalian Cells with Green Fluorescent Protein Indicators. J. Biol. Chem. 279, 22284-22293.

Doroshow, J.H. (1983). Effect of anthracycline antibiotics on oxygen radical formation in rat heart. Cancer Res. 43, 460-472.

Doroshow, J.H., Locker, G.Y., and Myers, C.E. (1980). Enzymatic defenses of the mouse heart against reactive oxygen metabolites: alterations produced by doxorubicin. J. Clin. Invest. $65,128-135$.

Doroshow, J.H., Locker, G.Y., Ifrim, I., and Myers, C.E. (1981). Prevention of doxorubicin cardiac toxicity in the mouse by N-acetylcysteine. J. Clin. Invest. 68, 1053-1064.

Dresdale, A.R., Barr, L.H., Bonow, R.O., Mathisen, D.J., Myers, C.E., Schwartz, D.E., d'Angelo, T., and Rosenberg, S.A. (1982). Prospective randomized study of the role of Nacetyl cysteine in reversing doxorubicin-induced cardiomyopathy. Am. J. Clin. Oncol. 5, 657663.

Dubois, N.C., Craft, A.M., Sharma, P., Elliott, D.A., Stanley, E.G., Elefanty, A.G., Gramolini, A., and Keller, G. (2011). SIRPA is a specific cell-surface marker for isolating cardiomyocytes derived from human pluripotent stem cells. Nat. Biotechnol. 29, 1011.

Eisner, D.A., Caldwell, J.L., Kistamás, K., and Trafford, A.W. (2017). Calcium and Excitation-Contraction Coupling in the Heart. Circ. Res. 121, 181-195.

Eldridge, S., Guo, L., Mussio, J., Furniss, M., Hamre, J., and Davis, M. (2014). Examining the Protective Role of ErbB2 Modulation in Human-Induced Pluripotent Stem Cell-Derived Cardiomyocytes. Toxicol. Sci. 141, 547-559.

Elens, L., Tyteca, D., Panin, N., Courtoy, P., Lison, D., Demoulin, J., and Haufroid, V. (2011). Functional defect caused by the 4544g>a Snp in ABCC2: potential impact for drug cellular disposition. Pharmacogenet. Genomics 21, 884-893.

Erickson, J.R., Joiner, M.A., Guan, X., Kutschke, W., Yang, J., Oddis, C.V., Bartlett, R.K., Lowe, J.S., O’Donnell, S.E., Aykin-Burns, N., et al. (2008). A dynamic pathway for calciumindependent activation of CaMKII by methionine oxidation. Cell 133, 462-474.

Evans, M.J., and Kaufman, M.H. (1981). Establishment in culture of pluripotential cells from mouse embryos. Nature 292, 154-156.

Ewer, M.S., Benjamin, R.S., and Yeh, E.T.H. (2003). Cardiac complications of cancer treatment. 
Fan, L.M., Teng, L., and Li, J.-M. (2009). Knockout of p47phox Uncovers a Critical Role of p40phox in Reactive Oxygen Species Production in Microvascular Endothelial Cells. Arterioscler. Thromb. Vasc. Biol. 29, 1651-1656.

FDA (2011). Drug Safety and Availability - FDA Statement on Dexrazoxane. Https://Www.Fda.Gov/Drugs/DrugSafety/Ucm263729.Htm.

Fu, Y., Huang, C., Xu, X., Gu, H., Ye, Y., Jiang, C., Qiu, Z., and Xie, X. (2015). Direct reprogramming of mouse fibroblasts into cardiomyocytes with chemical cocktails. Cell Res. $25,1013-1024$.

Fusaki, N., Ban, H., Nishiyama, A., Saeki, K., and Hasegawa, M. (2009). Efficient induction of transgene-free human pluripotent stem cells using a vector based on Sendai virus, an RNA virus that does not integrate into the host genome. Proc. Jpn. Acad. Ser. B Phys. Biol. Sci. 85, 348-362.

Gafni, O., Weinberger, L., Mansour, A.A., Manor, Y.S., Chomsky, E., Ben-Yosef, D., Kalma, Y., Viukov, S., Maza, I., Zviran, A., et al. (2013). Derivation of novel human ground state naive pluripotent stem cells. Nature 504, 282-286.

Gambliel, H.A., Burke, B.E., Cusack, B.J., Walsh, G.M., Zhang, Y.L., Mushlin, P.S., and Olson, R.D. (2002). Doxorubicin and C-13 deoxydoxorubicin effects on ryanodine receptor gene expression. Biochem. Biophys. Res. Commun. 291, 433-438.

Gilleron, M., Marechal, X., Montaigne, D., Franczak, J., Neviere, R., and Lancel, S. (2009). NADPH oxidases participate to doxorubicin-induced cardiac myocyte apoptosis. Biochem. Biophys. Res. Commun. 388, 727-731.

Giordano, S.H., Lin, Y.-L., Kuo, Y.F., Hortobagyi, G.N., and Goodwin, J.S. (2012). Decline in the Use of Anthracyclines for Breast Cancer. J. Clin. Oncol. 30, 2232-2239.

Gonzalez, R., Lee, J.W., and Schultz, P.G. (2011). Stepwise Chemically Induced Cardiomyocyte Specification of Human Embryonic Stem Cells. Angew. Chem. Int. Ed. 50, 11181-11185.

Goormaghtigh, E., Huart, P., Praet, M., Brasseur, R., and Ruysschaert, J.M. (1990). Structure of the adriamycin-cardiolipin complex. Role in mitochondrial toxicity. Biophys. Chem. 35, 247-257.

Gosalvez, M., Blanco, M., Hunter, J., Miko, M., and Chance, B. (1974). Effects of anticancer agents on the respiration of isolated mitochondria and tumor cells. Eur. J. Cancer 1965 10, 567-574.

Gratia, S., Kay, L., Michelland, S., Sève, M., Schlattner, U., and Tokarska-Schlattner, M. (2012). Cardiac phosphoproteome reveals cell signaling events involved in doxorubicin cardiotoxicity. J. Proteomics 75, 4705-4716.

Greene, R.F., Collins, J.M., Jenkins, J.F., Speyer, J.L., and Myers, C.E. (1983). Plasma Pharmacokinetics of Adriamycin and Adriamycinol: Implications for the Design of in Vitro Experiments and Treatment Protocols. Cancer Res. 43, 3417-3421. 
Griendling, K.K., Touyz, R.M., Zweier, J.L., Dikalov, S., Chilian, W., Chen, Y.-R., Harrison, D.G., and Bhatnagar, A. (2016). Measurement of Reactive Oxygen Species, Reactive Nitrogen Species, and Redox-Dependent Signaling in the Cardiovascular System: A Scientific Statement From the American Heart Association. Circ. Res. 119, e39-e75.

Grimm, F.A., Iwata, Y., Sirenko, O., Bittner, M., and Rusyn, I. (2015). High-Content Assay Multiplexing for Toxicity Screening in Induced Pluripotent Stem Cell-Derived Cardiomyocytes and Hepatocytes. Assay Drug Dev. Technol. 13, 529-546.

Groarke, J.D., and Nohria, A. (2015). Anthracycline Cardiotoxicity. Circulation 131, 19461949.

Gu, J., Hu, W., and Zhang, D. (2015). Resveratrol, a polyphenol phytoalexin, protects against doxorubicin-induced cardiotoxicity. J. Cell. Mol. Med. 19, 2324-2328.

Guan, K., Nayernia, K., Maier, L.S., Wagner, S., Dressel, R., Lee, J.H., Nolte, J., Wolf, F., Li, M., Engel, W., et al. (2006). Pluripotency of spermatogonial stem cells from adult mouse testis. Nature 440, 1199-1203.

Guemez-Gamboa, A., Estrada-Sánchez, A.M., Montiel, T., Páramo, B., Massieu, L., and Morán, J. (2011). Activation of NOX2 by the stimulation of ionotropic and metabotropic glutamate receptors contributes to glutamate neurotoxicity in vivo through the production of reactive oxygen species and calpain activation. J. Neuropathol. Exp. Neurol. 70, 1020-1035.

Gutscher, M., Pauleau, A.-L., Marty, L., Brach, T., Wabnitz, G.H., Samstag, Y., Meyer, A.J., and Dick, T.P. (2008). Real-time imaging of the intracellular glutathione redox potential. Nat. Methods 5, 553-559.

Gutscher, M., Sobotta, M.C., Wabnitz, G.H., Ballikaya, S., Meyer, A.J., Samstag, Y., and Dick, T.P. (2009). Proximity-based Protein Thiol Oxidation by $\mathrm{H}_{2} \mathrm{O}_{2}$-scavenging Peroxidases. J. Biol. Chem. 284, 31532-31540.

Guzik, T.J., West, N.E.J., Black, E., McDonald, D., Ratnatunga, C., Pillai, R., and Channon, K.M. (2000). Functional Effect of the C242T Polymorphism in the NAD(P)H Oxidase p22phox Gene on Vascular Superoxide Production in Atherosclerosis. Circulation 102, 17441747.

Hanna, A.D., Janczura, M., Cho, E., Dulhunty, A.F., and Beard, N.A. (2011). Multiple actions of the anthracycline daunorubicin on cardiac ryanodine receptors. Mol. Pharmacol. 80, 538549.

Hanna, A.D., Lam, A., Tham, S., Dulhunty, A.F., and Beard, N.A. (2014). Adverse Effects of Doxorubicin and Its Metabolic Product on Cardiac RyR2 and SERCA2A. Mol. Pharmacol. 86, 438-449.

Hanna, J., Cheng, A.W., Saha, K., Kim, J., Lengner, C.J., Soldner, F., Cassady, J.P., Muffat, J., Carey, B.W., and Jaenisch, R. (2010a). Human embryonic stem cells with biological and epigenetic characteristics similar to those of mouse ESCs. Proc. Natl. Acad. Sci. U. S. A. 107, 9222-9227. 
Hanna, J.H., Saha, K., and Jaenisch, R. (2010b). Pluripotency and cellular reprogramming: facts, hypotheses, unresolved issues. Cell 143, 508-525.

Hasinoff, B.B., Hellmann, K., Herman, E.H., and Ferrans, V.J. (1998). Chemical, biological and clinical aspects of dexrazoxane and other bisdioxopiperazines. Curr. Med. Chem. 5, 1-28.

Hasinoff, B.B., Schroeder, P.E., and Patel, D. (2003). The Metabolites of the Cardioprotective Drug Dexrazoxane Do Not Protect Myocytes from Doxorubicin-Induced Cytotoxicity. Mol. Pharmacol. 64, 670-678.

Hattori, F., Chen, H., Yamashita, H., Tohyama, S., Satoh, Y.-S., Yuasa, S., Li, W., Yamakawa, H., Tanaka, T., Onitsuka, T., et al. (2010). Nongenetic method for purifying stem cell-derived cardiomyocytes. Nat. Methods 7, 61-66.

He, B.J., Joiner, M.-L.A., Singh, M.V., Luczak, E.D., Swaminathan, P.D., Koval, O.M., Kutschke, W., Allamargot, C., Yang, J., Guan, X., et al. (2011). Oxidation of CaMKII determines the cardiotoxic effects of aldosterone. Nat. Med. 17, 1610-1618.

He, J.-Q., Ma, Y., Lee, Y., Thomson, J.A., and Kamp, T.J. (2003). Human embryonic stem cells develop into multiple types of cardiac myocytes: action potential characterization. Circ. Res. 93, 32-39.

Heo, I., Joo, C., Kim, Y.-K., Ha, M., Yoon, M.-J., Cho, J., Yeom, K.-H., Han, J., and Kim, V.N. (2009). TUT4 in concert with Lin28 suppresses microRNA biogenesis through premicroRNA uridylation. Cell 138, 696-708.

Herman, E.H., Zhang, J., Chadwick, D.P., and Ferrans, V.J. (2000). Comparison of the protective effects of amifostine and dexrazoxane against the toxicity of doxorubicin in spontaneously hypertensive rats. Cancer Chemother. Pharmacol. 45, 329-334.

Heta, E. (2017). Development of a genetically encoded model for the sensing of glutathione redox potential in human embryonic stem cell-derived cardiomyocytes and fibroblasts.

Dissertation ediss.uni-goettingen.de.

Hochster, H., Liebes, L., Wadler, S., Oratz, R., Wernz, J.C., Meyers, M., Green, M., Blum, R.H., and Speyer, J.L. (1992). Pharmacokinetics of the cardioprotector ADR-529 (ICRF-187) in escalating doses combined with fixed-dose doxorubicin. J. Natl. Cancer Inst. 84, 17251730.

Holmgren, G., Synnergren, J., Bogestål, Y., Améen, C., Åkesson, K., Holmgren, S., Lindahl, A., and Sartipy, P. (2015). Identification of novel biomarkers for doxorubicin-induced toxicity in human cardiomyocytes derived from pluripotent stem cells. Toxicology 328, 102-111.

Hom, J.R., Quintanilla, R.A., Hoffman, D.L., de Mesy Bentley, K.L., Molkentin, J.D., Sheu, S.-S., and Porter, G.A. (2011). The permeability transition pore controls cardiac mitochondrial maturation and myocyte differentiation. Dev. Cell 21, 469-478.

Hong, J.H., Okada, K., Kusano, T., Komazawa, Y., Kobayashi, M., Mizutani, A., Kamada, N., and Kuramoto, A. (1990). Reduced DNA topoisomerase II in VP-16 resistant mouse breast cancer cell line. Biomed. Pharmacother. Biomedecine Pharmacother. 44, 41-45. 
Horie, T., Ono, K., Nishi, H., Nagao, K., Kinoshita, M., Watanabe, S., Kuwabara, Y., Nakashima, Y., Takanabe-Mori, R., Nishi, E., et al. (2010). Acute doxorubicin cardiotoxicity is associated with miR-146a-induced inhibition of the neuregulin-ErbB pathway. Cardiovasc. Res. 87, 656-664.

Hotta, A., and Ellis, J. (2008). Retroviral vector silencing during iPS cell induction: an epigenetic beacon that signals distinct pluripotent states. J. Cell. Biochem. 105, 940-948.

Hove-Madsen, L., and Bers, D.M. (1993). Sarcoplasmic reticulum Ca2+ uptake and thapsigargin sensitivity in permeabilized rabbit and rat ventricular myocytes. Circ. Res. 73, 820-828.

Hwang, H., Arcidi, J.M., Hale, S.L., Simkhovich, B.Z., Belardinelli, L., Dhalla, A.K., Shryock, J.C., and Kloner, R.A. (2009). Ranolazine as a cardioplegia additive improves recovery of diastolic function in isolated rat hearts. Circulation 120, S16-21.

Hwang, H.S., Kryshtal, D.O., Feaster, T.K., Sánchez-Freire, V., Zhang, J., Kamp, T.J., Hong, C.C., Wu, J.C., and Knollmann, B.C. (2015). Comparable Calcium Handling Of Human iPSC-derived Cardiomyocytes Generated By Multiple Laboratories. J. Mol. Cell. Cardiol. 85, 79-88.

Ichihara, S., Yamada, Y., Kawai, Y., Osawa, T., Furuhashi, K., Duan, Z., and Ichihara, G. (2007). Roles of oxidative stress and Akt signaling in doxorubicin cardiotoxicity. Biochem. Biophys. Res. Commun. 359, 27-33.

Ichikawa, Y., Ghanefar, M., Bayeva, M., Wu, R., Khechaduri, A., Prasad, S.V.N., Mutharasan, R.K., Naik, T.J., and Ardehali, H. (2014). Cardiotoxicity of doxorubicin is mediated through mitochondrial iron accumulation. J. Clin. Invest. 124, 617-630.

Ito, H., Miller, S.C., Billingham, M.E., Akimoto, H., Torti, S.V., Wade, R., Gahlmann, R., Lyons, G., Kedes, L., and Torti, F.M. (1990). Doxorubicin selectively inhibits muscle gene expression in cardiac muscle cells in vivo and in vitro. Proc. Natl. Acad. Sci. U. S. A. 87, 4275-4279.

Jaenisch, R., and Young, R. (2008). Stem cells, the molecular circuitry of pluripotency and nuclear reprogramming. Cell 132, 567-582.

Jang, Y.M., Kendaiah, S., Drew, B., Phillips, T., Selman, C., Julian, D., and Leeuwenburgh, C. (2004). Doxorubicin treatment in vivo activates caspase-12 mediated cardiac apoptosis in both male and female rats. FEBS Lett. 577, 483-490.

Jeyaseelan, R., Poizat, C., Baker, R.K., Abdishoo, S., Isterabadi, L.B., Lyons, G.E., and Kedes, L. (1997). A Novel Cardiac-Restricted Target for Doxorubicin CARP, A Nuclear Modulator Of Gene Expression In Cardiac Progenitor Cells And Cardiomyocytes. J. Biol. Chem. 272, 22800-22808.

Jung, C.B., Moretti, A., Mederos y Schnitzler, M., Iop, L., Storch, U., Bellin, M., Dorn, T., Ruppenthal, S., Pfeiffer, S., Goedel, A., et al. (2012). Dantrolene rescues arrhythmogenic 
RYR2 defect in a patient-specific stem cell model of catecholaminergic polymorphic ventricular tachycardia. EMBO Mol. Med. 4, 180-191.

Kaiserová, H., den Hartog, G.J.M., Šimůnek, T., Schröterová, L., Kvasničková, E., and Bast, A. (2006). Iron is not involved in oxidative stress-mediated cytotoxicity of doxorubicin and bleomycin. Br. J. Pharmacol. 149, 920-930.

Kalivendi, S.V., Konorev, E.A., Cunningham, S., Vanamala, S.K., Kaji, E.H., Joseph, J., and Kalyanaraman, B. (2005). Doxorubicin activates nuclear factor of activated T-lymphocytes and Fas ligand transcription: role of mitochondrial reactive oxygen species and calcium. Biochem. J. 389, 527-539.

Kanatsu-Shinohara, M., Inoue, K., Lee, J., Yoshimoto, M., Ogonuki, N., Miki, H., Baba, S., Kato, T., Kazuki, Y., Toyokuni, S., et al. (2004). Generation of pluripotent stem cells from neonatal mouse testis. Cell 119, 1001-1012.

Kelly, S.J. (1977). Studies of the developmental potential of 4- and 8-cell stage mouse blastomeres. J. Exp. Zool. 200, 365-376.

Khalil, P.N., Neuhof, C., Huss, R., Pollhammer, M., Khalil, M.N., Neuhof, H., Fritz, H., and Siebeck, M. (2005). Calpain inhibition reduces infarct size and improves global hemodynamics and left ventricular contractility in a porcine myocardial ischemia/reperfusion model. Eur. J. Pharmacol. 528, 124-131.

Kim, D.H., Landry, A.B., Lee, Y.S., and Katz, A.M. (1989). Doxorubicin-induced calcium release from cardiac sarcoplasmic reticulum vesicles. J. Mol. Cell. Cardiol. 21, 433-436.

Kim, D.-S., Woo, E.-R., Chae, S.-W., Ha, K.-C., Lee, G.-H., Hong, S.-T., Kwon, D.-Y., Kim, M.-S., Jung, Y.-K., Kim, H.-M., et al. (2007). Plantainoside D protects adriamycin-induced apoptosis in H9c2 cardiac muscle cells via the inhibition of ROS generation and NF-kappaB activation. Life Sci. 80, 314-323.

Kim, J., Chu, J., Shen, X., Wang, J., and Orkin, S.H. (2008). An extended transcriptional network for pluripotency of embryonic stem cells. Cell 132, 1049-1061.

Kim, S.-Y., Kim, S.-J., Kim, B.-J., Rah, S.-Y., Chung, S.M., Im, M.-J., and Kim, U.-H. (2006). Doxorubicin-induced reactive oxygen species generation and intracellular Ca2+ increase are reciprocally modulated in rat cardiomyocytes. Exp. Mol. Med. 38, 535-545.

Kimura, T., Fujita, I., Itoh, N., Muto, N., Nakanishi, T., Takahashi, K., Azuma, J., and Tanaka, K. (2000). Metallothionein acts as a cytoprotectant against doxorubicin toxicity. J. Pharmacol. Exp. Ther. 292, 299-302.

Kleinsmith, L.J., and Pierce, G.B. (1964). Multipotentiality of single embryonal carcinoma cells. Cancer Res. 24, 1544-1551.

Kolanowski, T.J., Antos, C.L., and Guan, K. (2017). Making human cardiomyocytes up to date: Derivation, maturation state and perspectives. Int. J. Cardiol. 
Koyanagi-Aoi, M., Ohnuki, M., Takahashi, K., Okita, K., Noma, H., Sawamura, Y., Teramoto, I., Narita, M., Sato, Y., Ichisaka, T., et al. (2013). Differentiation-defective phenotypes revealed by large-scale analyses of human pluripotent stem cells. Proc. Natl. Acad. Sci. U. S. A. 110, 20569-20574.

Kuroda, J., and Sadoshima, J. (2010). NADPH Oxidase and Cardiac Failure. J Cardiovasc. Transl. Res. 3, 314-320.

Kuroda, T., Yasuda, S., Kusakawa, S., Hirata, N., Kanda, Y., Suzuki, K., Takahashi, M., Nishikawa, S.-I., Kawamata, S., and Sato, Y. (2012). Highly sensitive in vitro methods for detection of residual undifferentiated cells in retinal pigment epithelial cells derived from human iPS cells. PloS One 7, e37342.

Kwon, S.H., Pimentel, D.R., Remondino, A., Sawyer, D.B., and Colucci, W.S. (2003). $\mathrm{H}(2) \mathrm{O}(2)$ regulates cardiac myocyte phenotype via concentration-dependent activation of distinct kinase pathways. J. Mol. Cell. Cardiol. 35, 615-621.

van Laake, L.W., Passier, R., Monshouwer-Kloots, J., Verkleij, A.J., Lips, D.J., Freund, C., den Ouden, K., Ward-van Oostwaard, D., Korving, J., Tertoolen, L.G., et al. (2007). Human embryonic stem cell-derived cardiomyocytes survive and mature in the mouse heart and transiently improve function after myocardial infarction. Stem Cell Res. 1, 9-24.

Laflamme, M.A., Chen, K.Y., Naumova, A.V., Muskheli, V., Fugate, J.A., Dupras, S.K., Reinecke, H., Xu, C., Hassanipour, M., Police, S., et al. (2007). Cardiomyocytes derived from human embryonic stem cells in pro-survival factors enhance function of infarcted rat hearts. Nat. Biotechnol. 25, 1015-1024.

Lassègue, B., San Martín, A., and Griendling, K.K. (2012). Biochemistry, physiology, and pathophysiology of NADPH oxidases in the cardiovascular system. Circ. Res. 110, 13641390.

Laurila, E., Ahola, A., Hyttinen, J., and Aalto-Setälä, K. (2016). Methods for in vitro functional analysis of iPSC derived cardiomyocytes - Special focus on analyzing the mechanical beating behavior. Biochim. Biophys. Acta BBA - Mol. Cell Res. 1863, 18641872.

Lefrak, E.A., Pitha, J., Rosenheim, S., and Gottlieb, J.A. (1973). A clinicopathologic analysis of adriamycin cardiotoxicity. Cancer 32, 302-314.

Legha, S.S., Wang, Y.M., Mackay, B., Ewer, M., Hortobagyi, G.N., Benjamin, R.S., and Ali, M.K. (1982). Clinical and pharmacologic investigation of the effects of alpha-tocopherol on adriamycin cardiotoxicity. Ann. N. Y. Acad. Sci. 393, 411-418.

Leong, S.L., Chaiyakunapruk, N., and Lee, S.W.H. (2017). Candidate Gene Association Studies of Anthracycline-induced Cardiotoxicity: A Systematic Review and Meta-analysis. Sci. Rep. 7, 39.

Li, T., Danelisen, I., and Singal, P.K. (2002). Early changes in myocardial antioxidant enzymes in rats treated with adriamycin. Mol. Cell. Biochem. 232, 19-26. 
Li, X., Li, Y., Shan, L., Shen, E., Chen, R., and Peng, T. (2009a). Over-expression of calpastatin inhibits calpain activation and attenuates myocardial dysfunction during endotoxaemia. Cardiovasc. Res. 83, 72-79.

Li, Y., Zhu, H., Kuppusamy, P., Roubaud, V., Zweier, J.L., and Trush, M.A. (1998). Validation of lucigenin (bis-N-methylacridinium) as a chemilumigenic probe for detecting superoxide anion radical production by enzymatic and cellular systems. J. Biol. Chem. 273, 2015-2023.

Li, Y., Arnold, J.M.O., Pampillo, M., Babwah, A.V., and Peng, T. (2009b). Taurine prevents cardiomyocyte death by inhibiting NADPH oxidase-mediated calpain activation. Free Radic. Biol. Med. 46, 51-61.

Li, Y., Li, Y., Feng, Q., Arnold, M., and Peng, T. (2009c). Calpain activation contributes to hyperglycaemia-induced apoptosis in cardiomyocytes. Cardiovasc. Res. 84, 100-110.

Li, Y., Ma, J., Zhu, H., Singh, M., Hill, D., Greer, P.A., Arnold, J.M., Abel, E.D., and Peng, T. (2011). Targeted inhibition of calpain reduces myocardial hypertrophy and fibrosis in mouse models of type 1 diabetes. Diabetes 60, 2985-2994.

Lian, X., Hsiao, C., Wilson, G., Zhu, K., Hazeltine, L.B., Azarin, S.M., Raval, K.K., Zhang, J., Kamp, T.J., and Palecek, S.P. (2012). Robust cardiomyocyte differentiation from human pluripotent stem cells via temporal modulation of canonical Wnt signaling. Proc. Natl. Acad. Sci. U. S. A. 109, E1848-1857.

Lian, X., Zhang, J., Azarin, S.M., Zhu, K., Hazeltine, L.B., Bao, X., Hsiao, C., Kamp, T.J., and Palecek, S.P. (2013). Directed cardiomyocyte differentiation from human pluripotent stem cells by modulating Wnt/ $\beta$-catenin signaling under fully defined conditions. Nat. Protoc. 8 , 162-175.

Lim, C.C., Zuppinger, C., Guo, X., Kuster, G.M., Helmes, M., Eppenberger, H.M., Suter, T.M., Liao, R., and Sawyer, D.B. (2004). Anthracyclines induce calpain-dependent titin proteolysis and necrosis in cardiomyocytes. J. Biol. Chem. 279, 8290-8299.

Lipshultz, S.E., Lipsitz, S.R., Mone, S.M., Goorin, A.M., Sallan, S.E., Sanders, S.P., Orav, E.J., Gelber, R.D., and Colan, S.D. (1995). Female Sex and Higher Drug Dose as Risk Factors for Late Cardiotoxic Effects of Doxorubicin Therapy for Childhood Cancer. N. Engl. J. Med. 332, 1738-1744.

Lipshultz, S.E., Rifai, N., Dalton, V.M., Levy, D.E., Silverman, L.B., Lipsitz, S.R., Colan, S.D., Asselin, B.L., Barr, R.D., Clavell, L.A., et al. (2004). The effect of dexrazoxane on myocardial injury in doxorubicin-treated children with acute lymphoblastic leukemia. N. Engl. J. Med. 351, 145-153.

Lipshultz, S.E., Scully, R.E., Lipsitz, S.R., Sallan, S.E., Silverman, L.B., Miller, T.L., Barry, E.V., Asselin, B.L., Athale, U., Clavell, L.A., et al. (2010). Assessment of dexrazoxane as a cardioprotectant in doxorubicin-treated children with high-risk acute lymphoblastic leukaemia: long-term follow-up of a prospective, randomised, multicentre trial. Lancet Oncol. 11, 950-961. 
Liu, F.-F., Stone, J.R., Schuldt, A.J.T., Okoshi, K., Okoshi, M.P., Nakayama, M., Ho, K.K.L., Manning, W.J., Marchionni, M.A., Lorell, B.H., et al. (2005). Heterozygous knockout of neuregulin-1 gene in mice exacerbates doxorubicin-induced heart failure. Am. J. Physiol. Heart Circ. Physiol. 289, H660-H666.

Liu, Y., Asnani, A., Zou, L., Bentley, V.L., Yu, M., Wang, Y., Dellaire, G., Sarkar, K.S., Dai, M., Chen, H.H., et al. (2014). Visnagin protects against doxorubicin-induced cardiomyopathy through modulation of mitochondrial malate dehydrogenase. Sci. Transl. Med. 6, 266ra170.

Looi, Y.H., Grieve, D.J., Siva, A., Walker, S.J., Anilkumar, N., Cave, A.C., Marber, M., Monaghan, M.J., and Shah, A.M. (2008). Involvement of Nox2 NADPH Oxidase in Adverse Cardiac Remodeling After Myocardial Infarction. Hypertension 51, 319-325.

Lopes, L.R., Dagher, M.-C., Gutierrez, A., Young, B., Bouin, A.-P., Fuchs, A., and Babior, B.M. (2004). Phosphorylated p40PHOX as a Negative Regulator of NADPH Oxidase. Biochemistry (Mosc.) 43, 3723-3730.

Lotrionte, M., Biondi-Zoccai, G., Abbate, A., Lanzetta, G., D’Ascenzo, F., Malavasi, V., Peruzzi, M., Frati, G., and Palazzoni, G. (2013). Review and meta-analysis of incidence and clinical predictors of anthracycline cardiotoxicity. Am. J. Cardiol. 112, 1980-1984.

Lundby, A., Andersen, M.N., Steffensen, A.B., Horn, H., Kelstrup, C.D., Francavilla, C., Jensen, L.J., Schmitt, N., Thomsen, M.B., and Olsen, J.V. (2013). In vivo phosphoproteomics analysis reveals the cardiac targets of $\beta$-adrenergic receptor signaling. Sci. Signal. 6, rs11.

Lundy, S.D., Zhu, W.-Z., Regnier, M., and Laflamme, M.A. (2013). Structural and functional maturation of cardiomyocytes derived from human pluripotent stem cells. Stem Cells Dev. 22, 1991-2002.

Ma, J., Guo, L., Fiene, S.J., Anson, B.D., Thomson, J.A., Kamp, T.J., Kolaja, K.L., Swanson, B.J., and January, C.T. (2011). High purity human-induced pluripotent stem cell-derived cardiomyocytes: electrophysiological properties of action potentials and ionic currents. Am. J. Physiol. Heart Circ. Physiol. 301, H2006-2017.

Ma, J., Wang, Y., Zheng, D., Wei, M., Xu, H., and Peng, T. (2013). Rac1 signalling mediates doxorubicin-induced cardiotoxicity through both reactive oxygen species-dependent and -independent pathways. Cardiovasc. Res. 97, 77-87.

Magdy, T., Burmeister, B.T., and Burridge, P.W. (2016). Validating the pharmacogenomics of chemotherapy-induced cardiotoxicity: What is missing? Pharmacol. Ther. 168, 113-125.

Maillet, A., Tan, K., Chai, X., Sadananda, S.N., Mehta, A., Ooi, J., Hayden, M.R., Pouladi, M.A., Ghosh, S., Shim, W., et al. (2016). Modeling Doxorubicin-Induced Cardiotoxicity in Human Pluripotent Stem Cell Derived-Cardiomyocytes. Sci. Rep. 6.

Martin, G.R. (1981). Isolation of a pluripotent cell line from early mouse embryos cultured in medium conditioned by teratocarcinoma stem cells. Proc. Natl. Acad. Sci. U. S. A. 78, 76347638. 
Martin, E., Thougaard, A.V., Grauslund, M., Jensen, P.B., Bjorkling, F., Hasinoff, B.B., Tjørnelund, J., Sehested, M., and Jensen, L.H. (2009). Evaluation of the topoisomerase IIinactive bisdioxopiperazine ICRF-161 as a protectant against doxorubicin-induced cardiomyopathy. Toxicology 255, 72-79.

Marx, S.O., Reiken, S., Hisamatsu, Y., Jayaraman, T., Burkhoff, D., Rosemblit, N., and Marks, A.R. (2000). PKA phosphorylation dissociates FKBP12.6 from the calcium release channel (ryanodine receptor): defective regulation in failing hearts. Cell 101, 365-376.

Mason, C., and Dunnill, P. (2008). A brief definition of regenerative medicine. Regen. Med. 3, $1-5$.

Masui, S., Nakatake, Y., Toyooka, Y., Shimosato, D., Yagi, R., Takahashi, K., Okochi, H., Okuda, A., Matoba, R., Sharov, A.A., et al. (2007). Pluripotency governed by Sox2 via regulation of Oct3/4 expression in mouse embryonic stem cells. Nat. Cell Biol. 9, 625-635.

Matsui, Y., Zsebo, K., and Hogan, B.L. (1992). Derivation of pluripotential embryonic stem cells from murine primordial germ cells in culture. Cell 70, 841-847.

Maurea, N., Coppola, C., Piscopo, G., Riccio, G., Maurea, C., Caputo, R., Barbieri, A., Arra, C., and De Laurentiis, M. (2016). Ranolazine after doxorubicin treatment: Cardioprotective effects in vitro and in vivo. J. Clin. Oncol. 34, e23148-e23148.

McLaughlin, D., Zhao, Y., O’Neill, K.M., Edgar, K.S., Dunne, P.D., Kearney, A.M., Grieve, D.J., and McDermott, B.J. (2017). Signalling mechanisms underlying doxorubicin and Nox2 NADPH oxidase-induced cardiomyopathy: involvement of mitofusin-2. Br. J. Pharmacol. n/an/a.

Melton, C., Judson, R.L., and Blelloch, R. (2010). Opposing microRNA families regulate selfrenewal in mouse embryonic stem cells. Nature 463, 621-626.

Menasché, P., Vanneaux, V., Hagège, A., Bel, A., Cholley, B., Cacciapuoti, I., Parouchev, A., Benhamouda, N., Tachdjian, G., Tosca, L., et al. (2015a). Human embryonic stem cell-derived cardiac progenitors for severe heart failure treatment: first clinical case report. Eur. Heart J. 36, 2011-2017.

Menasché, P., Vanneaux, V., Fabreguettes, J.-R., Bel, A., Tosca, L., Garcia, S., Bellamy, V., Farouz, Y., Pouly, J., Damour, O., et al. (2015b). Towards a clinical use of human embryonic stem cell-derived cardiac progenitors: a translational experience. Eur. Heart J. 36, 743-750.

Meyer, A.J., and Dick, T.P. (2010). Fluorescent protein-based redox probes. Antioxid. Redox Signal. 13, 621-650.

Meyer, A.J., Brach, T., Marty, L., Kreye, S., Rouhier, N., Jacquot, J.-P., and Hell, R. (2007). Redox-sensitive GFP in Arabidopsis thaliana is a quantitative biosensor for the redox potential of the cellular glutathione redox buffer. Plant J. Cell Mol. Biol. 52, 973-986.

Middleman, E., Luce, J., and Frei, E. (1971). Clinical trials with adriamycin. Cancer 28, 844850. 
Min, K., Kwon, O.-S., Smuder, A.J., Wiggs, M.P., Sollanek, K.J., Christou, D.D., Yoo, J.-K., Hwang, M.-H., Szeto, H.H., Kavazis, A.N., et al. (2015). Increased mitochondrial emission of reactive oxygen species and calpain activation are required for doxorubicin-induced cardiac and skeletal muscle myopathy. J. Physiol. 593, 2017-2036.

Minotti, G., Menna, P., Salvatorelli, E., Cairo, G., and Gianni, L. (2004). Anthracyclines: Molecular Advances and Pharmacologic Developments in Antitumor Activity and Cardiotoxicity. Pharmacol. Rev. 56, 185-229.

Mitsui, K., Tokuzawa, Y., Itoh, H., Segawa, K., Murakami, M., Takahashi, K., Maruyama, M., Maeda, M., and Yamanaka, S. (2003). The homeoprotein Nanog is required for maintenance of pluripotency in mouse epiblast and ES cells. Cell 113, 631-642.

Mohamed, T.M.A., Stone, N.R., Berry, E.C., Radzinsky, E., Huang, Y., Pratt, K., Ang, Y.-S., Yu, P., Wang, H., Tang, S., et al. (2016). Chemical Enhancement of In Vitro and In Vivo Direct Cardiac Reprogramming. Circulation.

Moldoveanu, T., Hosfield, C.M., Lim, D., Elce, J.S., Jia, Z., and Davies, P.L. (2002). A $\mathrm{Ca}(2+)$ switch aligns the active site of calpain. Cell 108, 649-660.

Mora, C., Serzanti, M., Consiglio, A., Memo, M., and Dell’Era, P. (2017). Clinical potentials of human pluripotent stem cells. Cell Biol. Toxicol. 33, 351-360.

Morgan, B., Sobotta, M.C., and Dick, T.P. (2011). Measuring EGSH and H2O2 with roGFP2based redox probes. Free Radic. Biol. Med. 51, 1943-1951.

Myers, C.E., McGuire, W.P., Liss, R.H., Ifrim, I., Grotzinger, K., and Young, R.C. (1977). Adriamycin: the role of lipid peroxidation in cardiac toxicity and tumor response. Science 197, 165-167.

Nabhan, C., Byrtek, M., Rai, A., Dawson, K., Zhou, X., Link, B.K., Friedberg, J.W., Zelenetz, A.D., Maurer, M.J., Cerhan, J.R., et al. (2015). Disease characteristics, treatment patterns, prognosis, outcomes and lymphoma-related mortality in elderly follicular lymphoma in the United States. Br. J. Haematol. 170, 85-95.

Nagy, A., Gócza, E., Diaz, E.M., Prideaux, V.R., Iványi, E., Markkula, M., and Rossant, J. (1990). Embryonic stem cells alone are able to support fetal development in the mouse. Dev. Camb. Engl. 110, 815-821.

National Institutes of Health (2016). NIH Stem Cell Information Home Page. In Stem Cell Information [World Wide Web site]. Bethesda, MD: National Institutes of Health, U.S. Department of Health and Human Services, 2016 [cited September 11, 2017] Available at $</ /$ stemcells.nih.gov/info/basics.htm>.

Nichols, J., and Smith, A. (2009). Naive and Primed Pluripotent States. Cell Stem Cell 4, 487-492.

Nichols, J., Zevnik, B., Anastassiadis, K., Niwa, H., Klewe-Nebenius, D., Chambers, I., Schöler, H., and Smith, A. (1998). Formation of pluripotent stem cells in the mammalian embryo depends on the POU transcription factor Oct4. Cell 95, 379-391. 
Nishimura, K., Sano, M., Ohtaka, M., Furuta, B., Umemura, Y., Nakajima, Y., Ikehara, Y., Kobayashi, T., Segawa, H., Takayasu, S., et al. (2011). Development of Defective and Persistent Sendai Virus Vector A Unique Gene Delivery/Expression System Ideal For Cell Reprogramming. J. Biol. Chem. 286, 4760-4771.

Nousiainen, T., Jantunen, E., Vanninen, E., and Hartikainen, J. (2002). Early decline in left ventricular ejection fraction predicts doxorubicin cardiotoxicity in lymphoma patients. Br. J. Cancer 86, 1697-1700.

O’Connor, M.D., Kardel, M.D., Iosfina, I., Youssef, D., Lu, M., Li, M.M., Vercauteren, S., Nagy, A., and Eaves, C.J. (2008). Alkaline phosphatase-positive colony formation is a sensitive, specific, and quantitative indicator of undifferentiated human embryonic stem cells. Stem Cells Dayt. Ohio 26, 1109-1116.

Octavia, Y., Tocchetti, C.G., Gabrielson, K.L., Janssens, S., Crijns, H.J., and Moens, A.L. (2012). Doxorubicin-induced cardiomyopathy: From molecular mechanisms to therapeutic strategies. J. Mol. Cell. Cardiol. 52, 1213-1225.

Ohkura, K., Lee, J.-D., Shimizu, H., Nakano, A., Uzui, H., Horikoshi, M., Fujibayashi, Y., Yonekura, Y., and Ueda, T. (2003). Mitochondrials complex I activity is reduced in latent adriamycin-induced cardiomyopathy of rat. Mol. Cell. Biochem. 248, 203-208.

Ojha, S., Al Taee, H., Goyal, S., Mahajan, U.B., Patil, C.R., Arya, D.S., and Rajesh, M. (2016). Cardioprotective Potentials of Plant-Derived Small Molecules against Doxorubicin Associated Cardiotoxicity.

Okita, K., Nakagawa, M., Hyenjong, H., Ichisaka, T., and Yamanaka, S. (2008). Generation of mouse induced pluripotent stem cells without viral vectors. Science 322, 949-953.

Oliveira, P.J., and Wallace, K.B. (2006). Depletion of adenine nucleotide translocator protein in heart mitochondria from doxorubicin-treated rats-Relevance for mitochondrial dysfunction. Toxicology 220, 160-168.

Olson, R.D., Li, X., Palade, P., Shadle, S.E., Mushlin, P.S., Gambliel, H.A., Fill, M., Boucek, R.J., and Cusack, B.J. (2000). Sarcoplasmic reticulum calcium release is stimulated and inhibited by daunorubicin and daunorubicinol. Toxicol. Appl. Pharmacol. 169, 168-176.

Ondrias, K., Borgatta, L., Kim, D.H., and Ehrlich, B.E. (1990). Biphasic effects of doxorubicin on the calcium release channel from sarcoplasmic reticulum of cardiac muscle. Circ. Res. 67, 1167-1174.

Østergaard, H., Tachibana, C., and Winther, J.R. (2004). Monitoring disulfide bond formation in the eukaryotic cytosol. J. Cell Biol. 166, 337-345.

Pal, R., Basu Thakur, P., Li, S., Minard, C., and Rodney, G.G. (2013). Real-time imaging of NADPH oxidase activity in living cells using a novel fluorescent protein reporter. PloS One 8 , e63989.

Panopoulos, A.D., Yanes, O., Ruiz, S., Kida, Y.S., Diep, D., Tautenhahn, R., Herrerías, A., Batchelder, E.M., Plongthongkum, N., Lutz, M., et al. (2012). The metabolome of induced 
pluripotent stem cells reveals metabolic changes occurring in somatic cell reprogramming. Cell Res. 22, 168-177.

Perrin, C., Ecarnot-Laubriet, A., Vergely, C., and Rochette, L. (2003). Calpain and caspase-3 inhibitors reduce infarct size and post-ischemic apoptosis in rat heart without modifying contractile recovery. Cell. Mol. Biol. Noisy--Gd. Fr. 49 Online Pub, OL497-505.

Pessah, I.N., Durie, E.L., Schiedt, M.J., and Zimanyi, I. (1990). Anthraquinone-sensitized $\mathrm{Ca} 2+$ release channel from rat cardiac sarcoplasmic reticulum: possible receptor-mediated mechanism of doxorubicin cardiomyopathy. Mol. Pharmacol. 37, 503-514.

Peters, M.F., Lamore, S.D., Guo, L., Scott, C.W., and Kolaja, K.L. (2015). Human stem cellderived cardiomyocytes in cellular impedance assays: bringing cardiotoxicity screening to the front line. Cardiovasc. Toxicol. 15, 127-139.

Pfreundschuh, M., Schubert, J., Ziepert, M., Schmits, R., Mohren, M., Lengfelder, E., Reiser, M., Nickenig, C., Clemens, M., Peter, N., et al. (2008). Six versus eight cycles of bi-weekly CHOP-14 with or without rituximab in elderly patients with aggressive CD20+ B-cell lymphomas: a randomised controlled trial (RICOVER-60). Lancet Oncol. 9, 105-116.

Piacentino, V., Weber, C.R., Chen, X., Weisser-Thomas, J., Margulies, K.B., Bers, D.M., and Houser, S.R. (2003). Cellular Basis of Abnormal Calcium Transients of Failing Human Ventricular Myocytes. Circ. Res. 92, 651-658.

Protze, S.I., Liu, J., Nussinovitch, U., Ohana, L., Backx, P.H., Gepstein, L., and Keller, G.M. (2017). Sinoatrial node cardiomyocytes derived from human pluripotent cells function as a biological pacemaker. Nat. Biotechnol. 35, 56-68.

Reichwagen, A., Ziepert, M., Kreuz, M., Gödtel-Armbrust, U., Rixecker, T., Poeschel, V., Reza Toliat, M., Nürnberg, P., Tzvetkov, M., Deng, S., et al. (2015). Association of NADPH oxidase polymorphisms with anthracycline-induced cardiotoxicity in the RICOVER-60 trial of patients with aggressive CD20(+) B-cell lymphoma. Pharmacogenomics 16, 361-372.

Ren, Y., Lee, M.Y., Schliffke, S., Paavola, J., Amos, P.J., Ge, X., Ye, M., Zhu, S., Senyei, G., Lum, L., et al. (2011). Small molecule Wnt inhibitors enhance the efficiency of BMP-4directed cardiac differentiation of human pluripotent stem cells. J. Mol. Cell. Cardiol. 51, 280-287.

Rezende, F., Löwe, O., Helfinger, V., Prior, K.-K., Walter, M., Zukunft, S., Fleming, I., Weissmann, N., Brandes, R.P., and Schröder, K. (2016). Unchanged NADPH Oxidase Activity in Nox1-Nox2-Nox4 Triple Knockout Mice: What Do NADPH-Stimulated Chemiluminescence Assays Really Detect? Antioxid. Redox Signal. 24, 392-399.

Robert, J., and Gianni, L. (1993). Pharmacokinetics and metabolism of anthracyclines. Cancer Surv. 17, 219-252.

Røe, Å.T., Frisk, M., and Louch, W.E. (2015). Targeting Cardiomyocyte Ca2+ Homeostasis in Heart Failure. Curr. Pharm. Des. 21, 431-448. 
Rota, C., Chignell, C.F., and Mason, R.P. (1999a). Evidence for free radical formation during the oxidation of 2'-7'-dichlorofluorescin to the fluorescent dye 2'-7'-dichlorofluorescein by horseradish peroxidase:: Possible implications for oxidative stress measurements. Free Radic. Biol. Med. 27, 873-881.

Rota, C., Fann, Y.C., and Mason, R.P. (1999b). Phenoxyl Free Radical Formation during the Oxidation of the Fluorescent Dye 2',7'-Dichlorofluorescein by Horseradish Peroxidase Possible Concequences For Oxidative Stress Measurements. J. Biol. Chem. 274, 2816128168.

Rudzinski, T., Ciesielczyk, M., Religa, W., Bednarkiewicz, Z., and Krzeminska-Pakula, M. (2007). Doxorubicin-induced ventricular arrhythmia treated by implantation of an automatic cardioverter-defibrillator. Eur. Eur. Pacing Arrhythm. Card. Electrophysiol. J. Work. Groups Card. Pacing Arrhythm. Card. Cell. Electrophysiol. Eur. Soc. Cardiol. 9, 278-280.

Sabri, A., Hughie, H.H., and Lucchesi, P.A. (2003). Regulation of hypertrophic and apoptotic signaling pathways by reactive oxygen species in cardiac myocytes. Antioxid. Redox Signal. 5, 731-740.

Sag, C.M., Köhler, A.C., Anderson, M.E., Backs, J., and Maier, L.S. (2011). CaMKIIdependent SR Ca leak contributes to doxorubicin-induced impaired Ca handling in isolated cardiac myocytes. J. Mol. Cell. Cardiol. 51, 749-759.

Samavarchi-Tehrani, P., Golipour, A., David, L., Sung, H.-K., Beyer, T.A., Datti, A., Woltjen, K., Nagy, A., and Wrana, J.L. (2010). Functional genomics reveals a BMP-driven mesenchymal-to-epithelial transition in the initiation of somatic cell reprogramming. Cell Stem Cell 7, 64-77.

Santos, C.X.C., Raza, S., and Shah, A.M. (2016). Redox signaling in the cardiomyocyte: From physiology to failure. Int. J. Biochem. Cell Biol. 74, 145-151.

Sasaki, K., Makiyama, T., Yoshida, Y., Wuriyanghai, Y., Kamakura, T., Nishiuchi, S., Hayano, M., Harita, T., Yamamoto, Y., Kohjitani, H., et al. (2016). Patient-Specific Human Induced Pluripotent Stem Cell Model Assessed with Electrical Pacing Validates S107 as a Potential Therapeutic Agent for Catecholaminergic Polymorphic Ventricular Tachycardia. PloS One 11, e0164795.

Sathyamoorthy, M., de Mendez, I., Adams, A.G., and Leto, T.L. (1997). p40(phox) downregulates NADPH oxidase activity through interactions with its SH3 domain. J. Biol. Chem. 272, 9141-9146.

Schafer, F.Q., and Buettner, G.R. (2001). Redox environment of the cell as viewed through the redox state of the glutathione disulfide/glutathione couple. Free Radic. Biol. Med. 30, 1191-1212.

Schindelin, J., Arganda-Carreras, I., Frise, E., Kaynig, V., Longair, M., Pietzsch, T., Preibisch, S., Rueden, C., Saalfeld, S., Schmid, B., et al. (2012). Fiji: an open-source platform for biological-image analysis. Nat. Methods 9, 676-682. 
Schindelin, J., Rueden, C.T., Hiner, M.C., and Eliceiri, K.W. (2015). The ImageJ ecosystem: An open platform for biomedical image analysis. Mol. Reprod. Dev. 82, 518-529.

Schirmer, M., Hoffmann, M., Kaya, E., Tzvetkov, M., and Brockmöller, J. (2007). Genetic polymorphisms of NAD(P)H oxidase: variation in subunit expression and enzyme activity. Pharmacogenomics J. 8, 297-304.

Schneider, C.A., Rasband, W.S., and Eliceiri, K.W. (2012). NIH Image to ImageJ: 25 years of image analysis. Nat. Methods 9, 671-675.

Schopperle, W.M., and DeWolf, W.C. (2007). The TRA-1-60 and TRA-1-81 human pluripotent stem cell markers are expressed on podocalyxin in embryonal carcinoma. Stem Cells Dayt. Ohio 25, 723-730.

Serrano, J., Palmeira, C.M., Kuehl, D.W., and Wallace, K.B. (1999). Cardioselective and cumulative oxidation of mitochondrial DNA following subchronic doxorubicin administration. Biochim. Biophys. Acta BBA - Bioenerg. 1411, 201-205.

Shan, L., Li, J., Wei, M., Ma, J., Wan, L., Zhu, W., Li, Y., Zhu, H., Arnold, J.M.O., and Peng, T. (2010). Disruption of Rac1 signaling reduces ischemia-reperfusion injury in the diabetic heart by inhibiting calpain. Free Radic. Biol. Med. 49, 1804-1814.

Sheu, M.-L., Shen, C.-C., Chen, Y.-S., and Chiang, C.-K. (2017). Ochratoxin A induces ER stress and apoptosis in mesangial cells via a NADPH oxidase-derived reactive oxygen species-mediated calpain activation pathway. Oncotarget 8, 19376-19388.

Shiels, H.A., and Galli, G.L.J. (2014). The Sarcoplasmic Reticulum and the Evolution of the Vertebrate Heart. Physiology 29, 456-469.

Shimo-Nakanishi, Y., Hasebe, T., Suzuki, A., Mochizuki, H., Nomiyama, T., Tanaka, Y., Nagaoka, I., Mizuno, Y., and Urabe, T. (2004). Functional effects of NAD(P)H oxidase p22phox C242T mutation in human leukocytes and association with thrombotic cerebral infarction. Atherosclerosis 175, 109-115.

Siveski-Iliskovic, N., Hill, M., Chow, D.A., and Singal, P.K. (1995). Probucol protects against adriamycin cardiomyopathy without interfering with its antitumor effect. Circulation 91, 1015.

Siwik, D.A., Tzortzis, J.D., Pimental, D.R., Chang, D.L., Pagano, P.J., Singh, K., Sawyer, D.B., and Colucci, W.S. (1999). Inhibition of copper-zinc superoxide dismutase induces cell growth, hypertrophic phenotype, and apoptosis in neonatal rat cardiac myocytes in vitro. Circ. Res. 85, 147-153.

Skatchkov, M.P., Sperling, D., Hink, U., Mülsch, A., Harrison, D.G., Sindermann, I., Meinertz, T., and Münzel, T. (1999). Validation of lucigenin as a chemiluminescent probe to monitor vascular superoxide as well as basal vascular nitric oxide production. Biochem. Biophys. Res. Commun. 254, 319-324. 
Smith, L.A., Cornelius, V.R., Plummer, C.J., Levitt, G., Verrill, M., Canney, P., and Jones, A. (2010). Cardiotoxicity of anthracycline agents for the treatment of cancer: Systematic review and meta-analysis of randomised controlled trials. BMC Cancer 10, 337.

Someya, A., Nunoi, H., Hasebe, T., and Nagaoka, I. (1999). Phosphorylation of p40-phox during activation of neutrophil NADPH oxidase. J. Leukoc. Biol. 66, 851-857.

Soufi, A., Donahue, G., and Zaret, K.S. (2012). Facilitators and impediments of the pluripotency reprogramming factors’ initial engagement with the genome. Cell 151, 9941004.

Sparano, J.A., Speyer, J., Gradishar, W.J., Liebes, L., Sridhara, R., Mendoza, S., Fry, D., and Egorin, M.J. (1999). Phase I trial of escalating doses of paclitaxel plus doxorubicin and dexrazoxane in patients with advanced breast cancer. J. Clin. Oncol. Off. J. Am. Soc. Clin. Oncol. 17, 880-886.

Stadtfeld, M., Nagaya, M., Utikal, J., Weir, G., and Hochedlinger, K. (2008). Induced pluripotent stem cells generated without viral integration. Science 322, 945-949.

Steinberg, J.S., Cohen, A.J., Wasserman, A.G., Cohen, P., and Ross, A.M. (1987). Acute arrhythmogenicity of doxorubicin administration. Cancer 60, 1213-1218.

Steinherz, L.J., Steinherz, P.G., Tan, C.T., Heller, G., and Murphy, M.L. (1991). Cardiac toxicity 4 to 20 years after completing anthracycline therapy. JAMA 266, 1672-1677.

Štěrba, M., Popelová, O., Lenčo, J., Fučíková, A., Brčáková, E., Mazurová, Y., Jirkovský, E., Šimůnek, T., Adamcová, M., Mičuda, S., et al. (2011). Proteomic insights into chronic anthracycline cardiotoxicity. J. Mol. Cell. Cardiol. 50, 849-862.

Štěrba, M., Popelová, O., Vávrová, A., Jirkovský, E., Kovaříková, P., Geršl, V., and Šimůnek, T. (2013). Oxidative Stress, Redox Signaling, and Metal Chelation in Anthracycline Cardiotoxicity and Pharmacological Cardioprotection. Antioxid. Redox Signal. 18, 899-929.

Stevens, L.C., and Little, C.C. (1954). Spontaneous Testicular Teratomas in an Inbred Strain of Mice. Proc. Natl. Acad. Sci. U. S. A. 40, 1080-1087.

Streckfuss-Bömeke, K., Wolf, F., Azizian, A., Stauske, M., Tiburcy, M., Wagner, S., Hübscher, D., Dressel, R., Chen, S., Jende, J., et al. (2013). Comparative study of human-induced pluripotent stem cells derived from bone marrow cells, hair keratinocytes, and skin fibroblasts. Eur. Heart J. 34, 2618-2629.

Streckfuss-Bömeke, K., Tiburcy, M., Fomin, A., Luo, X., Li, W., Fischer, C., Özcelik, C., Perrot, A., Sossalla, S., Haas, J., et al. (2017). Severe DCM phenotype of patient harboring RBM20 mutation S635A can be modeled by patient-specific induced pluripotent stem cellderived cardiomyocytes. J. Mol. Cell. Cardiol. 113, 9-21.

Swain, L., Kesemeyer, A., Meyer-Roxlau, S., Vettel, C., Zieseniss, A., Güntsch, A., Jatho, A., Becker, A., Nanadikar, M.S., Morgan, B., et al. (2016). Redox Imaging Using Cardiac Myocyte-Specific Transgenic Biosensor Mice. Circ. Res. 119, 1004-1016. 
Swain, S.M., Whaley, F.S., Gerber, M.C., Weisberg, S., York, M., Spicer, D., Jones, S.E., Wadler, S., Desai, A., Vogel, C., et al. (1997). Cardioprotection with dexrazoxane for doxorubicin-containing therapy in advanced breast cancer. J. Clin. Oncol. 15, 1318-1332.

Swain, S.M., Whaley, F.S., and Ewer, M.S. (2003). Congestive heart failure in patients treated with doxorubicin. Cancer 97, 2869-2879.

Swaminathan, P.D., Purohit, A., Soni, S., Voigt, N., Singh, M.V., Glukhov, A.V., Gao, Z., He, B.J., Luczak, E.D., Joiner, M.A., et al. (2011). Oxidized CaMKII causes cardiac sinus node dysfunction in mice. J. Clin. Invest. 121, 3277-3288.

Tada, M., Takahama, Y., Abe, K., Nakatsuji, N., and Tada, T. (2001). Nuclear reprogramming of somatic cells by in vitro hybridization with ES cells. Curr. Biol. CB 11, 1553-1558.

Takahashi, K., and Yamanaka, S. (2006). Induction of pluripotent stem cells from mouse embryonic and adult fibroblast cultures by defined factors. Cell 126, 663-676.

Takahashi, K., and Yamanaka, S. (2016). A decade of transcription factor-mediated reprogramming to pluripotency. Nat. Rev. Mol. Cell Biol. 17, 183-193.

Takahashi, K., Tanabe, K., Ohnuki, M., Narita, M., Ichisaka, T., Tomoda, K., and Yamanaka, S. (2007). Induction of pluripotent stem cells from adult human fibroblasts by defined factors. Cell 131, 861-872.

Takashima, Y., Guo, G., Loos, R., Nichols, J., Ficz, G., Krueger, F., Oxley, D., Santos, F., Clarke, J., Mansfield, W., et al. (2014). Resetting transcription factor control circuitry toward ground-state pluripotency in human. Cell 158, 1254-1269.

Tamura, M., Shiozaki, I., Ono, S., Miyano, K., Kunihiro, S., and Sasaki, T. (2007). p40phox as an alternative organizer to p47phox in Nox2 activation: A new mechanism involving an interaction with p22phox. FEBS Lett. 581, 4533-4538.

Tester, D.J., and Ackerman, M.J. (2014). Genetics of long QT syndrome. Methodist DeBakey Cardiovasc. J. 10, 29-33.

Tewey, K.M., Rowe, T.C., Yang, L., Halligan, B.D., and Liu, L.F. (1984). Adriamycin-induced DNA damage mediated by mammalian DNA topoisomerase II. Science 226, 466-468.

Theunissen, T.W., Powell, B.E., Wang, H., Mitalipova, M., Faddah, D.A., Reddy, J., Fan, Z.P., Maetzel, D., Ganz, K., Shi, L., et al. (2014). Systematic Identification of Culture Conditions for Induction and Maintenance of Naive Human Pluripotency. Cell Stem Cell 15, 471-487.

Theunissen, T.W., Friedli, M., He, Y., Planet, E., O’Neil, R.C., Markoulaki, S., Pontis, J., Wang, H., Iouranova, A., Imbeault, M., et al. (2016). Molecular Criteria for Defining the Naive Human Pluripotent State. Cell Stem Cell 19, 502-515.

Thomson, J.A., Itskovitz-Eldor, J., Shapiro, S.S., Waknitz, M.A., Swiergiel, J.J., Marshall, V.S., and Jones, J.M. (1998). Embryonic stem cell lines derived from human blastocysts. Science 282, 1145-1147. 
Tiburcy, M., Hudson, J.E., Balfanz, P., Schlick, S., Meyer, T., Chang Liao, M.-L., Levent, E., Raad, F., Zeidler, S., Wingender, E., et al. (2017). Defined Engineered Human Myocardium With Advanced Maturation for Applications in Heart Failure Modeling and Repair. Circulation 135, 1832-1847.

Tocchetti, C.G., Carpi, A., Coppola, C., Quintavalle, C., Rea, D., Campesan, M., Arcari, A., Piscopo, G., Cipresso, C., Monti, M.G., et al. (2014). Ranolazine protects from doxorubicininduced oxidative stress and cardiac dysfunction. Eur. J. Heart Fail. 16, 358-366.

Tohyama, S., Hattori, F., Sano, M., Hishiki, T., Nagahata, Y., Matsuura, T., Hashimoto, H., Suzuki, T., Yamashita, H., Satoh, Y., et al. (2013). Distinct metabolic flow enables large-scale purification of mouse and human pluripotent stem cell-derived cardiomyocytes. Cell Stem Cell 12, 127-137.

Tohyama, S., Fujita, J., Hishiki, T., Matsuura, T., Hattori, F., Ohno, R., Kanazawa, H., Seki, T., Nakajima, K., Kishino, Y., et al. (2016). Glutamine Oxidation Is Indispensable for Survival of Human Pluripotent Stem Cells. Cell Metab. 23, 663-674.

Tracy, R.E., and Sander, G.E. (2011). Histologically Measured Cardiomyocyte Hypertrophy Correlates with Body Height as Strongly as with Body Mass Index. Cardiol. Res. Pract. 2011.

Uosaki, H., Fukushima, H., Takeuchi, A., Matsuoka, S., Nakatsuji, N., Yamanaka, S., and Yamashita, J.K. (2011). Efficient and Scalable Purification of Cardiomyocytes from Human Embryonic and Induced Pluripotent Stem Cells by VCAM1 Surface Expression. PLOS ONE 6, e23657.

Vásquez-Vivar, J., Hogg, N., Pritchard, K.A., Martasek, P., and Kalyanaraman, B. (1997). Superoxide anion formation from lucigenin: an electron spin resonance spin-trapping study. FEBS Lett. 403, 127-130.

Volkova, M., and Russell, R. (2011). Anthracycline Cardiotoxicity: Prevalence, Pathogenesis and Treatment. Curr. Cardiol. Rev. 7, 214-220.

Von Hoff, D.D., Layard, M.W., Basa, P., Davis, H.L., Von Hoff, A.L., Rozencweig, M., and Muggia, F.M. (1979). Risk factors for doxorubicin-induced congestive heart failure. Ann. Intern. Med. 91, 710-717.

Wagner, S., Dantz, C., Flebbe, H., Azizian, A., Sag, C.M., Engels, S., Möllencamp, J., Dybkova, N., Islam, T., Shah, A.M., et al. (2014). NADPH oxidase 2 mediates angiotensin IIdependent cellular arrhythmias via PKA and CaMKII. J. Mol. Cell. Cardiol. 75, 206-215.

Wallace, K.B. (2003). Doxorubicin-Induced Cardiac Mitochondrionopathy. Pharmacol. Toxicol. 93, 105-115.

Wang, J.H.-C., and Lin, J.-S. (2007). Cell traction force and measurement methods. Biomech. Model. Mechanobiol. 6, 361-371.

Wang, J., Gao, E., Rabinowitz, J., Song, J., Zhang, X.-Q., Koch, W.J., Tucker, A.L., Chan, T.O., Feldman, A.M., and Cheung, J.Y. (2011). Regulation of in vivo cardiac contractility by 
phospholemman: role of Na+/Ca2+ exchange. Am. J. Physiol. Heart Circ. Physiol. 300, H859-868.

Wang, S., Kotamraju, S., Konorev, E., Kalivendi, S., Joseph, J., and Kalyanaraman, B. (2002). Activation of nuclear factor-kappaB during doxorubicin-induced apoptosis in endothelial cells and myocytes is pro-apoptotic: the role of hydrogen peroxide. Biochem. J. 367, 729-740.

Wang, Y., Zheng, D., Wei, M., Ma, J., Yu, Y., Chen, R., Lacefield, J.C., Xu, H., and Peng, T. (2013). Over-expression of calpastatin aggravates cardiotoxicity induced by doxorubicin. Cardiovasc. Res. 98, 381-390.

Ware, C.B., Nelson, A.M., Mecham, B., Hesson, J., Zhou, W., Jonlin, E.C., Jimenez-Caliani, A.J., Deng, X., Cavanaugh, C., Cook, S., et al. (2014). Derivation of naïve human embryonic stem cells. Proc. Natl. Acad. Sci. U. S. A. 111, 4484-4489.

Weng, Z., Kong, C.-W., Ren, L., Karakikes, I., Geng, L., He, J., Chow, M.Z.Y., Mok, C.F., Keung, W., Chow, H., et al. (2014). A simple, cost-effective but highly efficient system for deriving ventricular cardiomyocytes from human pluripotent stem cells. Stem Cells Dev. 23, 1704-1716.

Wobus, A.M., Guan, K., Yang, H.T., and Boheler, K.R. (2002). Embryonic stem cells as a model to study cardiac, skeletal muscle, and vascular smooth muscle cell differentiation. Methods Mol. Biol. Clifton NJ 185, 127-156.

Wojnowski, L., Kulle, B., Schirmer, M., Schlüter, G., Schmidt, A., Rosenberger, A., Vonhof, S., Bickeböller, H., Toliat, M.R., Suk, E.-K., et al. (2005). NAD(P)H Oxidase and Multidrug Resistance Protein Genetic Polymorphisms Are Associated With Doxorubicin-Induced Cardiotoxicity. Circulation 112, 3754-3762.

Xie, H., Ye, M., Feng, R., and Graf, T. (2004). Stepwise reprogramming of B cells into macrophages. Cell 117, 663-676.

Xu, X., Persson, H.L., and Richardson, D.R. (2005). Molecular Pharmacology of the Interaction of Anthracyclines with Iron. Mol. Pharmacol. 68, 261-271.

Yamaoka, M., Yamaguchi, S., Suzuki, T., Okuyama, M., Nitobe, J., Nakamura, N., Mitsui, Y., and Tomoike, H. (2000). Apoptosis in rat cardiac myocytes induced by Fas ligand: priming for Fas-mediated apoptosis with doxorubicin. J. Mol. Cell. Cardiol. 32, 881-889.

Yancy, C.W., Jessup, M., Bozkurt, B., Butler, J., Casey, D.E., Drazner, M.H., Fonarow, G.C., Geraci, S.A., Horwich, T., Januzzi, J.L., et al. (2013). 2013 ACCF/AHA Guideline for the Management of Heart Failure: A Report of the American College of Cardiology Foundation/American Heart Association Task Force on Practice Guidelines. Circulation 128, e240-e327.

Yang, L., Soonpaa, M.H., Adler, E.D., Roepke, T.K., Kattman, S.J., Kennedy, M., Henckaerts, E., Bonham, K., Abbott, G.W., Linden, R.M., et al. (2008). Human cardiovascular progenitor cells develop from a KDR+ embryonic-stem-cell-derived population. Nature 453, 524-528. 
Yang, X., Pabon, L., and Murry, C.E. (2014a). Engineering Adolescence: Maturation of Human Pluripotent Stem Cell-Derived Cardiomyocytes. Circ. Res. 114, 511-523.

Yang, X., Pabon, L., and Murry, C.E. (2014b). Engineering Adolescence: Maturation of Human Pluripotent Stem Cell-Derived Cardiomyocytes. Circ. Res. 114, 511-523.

Yen, H.C., Oberley, T.D., Gairola, C.G., Szweda, L.I., and St Clair, D.K. (1999). Manganese superoxide dismutase protects mitochondrial complex I against adriamycin-induced cardiomyopathy in transgenic mice. Arch. Biochem. Biophys. 362, 59-66.

Yoshida, Y., and Yamanaka, S. (2017). Induced Pluripotent Stem Cells 10 Years Later: For Cardiac Applications. Circ. Res. 120, 1958-1968.

Yoshizawa, T., Takizawa, S., Shimada, S., Tokudome, T., Shindo, T., and Matsumoto, K. (2016). Effects of Adrenomedullin on Doxorubicin-Induced Cardiac Damage in Mice. Biol. Pharm. Bull. 39, 737-746.

Yu, J., Vodyanik, M.A., Smuga-Otto, K., Antosiewicz-Bourget, J., Frane, J.L., Tian, S., Nie, J., Jonsdottir, G.A., Ruotti, V., Stewart, R., et al. (2007). Induced pluripotent stem cell lines derived from human somatic cells. Science 318, 1917-1920.

Zalk, R., Lehnart, S.E., and Marks, A.R. (2007). Modulation of the ryanodine receptor and intracellular calcium. Annu. Rev. Biochem. 76, 367-385.

Zamorano, J.L., Lancellotti, P., Rodriguez Muñoz, D., Aboyans, V., Asteggiano, R., Galderisi, M., Habib, G., Lenihan, D.J., Lip, G.Y.H., Lyon, A.R., et al. (2016). 2016 ESC Position Paper on cancer treatments and cardiovascular toxicity developed under the auspices of the ESC Committee for Practice Guidelines: The Task Force for cancer treatments and cardiovascular toxicity of the European Society of Cardiology (ESC). Eur. Heart J. 37, 2768-2801.

Zhang, M., Perino, A., Ghigo, A., Hirsch, E., and Shah, A.M. (2013). NADPH Oxidases in Heart Failure: Poachers or Gamekeepers? Antioxid. Redox Signal. 18, 1024-1041.

Zhang, P., Andrianakos, R., Yang, Y., Liu, C., and Lu, W. (2010). Kruppel-like factor 4 (Klf4) prevents embryonic stem (ES) cell differentiation by regulating Nanog gene expression. J. Biol. Chem. 285, 9180-9189.

Zhang, S., Liu, X., Bawa-Khalfe, T., Lu, L.-S., Lyu, Y.L., Liu, L.F., and Yeh, E.T.H. (2012). Identification of the molecular basis of doxorubicin-induced cardiotoxicity. Nat. Med. 18, 1639-1642.

Zhang, W.Y., de Almeida, P.E., and Wu, J.C. (2008). Teratoma formation: A tool for monitoring pluripotency in stem cell research. In StemBook, (Cambridge (MA): Harvard Stem Cell Institute), p.

Zhang, Y., Chen, Y., Zhang, M., Tang, Y., Xie, Y., Huang, X., and Li, Y. (2014). Doxorubicin induces sarcoplasmic reticulum calcium regulation dysfunction via the decrease of SERCA2 and phospholamban expressions in rats. Cell Biochem. Biophys. 70, 1791-1798. 
Zhang, Y.-W., Shi, J., Li, Y.-J., and Wei, L. (2009). Cardiomyocyte death in doxorubicininduced cardiotoxicity. Arch. Immunol. Ther. Exp. (Warsz.) 57, 435-445.

Zhao, L., and Zhang, B. (2017). Doxorubicin induces cardiotoxicity through upregulation of death receptors mediated apoptosis in cardiomyocytes. Sci. Rep. 7.

Zhao, Y., McLaughlin, D., Robinson, E., Harvey, A.P., Hookham, M.B., Shah, A.M., McDermott, B.J., and Grieve, D.J. (2010). Nox2 NADPH Oxidase Promotes Pathologic Cardiac Remodeling Associated with Doxorubicin Chemotherapy. Cancer Res. 70, 92879297.

Zhao, Z., Fefelova, N., Shanmugam, M., Bishara, P., Babu, G.J., and Xie, L.-H. (2011). Angiotensin II induces afterdepolarizations via reactive oxygen species and calmodulin kinase II signaling. J. Mol. Cell. Cardiol. 50, 128-136.

Zhou, H., Wu, S., Joo, J.Y., Zhu, S., Han, D.W., Lin, T., Trauger, S., Bien, G., Yao, S., Zhu, Y., et al. (2009). Generation of induced pluripotent stem cells using recombinant proteins. Cell Stem Cell 4, 381-384.

Zielonka, J., and Kalyanaraman, B. (2010). Hydroethidine- and Mito-SOX-derived red fluorescence is not a reliable indicator of intracellular superoxide formation: Another inconvenient truth. Free Radic. Biol. Med. 48, 983-1001.

Zorzato, F., Margreth, A., and Volpe, P. (1986). Direct photoaffinity labeling of junctional sarcoplasmic reticulum with [14C]doxorubicin. J. Biol. Chem. 261, 13252-13257. 


\section{$7 \quad$ Appendix}

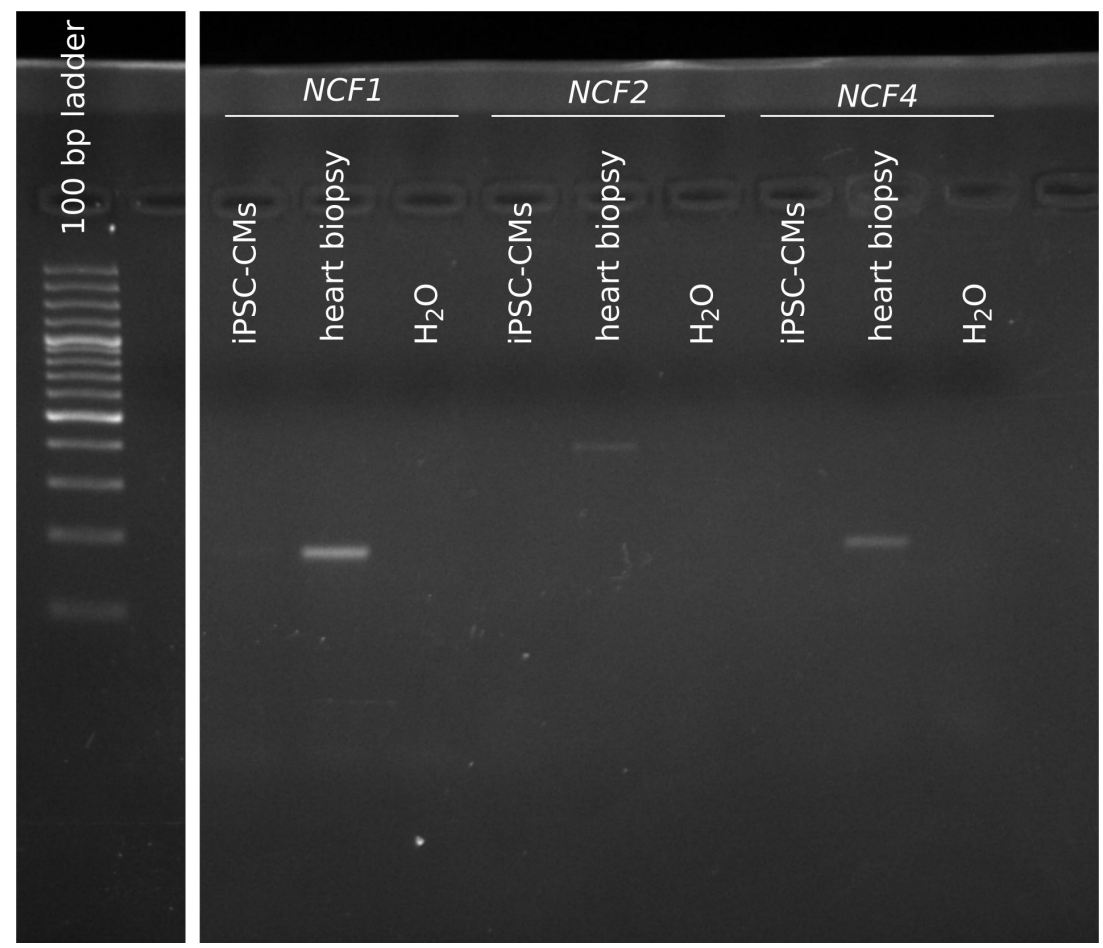

Figure 35: Verification of primers for amplification of NCF1, NCF2 and NCF4. Expression of NCF1, NCF2 and NCF4 was detected in a heart biopsy of a dilated cardiomyopathy patient but not in iPSCCMs.

Table 5: Mean CT-Values of qRT-PCR analyses.

\begin{tabular}{|l|l|}
\hline Gene & CT-value \\
\hline CamKII & 24 \\
\hline$c T N T$ & 17.94 \\
\hline CYBA & 30.41 \\
\hline GAPDH & 18.89 \\
\hline HPRT & 27.35 \\
\hline NCX & 21.33 \\
\hline NOX2 & 31.37 \\
\hline NOX4 & 29.85 \\
\hline PLN & 20.42 \\
\hline$R A C 1$ & 22.77 \\
\hline$R A C 2$ & 32.85 \\
\hline$R Y R 2$ & 25.32 \\
\hline SERCA & 21.9 \\
\hline
\end{tabular}




\begin{tabular}{|l|l|}
\hline Gene & CT-value \\
\hline$\alpha$-actinin & 17.9 \\
\hline$\alpha$-MHC & 23.95 \\
\hline$\beta$-MHC & 19.22 \\
\hline
\end{tabular}

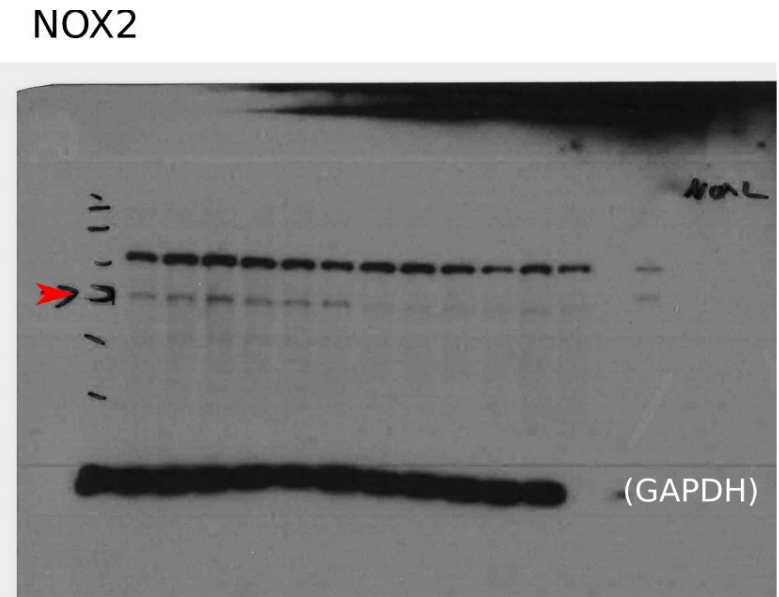

p40phox

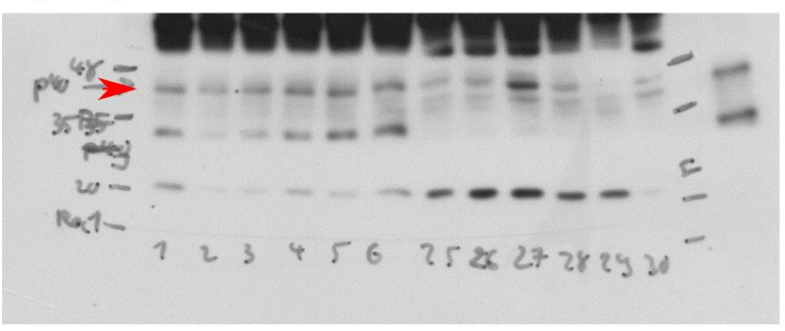

NOX4

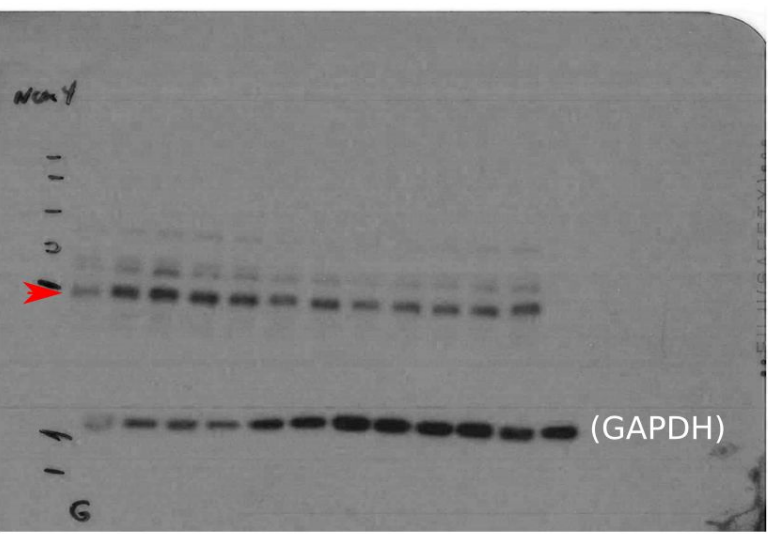

RAC1

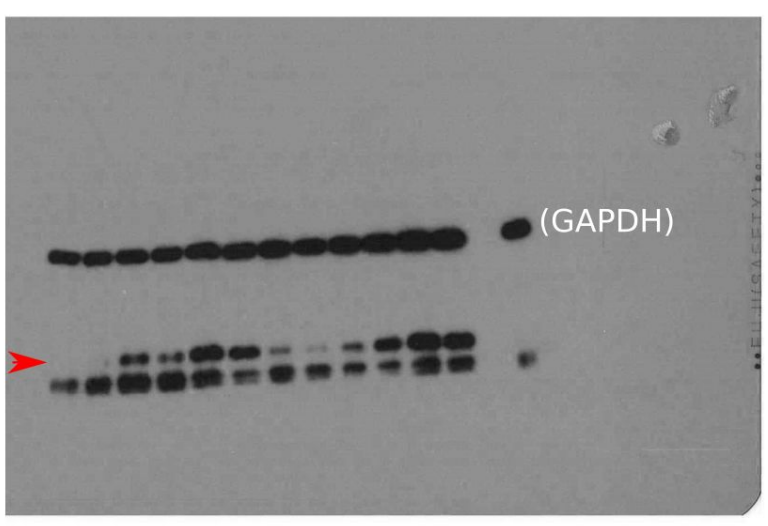

Figure 36: Original western blots of NADPH oxidase subunits. Bands used for quantification are marked with a red arrowhead. GAPDH was stained additionally on every membrane. 


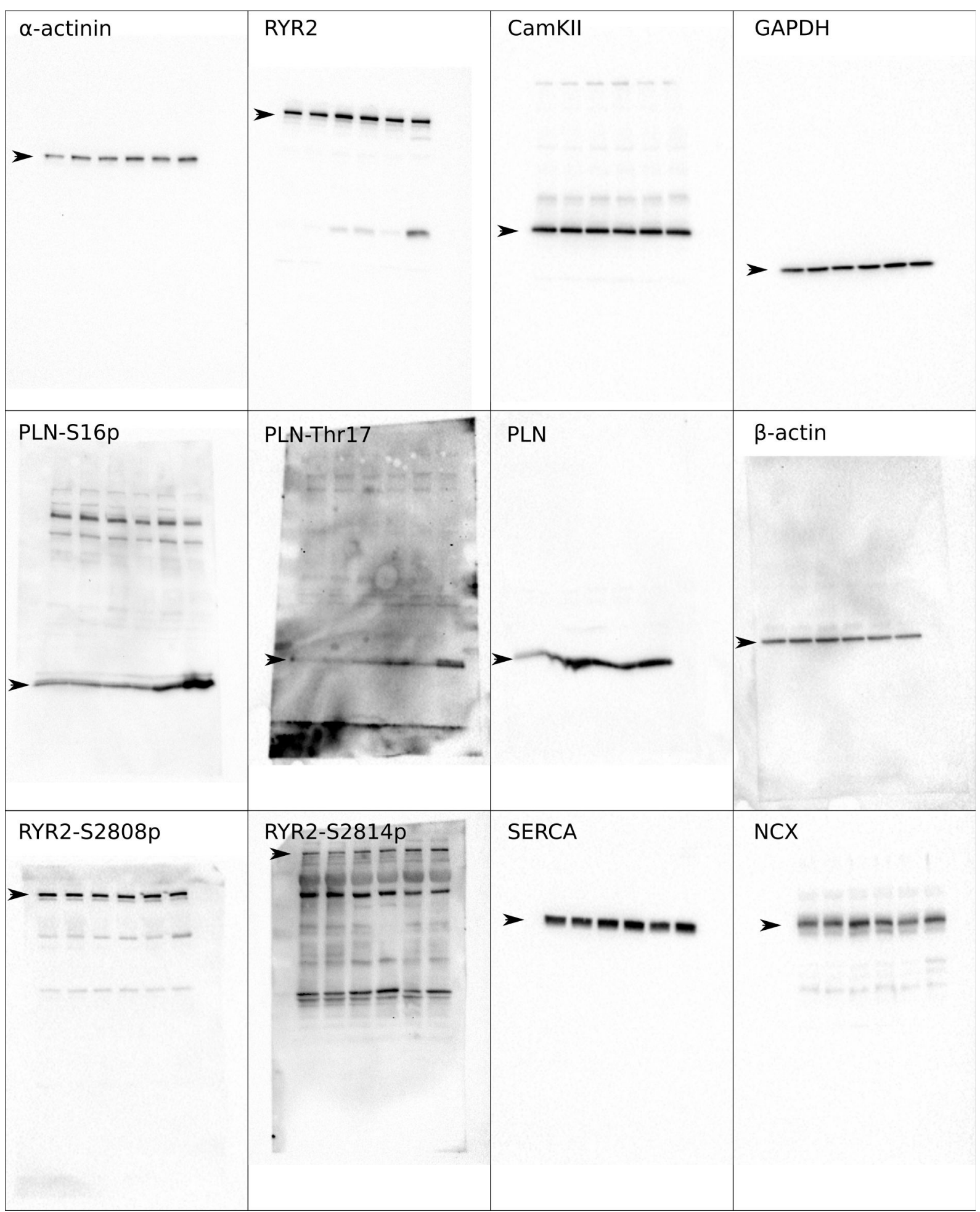

Figure 37: Original western blots of $\mathrm{Ca}^{2+}$ handling proteins, $\alpha$-actinin and reference proteins. Bands used for quantification are marked with an arrowhead. 


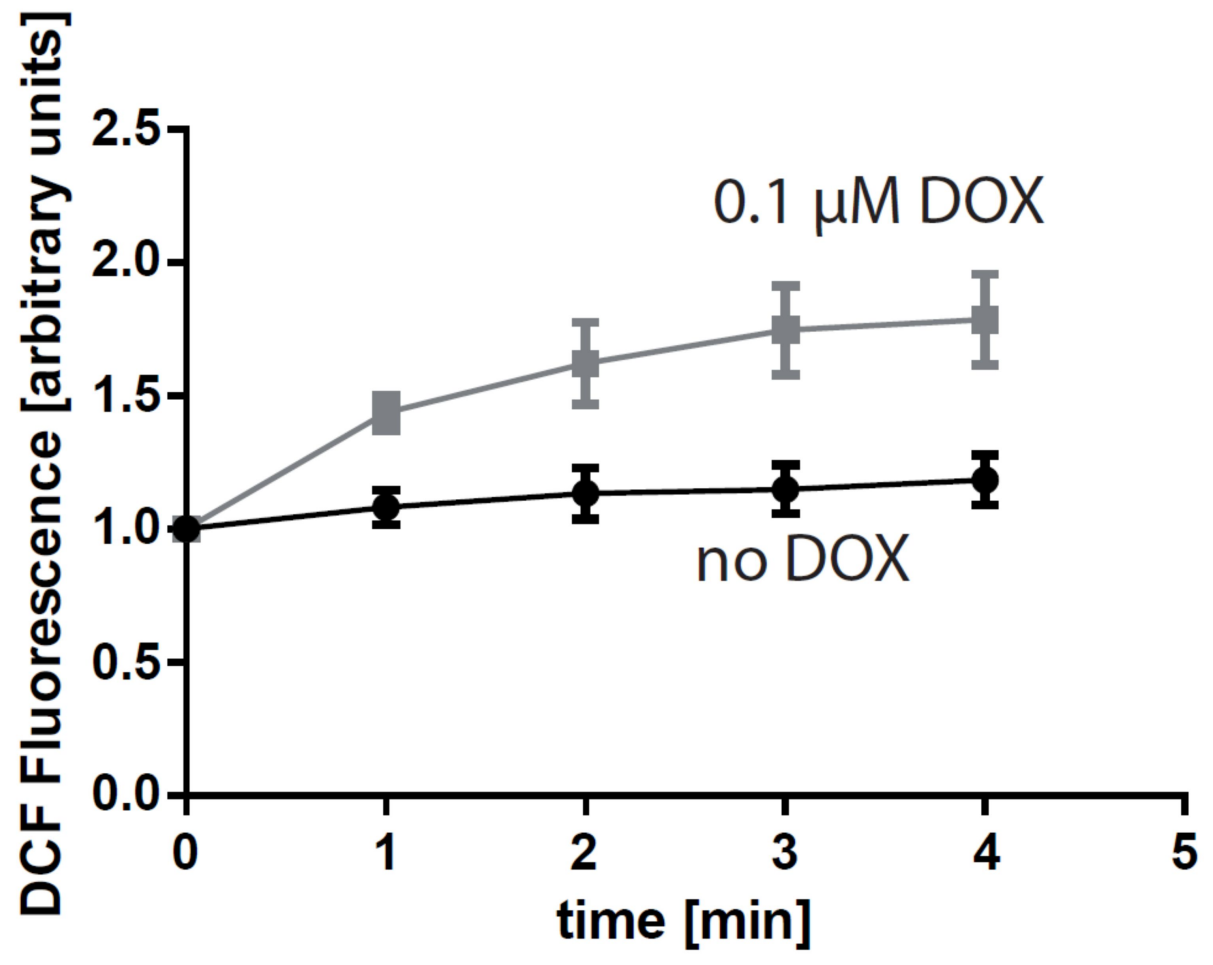

Figure 38: $\mathrm{H}_{2}$ DCF-DA assay trial in iPSC-CMs. $\mathrm{H}_{2}$ DCF-loaded iPSC-CM from a healthy donor were observed with confocal laser scanning microscopy in the frames can mode. The mean fluorescence intensity was normalized to the base value. Sample number: 2 . 


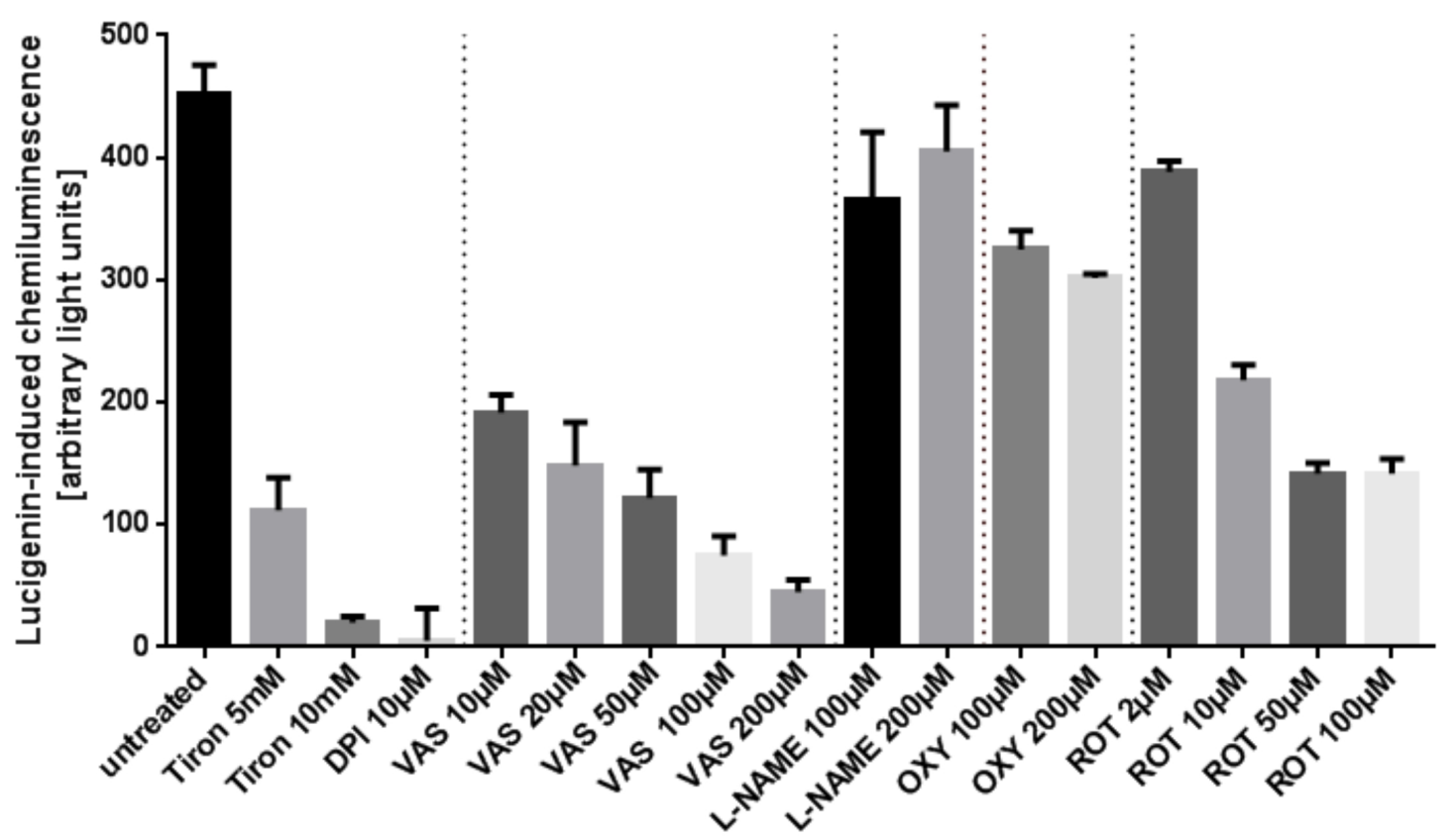

Figure 39: Lucigenin-enhanced chemiluminescence assay trial in iPSC-CMs. Homogenates of iPSC$\mathrm{CM}$ were treated with respective $\mathrm{O}_{2}^{-}$scavengers or enzyme inhibitors and $5 \mu \mathrm{M}$ lucigenin. Luminescence intensity was observed $10 \mathrm{~min}$ after addition of $300 \mu \mathrm{M} \mathrm{NADPH}$. Tiron is a $\cdot \mathrm{O}_{2}{ }^{-}$ scavenger. Diphenyleneiodonium (DPI) is a flavoprotein inhibitor. VAS2870 (VAS) is a NADPH oxidase inhibitor. NG-nitro-L-arginine methyl ester (L-NAME) is a nitric oxide synthase inhibitor. Oxipurinol $(\mathrm{OXY})$ is a xanthine oxidase inhibitor. Rotenone (ROT) is a mitochondrial electron chain inhibitor.

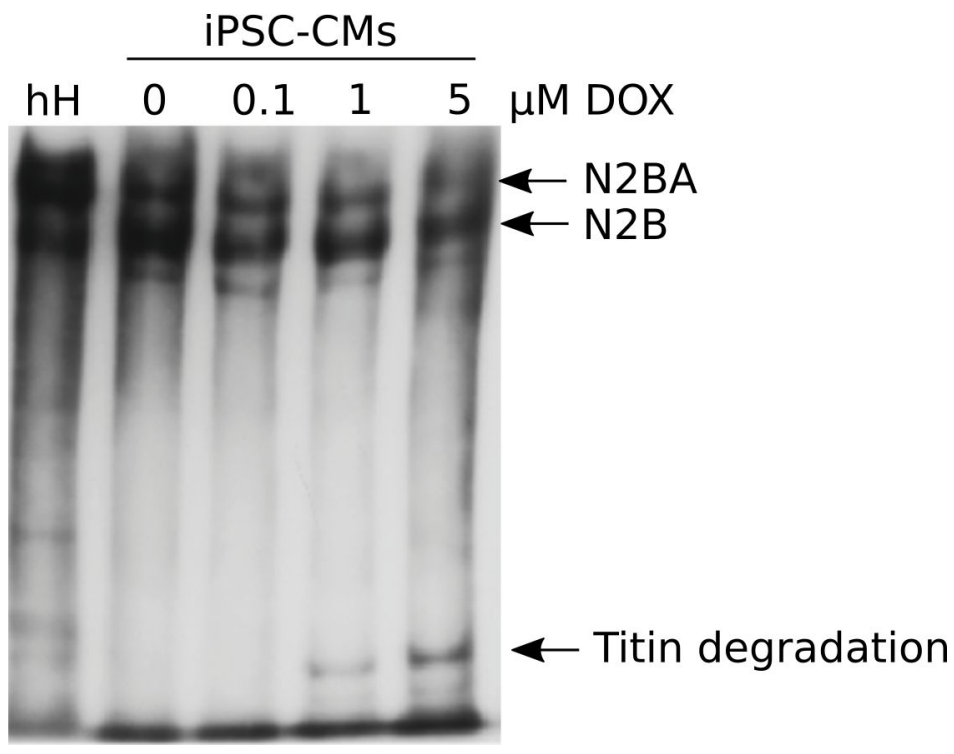

Figure 40: DOX-dependent titin degradation in iPSC-CMs. Western blot of a human heart biopsy sample $(\mathrm{hH})$ and DOX-treated iPSC-CMs from a healthy control donor. Titin was visualized with an antibody that is specific for the C-terminus of titin. N2BA and N2B: Titin isoforms. Data generated by Andrey Fomin. 


\section{Acknowledgments}

I would like to express my sincere gratitude to everyone who supported me during my doctoral studies over the last years.

First, I especially thank Dr. Katrin Streckfuß-Bömeke for giving me the opportunity to be a doctoral student in her laboratory, for providing me with such an interesting project and for excellent supervision. I also thank Prof. Dr. Gerd Hasenfuß for giving me the chance to work in the Department of Cardiology and Pneumology.

I am very grateful to Prof. Dr. Sigrid Hoyer-Fender for being the first reviewer of my thesis and making it possible for me to participate in the biological doctoral program. I learned scientific thinking during my bachelor's thesis in her research group and it was very special to me that she supervised me during my doctoral studies.

I thank my second reviewer Prof. Dr. Susanne Lutz for the constructive discussions and her kind advice during our annual committee meetings.

I also extend my thanks to Prof. Dr. Dörthe Katschinski, Prof. Dr. Ernst A. Wimmer and Prof. Dr. Rüdiger Behr for being part of my examination board despite their busy schedule.

I especially thank the people who donated skin biopsies for this work. They survived cancer and afterwards suffered from heart condition but still donated cells. I admire their courage and kindness.

Thanks go to Dr. Malte Tiburcy and Irina Eckhardt for the generation and analyses of EHMs. I also thank Dr. Andreas Petry for performing EPR experiments and NADPH oxidase western blots.

A big thank goes to the people who took the time to teach and brief me. I thank Dr. Lukas Cyganek for teaching me the measurement of intracellular calcium. I owe particular thanks to Dr. Eriona Heta for the Grx1-roGFP2 construct, the virus generation protocol, which worked like a charm, and her great help with the analyses. I thank Sabrina Becker for her foresightful briefing and teaching of flow cytometry. I also thank Thomas Borchert for writing the helpful makro for the analyses of calcium measurement data.

I thank Malina Seguin, Jérôme Janßen and Dirk Alexander Frick for their hard work during their theses or internships and for giving me the opportunity to learn to teach.

I owe particular thanks to all colleagues from the stem cell lab for their support. Special thanks go to Carmen Klopper and Sandra Georgi for their excellent technical assistance within the ACT project. 
Special appreciation goes to Andrey Fomin for his helpful scientific suggestions, his positive thinking and for reviewing my thesis.

I also thank Sabine Rebs, Jessica Spitalieri and Marco Singer for their helpful reviews of my thesis.

Many thanks to my best friend Marco Singer, who always supported me and cheered me up. My particular thanks go to my mother for her unconditional support and her understanding during busy times in the last years.

I would like to express my deepest gratitude to Jessica for her love, her understanding and her advice. 


\section{Curriculum vitae}

Name:

Address:

Birth:

E-mail:

Study

12.2013

02.2018

(expected)

$10.2011-$

11.2013

10.2010

09.2011

10.2007

09.2010
Luis Peter Haupt

Brunnengasse 2, 37077 Göttingen

April 23, 1987 in Braunschweig

luishaupt@gmx.de
PhD study program "Biology" at the Georg-August University

School of Science (GAUSS), Göttingen

Project: „Modeling anthracycline-induced cardiotoxicity with patientspecific iPSCs“

Institute: Department of Cardiology and Pneumology; University Medical Center Göttingen

Supervisor: Dr. Katrin Streckfuß-Bömeke

Master of Science "Developmental, Neural and Behavioral

Biology”, Georg-August-University Göttingen

Master's Thesis: „Generation and characterization of induced pluripotent stem cells from patients with CPVT"

Supervisor: PD Dr. Kaomei Guan-Schmidt

Grade: 1.1; with distinction

Master of Science Biosciences, University Osnabrück

Main focus: Developmental biology

Bachelor of Science Biology, Georg-August-University Göttingen Main focus: Molecular biosciences

Grade: 2.0

\section{Work experience}

$08.2012-10.2012$

$05.2013-10.2013$

\section{Education}

$09.1999-06.2006$
Graduate assistant at the stem cell laboratory, Department of Cardiology and Pneumology; University Medical Center Göttingen Project: Generation of transgenic ps-hiPSCs containing a cAMPbiosensor for FRET based analyses

Gymnasium Ricarda-Huch in Braunschweig

Degree: Abitur diploma (2.7) 


\section{Publications and presentations}

- „Generation of a KLF15 homozygous knockout human embryonic stem cell line using paired CRISPR/Cas9n, and human cardiomyocytes derivation"; C. Noack, L. Haupt, W.H. Zimmermann, K. Streckfuß-Bömeke, L. Zelarayán; August 2017; Stem Cell Research.

- „Assessing the influence of the genetic background on anthracycline-induced cardiotoxicity with human induced pluripotent stem cell-derived cardiomyocytes"; L. Haupt and K. Streckfuß-Bömeke; October 2016; EMJ Cardiology 4.1.

- "Assessing the influence of the genetic background on anthracycline-induced cardiotoxicity with human induced pluripotent stem cell-derived cardiomyocytes “; L. Haupt, et al.; poster presentation at the European Society of Cardiology Congress 2016 in Rome.

- „Modeling anthracycline-induced heart failure using hiPSC-derived cardiomyocytes“; L. Haupt, et al.; poster presentation at the annual meeting of the "Deutschen Gesellschaft für Kardiologie" (German Cardiac Society) 2015 in Mannheim.

\section{Memberships and Grands}

- European Society of Cardiology, professional member

- Deutsches Zentrum für Herz-Kreislaufforschung (German Center for Cardiovascular Disease), Young-DZHK member

- Traveling grand of the "Deutschen Gesellschaft für Kardiologie" (German Cardiac Society) for the annual meeting 2015 


\section{Affidavit}

Here I declare that my doctoral thesis entitled:

"Modeling anthracycline-induced cardiotoxicity with patient-specific iPSCs"

has been written independently with no other sources and aids than quoted.

Luis Peter Haupt

Göttingen, December 2017 